\title{
ASSESSING THE FRACTIONATED SPACECRAFT CONCEPT
}

$$
\text { by }
$$

\section{Charlotte Mathieu}

Licence, Economic and Social Sciences - Université P. Mendès France, 2003

Civilingenjör, Aeronautics and Astronautics - Kungliga Tekniska Högskolan, 2002

Diplôme d'Ingénieur, Aeronautics and Astronautics - Ecole Centrale Paris, 2002

Submitted to the Engineering Systems Division

in Partial Fulfillment of the Requirements for the Degree of

\section{MASTER OF SCIENCE IN TECHNOLOGY AND POLICY}

\author{
at the \\ Massachusetts Institute of Technology \\ June 2006
}

๑)2006 Massachusetts Institute of Technology.

All rights reserved.

Signature of Author

Technology and Policy Program, Engineering Systems Division

May 12, 2006

1

Certified by.

Assistant Professor of Aeronautics and Astronautics and Engineering Systems

Thesis Supervisor

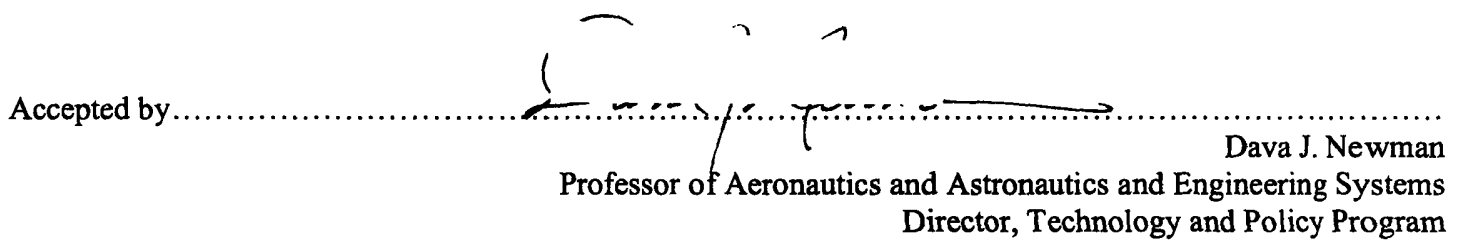

Director, Technology and Policy Program 


\title{
ASSESSING THE FRACTIONATED SPACECRAFT CONCEPT
}

\author{
by \\ Charlotte Mathieu \\ Submitted to the Engineering Systems Division on May 12, 2006 \\ in Partial Fulfillment of the Requirements for the Degree of \\ Master of Science in Technology and Policy
}

\begin{abstract}
In the traditional approach, spacecraft are tailored to each mission and are associated with high risks and costs and long cycles. Moreover, once launched, their flexibility remains limited. The concept of spacecraft fractionation could enable manufacturers and users to decrease these risks and costs and to increase space systems flexibility. Fractionation transforms the traditional monolithic spacecraft into a network of elements: a free-flying payload module is supported by free-flying modules that provide the payload with power, communications, etc. Thus modules could be maintained, exchanged, and reused once launched. Furthermore one could imagine developing a whole on-orbit infrastructure made of standardized modules that could support different payloads.
\end{abstract}

This thesis investigates under what conditions fractionated spacecraft could be worthwhile alternatives to traditional ones. The first part assesses different fractionated architectures and compares them with traditional ones in terms of utility and cost for a given mission and given level of performance. A framework based on Multi-Attribute Tradespace Exploration was used to analyze the impact of fractionation, first at spacecraft level for a single mission, and second, at infrastructure level, when the spacecraft is part of the infrastructure. The study demonstrates that if space assets flexibility is valued enough, customers would choose fractionated spacecraft over traditional ones.

The second part of the thesis examines the impact of spacecraft fractionation on the aerospace industry to understand why despite so many potential benefits, there are major barriers that prevent its implementation. Such an innovative concept could actually create a whole new paradigm in which today's protoflight approach would become a mass production approach, which would bring sweeping changes in today's space industry organization. This thesis explores policy options to increase the private sector's ability and motivation to implement fractionation and makes specific recommendations to enable the shift from today's paradigm to the fractionated spacecraft paradigm.

Thesis Supervisor:

Annalisa L. Weigel

Assistant Professor of Aeronautics and Astronautics and Engineering Systems 


\section{Acknowledgments}

I would like to thank my advisor, Dr. Annalisa L. Weigel, for providing me with an opportunity to work on such an exciting and challenging topic and for her guidance and supervision of this work.

I would also like to thank all the CASPARites - David Broniatowski, Greg Singleton, Mark Avnet, Karen Marais, Jenn Gustetic, and Thomas Coffe - for their precious feedback and support. I would also like to thank the SSPARC team, and especially Adam Ross for his valuable input on this research.

I would like to thank Dr. Owen Brown and Paul Eremenko for their insights and feedback.

Finally, I would like to thank the Defense Advanced Research Projects Agency (DARPA) for supporting the work that has made this thesis possible. 


\section{Executive Summary}

\section{A. Introduction}

1. The fractionated spacecraft concept

In today's approach, spacecraft are tailored to each mission. Their unique design and the protoflight approach used for their development and manufacturing lead to long cycles. As a result, designers tend to increase spacecraft lifetime which makes them grow larger and more complex. This trend leads to high costs and risks associated with each mission. Furthermore, traditional architectures have a limited ability to adapt to any of the changes that are likely to occur during the various phases of a spacecraft lifetime, especially once they are launched. The use of modular and standard components was a first step to reduce costs, risks, and cycles duration. However, the flexibility of these space systems remains limited. Reconfigurable spacecraft are now being developed to improve flexibility and decrease time constraints. Such new systems are based on standard modules that would be docked and undocked depending on the requirements. Taking modularity a step further means considering a spacecraft made of several smaller building blocks instead of a monolithic one. The technologies recently developed for distributed and cooperative space systems made conceivable this idea of fractionating spacecraft.

The concept of fractionated spacecraft transforms a traditional monolithic spacecraft into a network of elements: a free-flying payload module is supported by free-flying modules that provide the payload with communications, power, propulsion, etc. These supporting modules could be maintained, exchanged, and upgraded independently from the others and could be reused for different payloads. Thus fractionated spacecraft could offer much more flexibility, responsiveness, and survivability than the traditional monolithic spacecraft. To fully appreciate the potential benefits of this concept, the analysis must go further. The modules that contain these supporting functions could be standardized and reused for different missions. One could then imagine building a whole on-orbit infrastructure of such standardized modules that could support various payloads. Thus, today's bus platforms would no longer be designed and launched for a one-time use but would become a reusable infrastructure. This idea of an infrastructure of fractionated spacecraft revolutionizes the ideas of space-based capability and spacecraft lifetimes, creating a flexible, evolvable and scaleable system-of-systems infrastructure that would improve space utilization in a sustainable manner.

2. Assessment of the fractionated spacecraft concept

This thesis assesses the concept of fractionated spacecraft and examines under what conditions this innovative spacecraft architecture could be a worthwhile alternative to today's spacecraft architecture. Fractionation is assessed from two different and complementary perspectives.

The first part of this thesis assesses the concept of fractionated spacecraft from a customer standpoint. For specific missions, various fractionated architectures and an equivalent traditional architecture are compared in terms of cost and value delivered to customers. This architectural analysis demonstrates that if flexibility is valued enough with respect to other traditional performance parameters, customers would choose fractionated spacecraft over traditional ones. 
The second part of this thesis examines fractionation from the perspective of the industry. It investigates the new industrial paradigm created by fractionation and the dramatic changes to the space industry organization it could cause. It explains the reasons why the current industry players have neither the motivation nor the ability to implement fractionated spacecraft, so that despite many potential benefits, this concept is unlikely to be implemented without external intervention. The government, as such and as the first customer for fractionated spacecraft, is in the best position to enable the shift from today's paradigm to the new fractionated paradigm. This thesis explores policy options to make this shift possible and finally proposes a set of incentives to enable it.

\section{B. Architectural analysis}

The goal of the architectural analysis is to assess the concept of fractionated spacecraft from a customer perspective by quantifying the benefits and costs of fractionation for different missions. This study investigates different fractionated architectures and compares them to traditional ones in terms of cost and value delivered to potential customers. This comparison between fractionated and traditional spacecraft is done at two levels: first, at spacecraft level, when a single spacecraft is fractionated; second, at infrastructure level, when the spacecraft investigated is part of an overall infrastructure of fractionated spacecraft.

\section{Method used to assess the fractionated spacecraft}

\subsection{Multi-Attribute Tradespace Exploration framework}

The fractionated spacecraft concept is investigated using Multi-attribute Tradespace Exploration (MATE). This method takes a customer-centric approach as it assesses architectures in terms of the attributes valued by possible customers.

The different elements of the MATE framework are presented on Figure 1. First, a mission and the attributes valued by potential customers for such a mission are defined. Second, the architectures that could perform this mission are defined by a set of design parameters, which forms the design vector. The values of the design parameters that correspond to the architectures investigated define the tradespace of architectures. Third, the simulation model computes for each architecture the value of its attributes and its cost. In addition, the simulation model computes the utility of each architecture based on its attributes value. To be able to do so, utility interviews are used to determine for different users the single utility function associated with each attribute and the multiattribute utility function. This method finally provides figures of utility versus cost for the various spacecraft architectures. These results provide decision-makers with a comparison of the different fractionated architectures with the traditional one for a given mission, and enable them to identify the best architecture for the mission and to perform new trade-offs. The exploration of this tradespace allows also analysis of sensitivity to both design parameters and customer needs. 


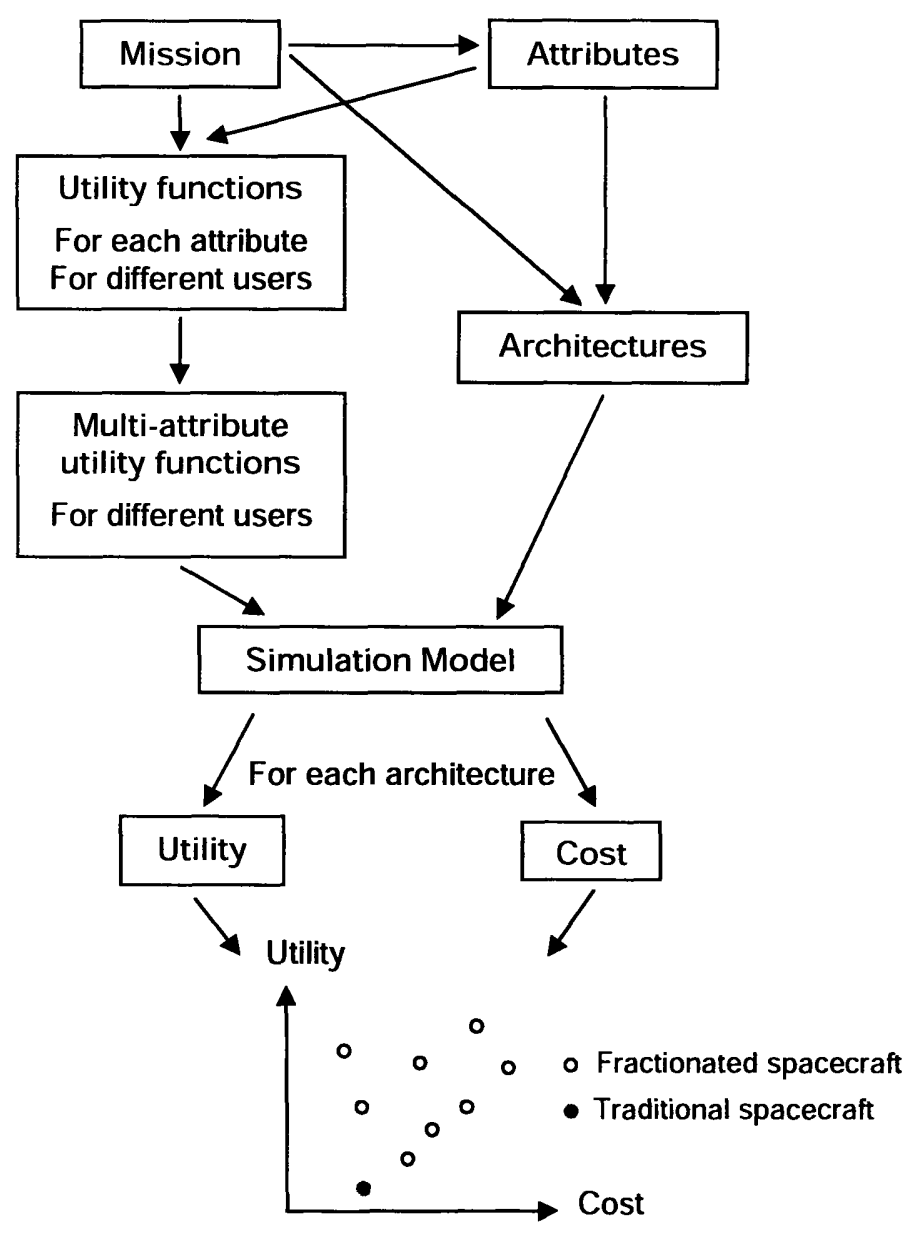

Figure 1: Architectures assessment method for a given mission

\subsection{Architectures assessment criteria}

The attributes chosen as evaluation criteria for this analysis are meant to reflect the value derived from space assets by any potential customer, allow a fair comparison of traditional and fractionated spacecraft in the same tradespace, and significantly vary between the traditional and fractionated spacecraft but also between the various fractionated spacecraft architectures. Thus, in this study, all the systems compared have the same level of performance and are built around the same payload. Therefore, what is measured is the variation in value delivered to the customer by these systems at a constant performance level.

Given that hypothesis of isoperformance, the attributes that are used to assess the architectures are non-traditional performance parameters that are likely to be valued in a dynamic, uncertain environment. Three uncertain changes that are taken into account in this study are: an unanticipated failure of the spacecraft, an increase in the demand for the capability delivered by the spacecraft, and the demand for a new capability. To each of them is associated the ability to adapt to this change in a cost-effective and in a timely manner. The six attributes used as architectures assessment criteria are therefore: 
maintainability, scalability, flexibility and the three corresponding forms of responsiveness. In addition, cost is taken into account as an independent variable.

\subsection{Architectures design parameters}

All the architectures investigated are defined by a two sets of design parameters. A first set of design parameters describes the fractionation of a single spacecraft, and additional set of parameters describes the infrastructure this spacecraft may be part of.

The three main design parameters at spacecraft level are: the spacecraft fractionation level, the number of infrastructure modules, and the power subsystem fractionation approach. The spacecraft fractionation level characterizes the degree of fractionation of the spacecraft architectures relative to each other. The $0 \%$ fractionation level corresponds to the traditional monolithic spacecraft. In the first step of the fractionation process, the possibility of wireless data transmission is considered, which leads to the complete fractionation of both the communications and the control and data handling subsystems. In the second step, the possibility of beaming power is considered, but the fractionation can be partial, on a continuum from 0 to $100 \%$ of fractionation, and two different approaches are taken into account: either both the power generation and storage function are fractionated at the same time, or the power generation is entirely fractionated in the power module and only the storage is distributed between the power module and the other modules. Finally, the possibility of collaborative separated positioning is considered, which would lead to the complete fractionation of both the attitude determination and control and the propulsion subsystems. The second dimension of fractionation at spacecraft level concerns the number of separate modules that form the infrastructure architecture. If the spacecraft investigated is assumed to be part of a larger infrastructure, there are additional parameters to take into account in the evaluation. First, a standardization parameter simply defined as the percentage of additional payload mass and power requirements the infrastructure modules could support, and second, the number of standardized modules produced to build the infrastructure.

\section{Results of the assessment}

General conclusions can be drawn from this architectural analysis at both spacecraft and infrastructure levels and for all missions. First, at a given performance level, utility significantly increases with fractionation, i.e. the overall utility of fractionated architectures is always higher than the utility of equivalent traditional architectures. However, utility does not always with the level of fractionation, i.e. from the least to the most fractionated architectures. Second, the initial cost of the fractionated architectures increases with fractionation and most of the time with the number of modules. At spacecraft level, the fractionated architectures are always more expensive than traditional ones, but at infrastructure level, depending on the infrastructure design parameters, some, or even all, fractionated architectures might be cheaper than the traditional architecture. Thus fractionated architectures can dominate traditional ones in terms of both utility and costs at a given performance level.

Then, there are conclusions that are specific to the mission. For communications missions, utility increases with fractionation and is the same for all the architectures with a same fractionation level. However costs increase with both fractionation and the 
number of modules, so that, at a given fractionation level, and therefore at a given utility level, the architecture with a single infrastructure module is the cheapest one. The architectures with only one infrastructure module are therefore the best architectures for these missions. For navigation missions, the first conclusion is that fractionated spacecraft may be a worthwhile alternative to traditional ones only if the infrastructure is developed. If only one spacecraft is fractionated, all the fractionated architectures are more expensive and have either a slightly higher or even a lower utility than an equivalent traditional spacecraft. The utility of the different architectures for this type of mission is mostly driven by maintainability attributes. Utility increases initially with fractionation but then decreases when the power subsystem gets fractionated. As a result, the architectures that have the communications, the control and data handling, and the power subsystems fractionated seem to be the best architectures for this type of mission. This architectural analysis finally proves that at a given performance level fractionated spacecraft may deliver more value to potential customers and even be cheaper than traditional ones. It demonstrates that if non-traditional attributes are valued enough, customers would choose fractionated spacecraft over traditional ones.

Finally, to complete this assessment of the fractionation concept from a customer standpoint, all the other benefits and the drawbacks associated with the development of an infrastructure of standardized fractionated modules are analyzed in terms of missions' costs, risks, performance and schedule.

\section{Industrial and Policy Analysis}

The second part of this thesis investigates the reasons why, despite so many potential benefits for customers, fractionated architectures are not implemented yet and is unlikely to be implemented without any intervention from outside the industry. A set of policies that could enable its implementation is proposed.

\section{A new industrial paradigm}

Fractionation and the development of an infrastructure of standardized modules create a whole new industrial paradigm in which the traditional protoflight approach take aspects of a mass production approach. Thus the traditional spacecraft customized and integral designs could become modular and standardized designs. Fractionation could make a modular manufacturing and Integration, Assembly and Test (IAT) possible and the development of an infrastructure of standardized modules could enable a massproduction approach. All the modules could be developed, manufactured, assembled, and tested in parallel, independently from each others. All the cycles could become much shorter. This change in the industrial paradigm would be associated with dramatic transformations in the space industry organization.

2. A new industrial organization

The implementation of this modular architecture and the development of an infrastructure of standardized modules could prompt sweeping changes to the space industrial base 
structure. The payload modules could still be provided by today's main spacecraft integrators but the infrastructure modules could be directly provided by today's subcontractors or even new companies. As a result, the distribution of profits and power in this sector might evolve. The industry could gain new dynamics, become much more competitive, and much less stable and concentrated. The shorter cycles could set a faster pace and new opportunities could be created more frequently.

This industrial analysis shows that the first barrier to the shift from today's paradigm to the fractionated spacecraft paradigm is the expected resistance to change from the large systems integrators that benefit from the status quo.

\section{Barriers to the implementation of fractionation}

This thesis demonstrates that, in addition to this resistance of some of the stakeholders, there are clearly too many financial, managerial and regulatory barriers that limit the various industry players' ability and motivation to implement fractionation. There are especially significant barriers associated with the development of the enabling technologies and with the development and management of the fractionated modules infrastructure.

\section{Policy recommendations}

There are several rationales for a government intervention that could enable the paradigm shift. First, the government would be the first customer of the infrastructure. Second, the government could correct some of the existing market failures that prevent the implementation of fractionation. Last but not least, the government could ensure the access of commercial customers to the infrastructure at marginal cost. Thus the government seems to be the most likely player that would intervene and enable the paradigm shift.

The various policy options that may lower these barriers are explored and the question of the initial market for fractionated spacecraft is addressed. The communications satellites market was identified as the first market to target; the infrastructure could be initially developed to support military communications missions and then its access may be opened to commercial communications payloads. Finally, a unique set of policies that would make the paradigm shift possible is proposed. This recommended chronological sequence of policies is the one that follows.

a. The government should commit to act as an "anchor tenant" for use of the infrastructure;

b. The government should invest in the enabling technologies;

c. The government should award R\&D contracts to different public-private partnerships to develop the enabling technologies, at least in the early stages;

d. Competition policy should be relaxed in order to allow a cooperative development of the fractionated spacecraft architecture;

e. The government should mandate the definition of the interfaces standards;

f. The government should create a centralized entity that would manage the infrastructure. 
The shift from today's traditional paradigm to the new fractionated spacecraft one might occur only if all of these policies are implemented. This list shows the challenge that this transition represents.

\section{Conclusions}

The first conclusion of the architectural analysis is that fractionated spacecraft could deliver more value to customers than traditional ones for a given mission and at a given level of performance. As demonstrated in the architectural analysis, the more fractionated the architecture is, the more expensive it is, but in terms of value delivered to potential customers the "best" fractionated architectures are different in the various fields of application (communications, navigation, etc.). For communications missions, the more fractionated the architecture is, the more utility it could deliver to customers, whereas for navigation missions utility tend to decrease with fractionation. These differences are linked to the technical characteristics of the spacecraft as well as to the difference in the needs of the users' communities. The utility interviews have shown that users in different fields of application show different interest in the various forms of flexibility. A second important conclusion of the architectural analysis is that, if the infrastructure is developed and for a same level of performance, some of the fractioned architectures may dominate traditional ones both in terms of cost and utility.

This thesis clearly demonstrates that fractionation could provide customers with many potential benefits, but it has also shown that this concept is unlikely to be implemented without external intervention, as the private sector has little incentive to implement it. This study shows that fractionation could create a whole new paradigm and presentes the new space industry organization that would correspond to this paradigm. It also demonstrates that the various industry players have neither the motivation nor the ability to make the shift from today's paradigm to the new fractionated spacecraft paradigm possible. The government, as such and as the first customer of the infrastructure, is in the best position to enable this transition. Thus, this thesis makes specific policy recommendations that address each of the barriers to the industry players' motivation and ability and that could make the paradigm shift possible. 


\section{Table of Contents}

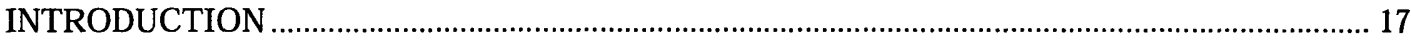

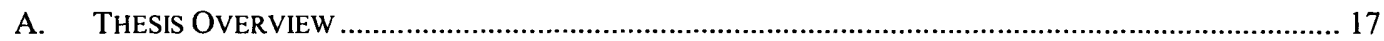

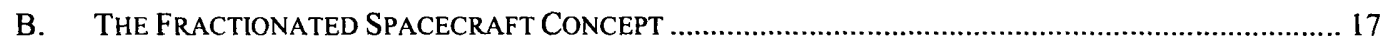

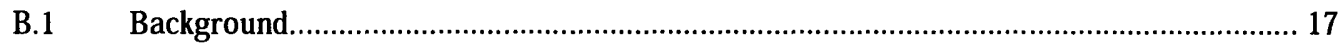

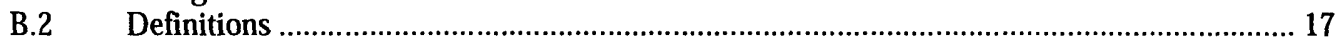

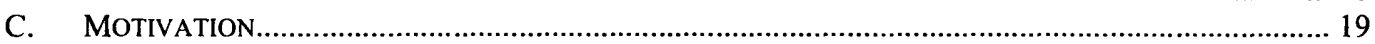

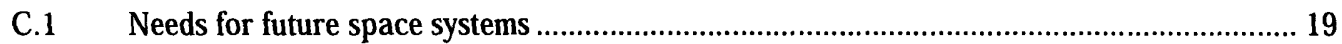

C.2 Potential benefits of spacecraft fractionation..................................................................... 19

C.3 Developing of an on-orbit infrastructure for a sustainable space utilization ........................ 19

C.4 The new fractionated spacecraft paradigm .................................................................. 20

D. RESEARCH GOALS AND APPROACH........................................................................................ 20

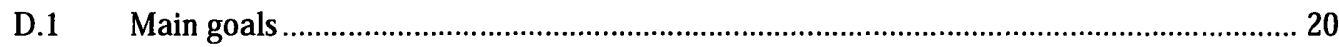

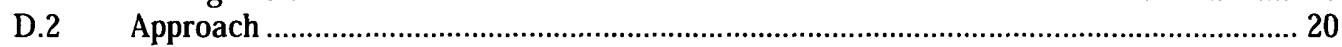

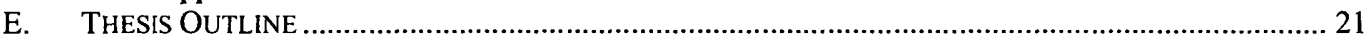

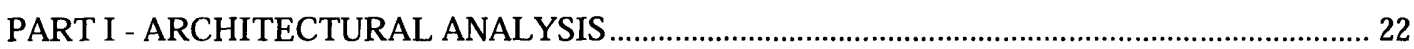

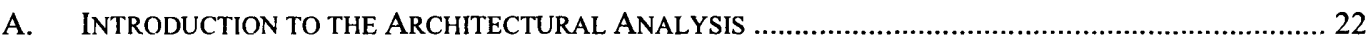

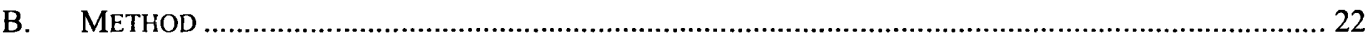

B.1 Multi-attribute tradespace exploration.................................................................... 22

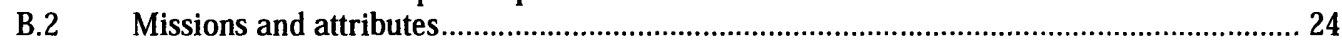

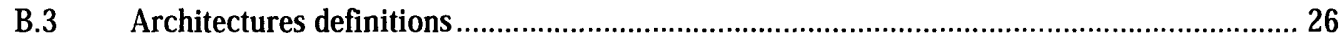

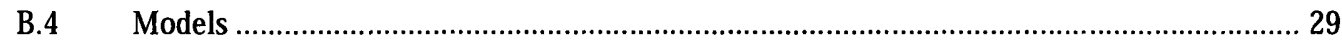

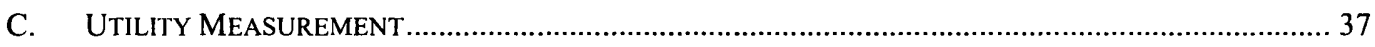

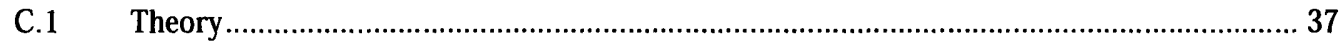

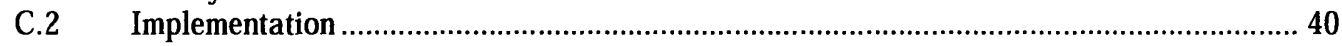

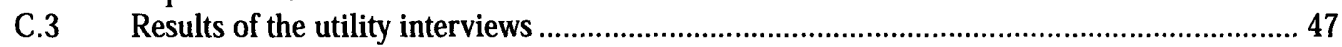

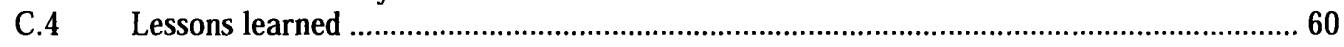

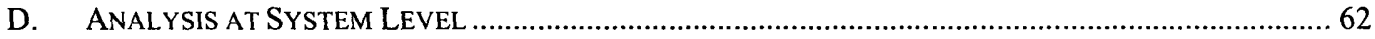

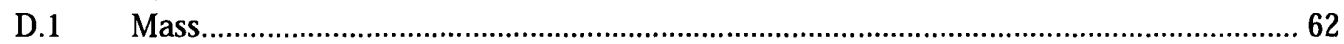

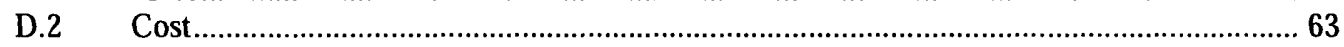

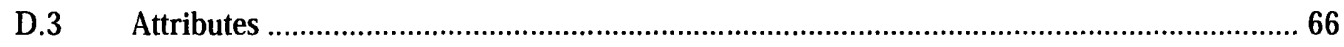

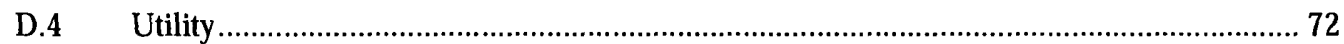

D.5 Multi-attribute utility ......................................................................................... 76

E. ANALYSIS AT INFRASTRUCTURE LEVEL ................................................................................... 79

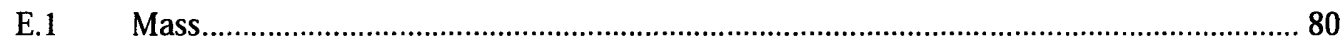

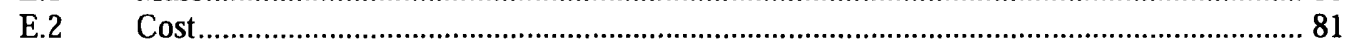

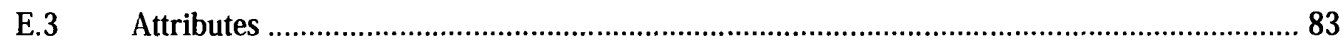

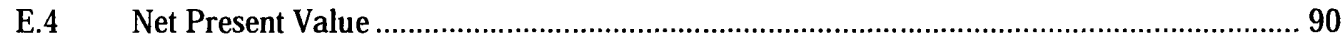

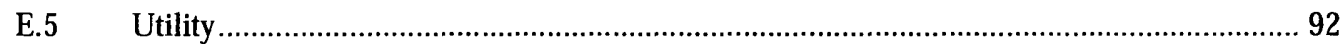

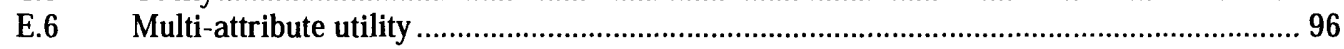

F. CONCLUSIONS OF THE ARCHITECTURAL ANALYSIS ............................................................ 105

F.1 Conclusions of the analysis of fractionated architectures in terms of cost and utility ........ 105

F.2 Other benefits and drawbacks of spacecraft fractionation ................................................. 106

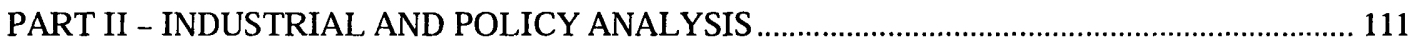

A. INTRODUCTION TO THE INDUSTRIAL AND POLICY ANALYSIS...................................................... 111

B. A New Fractionated Spacerraft Paradigm ……….................................................... 111

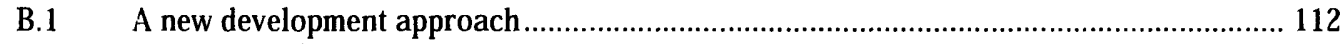

B.2 A new manufacturing and IAT approach................................................................ 112

B.3 Synthesis of both paradigms ................................................................................... 112

C. IMPACT OF SPACECRAFT FRACTIONATION ON THE SPACE INDUSTRY ..................................... 114

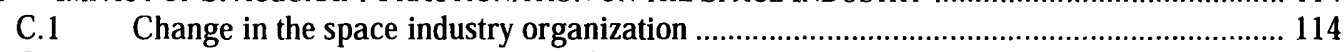

C.2 Consequences of fractionation on the space industry players ....................................... 120 
D. BARRIERS TO SPACECRAFT FRACTIONATION IMPLEMENTATION.......................................... 123

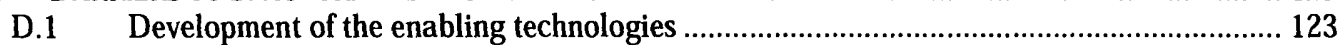

D.2 Development and management of the infrastructure .................................................. 124

E. POLICY RECOMMENDATIONS TO ENABLE THE PARADIGM SHIFT ............................................. 125

E.1 Ability and motivation to transition of the various players......................................... 126

E.2 Rationale for government intervention ............................................................ 127

E.3 Market(s) for which an infrastructure could be developed.......................................... 127

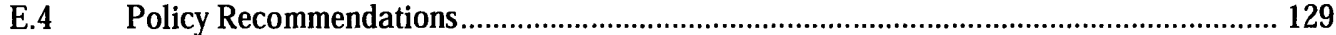

E.5 Impact of these recommended policies on the players' motivation and ability .................. 133

F. CONCLUSIONS OF THE INDUSTRIAL AND POLICY ANALYSIS ................................................. 134

F.1 Main conclusions of the industrial analysis......................................................... 134

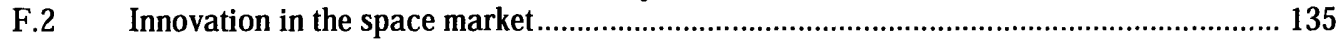

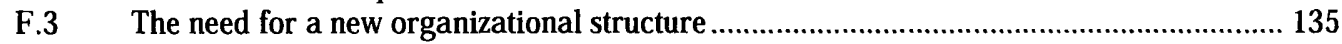

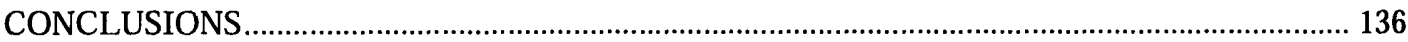

A. MAIN CONCLUSIONS OF THE ASSESSMENT OF THE FRACTIONATED SPACECRAFT CONCEPT ........... 136

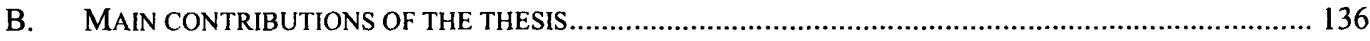

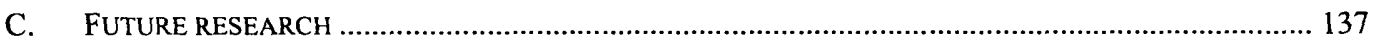

C.1 Further analysis of the fractionated architectures ..................................................... 137

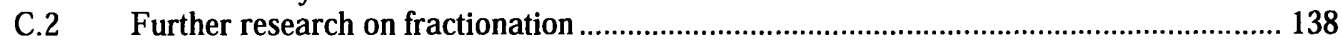

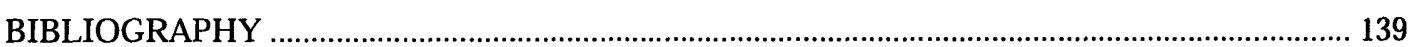

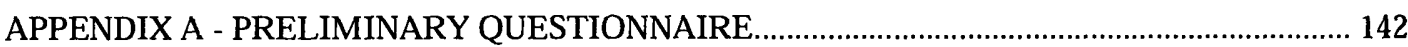




\section{List of Figures}

Figure 1: Traditional and fractionated spacecraft ........................................................................... 18

Figure 2: Architectures assessment method for a given mission ...................................................... 23

Figure 3: Framework to define the different kinds of "flexibility" .................................................... 25

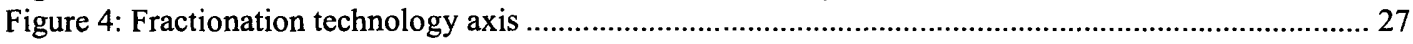

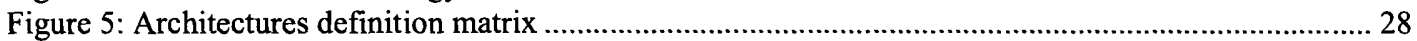

Figure 6: Main elements of the simulation model ............................................................................ 29

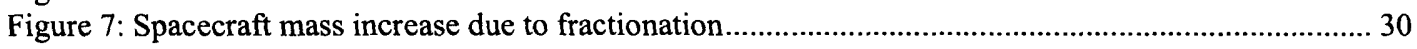

Figure 8: Format of the lotteries used for measuring single-attribute functions..................................... 38

Figure 9: Utility versus cost to maintain for different failure scenarios .............................................. 42

Figure 10: Utility versus upgrade costs for different scenarios .................................................... 42

Figure 11: Utility versus upgrade cost for different scenarios.......................................................... 43

Figure 12: Example of single-attribute utility question as presented to the interviewee .......................... 44

Figure 13: Format of the questions used to measure the scaling factor of the maintainability attribute....... 46

Figure 14: Utility versus satellite cost to get $50 \%$ additional power ................................................... 50

Figure 15: Utility versus time necessary to get $50 \%$ additional power ................................................. 51

Figure 16: Utility versus satellite cost to get anti-jam capability .................................................. 51

Figure 17: Utility versus time necessary to get anti-jam capability ................................................... 52

Figure 18: Utility versus cost to maintain the satellite in case of complete failure after 1 year ................. 56

Figure 19: Utility versus time to maintain the satellite in case of complete failure after 1 year.................... 56

Figure 20: Utility versus cost to get coverage of both poles............................................................... 57

Figure 21: Utility versus time to get coverage of both poles ............................................................. 57

Figure 22: Utility versus cost to get navigation inside buildings................................................... 58

Figure 23: Utility versus time to get navigation inside buildings ................................................... 58

Figure 25: Impact of fractionation on the communications satellite initial cost for different architectures . 64

Figure 26: Impact of fractionation on the initial cost of the supporting functions for different architectures

Figure 27: Impact of fractionation on the navigation satellite initial cost for different architectures .......... 65

Figure 28: Cost of the communications module exchange relative to the traditional spacecraft cost

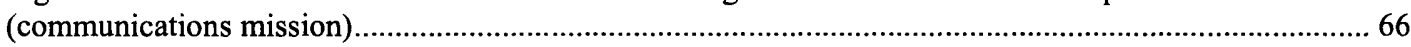

Figure 29: Cost of the power module exchange relative to the traditional spacecraft cost (communications mission)

Figure 30: Cost of the additional payload module relative to the scaled-up traditional spacecraft cost for different percentages of performance increase $p$ (communications mission) .......................................... 68 Figure 31: Cost of the new payload module relative to the traditional spacecraft cost (communications mission)

Figure 32: Time necessary to exchange the power module relative to the traditional spacecraft

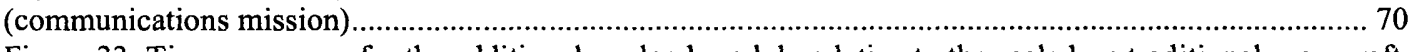

Figure 33: Time necessary for the additional payload module relative to the scaled-up traditional spacecraft for different percentages of performance increase $p$ (communications mission) ...................................... 71 Figure 34: Time necessary for a new payload module relative to the traditional spacecraft (communications

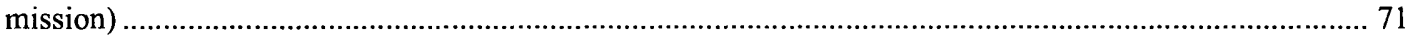

Figure 35: Utility associated with maintainability (navigation mission) ............................................... 72

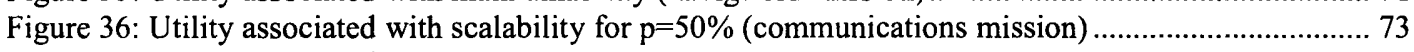

Figure 37: Utility associated with flexibility (communications mission) ............................................. 74

Figure 38: Utility associated with responsiveness (navigation mission) ............................................... 74

Figure 39: Utility associated with responsiveness for $\mathrm{p}=50 \%$ (communications mission) ........................ 75

Figure 40: Utility associated with responsiveness (communications mission) ........................................ 75

Figure 41: Utility versus fractionation level for a communications mission ......................................... 76

Figure 42: Utility versus initial cost for a communications mission .............................................. 77

Figure 43: Utility versus fractionation level for a navigation mission ................................................. 78

Figure 44: Utility versus initial cost for a navigation mission ....................................................... 79

Figure 45: Mass penalty due to fractionation for different fractionated architectures ............................... 80

Figure 46: Impact of fractionation on the communications satellite initial cost for different architectures . 81 
Figure 47: Impact of the infrastructure on the cost penalty for $s=30 \%$

Figure 48: Impact of fractionation on the navigation satellite initial cost for different architectures........... 83

Figure 49: Cost of the communications module exchange relative to the traditional spacecraft cost (communications mission)

Figure 50: Cost of the power module exchange relative to the traditional spacecraft cost (communications mission)

Figure 51: Cost of the additional payload module relative to the scaled-up traditional spacecraft cost for different percentages of performance increase $p$ (communications mission)....

Figure 52: Cost of the new payload module relative to the traditional spacecraft cost (communications mission)

Figure 53: Time necessary to exchange the power module relative to the traditional spacecraft

(communications mission).

Figure 54: Time necessary to get the additional payload module relative to the scaled-up traditional spacecraft for different percentages of performance increase $p$ (communications mission)

Figure 55: Time necessary to get a new payload module relative to the traditional spacecraft (communications mission).

Figure 56: Value at risk for a $95 \%$ confidence level and maximum net present values for different architectures in evaluating maintainability.

Figure 57: Notional net present value cumulative probability distribution for different architectures in evaluating scalability and flexibility

Figure 58: Utility associated with maintainability (navigation mission).

Figure 59: Utility associated with scalability for $\mathrm{p}=50 \%$ and $\mathrm{s}=30 \%$ (communications mission) .............. 93

Figure 60: Utility associated with flexibility (communications mission) ............................................... 94

Figure 61: Utility associated with responsiveness (navigation mission) .................................................. 95

Figure 62: Utility associated with responsiveness for $\mathrm{p}=50 \%$ and $\mathrm{s}=30 \%$ (communications mission)....... 95

Figure 63: Utility associated with responsiveness (communications mission)...................................... 96

Figure 64: Utility versus fractionation level for different standardization parameter s ............................ 97

Figure 65: Utility versus initial cost for $\mathrm{s}=30 \%$ and 25 units produced ............................................. 98

Figure 66: Utility versus initial cost for $\mathrm{s}=30 \%$ and 100 units produced ......................................... 98

Figure 67: Utility versus initial cost for $\mathrm{s}=0 \%$ and 100 units produced …............................................ 99

Figure 68: Utility versus fractionation level for a navigation mission for $s=30 \%$ and 100 units .............. 100

Figure 69: Utility versus initial cost for $\mathrm{s}=30 \%$ and 25 units produced .......................................... 102

Figure 70: Utility versus initial cost for $\mathrm{s}=30 \%$ and 25 units produced (zoom in) .............................. 102

Figure 71: Utility versus initial cost for $\mathrm{s}=30 \%$ and 100 units produced ............................................ 103

Figure 72: Utility versus initial cost for $\mathrm{s}=30 \%$ and 100 units produced (zoom in) ............................ 103

Figure 73: Utility versus initial cost for $\mathrm{s}=0 \%$ and 100 units produced .......................................... 104

Figure 74: Utility versus initial cost for $\mathrm{s}=0 \%$ and 100 units produced (zoom in) ............................... 104

Figure 75: Average NASA Small Spacecraft Mission Cost Breakdown (Sarsfield, 1998) ...................... 107

Figure 76: General pattern of invasion process ........................................................................... 110

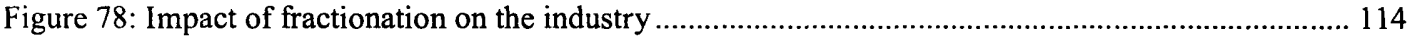

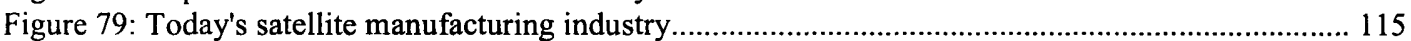

Figure 80: The suppliers of the different fractionated spacecraft modules........................................... 116

Figure 81: Industrial organization for the payload module ....................................................... 117

Figure 82: Industrial organization for the standardized infrastructure modules .................................. 118

Figure 83: Industrial organization in the new fractionated spacecraft paradigm.................................. 119

Figure 84: The motivation/ability framework .............................................................................. 126

Figure 85: The motivation and ability to implement fractionation of the current industry players ........... 127

Figure 86: Active US and multinational satellites (Nov. 2005)...................................................... 128

Figure 87: Public-private relationship on emerging technology S-curve (Chan, 2003) ........................ 130

Figure 88: Impact of the recommended policies on the players' ability and motivation to implement fractionation. 


\section{List of Tables}

Table 1: Definition of the attributes 26

Table 2: Design parameters at both levels

Table 3: The general scenarios associated with the attributes

Table 4: Scenarios defined by the communications expert

Table 5: Attributes scaling factors

Table 6: Attributes ranking based on the scaling factors

Table 7: Scenarios defined for each attribute by the navigation expert___ 55

Table 8: Attributes scaling factors

Table 9: Attributes ranking based on the scaling factors 


\section{Introduction}

\section{A. Thesis Overview}

This thesis assesses the concept of fractionated spacecraft and examines under what conditions this innovative spacecraft architecture could be a worthwhile alternative to today's spacecraft architecture. First, this thesis evaluates the concept of fractionated spacecraft from a customer perspective and demonstrates that if flexibility is valued enough with respect to other traditional performance parameters, customers would choose fractionated spacecraft over traditional ones. Second, this thesis analyzes the barriers to fractionated spacecraft implementation and proposes a strategy that could enable it.

\section{B. The Fractionated Spacecraft Concept}

\section{B.1 Background}

Traditional spacecraft are designed for one-time use. They are typically monolithic and have a tailored design. Because of the long development and manufacturing times, designers tend to increase the spacecraft lifetime and as a result spacecraft tend to grow larger and more complex. This tendency creates large costs and risks associated with a single mission and prevents use of most advanced technologies and reuse of launched elements. Moreover, these traditional architectures have a major drawback in that they limit the possible adaptations of spacecraft to the likely changes in their requirements or environment during their life cycle. The use of modular and standard components was a first step to reduce costs, risks, and development, manufacturing, and testing times. Reconfigurable spacecraft are now being developed to improve flexibility and decrease time constraints. Such new systems are based on standard modules that would be docked and undocked depending on the requirements. Taking modularity a step further means considering a spacecraft made of several smaller building blocks instead of a monolithic one. The technologies recently developed for distributed and cooperative space systems made conceivable this idea of fractionating spacecraft.

\section{B.2 Definitions}

The concept of fractionated spacecraft transforms a traditional monolithic spacecraft into a network of elements: a free-flying payload is supported by free-flying modules forming an on-orbit infrastructure.

\section{B.2.1 Homogeneous versus heterogeneous fractionation}

The concept of spacecraft fractionation transforms a large monolithic spacecraft into smaller modules. This division can be realized in two different ways: homogeneous and heterogeneous fractionation. Whereas homogenous spacecraft fractionation has been 
extensively studied, heterogeneous fractionation has only been recently introduced. The originality of this study is that it investigates this new concept.

Homogeneous fractionation replaces a large spacecraft with a cluster of smaller identical spacecraft. Those smaller spacecraft are simply scaled-down replicas of the initial spacecraft working in a collaborative manner to achieve an equivalent level of performance. Each of the smaller spacecraft is self-sufficient, can function independently from the others, and is designed in much the same way as traditional spacecraft.

On the other hand, heterogeneous fractionation divides the spacecraft into its functional elements. The payload and its supporting subsystems are implemented in different freeflying modules which all form a single system, as opposed to the system-of-systems resulting from the homogeneous fractionation. Heterogeneous fractionation is now conceivable given the technologies being developed for distributed space systems.

\section{B.2.2 The fractionated spacecraft concept}

The heterogeneously fractionated spacecraft is presented in Figure 1 along with the traditional spacecraft. In today's traditional approach, a spacecraft is built around a payload (slight shaded block), which is supported by various subsystems such as the power, communications, or propulsion subsystems (white blocks). An equivalent fractionated spacecraft consists of the same components but physically separated into a payload module and one or several infrastructure modules. The payload module contains a payload identical to the one in the traditional spacecraft (light shaded block) and the supporting functions that are not fractionated (medium shaded block). The infrastructure modules consist of the same supporting functions as in the traditional spacecraft (white blocks) and additional hardware (dark shaded blocks).

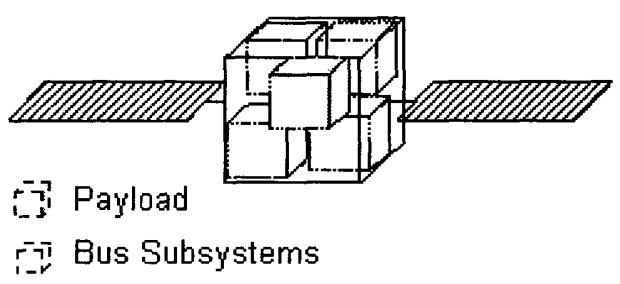

a) Traditional Spacecraft

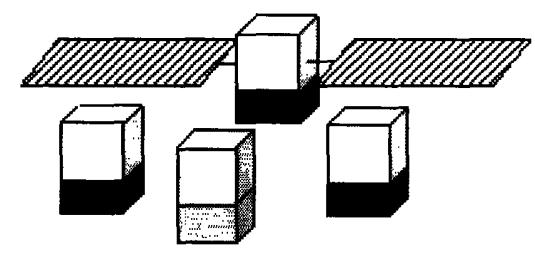

Payload Module

Infrastructure Modules

b) Fractionated Spacecraft

Figure 1: Traditional and fractionated spacecraft

The concept of fractionated spacecraft makes a clear distinction between the value-added payload and its supporting functions; supporting subsystem functions become reusable on-orbit infrastructure modules and are no longer designed and launched for each payload. 


\section{Motivation}

\section{C.1 Needs for future space systems}

Providers and users want their future space systems to have increased performance and to be more reliable and cheaper. In addition to improvements in these traditional performance features, they also call for more flexible space systems that could enable them to better manage the numerous uncertainties associated with space systems. Space systems actually often fail to meet new market conditions, to adapt to new applications, to incorporate the latest technologies and more generally to adapt to changes that may occur during their lifetime, in particular once they are launched (Nilchiani, 2005), which justifies the need for more flexibility. Space systems flexibility may include for instance the possibility of maintaining or servicing space assets once launched in case of unanticipated failure or degradation. To these desirable features for future space systems, military customers would add two others non-traditional ones due to the particularly uncertain and dynamic nature of their environment: operational responsiveness and survivability. Last but not least, a longer-term goal for all customers is more sustainable space utilization. Therefore, when designing future space systems all these attributes of performance and costs should be considered together and will obviously have to be traded against each other. This thesis demonstrates that the fractionated spacecraft could respond to these medium- and long-term needs and challenges in a unique way.

\section{C.2 Potential benefits of spacecraft fractionation}

The fractionated spacecraft concept turns the dominant integral monolithic spacecraft architecture into a highly modular one, and this modularity offers fractionated spacecraft many benefits over traditional ones. First, the different subsystems are no longer highly interconnected, therefore they can be developed, manufactured, integrated, and tested in parallel. This functional partitioning combined with the smaller size of the modules, lead to shorter design and build cycles, which means slower obsolescence as more recent technologies can be implemented on the system. Second, the modules can be launched separately which implies fewer spacecraft design constraints imposed by the launcher as well as less financial risk (Brown and al., 2005). Third, as the modules can be added, removed, or exchanged independently from the others, the fractionated spacecraft architecture offers much more flexibility, responsiveness, and survivability than the traditional spacecraft. It offers the possibility of on-orbit reconfigurability and significantly modifies the notion of spacecraft lifetime; modules can be developed with different lifetimes as a module's lifetime is no longer dependent on the other subsystems' lifetimes. Thus, fractionated spacecraft can provide customers with more flexibility, scalability, maintainability, and responsiveness than equivalent traditional spacecraft. But to fully appreciate the potential benefits of this concept, the analysis must go further.

\section{C.3 Developing of an on-orbit infrastructure for a sustainable space utilization}


As mentioned above, spacecraft fractionation makes a clear distinction between the value-added payload and its supporting functions. The modules that contain these supporting functions could be standardized and reused for different missions. One could then imagine building a whole on-orbit infrastructure of such standardized modules that could support various payloads. Thus, today's bus platforms would no longer be designed and launched for a one-time use but would become a reusable infrastructure. This idea of an infrastructure of fractionated spacecraft revolutionizes the ideas of space-based capability and spacecraft lifetimes, creating a flexible, evolvable and scaleable system-ofsystems infrastructure that would improve space utilization in a sustainable manner.

\section{C.4 The new fractionated spacecraft paradigm}

Fractionation creates a whole new paradigm in which today's protoflight approaches would become mass production approaches. The implementation of this modular architecture and the development of an infrastructure of standardized modules could prompt sweeping changes to the space industrial base structure. The industry would gain new dynamics, become much more competitive, and much less stable and concentrated. The shorter cycles would set a faster pace and new opportunities would be created more frequently. However, despite many potential benefits, there are still major barriers to the shift from today's paradigm to a new fractionated spacecraft paradigm. This study explores potential policy options to enable this transition.

\section{Research goals and approach}

\section{D.1 Main goals}

The concept of fractionated spacecraft has been only recently introduced and is still in an early stage of research. This thesis demonstrates the potential benefits of spacecraft fractionation and identifies the conditions under which fractionated spacecraft may be worthwhile alternatives to traditional ones.

\section{D.2 Approach}

The fractionated spacecraft concept is assessed from two different and complementary perspectives. The first part of this thesis assesses the concept of fractionated spacecraft from a customer standpoint by investigating different fractionated spacecraft architectures. The goal of this architectural analysis is to compare, under different assumptions, various fractionated architectures and an equivalent traditional architecture in terms of cost and value delivered to customers in order to identify the "best" architectures for specific missions. The framework developed for this analysis is based on Multi-Attribute Tradespace Exploration (MATE) and is applied to communications and navigation missions. This analysis is done at two levels: first at spacecraft level, only one spacecraft is fractionated, and then at infrastructure level when the spacecraft is part of the overall on-orbit infrastructure. The second part of this thesis examines fractionation from the perspective of the industry. It investigates the new industrial paradigm created by fractionation and the dramatic changes to the space industry organization it could 
cause. It explains the reasons why the current industry players have neither the motivation nor the ability to implement fractionated spacecraft, so that despite many potential benefits, this concept is unlikely to be implemented without external intervention. The government, as such and as the first customer for fractionated spacecraft, is in the best position to enable the shift from today's paradigm to the new fractionated paradigm. This thesis explores policy options to make this shift possible and finally proposes a set of incentives to enable it.

\section{E. Thesis Outline}

The first part of this thesis presents the architectural analysis. Chapter A introduces this analysis. Chapter B explains the general method used and the framework developed to assess different fractionated architectures and compare them with traditional ones for a given mission. Chapter $\mathrm{C}$ presents the results of the interviews of two potential customers, which were designed to capture their needs and preferences for different space systems attributes. Chapter D and E summarize the results of the architectural analysis, at spacecraft and infrastructure level respectively, for communications and navigation missions. Chapter $\mathrm{F}$ concludes this analysis and summarizes the potential benefits and drawbacks of fractionation from a customer perspective.

The second part of this thesis presents the industrial and policy analysis. Chapter A introduces this analysis. Chapter B investigates the new industrial paradigm created by fractionation. Chapter $\mathrm{C}$ analyzes the impact of fractionation on the different space industry players. Chapter D examines all the barriers to the fractionated spacecraft implementation. Chapter E explores different policy options to make the transition to the new fractionated spacecraft paradigm possible and makes specific recommendations. Chapter F concludes this industrial and policy analysis.

A last part of this thesis summarizes the conclusions of this assessment, the main contributions of this thesis and makes recommendations for future work. 


\section{Part I - Architectural Analysis}

\section{A. Introduction to the Architectural Analysis}

The goal of the architectural analysis is to assess the concept of fractionated spacecraft from a customer perspective by quantifying the benefits and costs of fractionation for different missions. To evaluate this new architectural concept, this study investigates different fractionated architectures and compares them to traditional ones in terms of cost and value delivered to potential customers. This comparison between fractionated and traditional spacecraft is done at two levels: first, at spacecraft level, when a single spacecraft is fractionated; second, at infrastructure level, when the spacecraft investigated is part of an overall infrastructure of fractionated spacecraft. This first part of the thesis demonstrates that, for certain missions and at a given performance level, customers would choose fractionated spacecraft over traditional ones.

The first chapter describes the method used to evaluate the concept of fractionated spacecraft. Second, the approach used to quantify the preferences of potential customers and the results obtained from two customers are presented. The third and fourth chapters illustrate typical intermediary and final results of the architectural analysis, respectively at spacecraft and at infrastructure levels. Finally, the main conclusions of this assessment are presented in the last chapter.

\section{B. Method}

\section{B.1 Multi-attribute tradespace exploration}

The fractionated spacecraft concept is investigated using Multi-attribute Tradespace Exploration (MATE) (Ross, 2003). This method takes a customer-centric approach as it assesses architectures in terms of the attributes valued by possible customers. The attributes taken into account can include traditional ones such as performance, as well as non-traditional ones such as flexibility, and each of them are evaluated independently from the others.

Other methods have been used to quantify the value of different system architectures in terms of non-traditional performance parameters (Christian and Olds, 2005) (Nilchiani and al., 2004). But the unique features of MATE allow to systematically investigate and compare architectures and to quantify the utility of these architectures for potential customers. Moreover, these architectures can be very different, as long as they can be described by a same set of parameters.

The different elements of the MATE framework are presented in Figure 2. First, a mission and the attributes valued by potential customers for such a mission are defined. Second, the architectures that could perform this mission are defined by a set of design parameters, which forms the design vector. The values of the design parameters that correspond to the architectures investigated define the tradespace of architectures. Third, the simulation model computes for each architecture the value of its attributes and its cost. In addition, the simulation model computes the utility of each architecture based on 
its attributes value. To be able to do so, utility interviews are used to determine for different users the single utility function associated with each attribute and the multiattribute utility function. This method finally provides figures of utility versus cost for the various spacecraft architectures. These results provide decision-makers with a comparison of the different fractionated architectures with the traditional one for a given mission, and enable them to identify the best architecture for the mission and to perform new trade-offs. The exploration of this tradespace allows also analysis of sensitivity to both design parameters and customer needs.

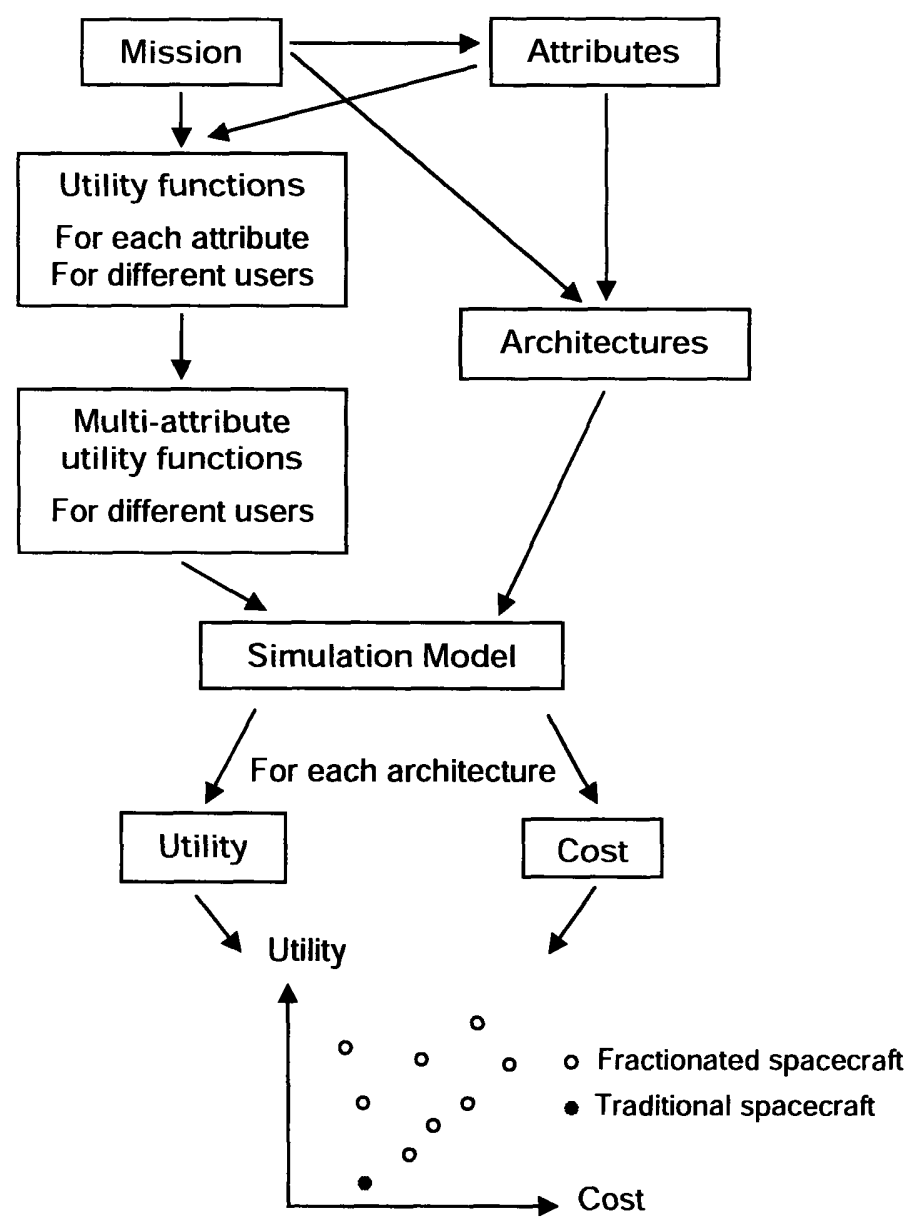

Figure 2: Architectures assessment method for a given mission

This method has mainly been used to assess different aerospace systems architectures, (McManus and al., 2004) (Spaulding, 2003), but some of its elements, for instance the use of multi-attribute utility functions, have been used in numerous others fields (Roth and al., 1994). 


\section{B.2 Missions and attributes}

\section{B.2.1 Missions}

In this study, the missions investigated include missions in the three main fields of application: communications, navigation, and remote sensing. Only communications and navigation missions are in this thesis fully investigated in terms of utility and cost, but the same framework can be applied to remote sensing applications.

\section{B.2.2 Architectures assessment criteria}

The attributes chosen as evaluation criteria for this analysis are meant to reflect the value derived from space assets by any potential customer, allow a fair comparison of traditional and fractionated spacecraft in the same trade-space, and significantly vary between the traditional and fractionated spacecraft but also between the various fractionated spacecraft architectures. Thus, in this study, all the systems compared have the same level of performance and are built around the same payload. Therefore, what is measured is the variation in value delivered to the customer by these systems at a constant performance level.

Given that hypothesis of isoperformance, the attributes considered are non-traditional performance parameters: maintainability, scalability, flexibility and the three corresponding forms of responsiveness. These six attributes represent non-traditional attributes likely to be valued in a dynamic, uncertain environment. In addition, cost is taken into account as an independent variable.

One of the contributions of this thesis is to use non-traditional performance parameters as attributes, as MATE has been used so far with attributes that are traditional performance parameters.

\section{B.2.3 Definition of the attributes}

The "flexibility" of a complex system can be understood in many different ways and this generic term often encompasses many different kinds of "ilities", from adaptability to scalability. It is crucial in this analysis to clearly define what is meant by those "ilities" before using any as an assessment criterion. A framework that was developed to better understand the differences and similarities between those "ilities" is presented below.

\section{B.2.3.1 A framework to analyze the non-traditional "ilities" attributes}

Many non-traditional "ilities" attributes can be broadly defined as "the ability of a system to adapt to uncertain internal or external changes affecting its functionality and performance, in a timely and cost-effective manner" (Nilchiani and Hastings, 2004).

There are different kinds of uncertainties and various ways for the system to adapt to those changes, so that any response to a change can be defined depending on:

$$
\begin{aligned}
& \Rightarrow \text { The nature of the change } \\
& \Rightarrow \text { The nature of the response } \\
& \Rightarrow \text { The time of response }
\end{aligned}
$$

Figure 3 graphically presents this framework.

There are at least two kinds of changes that can occur during a system lifetime: a change in requirements or a change in conditions. A change in requirements can be characterized as either: (a) a change in the functionality required, i.e. the system is required to perform 
a new function, or the same functional but in a different environment; or (b) a change in the level of performance required, i.e. the system is required to perform the same initial function but with an increased level of performance. A change in conditions may be either internal or external to the system (Nilchiani and Hastings, 2004). An internal change may correspond to a component failure or a system failure. An external change may correspond to a change in the environment surrounding the payload or at the interface between the system and its surrounding environment. In some cases, a change may result in changes of other kinds.

Each change and how the system adapts to each of them must then be analyzed sequentially.

The response to an initially unexpected change can be of two kinds: active or passive. The passive response can be understood as a tolerance of the system to change and is often linked to the concept of robustness. The possible active responses tend to be system-specific. Possible adaptations with a fractionated architecture consist of: reconfiguration, subtraction, addition, or exchange of elements.

The response of the system to a change can take place within different timeframes. This leads to different kinds of adaptations to the change depending on the importance given to the time factor. Three timeframes are defined in this framework: short-, medium- and long-terms. These timeframes are all relative and depend mainly on the system and user perspective.

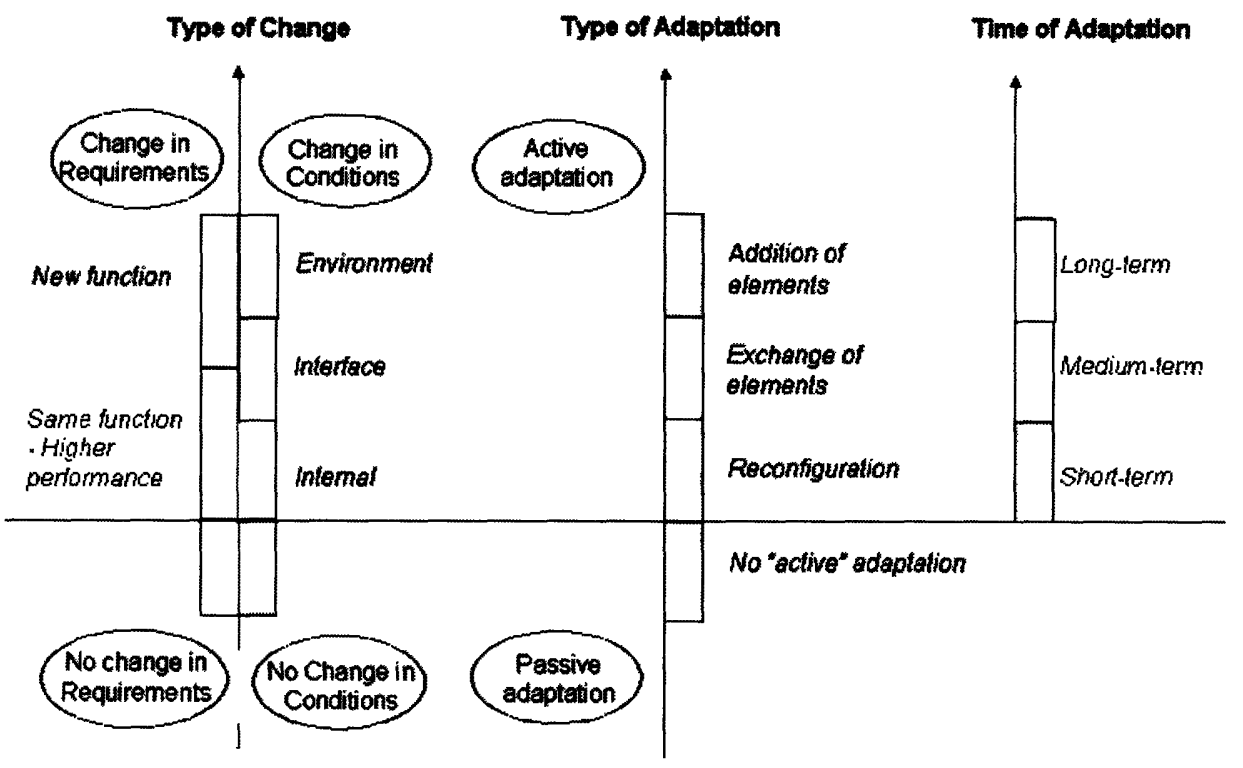

Figure 3: Framework to define the different kinds of "flexibility"

\section{B.2.3.2 Definition of the attributes}

Each attribute used in this analysis can be defined using this framework, as shown in Table 1.

- Maintainability can be defined as the "ability of a system to be kept in an appropriate operating condition" (Allen and al., 2003), which corresponds in the framework to a change in internal conditions leading to an exchange of element(s).

- Scalability can be defined as the "ability of a system to maintain its performance and function, and retain all its desired properties when its scale is increased greatly without 
having a corresponding increase in the system's complexity" (Allen and al., 2003). This corresponds in the framework to a change in requirements, namely an increased performance level.

- Flexibility can be defined as the "ability of the system to be modified to do jobs not originally included in the requirements definition" (Hastings and McManus, 2005). This would correspond in the framework to a change in requirements, namely a change in function, which would require an active response.

- Responsiveness can be defined as the ability to meet changing requirements or conditions quickly, which corresponds in the framework to a short- or medium-term adaptation to any change in requirements or conditions. Thus a form of responsiveness can be associated to each change in requirements and conditions, as can be seen in Table 1.

\begin{tabular}{|c|c|c|c|c|c|c|c|c|c|c|c|c|c|c|c|}
\hline & \multicolumn{3}{|c|}{$\begin{array}{c}\text { Change in } \\
\text { requirements }\end{array}$} & \multicolumn{4}{|c|}{$\begin{array}{l}\text { Change in } \\
\text { conditions }\end{array}$} & \multicolumn{5}{|c|}{ Type of response } & \multicolumn{3}{|c|}{$\begin{array}{c}\text { Time of } \\
\text { response }\end{array}$} \\
\hline & $\begin{array}{l}0 \\
\stackrel{0}{0} \\
\frac{0}{0} \\
\frac{5}{0} \\
0 \\
2\end{array}$ & 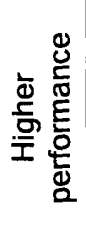 & 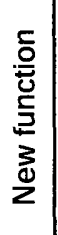 & 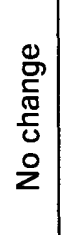 & 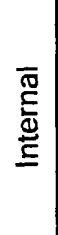 & 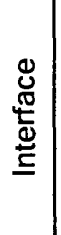 & 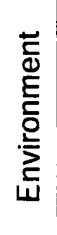 & 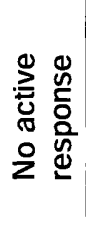 & 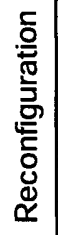 & 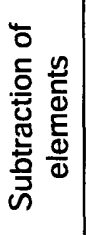 & 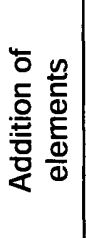 & 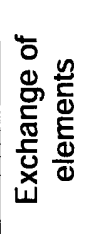 & 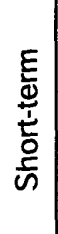 & 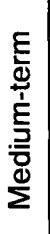 & \\
\hline Maintainability & $x$ & & & & $x$ & & & & & & & $x$ & $\bar{x}$ & $\mathrm{X}$ & $x$ \\
\hline Scalability & & $x$ & & $\mathrm{X}$ & & & & & $x$ & & $\mathrm{x}$ & $\bar{x}$ & $\bar{x}$ & $\bar{X}$ & $x$ \\
\hline "Flexibility" & & & $\mathrm{X}$ & $\mathrm{X}$ & & & & & $\mathrm{X}$ & $\mathrm{x}$ & $\mathrm{X}$ & $\mathrm{x}$ & $\mathbf{X}$ & $\mathrm{X}$ & $\mathrm{X}$ \\
\hline Responsiveness & $\mathrm{X}$ & $\mathrm{X}$ & $\mathrm{X}$ & $\mathrm{x}$ & $\mathrm{X}$ & $\mathrm{x}$ & $\mathrm{x}$ & & $\mathrm{x}$ & $\mathrm{x}$ & $\mathrm{X}$ & $x$ & $\mathrm{x}$ & $\mathrm{X}$ & \\
\hline $\begin{array}{c}\text { Responsiveness } \\
\text { (Maintainability) }\end{array}$ & $\mathrm{x}$ & & & & $x$ & & & & & & & $\mathrm{x}$ & $\mathrm{x}$ & $x$ & \\
\hline $\begin{array}{r}\text { Responsiveness } \\
\text { (Scalability) }\end{array}$ & & $x$ & & $\mathrm{x}$ & & & & & $\mathrm{x}$ & & $x$ & $x$ & $x$ & $\mathrm{x}$ & \\
\hline $\begin{array}{r}\text { Responsiveness } \\
\text { ("Flexibility") }\end{array}$ & & & $\mathrm{x}$ & $\mathrm{x}$ & & & & & $\mathrm{x}$ & $x$ & $\mathrm{x}$ & $\mathrm{x}$ & $\mathrm{x}$ & $\mathrm{x}$ & \\
\hline
\end{tabular}

Table 1: Definition of the attributes

\section{B.3 Architectures definitions}

a. Design parameters at system level

At the spacecraft level, the design parameters describing the architectures examined are:

- The subsystems fractionation level

- The technologies taken into account

- The number of infrastructure modules

- The subsystems contained in these modules

In a fractionated architecture, the supporting functions provided by the traditional spacecraft bus are distributed between the payload module and the infrastructure modules. A level of fractionation, from 0 to $100 \%$, can then be defined for each subsystem, representing the distribution of this function between the module dedicated to that function and the rest of the modules. The subsystems considered as potentially "fractionable" in this study are the communications, control and data handling, power, attitude determination and control, and propulsion subsystems.

A fractionation level can also be defined at the spacecraft level, and can characterize the degree of fractionation of the spacecraft architectures relative to each other. This 
spacecraft fractionation level has two main dimensions. The first is the supporting function distribution between the payload and the infrastructure, and the second is the number of physically separate infrastructure modules.

Distribution of the supporting functions between the payload and the infrastructure is linked to the technologies considered. Several subsystems are considered "fractionable", but some are more easily "fractionable" and it seems logical to fractionate some subsystems before others. Therefore, various sequences of subsystem fractionation that evolve a traditional spacecraft to a totally fractionated spacecraft can be defined. The one chosen in this study is based on today's technology maturity level and is presented in Figure 4 . The focus is not on a particular technology but more on a capability that could be reasonably implemented using different technologies.

The $0 \%$ fractionation level corresponds to the traditional monolithic spacecraft. In the first step of the fractionation process, the possibility of wireless data transmission is considered, which leads to the complete fractionation of both the communications and the control and data handling subsystems. For the purposes of this study, the fractionation level of these two subsystems modules is either 0 or $100 \%$. In the second step, the possibility of beaming power is considered, but this time the fractionation can be partial, on a continuum from 0 to $100 \%$ of fractionation, and two different approaches are taken into account: either both the power generation and storage function are fractionated at the same time, or the power generation is entirely fractionated in the power module and only the storage is distributed between the power module and the other modules. Finally, the possibility of collaborative separated positioning is considered, which would lead to the complete fractionation of both the attitude determination and control and the propulsion subsystems.

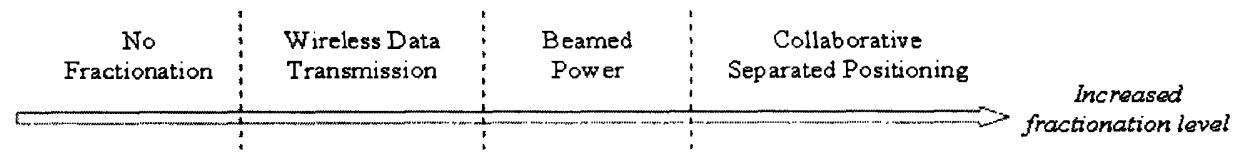

Figure 4: Fractionation technology axis

The second dimension of fractionation at spacecraft level concerns the number of separate modules that form the infrastructure architecture. As the infrastructure can consist of more or fewer modules, this number gives another measure of the spacecraft fractionation level.

\section{B.3.1 Fractionation strategies and architectures matrix}

A matrix of the possible architectures can then be built using those two dimensions as shown on Figure 5. Each box represents a module, and each architecture is labeled with a circled letter from $A$ to $G$. The architectures can be ranked from the least to the most fractionated, i.e. from architecture A to architecture L. Fractionation strategies can be defined as the different paths along the technology axis, from the traditional spacecraft architecture to one of the most fractionated architectures, i.e. architectures $G$ through $L$. As mentioned above, only architectures $\mathrm{D}, \mathrm{E}$, and $\mathrm{F}$ correspond to a continuum along the fractionation paths and are represented by plain lines, whereas the other architectures are represented by dots artificially linked with dotted lines. 


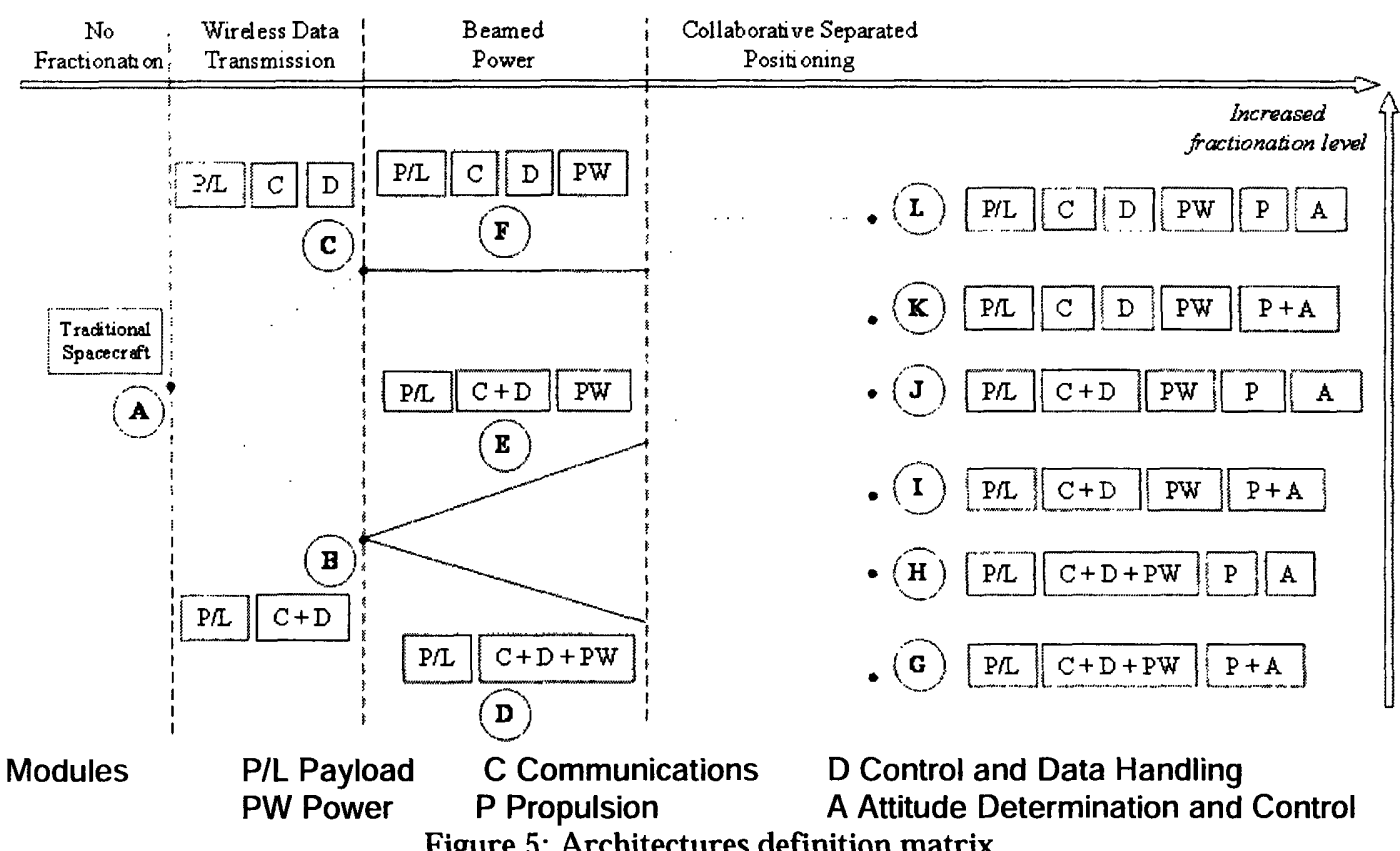

\section{B.3.2 Design parameters at infrastructure level}

If the spacecraft investigated are now assumed to be part of a larger infrastructure, there are additional parameters to take into account in the evaluation. First, the development of a whole infrastructure requires the standardization of the modules, which means a single or a limited number of modules designed to support different payloads. At the spacecraft level, this suboptimality implies that the modules may be oversized for their payload. A standardization parameter, $\mathbf{s}$, simply defined as the percentage of additional payload mass and power requirements the modules could support, is therefore added to describe the architectures investigated. This parameter has an impact on both the spacecraft mass and cost. The production of standardized modules has two major consequences on spacecraft costs: learning effects are expected both in manufacturing and in integration, assembly, and test, and the initial development costs can be divided among all the identical modules. These two effects only depend on the number of units produced, which can reasonably be assumed to be the same for all types of modules. So the number of sets of modules is used as an additional design parameter of the architectures examined. No learning effects for launch costs are taken into account, and a single learning factor is used for all types of modules.

\section{B.3.3 Summary of the architectures design parameters}

Table 2 summarizes all the design parameters that describe the different architectures investigated at spacecraft and at infrastructure level. 


\begin{tabular}{|l|l|}
\hline Design parameters at spacecraft level & \multicolumn{1}{|c|}{$\begin{array}{c}\text { Additional parameters } \\
\text { at infrastructure level }\end{array}$} \\
\hline $\begin{array}{l}\text { - Spacecraft fractionation level } \\
\text { - Number of infrastructure modules } \\
\text { - Power subsystem fractionation approach }\end{array}$ & $\begin{array}{l}\text { - Standardization parameters } \\
\text { Number of units produced }\end{array}$ \\
\hline
\end{tabular}

Table 2: Design parameters at both levels

\section{B.4 Models}

\section{B.4.1 Introduction to the models}

Figure 6 presents the simulation model within the MATE framework and its main elements. The simulation model computes the utility and cost of each architecture investigated for a given mission based on the value of its design parameters.

For each architecture, the system model first computes the mass and cost of the initial spacecraft, as well as the mass, cost and time corresponding to the new spacecraft or module for each attribute scenario. This system model uses as input the design vector and the attributes scenarios, and gives as output the cost and attributes value for each architecture. Second, the utility model uses the attributes value and the utility functions of the customer to compute first the utility derived by the customer from each architecture for each attribute, based on the single-attribute utility functions, and then a single utility figure for each architecture, based on the customer's multi-attribute utility function. The final results of these models are the cost and utility of the different fractionated architectures and of the equivalent traditional spacecraft.

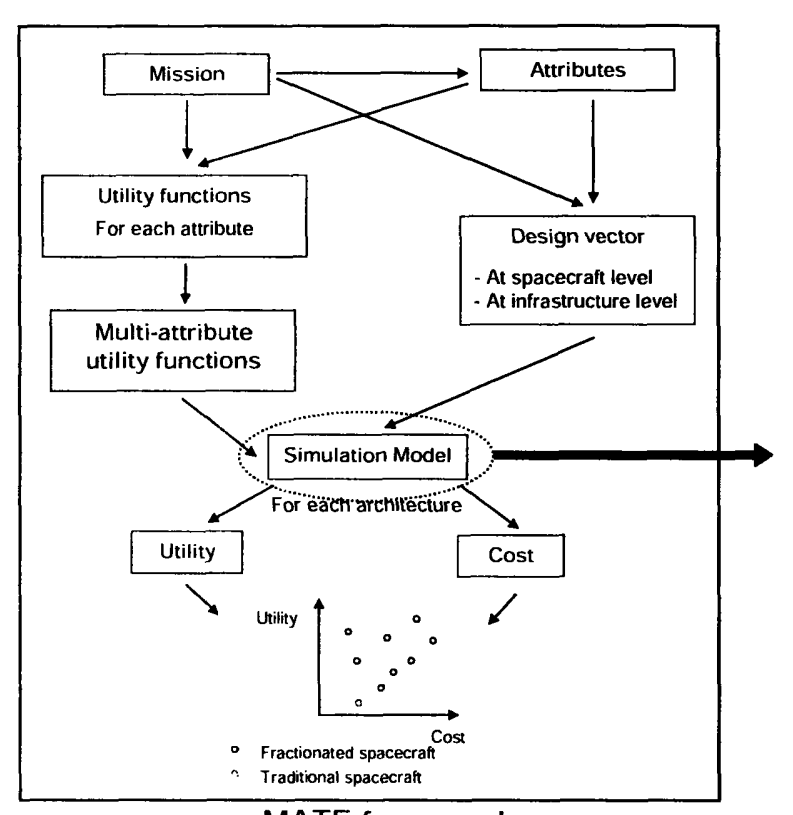

MATE framework

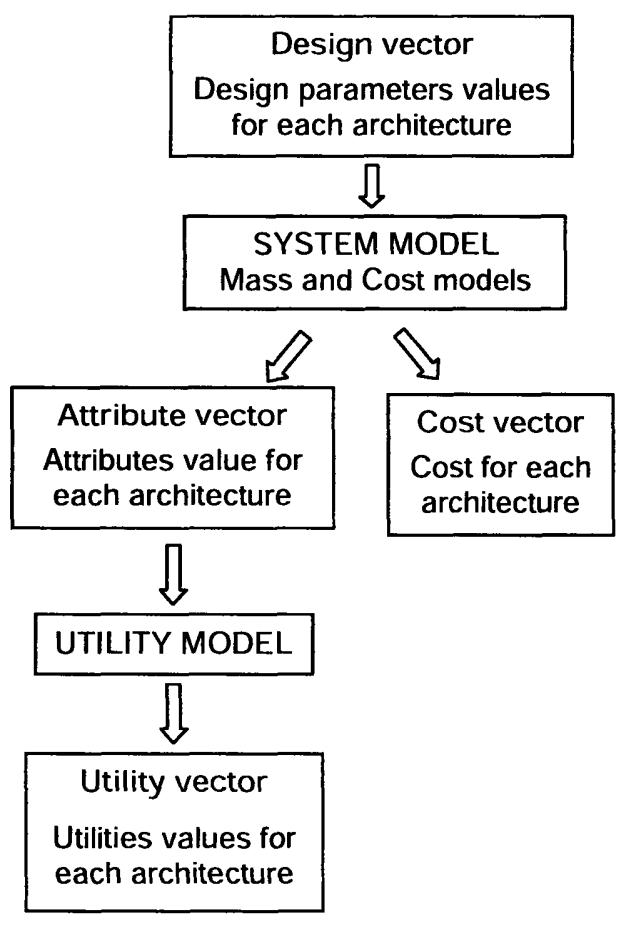

Figure 6: Main elements of the simulation model 


\section{B.4.2 Mass model}

In the mass model, all the architectures are based on identical payloads defined by their mass and power requirements. Given a payload, the first step of the model is to size the corresponding traditional spacecraft. The supporting subsystems are sized using mass ratios and power ratios, as defined in (Wertz and Larson, 1999). Different mass ratios are used depending on the mission application. As mentioned above, three types of applications are investigated: navigation, communications, and sensing missions.

In the model, when a subsystem is fractionated, it is taken out of the spacecraft as it is and becomes the "payload" of a new module. For instance, when the communications subsystem is fractionated, it becomes a communications module with its own power, propulsion, attitude control, and thermal subsystems. The mass ratios are used to size the subsystems of this new module and the power ratios are used to size the power required by these subsystems, given the initial power requirement of the subsystem.

Additional masses and power due to wireless data transmission, power beaming, or collaborative separated positioning are added. For instance, the power module has additional mass and power to beam power to all the other modules and compensate for the losses. They have been modeled as percentages of the masses or powers of the sizing subsystems. For instance, the mass of the wireless data exchange subsystem of the communications module is defined as a percentage of the mass of the communications subsystem. Various technologies have been examined and sensitivity analyses have been run on these parameters.

For comparability purpose, the traditional spacecraft and all the fractionated spacecraft modules are assumed to be designed for the same lifetime, even though one advantage of the fractionation is that modules can be designed with different lifetimes.

The expected results of the mass model are presented in Figure 7 . The different architectures and fractionation strategies defined in Figure 4 are identified by circled letters. Because of the mass model structure, the mass penalty, defined as a percentage of the traditional spacecraft mass, is independent from the mass and power of the payload.

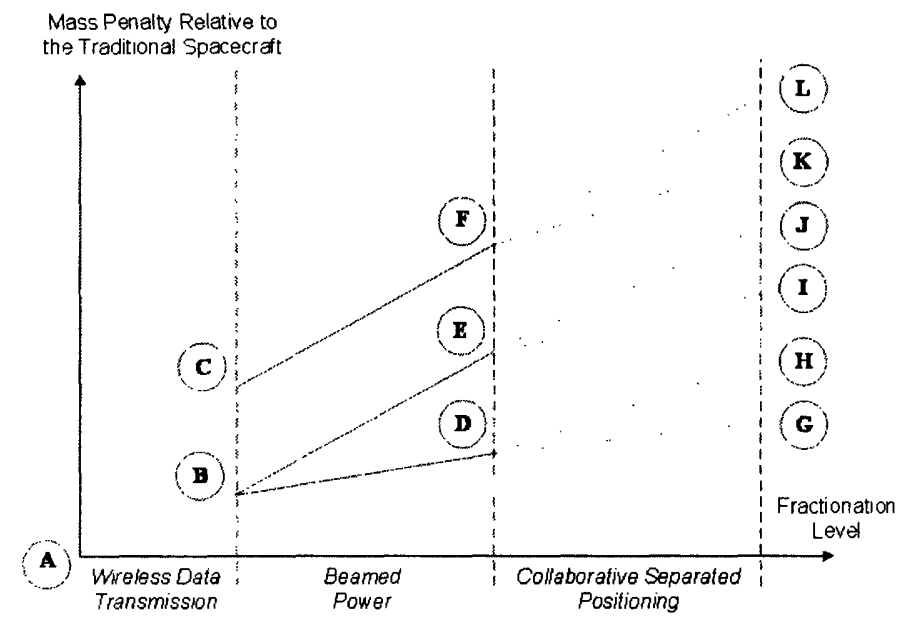

Figure 7: Spacecraft mass increase due to fractionation 


\section{B.4.3 Cost model}

The cost model is built using cost estimating relationships based on subsystem masses ( Wertz and Larson, 1999). A traditional spacecraft cost model, as opposed to a small satellite cost model, is used for both the traditional and the fractionated spacecraft for comparability purpose and because, despite commonalities, the small satellite paradigm remains very different from the fractionated spacecraft one. Nevertheless, traditional cost models can be reasonably considered as unfavorable to the fractionated spacecraft as significant cost reductions are expected, even at spacecraft level, for instance on assembly, integration, and test costs.

The model computes the spacecraft total costs, which includes the development, manufacturing, integration, assembly, and test, and launch costs.

When the fractionated spacecraft are part of an infrastructure, various effects are taken into account in the costs of their recurring elements, i.e. the supporting modules and the platform of the payload module. First, the opposite impacts of standardization and learning are considered in the manufacturing and integration, assembly, and test costs. A unique learning factor, $f$, of 0.85 , which is a typical value in the aerospace industry ${ }^{1}$, is used, according to the following formula (Wertz and Larson, 1999):

With:

$$
\mathrm{C}(\mathrm{N})=\text { TFU.N } \mathrm{N}^{\frac{\ln \mathrm{f}}{\ln 2}}
$$

$\mathrm{C}(\mathrm{N})$ Average cost of the first $\mathrm{N}$ units produced

TFU Theoretical cost of the first production unit

$\mathrm{N} \quad$ Number of units produced

f Learning factor

In addition, the development costs are divided among spacecraft with identical modules, so only a percentage of the initial development effort is assigned to each spacecraft.

The average unit cost of the various fractionated architectures relative to an equivalent traditional architecture are evaluated for different standardization parameters and number of units produced.

\section{B.4.4 Attributes evaluation}

The "ilities" attributes are context- and system- specific, so that their evaluation requires the definitions of general scenarios. Therefore, general scenarios are defined below for each attribute and more specific ones are then defined by the customers interviewed. These general scenarios are used as guideline for the customer interviewed but are not definite as they may not be the most representative of the value of these attributes to a particular customer for some missions.

Maintainability, scalability, and flexibility are measured in monetary units, whereas the corresponding forms of responsiveness are measured in units of time.

\section{B.4.4.1 Maintainability}

To evaluate architecture maintainability, the following general scenario is defined. An internal failure occurs in a subsystem. For fractionated architectures, the module containing the corresponding subsystem is assumed to be exchanged with an identical

\footnotetext{
${ }^{1}$ NASA Learning Curve Calculator, http://wwwl.jsc.nasa.gov/bu2/learn.html
} 
one. For the traditional architecture, a whole identical spacecraft is assumed to be exchanged. The manufacturing cost of a new identical module relative to the cost of manufacturing a new traditional spacecraft is used as a maintainability metric.

\section{B.4.4.2 Scalability}

To evaluate architecture scalability, a new scenario is defined. An increased level of performance is required from the spacecraft, which corresponds to a larger payload requiring more power. The additional performance needed is described by a single parameter $p$, which is defined as a percentage of the initial mass and power requirements of the payload. For fractionated architectures, a new payload module is assumed to be added to the existing one. This new payload module consists of: a payload sized by the increase of performance needed; the corresponding supporting functions that are not fractionable; and the fractionated supporting functions. For instance, the additional power required by this increased performance payload is not provided by an additional infrastructure power module, but by the new payload module itself. This hypothesis reflects the focus of this study on heterogeneous fractionation. Adding an additional power module to compensate for the additional power required would correspond to a homogeneous fractionation approach. However a combination of heterogeneous and homogeneous fractionation is certainly promising in terms of spacecraft scalability and survivability and should be further examined.

If the spacecraft is part of an infrastructure, the new payload module may not include the fractionated supporting functions. Because of standardization, fractionated spacecraft may have oversized supporting functions. Therefore, if additional performance is needed from the supporting functions, these margins may in some cases be sufficient. If they are, the new payload module contains only the payload and the supporting functions not fractionable, both sized by the increase in performance needed. But if they are not within the margins, then additional functions are added to the new payload module to provide the difference between the performance level already supplied by the infrastructure functions and what is needed.

For the traditional architecture, the whole spacecraft is assumed to be exchanged with a new one with the level of performance needed. Both the new payload module and its equivalent traditional spacecraft require a limited new development effort proportional to the parameter $\mathbf{p}$. The development, manufacturing, and launch costs of the new payload module relative to the costs of an equivalent traditional spacecraft are used as a scalability metric.

\section{B.4.4.3 Flexibility}

To evaluate architecture flexibility, a third scenario is defined. A new function is required from the spacecraft.

To distinguish this scenario from the scalability scenario, it is assumed that the new payload has similar requirements as the initial one, so that the initial infrastructure modules can be reused as such. For fractionated architectures, the payload module is exchanged with a newly developed one. For the traditional architecture, the spacecraft is exchanged with a whole newly-developed one. The payload module new development and manufacturing costs relative to the equivalent costs for a whole traditional spacecraft is used as a flexibility metric. 


\section{B.4.4.4 Responsiveness}

The different forms of responsiveness of the architecture are assessed using the same scenarios as the ones used for maintainability, scalability, and flexibility. Firstly, responsiveness associated with maintainability corresponds to the time necessary to get the current function maintained, and therefore to the time necessary to exchange the module that contains the subsystem that failed or the whole spacecraft. The time necessary to develop and manufacture a module relative to the equivalent time for a whole traditional spacecraft is used as a metric for this form of responsiveness. Secondly, responsiveness associated with scalability corresponds to the time necessary to get more of the current function, and therefore to the time necessary to add a new module that or a whole spacecraft. The time necessary to develop and manufacture the additional module relative to the equivalent time for a whole traditional spacecraft is used as a metric for this second form of responsiveness. Finally, responsiveness associated with flexibility corresponds to the time necessary to get a new function delivered, and therefore to the time necessary to exchange the payload module or the whole spacecraft with a new one. The time necessary to develop and manufacture a new payload module relative to the equivalent time for a whole traditional spacecraft is used as a metric for this third form of responsiveness.

\section{B.4.4.5 Summary of the attributes}

Table 3: The general scenarios Table 3 summarizes the general scenario associated with each architecture attribute.

\begin{tabular}{|c|c|c|c|c|}
\hline Attribute & Change & $\begin{array}{l}\text { Module } \\
\text { concerned }\end{array}$ & $\begin{array}{l}\text { Module } \\
\text { needed }\end{array}$ & Attribute metrics \\
\hline Maintainability & \multirow{2}{*}{$\begin{array}{l}\text { Unanticipated } \\
\text { failure of a } \\
\text { subsystem }\end{array}$} & \multirow{2}{*}{$\begin{array}{l}\text { Module } \\
\text { containing the } \\
\text { subsystem } \\
\text { that failed }\end{array}$} & \multirow{2}{*}{$\begin{array}{l}\text { Same } \\
\text { module }\end{array}$} & $\begin{array}{l}\text { Cost to manufacture and } \\
\text { launch a similar module }\end{array}$ \\
\hline $\begin{array}{l}\text { Responsiveness } \\
\text { (Maintainability) }\end{array}$ & & & & $\begin{array}{l}\text { Time to manufacture and } \\
\text { launch a similar module }\end{array}$ \\
\hline Scalability & \multirow{2}{*}{$\begin{array}{l}\text { Increased } \\
\text { demand for } \\
\text { the current } \\
\text { function }\end{array}$} & \multirow{2}{*}{$\begin{array}{l}\text { Payload } \\
\text { module }\end{array}$} & \multirow{2}{*}{$\begin{array}{l}\text { Scaled- } \\
\text { down } \\
\text { additional } \\
\text { module }\end{array}$} & $\begin{array}{c}\text { Cost to develop, } \\
\text { manufacture, and launch an } \\
\text { additional module }\end{array}$ \\
\hline $\begin{array}{l}\text { Responsiveness } \\
\text { (Scalability) }\end{array}$ & & & & $\begin{array}{l}\text { Time to develop, } \\
\text { manufacture, and launch an } \\
\text { additional module }\end{array}$ \\
\hline "Flexibility" & \multirow{2}{*}{$\begin{array}{l}\text { Demand for a } \\
\text { new function }\end{array}$} & \multirow{2}{*}{$\begin{array}{l}\text { Payload } \\
\text { module }\end{array}$} & \multirow{2}{*}{$\begin{array}{l}\text { New } \\
\text { module }\end{array}$} & $\begin{array}{c}\text { Cost to develop, } \\
\text { manufacture, and launch a } \\
\text { new module }\end{array}$ \\
\hline $\begin{array}{l}\text { Responsiveness } \\
\text { (Flexibility) }\end{array}$ & & & & $\begin{array}{c}\text { Time to develop, } \\
\text { manufacture, and launch a } \\
\text { new module }\end{array}$ \\
\hline
\end{tabular}

Table 3: The general scenarios associated with the attributes 


\section{B.4.5 Value models}

For a given mission and a given performance level, the six attributes of each architecture are evaluated using appropriate metrics. The next step in the MATE approach is to translate these attributes, measured in monetary units or in units of time, into the corresponding "value" to a customer of each architecture.

Two approaches are possible depending on the mission. A first approach can only be used when a market exists for the service provided by the spacecraft. A typical application for which this method can be used is commercial telecommunications satellites that generate a revenues stream over their lifetime. The value of the attributes of any architecture can then be translated in terms of revenues.

A second approach is needed to capture the "value" of an architecture to a customer when this value cannot be directly expressed in monetary units. This approach uses utility functions.

\section{B.4.5.1 Net present value model}

- Maintainability

To get a better sense of the value of the maintainability provided by the fractionated spacecraft, the initial and maintenance costs of the fractionated architectures are used to perform net present value (NPV) calculations for various scenarios of failure, as done for instance in (De Neufville and al., 2005).

This part of the model is currently limited to communications satellite as the revenues they generate are definable in monetary units, as a function of the number of transponders. For a given supporting function, a failure profile is defined in the traditional "bathtub" shape, with higher likelihoods of failure in the infant mortality and wear-out periods at the beginning and at the end of the spacecraft lifetime. In case of failure, the decision-maker is assumed to choose to exchange the failing module if the revenues over the rest of the spacecraft lifetime are expected to be larger than the maintenance "costs". The risks associated with such an exchange are not taken into account, but in case of maintenance, only part of the yearly revenue is assumed to be generated. Depending on whether and when the failure occurs, the net present value differs. Given a failure profile, a net present value distribution is obtained for each architecture.

\section{- Scalability}

The initial and upgrade costs of the fractionated architectures are used to perform net present value calculations for different demand scenarios. A simple scenario assumes that at some point in time, the performance level, which after a ramp-up period is assumed constant, changes to a new constant value for the rest of the spacecraft lifetime. To the initial and new performance values are associated different yearly revenues. If the demand increases, the decision-maker may choose to upgrade the spacecraft if the expected revenues over the rest of the spacecraft lifetime are higher than the upgrade costs. As the new level of revenue potentially generated by the spacecraft can equally be lower or higher than the initial one, a normal distribution centered on the initial value with a volatility increasing with time is used.

Depending on when this change occurs and the new revenues that can be potentially generated, different net present values are obtained. 
Thousands of net present value computations are run to get the net present value distribution for each architecture. These distributions are then compared.

- Flexibility

The initial and exchange costs of the fractionated architectures are used to perform net present value calculations for different demand scenarios. A simple type of scenario assumes a constant yearly revenue flow for the initial spacecraft and that at some point in time, a new function potentially generates larger revenues but also simultaneously make the revenues generated by the initial function decrease to a lower level.

If the difference in expected revenues is larger than the exchange costs then the decisionmaker will choose to exchange the payload module.

The level of revenue of the new function was assumed to be independent of the new level of revenue of the original function. As it was assumed that the new payload has the same requirements as the initial one, the difference between the potential revenues generated by the new function and the ones initially generated by the original function has to be within a reasonable range. To each possible scenario is associated a net present value. All new levels of revenues were assumed to be equally probable within given ranges. Thousands of net present value computations are run to get the net present value distribution for each architecture. These distributions are then compared.

\section{B.4.5.2 Utility model}

When no market can be associated with the value to a customer of his space assets, another metrics needs to be used.

- Utility versus value

Two scales can be used to quantify any kind of value: value and utility. Value is an ordinal scale, whereas utility is a cardinal scale, so that utility allows a quantification of the value of each architecture rather than a simple ranking, and its units have meaning relative to each other (De Neufville, 1990). Therefore utility rather than value functions are used to assess the different architectures.

Utility is a special form of cardinal scale, it is an ordered metric scale. This means that, contrary to ratio scale, zero has no absolute meaning, it is simply a reference point. Therefore, ratios between two utility measures do not have any meaning. Moreover, all measurements on an ordered metric scale can be transformed into equivalent measures by a positive linear transformation. It implies that the ends of the utility scale can be arbitrarily chosen, which simplifies utility measurements. An important difference between value and utility is that value corresponds to certainty cases - each alternative evaluated is associated to a certain outcome -, whereas utility correspond to uncertainty cases - each alternative evaluated can only be associated with probabilistic outcomes. This property leads a very useful result for utility: as long as a set of assumptions of his/her preferences hold, a decision maker chooses between uncertain alternatives by comparing their expected utility values. This expected utility theory property was first introduced in (Von Neumann and Morgenstern, 1947). This result is central to utility measurement and decision analysis, and the five axioms necessary to obtain it are presented below. 
The architectural analysis of the fractionated spacecraft is a multi-dimensional problem under uncertainty, therefore the assessment of the different architectures is based on multi-attribute utility theory (Keeney and Raiffa, 1976).

- Single-Attribute Utility theory

A single attribute-utility function, $U(X)$, describes the utility associated with the value of an attribute $X$. As mentioned above, the scale of this utility function is defined by means of two arbitrary points. Usually utility is scaled from 0 to 1 , and the minimum value, 0 , is assigned to the worst level of $\mathrm{X}, \mathrm{X}_{*}$, and the maximum value, 1 , to the best level of $\mathrm{X}^{*}$. Thus:

$$
\begin{gathered}
\mathrm{X}_{*} \leq \mathrm{X} \leq \mathrm{X}^{*} \text { or } \mathrm{X}^{*} \leq \mathrm{X} \leq \mathrm{X}^{*} \\
0 \leq \mathrm{U}(\mathrm{X}) \leq 1 \\
\mathrm{U}\left(\mathrm{X}^{*}\right)=0 \\
\mathrm{U}\left(\mathrm{X}^{*}\right)=1
\end{gathered}
$$

The existence of any utility function depends on six main axioms (De Neufville, 1990). The first assumption necessary is the axiom of completeness (existence of relative preferences for all outcomes). The second assumption is the transitivity axiom (preferences are transitive). The third assumption is the monotonicity axiom (more of a benefit is better). The fourth and fifth assumptions deal with probabilities. They state that probabilities exist and can be quantified and a higher probability of a benefit is better. These five axioms are rather straightforward and are reasonable in many cases. The last assumption necessary to the applicability of utility functions is key but also controversial. The independence or substitution axiom states that:

If a person places an equal value on two possible outcomes, $\mathrm{A}$ and $\mathrm{B}$, then these can be substituted for each other in any choice involving uncertain outcomes without changing that choice. Thus if $\mathrm{A} \sim \mathrm{B}$, then $\mathrm{P}(\mathrm{A})+(1-\mathrm{P}) \mathrm{C} \sim \mathrm{P}(\mathrm{B})+(1-\mathrm{P}) \mathrm{C}$. (De Neufville, 1990)

This axiom leads to the assertion that a person's preferences are linear in probability. However repeated experiments demonstrate that people often act as if their preferences were nonlinear in probability, which lead to the controversy about this axiom.

Recent research indicates that the axiom holds, at least as a first-order approximation, except when the probability of some great consequence is either very small or very high.

It follows from these axioms that the utility of a situation under uncertainty, or lottery, is the sum of the probability of each outcome times the utility of these outcomes, or:

With:

$$
\mathrm{U}(\text { lottery })=\Sigma \mathrm{P}_{\mathrm{i}} \mathrm{U}\left(\mathrm{X}_{\mathrm{i}}\right)
$$

U(lottery) the utility associated with a given lottery

$\mathrm{P}_{\mathrm{i}} \quad$ the probability of the outcome $\mathrm{X}_{\mathrm{i}}$

$\mathrm{U}\left(\mathrm{X}_{\mathrm{i}}\right)$ the utility associated with the outcome $\mathrm{X}_{\mathrm{i}}$

- Multi-attribute utility theory

To get a single utility figure that depends on several attributes, multi-attribute methods must be used. The multi-attribute utility theory is based on two main assumptions about 
the structure of preferences: the preferential independence and the utility independence assumptions (De Neufville, 1990).

Preferential independence assumes that the ranking of preferences over any pair of attributes is independent of the level of the other attributes.

Utility independence assumes that the indifference between a lottery and a certainty equivalent for any attribute does not depend on the levels of the other attributes, which implies that it is possible to measure the utility changes due one attribute, independent of all other attributes. If the utility independence assumption is not satisfied for several attributes, analytical techniques exist to solve this problem. For instance aggregate variables based on the dependent attributes can be defined.

If both of these assumptions hold, the multi-attribute function is defined as in (De Neufville, 1990) by:

With:

$$
\mathrm{KU}(\underline{\mathrm{X}})+1=\Pi\left(\mathrm{Kk}_{\mathrm{i}} \mathrm{U}_{\mathrm{i}}\left(\mathrm{X}_{\mathrm{i}}\right)+1\right)
$$
$\underline{X} \quad$ Vector of the attributes $\mathrm{X}_{\mathrm{i}}$
$\mathrm{U}(\underline{\mathrm{X}})$ Multi-attribute utility function
$\mathrm{U}_{\mathrm{i}}\left(\mathrm{X}_{\mathrm{i}}\right)$ Utility function of the attribute $\mathrm{X}_{\mathrm{i}}$
$\mathrm{k}_{\mathrm{i}} \quad$ Individual scaling factor of the attribute $X_{i}$
$\mathrm{K} \quad$ Normalizing parameter

The scaling factors $\mathrm{k}_{\mathrm{i}}$ can be understood as the attributes importance relative to each other. The normalizing parameter $\mathrm{K}$ ensures that the multi-attribute utility function varies between 0 and 1 . If all the attributes $X_{i}$ are at their best values, then all the $U_{i}\left(X_{i}\right)$ are equal to 1 , and so should $\mathrm{U}(\underline{\mathrm{X}})$. It yields:

$$
\mathrm{K}+1=\Pi\left(\mathrm{Kk}_{\mathrm{i}}+1\right)
$$

\section{Utility Measurement}

This chapter first introduces utility theory and utility measurement process. Then it presents how this process was implemented in this study. Finally, it summarizes the results of two utility interviews with experts in communications and navigation systems. These experts were chosen to represent the military users community of these two systems.

\section{C.1 Theory}

The multi-attribute utility measurement process consists of the following steps as defined in (De Neufville, 1990):

- Specification of the attributes

- Verification of the preference and utility independence assumptions

- Measurement of the single-attribute utility functions

- Measurement of the scaling factors $\mathrm{k}_{\mathrm{i}}$

- Calculation of the normalizing parameter $\mathrm{K}$

- Determination of the multi-attribute utility function 


\section{C.1.1 Specification of the attributes and verification of the assumptions}

First of all, the attributes of the problem need to be defined. Decision-makers have needs, and what is assessed with utility functions are the different ways to satisfy these needs. The attributes represent the different dimensions of the value derived by decision-makers from each solution. Thus attributes are chosen in collaboration with the decision-maker to make sure they correspond to the features that are most valuable to him/her. Once the attributes are defined, the decision-maker has to define its most and least acceptable level of each attribute in the context of the problem. Second, the assumptions of preference and utility independence need to be verified by asking a set of questions to the decisionmakers. Utility independence can also be checked during the determination of the singleattribute utility functions.

\section{C.1.2 Utility measurement}

Once the attributes are defined and the assumptions verified, the single-attribute utility functions need to be measured for each attribute.

Utility measurement is based on two properties presented above: first, that the utility of a lottery is the sum of the utility of the outcomes times their probabilities, and second on the expected utility theory property, i.e., people make decisions in order maximize their utility.

To determine his/her utility function for a given attribute $\mathrm{X}$, the decision maker is interviewed. To measure utility, the use of Lottery Equivalent Probability (LEP) method is recommended by experts, as it overcomes the practical defects of the certainty equivalent method that was widely used (De Neufville, 1990). In practice, LEP method seeks to determine the indifference point of the decision maker between two alternative situations under uncertainty (or lotteries). Each lottery is defined by two outcomes and two corresponding probabilities. Generally, the decision maker has to choose the one he prefers between the two lotteries like the ones that follow. The first lottery, lottery A, corresponds to $\mathrm{P}$ chance of getting the "best" outcome $\mathrm{X}^{*}$ and (1-P) chance of getting the "worst" outcome $\mathrm{X}_{*}$. The second lottery, lottery $\mathrm{B}$, corresponds to $50 \%$ of getting an outcome $\mathrm{X}_{\mathrm{i}}$ (with $\mathrm{X}_{\mathrm{i}}$ between $\mathrm{X}^{*}$ and $\mathrm{X}_{*}$ ) and $50 \%$ chance of getting the "worst" outcome $\mathrm{X}_{*}$. These lotteries can be represented by:

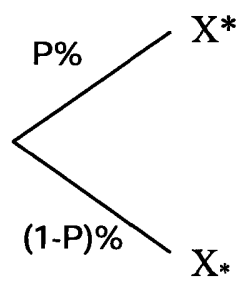

Lottery A

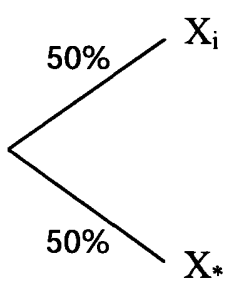

Lottery B

Figure 8: Format of the lotteries used for measuring single-attribute functions

For each $X_{i}$, the probability $P$ is adjusted to determine the decision-maker's indifference point, or lottery equivalent, and its corresponding lottery equivalent probability $\mathrm{P}_{\mathrm{i}}$. The indifference statement can be formally represented by:

$$
\left(\mathrm{X}^{*}, \mathrm{P}_{\mathrm{i}} ; \mathrm{X}_{*},\left(1-\mathrm{P}_{\mathrm{i}}\right)\right) \sim\left(\mathrm{X}_{\mathrm{i}}, 50 \% ; \mathrm{X}_{*}, 50 \%\right)
$$


At equivalence, the decision-maker's expected utilities are equal. Therefore, according to utility theory:

$$
P_{\mathrm{i}} U\left(X^{*}\right)+\left(1-P_{i}\right) U\left(X_{*}\right)=0.5 U\left(X_{i}\right)+0.5 U\left(X^{*}\right)
$$

And as $\mathrm{U}\left(\mathrm{X}^{*}\right)=1$ and $\mathrm{U}\left(\mathrm{X}^{*}\right)=0$ :

$$
\mathrm{U}\left(\mathrm{X}_{\mathrm{i}}\right)=2 \mathrm{P}_{\mathrm{i}}
$$

The pairs $\left(\mathrm{X}_{\mathrm{i}}, \mathrm{P}_{\mathrm{i}}\right)$, for different values $\mathrm{X}_{\mathrm{i}}$ of the attribute $\mathrm{X}$, define the decision-maker's utility function for the attribute $\mathrm{X}$.

One set of lottery-equivalent questions need to be asked per attribute. The number of questions for each single-attribute function depends on the number of points on the utility curve that ones wants to determine for each attribute, as well as on the method used to determine the lottery equivalent probabilities. The standard approach used by the interviewer to determine the lottery equivalence is called "bracketing" (De Neufville, 1990). Rather than asking him/her directly to determine the probability $P_{i}$ for which he/she is indifferent between two lotteries, this method helps the decision-maker to gradually focus on the equivalence. The interviewer starts with a probability that is obviously too high so that the choice between both lotteries is easy for the decisionmaker. Next, the interviewer suggests a probability that is probably too low. Again the answer is easy. In this way, the interviewer has already narrowed down the range of probability. The interviewer continues with this process until the decision-maker settles on the equivalent. This "bracketing" method helps the decision-maker to gradually find the equivalence and avoids the problem of "anchoring", which is a tendency people have to give a high answer when this answer is approached from above and a low answer when it is approached from below.

\section{C.1.3 Scaling factors measurement}

Once the single-attribute utility functions are determined, the scaling factor of each attribute needs to be measured. The scaling factors are measured by finding the indifference point of the decision-maker between two alternative situations (De Neufville, 1990).

Contrary to the utility functions measurement, both situations are not lotteries; the first one is a certain situation. If the scaling factor $k_{i}$ of the attribute $X_{i}$ is measured, in this first certain situation, the attribute $\mathrm{X}_{\mathrm{i}}$ is at its best level while the other attributes are at their worst level. The second situation corresponds to the $\mathrm{P} \%$ chance of getting all the attributes at their best level, and (1-P)\% chance of getting all the attributes at their worst level. The indifference point is determined using "bracketing" by adjusting the probability $\mathrm{P}$ until the equivalent probability $\mathrm{P}_{\mathrm{i}}$ is reached. The indifference statement can be formally represented by:

$$
\left(\mathrm{X}_{\mathrm{i}}^{*}, \mathrm{X}_{\mathrm{j}} *\right) \sim\left(\underline{\mathrm{X}}^{*}, \mathrm{P}_{\mathrm{i}} ; \underline{\mathrm{X}} *,\left(1-\mathrm{P}_{\mathrm{i}}\right)\right) \text { for all } \mathrm{j} \neq \mathrm{i}
$$

The scaling factor $k_{i}$ is simply equal to $P_{i}$. Thus one set of questions is needed for each scaling factor.

\section{C.1.4 Determination of the multi-attribute function}

Once the scaling factors $\mathrm{k}_{\mathrm{i}}$ are measured, the normalizing parameter $\mathrm{K}$ is calculated using the formula presented above:

$$
\mathrm{K}+1=\Pi\left(\mathrm{Kk}_{\mathrm{i}}+1\right)
$$


When more than two attributes are taken into account, $\mathrm{K}$ must be determined by trial and error.

The final step is the determination of the multi-attribute utility function. As presented above, the utility function $\mathrm{U}(\underline{\mathrm{X}})$ is defined by:

$$
\mathrm{KU}(\underline{\mathrm{X}})+1=\Pi\left(\mathrm{Kk}_{\mathrm{i}} \mathrm{U}_{\mathrm{i}}\left(\mathrm{X}_{\mathrm{i}}\right)+1\right)
$$

The determination of the multi-attribute utility function is straightforward when the single-utility function $U_{i}\left(X_{i}\right)$ are accurately defined for each $X_{i}$ of interest. Otherwise, the single-utility functions need to be interpolated, using standard formulas to find the best fit. Then these expressions are directly introduced in the formula for the multi-attribute utility.

\section{C.2 Implementation}

All the steps described in the previous section were implemented in a three-step process. Each interviewee goes first through a preliminary questionnaire and second through the utility interview itself. Finally, each interviewee reviewed the results of his/her interview for validation. This process was standardized so that, for a same mission, answers from different interviewees can be compared, and so that it can be used for different missions in the different fields of application.

The goal of this overall study, and in particular the fact that these utility interview results would be used to assess fractionated and traditional spacecraft architectures, were not revealed to the interviewees before the end of the interview, to avoid to influence on their answers.

\section{C.2.1 Preliminary questionnaire}

\section{C.2.1.1 Attributes specification and the need for a preliminary questionnaire}

One of the contributions of this study is that it investigates only non-traditional performance attributes. This has two main consequences on the implementation of the utility measurement. First, the attributes of interest are defined in advance and later discussed and validated with the decision-maker. Second, the nature of the attributes requires the definition of scenarios for each of them in collaboration with the decisionmaker.

First, in this study, different spacecraft architectures are evaluated and compared. Therefore, the attributes chosen as evaluation criteria for the different architectures are meant to reflect the value derived from space assets by a potential customer and allow a fair comparison of traditional and fractionated spacecraft in the same trade-space. The values of the attributes should significantly vary between the traditional and fractionated spacecraft but also between the various fractionated spacecraft architectures. Usually the attributes are chosen in collaboration with the decision-maker to ensure it correspond to the features of main value for him/her. But this study seeks to measure the variation in value delivered to the customer by different architectures at a given performance level, and the attributes considered were therefore limited to non-traditional performance parameters. Thus a list of attributes (maintainability, scalability, flexibility, and the 
corresponding forms of responsiveness) was first defined and then was submitted to the customer to discuss their relevance to him/her.

Second, the nature of the attributes requires the definition of scenarios to measure singleutility functions. The utility derived by customer from flexibility cannot be evaluated as such, as it can be done with traditional performance parameters. The various forms of flexibility are relative (Nilchiani, 2005). Therefore, for each mission and for each form of space assets flexibility investigated, scenarios that best represent the potential value of this type of flexibility need to be defined with the decision-maker.

\section{C.2.1.2 The preliminary questionnaire}

Therefore a preliminary questionnaire was needed to:

- Introduce the six forms of space assets flexibility investigated

- Validate the relevance of these attributes with the decision-maker

- Define for each attribute a scenario that best capture the potential value of the attribute to the decision-maker

- Get a sense of the absolute and relative values of the attributes to the decisionmaker qualitatively

- Validate the preference and utility independence assumptions

The preliminary questionnaire was developed in a standard format to capture of the preferences of any decision-maker in any field of application. This questionnaire is presented in Appendix.

\section{C.2.2 Utility interviews}

\section{C.2.2.1 Scenarios}

In the preliminary questionnaire, each interviewee defines one scenario per attribute, and then defines the most and least acceptable value of the attribute for this scenario.

For each interviewee, this scenario will correspond to a single utility function. But this utility function is one among several ones that correspond to different possible scenarios for a same attribute.

For the attribute of maintainability, the decision-maker associate different utility curves to different failure scenarios, as shown in Figure 9. For a given maintenance cost, the decision-maker's utility is likely to increase with the severity of the failure scenario. The severity of the failure scenario depends on when the failure occurs and on the severity of the failure of the system (i.e., if the failure is partial or complete). 


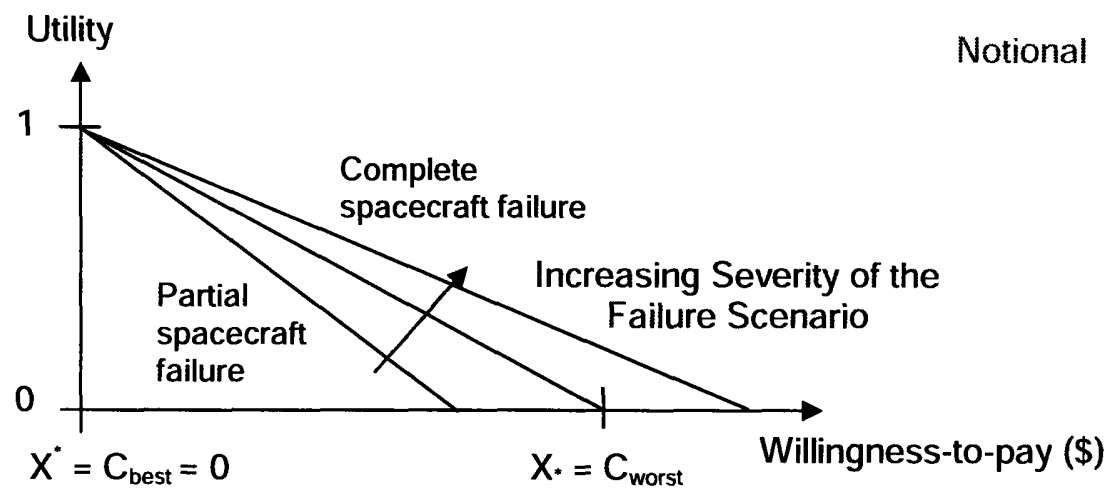

Figure 9: Utility versus cost to maintain for different failure scenarios

For the attribute of scalability, the decision-maker has different utility functions depending on the additional capacity it can get, as shown on Figure 10. For a given cost, one can expect the decision-maker's utility to increase with the amount of additional capacity.

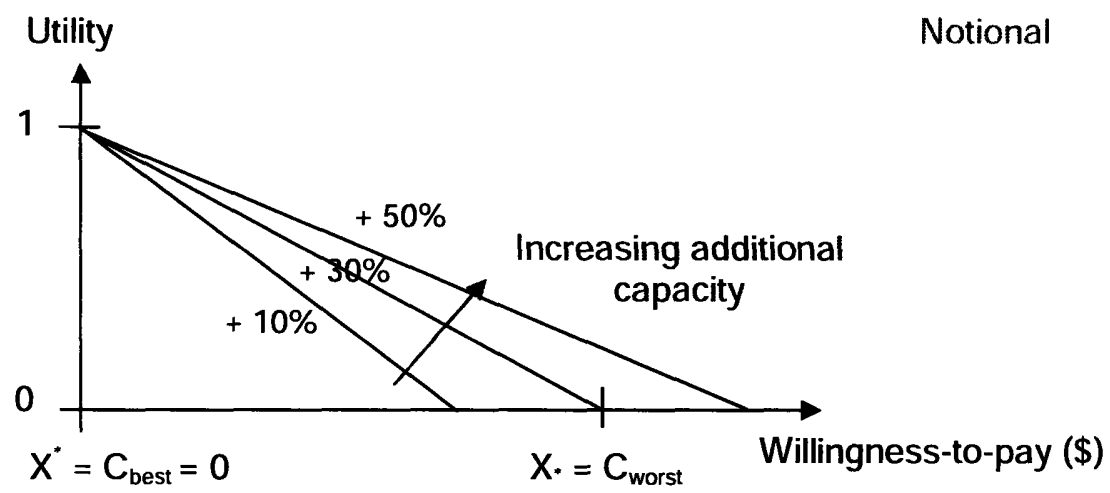

Figure 10: Utility versus upgrade costs for different scenarios

For the attribute of flexibility, the decision-maker has different utility functions depending on the new functionality it can get, as shown on Figure 11. If the possible new functionalities are ranked according to the decision-maker's preferences, for a given cost, one can expect its utility to increase from least valued to the most valued new functionality. 


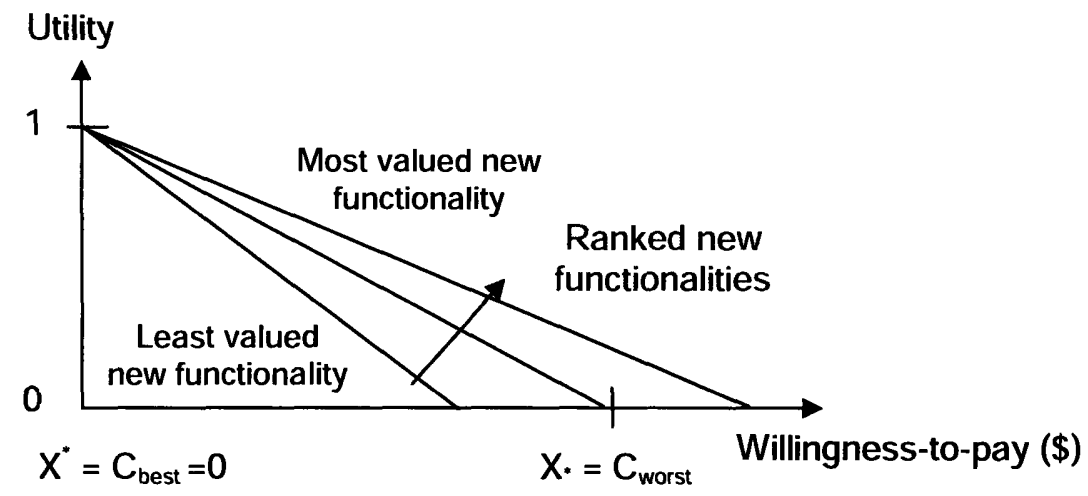

Figure 11: Utility versus upgrade cost for different scenarios

Similar notional figures could be plotted to explain the same phenomena for the three forms of responsiveness, but with time instead of willingness-to-pay on the x-axis.

All these different utility functions could be measured, but this study tried to focus on the scenarios that best represent the actual needs of space systems customers. The choice of these scenarios with the interviewee depend on both probability and value of his/her needs. When considering the need for maintenance, a reasonable worst case scenario was chosen, to fully represent the value of maintainability. When considering the need for more of the current function, first the most valued parameter of performance was chosen, and then the most probable level of additional capability needed in the close future was defined. Finally, when considering the need for a new function, the most valued new function was chosen.

The scenarios defined to measure the value of the three forms of flexibility assessed in monetary units (maintainability, scalability, flexibility) are the same as the ones used to measure the value of the corresponding forms of responsiveness. It means that the scenario defined to measure the value of maintainability to the interviewee is the same as the one used to measure the value of responsive maintenance. Thus the interviewee needs to define only three scenarios for the six forms of flexibility.

It is important to keep mind that these scenarios were defined with a focus on the space segment value proposition. The "ilities" in these scenarios should come mostly from the space segment, as different architectures of the space segment only are investigated. More generally, when defining the scenarios, even though the interviewee should not be influenced in his/her choices, it is crucial to keep in mind the goal of the utility interviews. In this study, the scenarios will be used to link the attributes metrics, as defined in the simulation model, and the utility figure associated with each architecture, so that the attributes metrics should be meaningful to the defined scenarios and viceversa.

\section{C.2.2.2 Single-attribute utility functions measurements}

During the preliminary questionnaire, for each single-attribute function, the decisionmaker defines the least and most acceptable values of the attribute, which are respectively assigned to a utility of 0 and 1 , so that two points on the utility curve are already defined before the utility interview. The number of additional points that are measured during the interview has to be chosen by the interviewer. It is a trade-off between the duration of the 
interview and the desired accuracy of the results. Besides, the interviewer needs to choose the values of the attributes for which these additional points are measured. These values can be chosen at regular intervals in the attribute range, but in some case there may be points of particular interest on the attribute utility curve, such as inflexion points. Such singularities have to be investigated during the preliminary discussion.

Then, for each value of the attribute investigated, the interviewer seeks to determine when the decision-maker is indifferent between two lotteries. Each of these lotteries is defined by two possible outcomes and their corresponding probabilities. An example of the format of the questions asked during the utility interviews is presented below. This question measures the value derived by a customer from the possibility of maintaining its satellite at different costs, or stated differently, it measures the customer willingness-topay for the maintenance of its satellite. A satellite and its failure scenario are initially defined, and during the preliminary questionnaire the decision-maker defined the most and least acceptable costs to maintain this satellite in this scenario, $\mathrm{C}_{\text {best }}$ and $\mathrm{C}_{\text {worst }}$.

"Which of the following situations do you prefer?

Situation A) $\quad 20 \%$ chance of maintaining the current function for $\mathrm{C}_{\text {best }} \& 80 \%$ chance of maintaining the current function for $\mathrm{C}_{\text {worst }}$

Situation B) $50 \%$ chance of maintaining the current function for $3 / 4$ of $\mathrm{C}_{\text {best }} \&$ $50 \%$ chance of maintaining the current function for $\mathrm{C}_{\text {worst }}$ "

A visualization method was developed in MS Excel to help the interviewee answering these lottery questions. The questions were presented to the interviewee in the format shown in Figure 12.

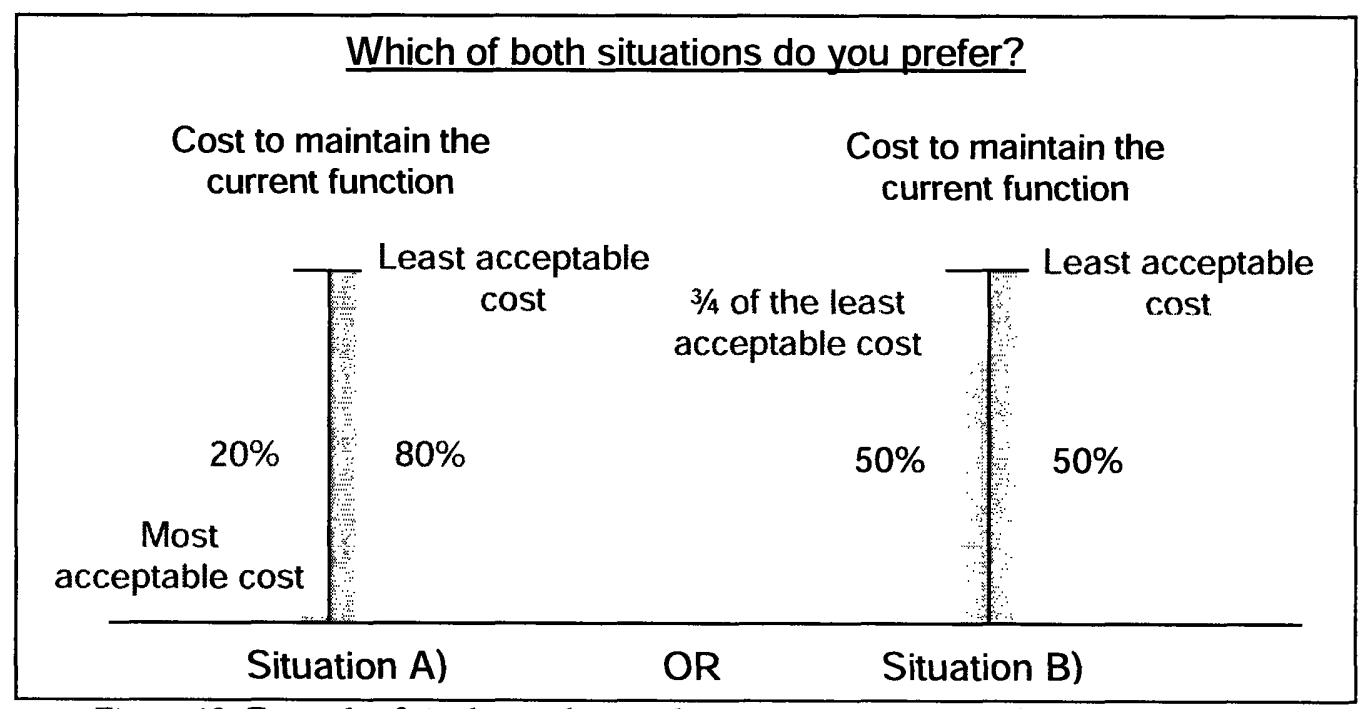

Figure 12: Example of single-attribute utility question as presented to the interviewee

The percentages in situation $\mathrm{A}$ ) correspond to $\mathrm{P} \%$ and $(1-\mathrm{P}) \%$ and are varied using "bracketing" until the lottery equivalent probability is reached. As explained above, the utility corresponding to the value chosen for the attribute (in the example above $3 / 4$ of the least acceptable cost) is twice the lottery equivalent probability measured. Similar questions are asked for each attribute and for each value of the attributes investigated. 


\section{C.2.2.3 Scaling factors measurements}

Once the single-attribute functions are determined, the scaling factors need to be measured. An example of the format of the questions asked is presented below.

This question measures the scaling factor of the maintainability attribute. Scenarios have been defined for each forms of flexibility, as well as the ranges of all the attributes for these scenarios.

"Which of the following situations do you prefer?

Situation A) Maintaining the current function for $\mathrm{C}_{\text {best }}$ in $\mathrm{T}_{\text {worst }}$ \&getting $\mathrm{X} \%$ more of the current function for $\mathrm{C}_{\text {worst }}$ in $\mathrm{T}_{\text {worst }} \&$ getting a different function for $\mathrm{C}_{\text {worst }}$ in $\mathrm{T}_{\text {worst }}$ "

Situation B) $20 \%$ chance of maintaining the current function for $\mathrm{C}_{\text {best }}$ in $\mathrm{T}_{\text {best }}$ \& getting $\mathrm{X} \%$ more of the current function for $\mathrm{C}_{\text {best }}$ in $\mathrm{T}_{\text {best }} \&$ getting a different function for $\mathrm{C}_{\text {best }}$ in $\mathrm{T}_{\text {best }}$ AND $80 \%$ chance of maintaining the current function for $\mathrm{C}_{\text {worst }}$ in $\mathrm{T}_{\text {worst }} \&$ getting $\mathrm{X} \%$ more of the current function for $\mathrm{C}_{\text {worst }}$ and time $\mathrm{T}_{\text {worst }}$ \& getting a different function for $\mathrm{C}_{\text {worst }}$ in $\mathrm{T}_{\text {worst }}$ "

To measure the scaling factors, a visualization method similar to the one used for singleattribute utility measurement was developed to help the interviewee answering the questions. The questions were presented to the interviewee in the format shown in Figure 13. 


\section{Which of both situations do you prefer?}

\section{Situation A)}

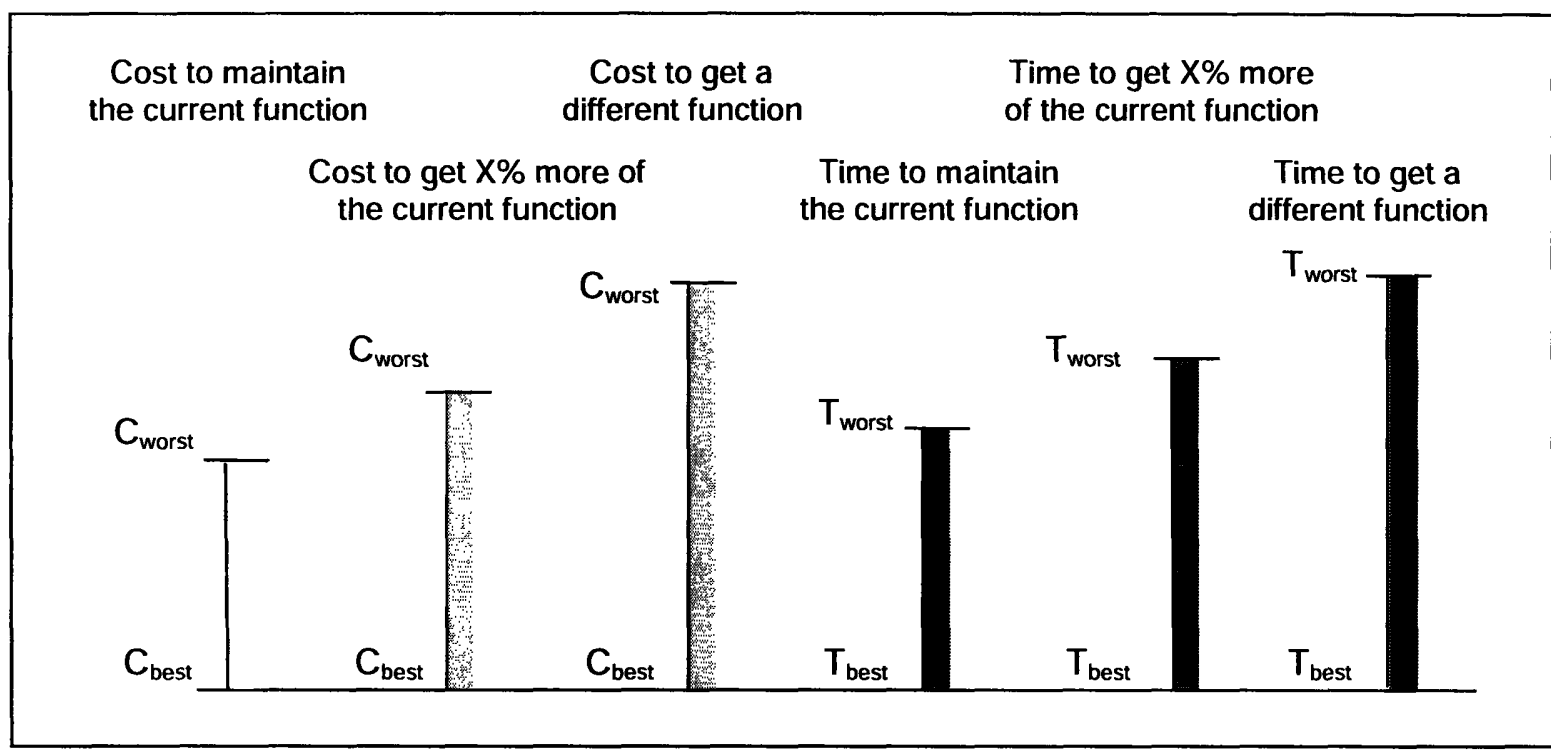

OR

Situation B)

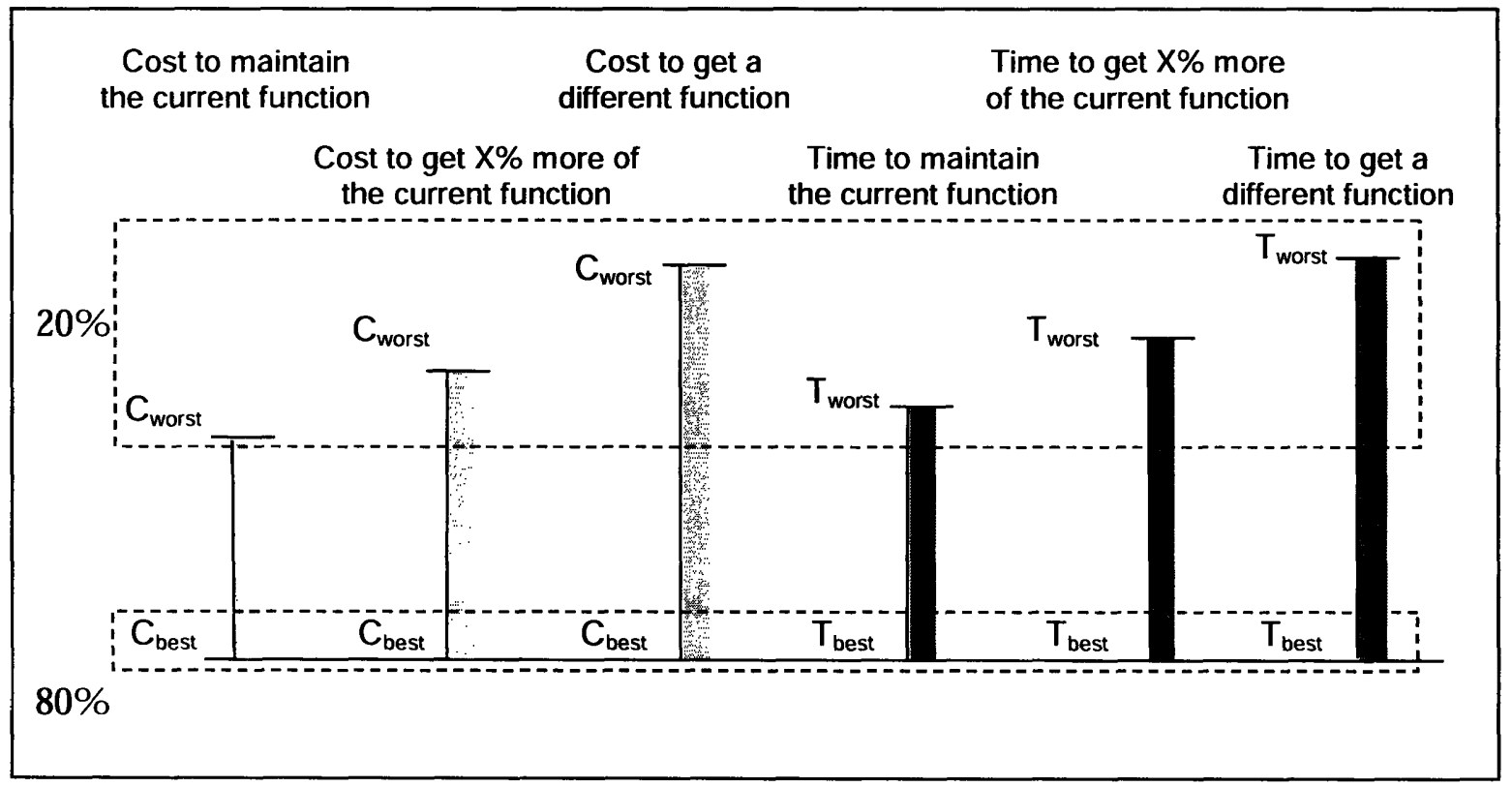

Figure 13: Format of the questions used to measure the scaling factor of the maintainability attribute 
The percentages in situation $\mathrm{B}$ ) correspond to $\mathrm{P} \%$ and (1-P)\% and are varied using "bracketing" until the equivalent probability is reached. As explained above, the scaling factor is equal to the equivalent probability. Similar questions are asked for each attribute.

During the utility interview, the coherence of the interviewee's answers needs to be checked. For the single-attribute utility functions, this verification includes checking that the interviewee's utility remains constant or decreases as cost or time increases, but also checking that the lottery equivalent probability is less than $50 \%$, as utility is twice the equivalent probability and cannot be larger than one in this context.

\section{C.2.3 Validation of the results}

There are many ways to validate the results, and the one chosen depends on many factors, but mainly on the number of attributes and the availability of the interviewee. The ideal case is to have a limited number of attributes and the possibility to run a validation interview. This final interview aims at formally establishing preferential and utility independence and validating the utility functions derived from the utility interview, and it requires three sets of questions in a format similar to the one of the scaling factor measurement questions.

In this study, because of the limited availability of the interviewees and because of the large number of attributes, which make the validation questions very difficult to answer and the potential validation interview rather long, it was decided that for this case no validation interview would be conducted. The preferential and utility independence were discussed during the preliminary interview, and to validate the utility functions, a plot of all the single-attribute utility functions and the scaling parameters were sent to the interviewees. It was possible because the interviewees had a clear understanding of the concept of utility and utility theory.

\section{C.3 Results of the utility interviews}

The results of the utility measurement process for two interviewees are presented below. The first interviewee is an expert in military communications satellites and gives his perception on the utility derived by the military communications satellite users from their space systems. The second interviewee is an expert in navigation satellite and gives his perception on the utility derived by navigation satellite military users from their space systems. Both interviewees were chosen to be as representative as possible of the two users communities and to be able to take a decision-maker perspective on these space systems.

\section{C.3.1 Communications missions}

\section{C.3.1.1 Scenarios}

During the preliminary questionnaire, the communications expert was asked to define a specific scenario for each attribute, which would be most representative of the military communications satellites users' needs. In addition, the interviewee was asked to define the least and worst acceptable cost and time associated with these scenarios. Table 4 summarizes the scenarios and these values. 
The scenario associated with scalability is the possibility of getting satellites with more power and larger transfer wave tubes (TWTs). The scenario associated with flexibility is the possibility of getting better anti-jam capability with a minimum impact on the performance.

First, one can notice that the attributes of maintainability and its corresponding form of responsiveness are not investigated. The interviewee considered that in case of failure, another satellite already on-orbit would be used to quickly compensate for the loss. To him, it is unlikely that users would be willing to wait for the capability to be restored more than a couple of days so that launching a new satellite (or module) would not be an option. Second, the most acceptable - or best - cases were defined as "free" for the attributes measured in monetary units and as "instantaneously" for the attributes measured in units of time. 


\begin{tabular}{|c|c|c|}
\hline 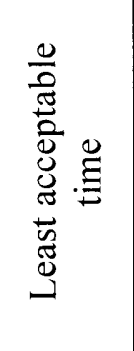 & 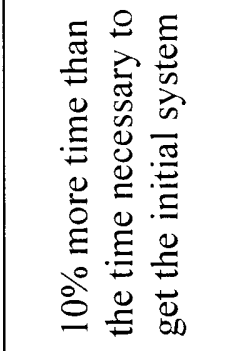 & 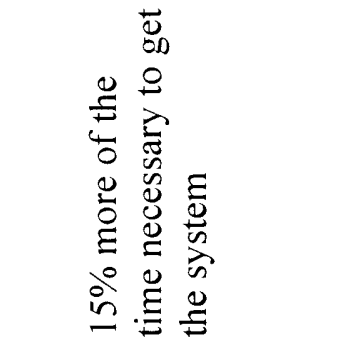 \\
\hline 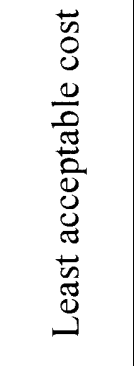 & 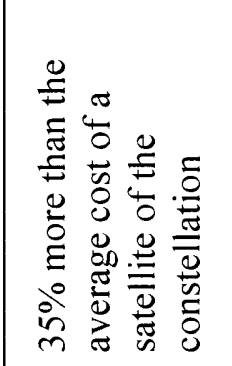 & 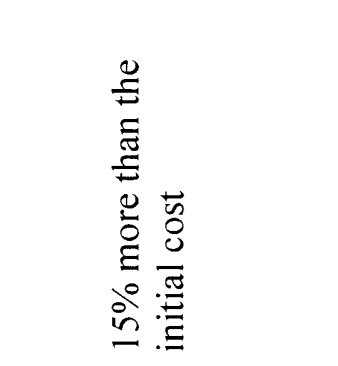 \\
\hline 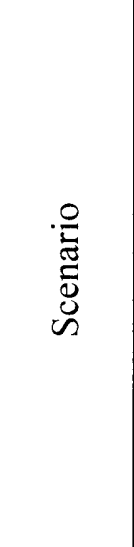 & 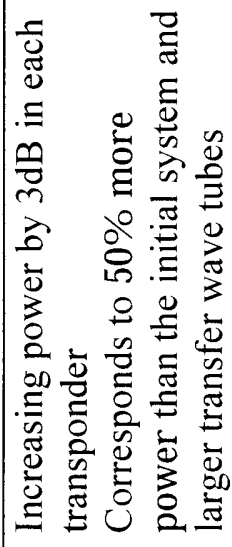 & 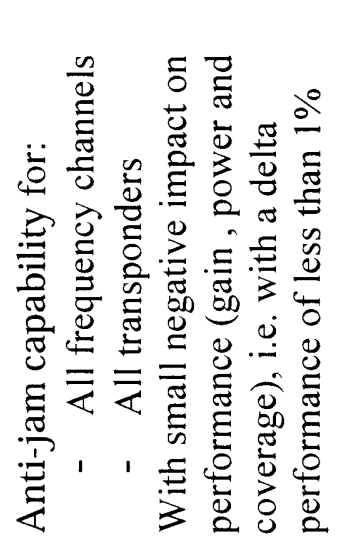 \\
\hline 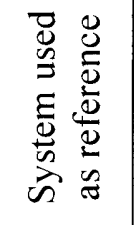 & 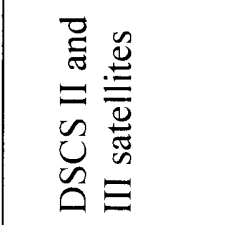 & 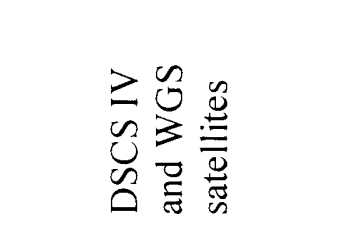 \\
\hline $\begin{array}{l}\stackrel{\vec{E}}{\mathscr{D}} \\
\text { }\end{array}$ & $\begin{array}{l}\vec{D} \\
\stackrel{\vec{I}}{n} \\
\dot{x}\end{array}$ & $\begin{array}{l}\bar{D} \\
\overline{\tilde{J}} \\
\dot{m} \\
\dot{x}\end{array}$ \\
\hline 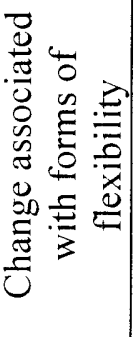 & 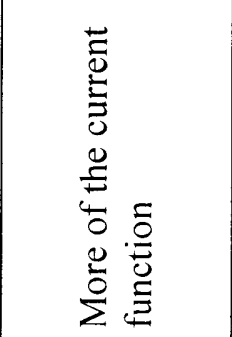 & 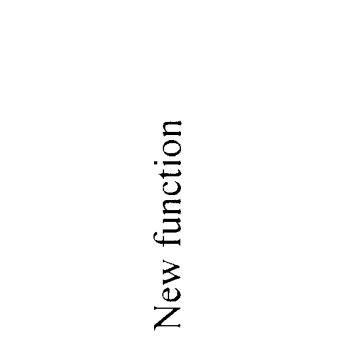 \\
\hline
\end{tabular}


C.3.1.2 Single-attribute utility functions

During the utility interview, the communications expert defined first the single-attribute functions presented below.

Figure 14 shows the utility associated with the attribute of scalability. It presents the relationship between utility and cost to get additional power. The interviewee's utility linearly decreases with the cost to get additional power, from 1 when the additional performance is free to 0 when the additional performance costs $35 \%$ more than the initial satellite cost or more. The linearity of this relationship, and of the following ones, shows that the interviewee is risk-neutral.

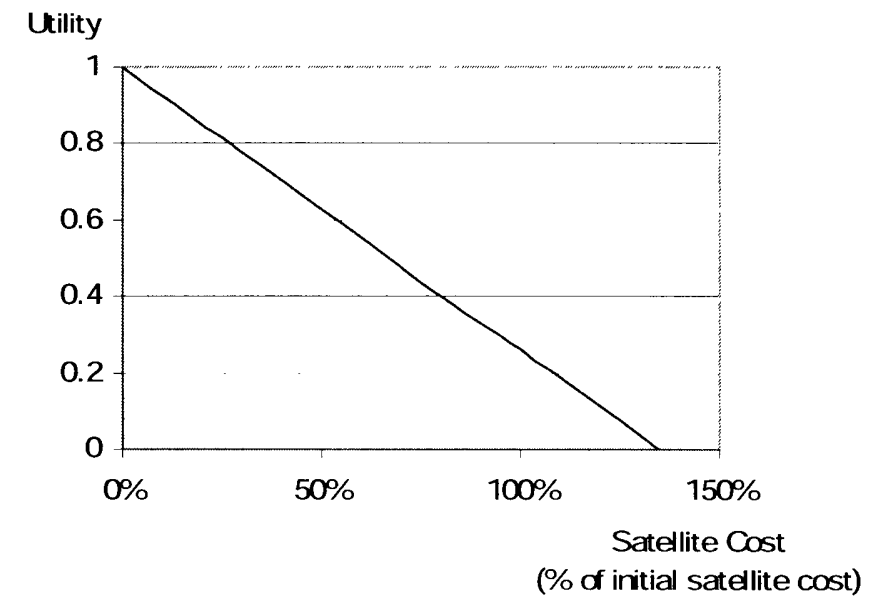

Figure 14: Utility versus satellite cost to get $50 \%$ additional power

If $\mathrm{C}$ is the cost to get the additional performance, the relationship between utility and cost is:

$$
\mathrm{U}_{\text {Scalability }}(\mathrm{C})=1-\frac{\mathrm{C}}{1.35}
$$

Figure 15 shows the utility associated with the attribute of responsiveness corresponding to scalability. It presents the relationship between utility and time necessary to get additional power. The interviewee's utility linearly decreases with the time necessary to get additional power, from 1 when the additional performance is instantaneous to 0 when to get the additional performance it takes $10 \%$ more than the initial satellite delivery time or more. 


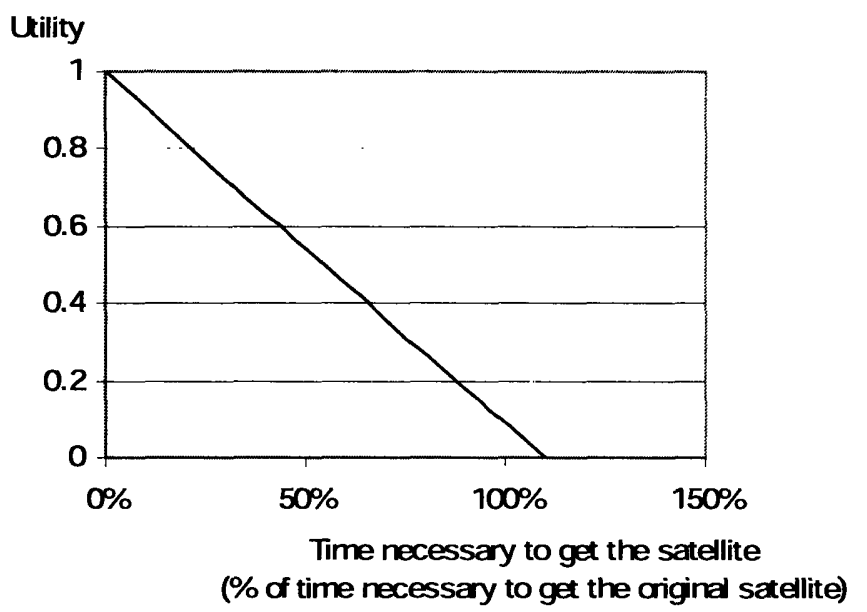

Figure 15: Utility versus time necessary to get $50 \%$ additional power

If $T$ is the time necessary to get the additional performance, the relationship between utility and time is:

$$
U_{\text {Resp. Scal. }}(T)=1-\frac{T}{1.1}
$$

Figure 16 shows the utility associated with the attribute of flexibility. It presents the relationship between utility and cost to get anti-jam capability. The interviewee's utility linearly decreases with the cost to get this capability, from 1 when the additional capability is free to 0 when it costs $15 \%$ more than the initial satellite cost or more.

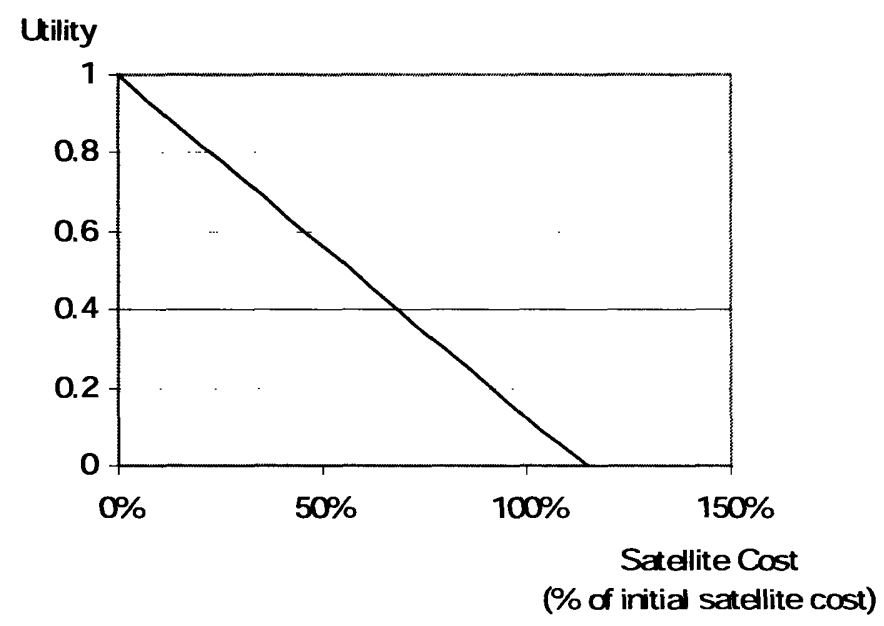

Figure 16: Utility versus satellite cost to get anti-jam capability

If $C$ is the cost to get the new capability, the relationship between utility and cost is:

$$
U_{\text {Flexibility }}(C)=1-\frac{C}{1.15}
$$

Figure 17 shows the utility associated with the attribute of responsiveness corresponding to flexibility. It presents the relationship between utility and time necessary to get the 
anti-jam capability. The interviewee's utility linearly decreases with the time necessary to get this capability, from 1 when the new capability is instantaneous to 0 when to get it it takes $15 \%$ more than the initial satellite delivery time or more.

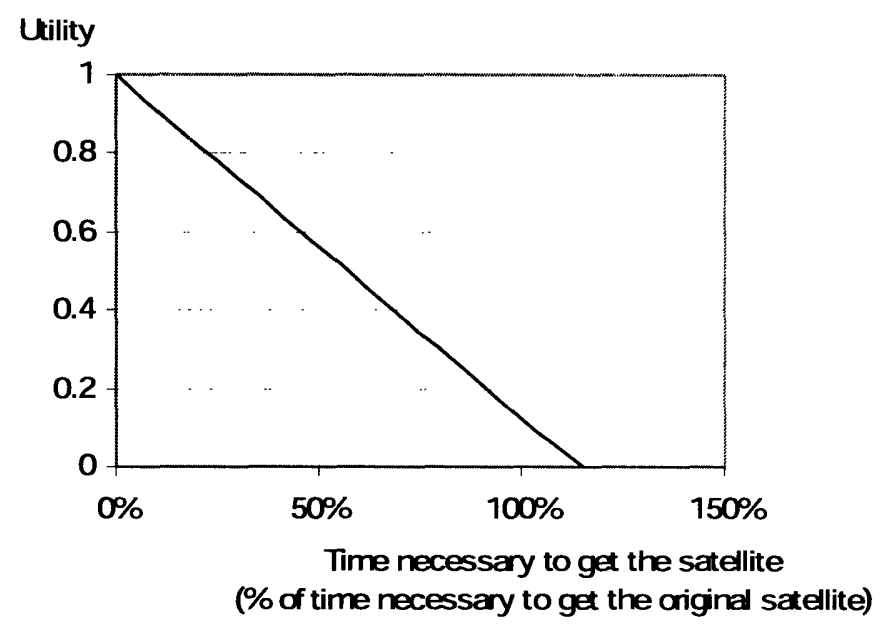

Figure 17: Utility versus time necessary to get anti-jam capability

If $T$ is the time necessary to get the new capability, the relationship between utility and time is:

$$
U_{\text {Resp.Flexi. }}(T)=1-\frac{T}{1.15}
$$

\section{C.3.1.3 Scaling factors}

In addition to the single-attribute utility functions, the scaling factor for each attribute is measured during the utility interview. Table 5 presents the value of these different scaling factors.

\begin{tabular}{|l|l|c|}
\hline \multicolumn{1}{|c|}{ Attribute } & \multicolumn{1}{c|}{ Scenario } & $\begin{array}{c}\text { Weighting parameter in the } \\
\text { utility equation (in \%) }\end{array}$ \\
\hline Scalability & Additional power cheaply & 42.5 \\
\hline Responsiveness & Additional power quickly & 17.5 \\
\hline Flexibility & Anti-jam capability cheaply & 12.5 \\
\hline Responsiveness & Anti-jam capability quickly & 17.5 \\
\hline
\end{tabular}

Table 6 presents the ranking of the attributes by order of importance to the interviewee according to the scaling factors he defined during the utility interview. These results seem consistent with the preferences expressed by the interviewee during the preliminary questionnaire. His perception is that for the military satellite users community scalability is more valuable than flexibility, with the cost factor being significantly more important than the time factor. 


\begin{tabular}{|l|c|}
\hline \multicolumn{1}{|c|}{ Scenario } & $\begin{array}{c}\text { Rank } \\
\text { From the most important (1) to } \\
\text { the least important (4) }\end{array}$ \\
\hline Additional power cheaply & 1 \\
\hline Additional power quickly & 2 \\
\hline Anti-jam capability quickly & 2 \\
\hline Anti-jam capability cheaply & 4 \\
\hline
\end{tabular}

Table 6: Attributes ranking based on the scaling factors

\section{C.3.1.4 Multi-attribute utility function}

The multi-attribute function that represents the utility as a function of the single-utility functions is:

$U(\underline{X})=\frac{1}{0.3464}\left(\left(0.1472 * U_{\text {Scal }}+1\right)\left(0.0606 * U_{\text {Resp.Scal. }}+1\right)\left(0.0433 * U_{\text {Fle }}+1\right)\left(0.0606 * U_{\text {Resp.Flexi. }}+1\right)-1\right)$

With:

$$
\begin{gathered}
U_{\text {Scal }}\left(C_{\text {Scal }}\right)=1-\frac{C_{\text {Scal }}}{1.35} \\
U_{\text {Resp.Scal. }}\left(T_{\text {Scal }}\right)=1-\frac{T_{\text {Scal }}}{1.1} \\
U_{\text {Flexi }}\left(C_{\text {Flexi }}\right)=1-\frac{C_{\text {Flexi }}}{1.15} \\
U_{\text {Resp.Flexi. }}\left(T_{\text {Flexi }}\right)=1-\frac{T_{\text {Flexi }}}{1.15}
\end{gathered}
$$

\section{C.3.2 Navigation}

\section{C.3.2.1 Scenarios}

During the preliminary questionnaire, the navigation expert was asked to define a specific scenario for each attribute, which would be most representative of the navigation satellites military users' needs. In addition, the interviewee was asked to define the least and worst acceptable cost and time associated with these scenarios. Table 4 summarizes the scenarios and these values.

The scenario associated with maintainability is the complete failure of one spacecraft after one year of operations. There is a particular dynamics with navigation satellites, due to the nature of the navigation system. Navigation is possible only if several satellites are available and "properly" positioned, so that 9 satellites in view can be considered as the minimum needed in order to have 5 in view that are properly positioned and can enable users to get their position with an optimum accuracy. This explains why there is a constant management of the navigation systems constellation. The failure of a satellite is not considered as critical if it is the only one to fail, but if a second satellite fails this failure is considered as more critical than the first one, and so on until a fifth satellite fails, which is then considered as a catastrophic failure. Thus the cost decision-makers are willing-to-pay and the time they are willing to wait to maintain a satellite in case of failure is dependent on how many other failures may have occurred in the constellation. 
In this study, the case of failure of a first satellite, which can be considered as a "best case", is taken into account.

The scenario associated with scalability corresponds to additional coverage of both poles. Finally, the scenario associated with flexibility is the possibility of navigating inside buildings. 


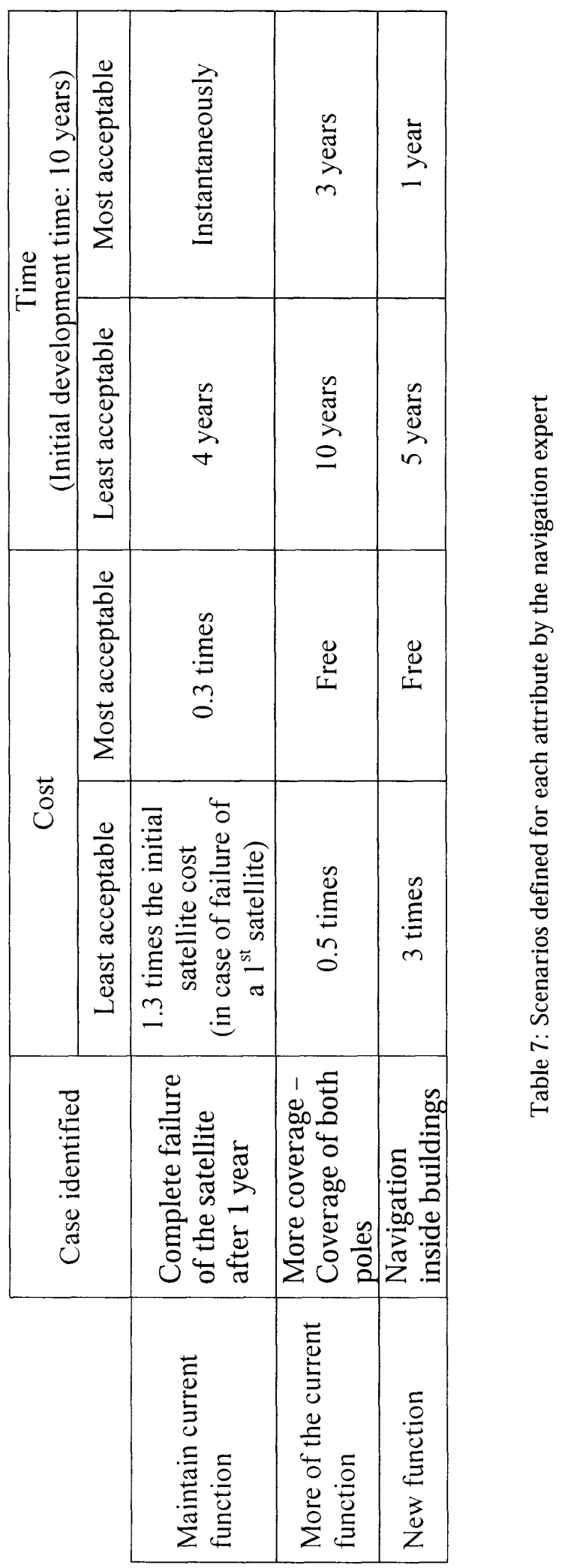




\section{C.3.2.2 Single-attribute utility functions}

During the utility interview, the navigation expert defined first the single-attribute functions presented below.

Figure 18 shows the utility associated with the attribute of maintainability. It presents the relationship between utility and cost to maintain the navigation satellite. The interviewee's utility decreases with the cost to maintain, from 1 when it is free to 0 when it costs $30 \%$ more than the initial satellite cost or more. This relationship is not linear, as opposed to the ones defined by the communications expert, which means that the interviewee is risk-averse, and not risk-neutral.

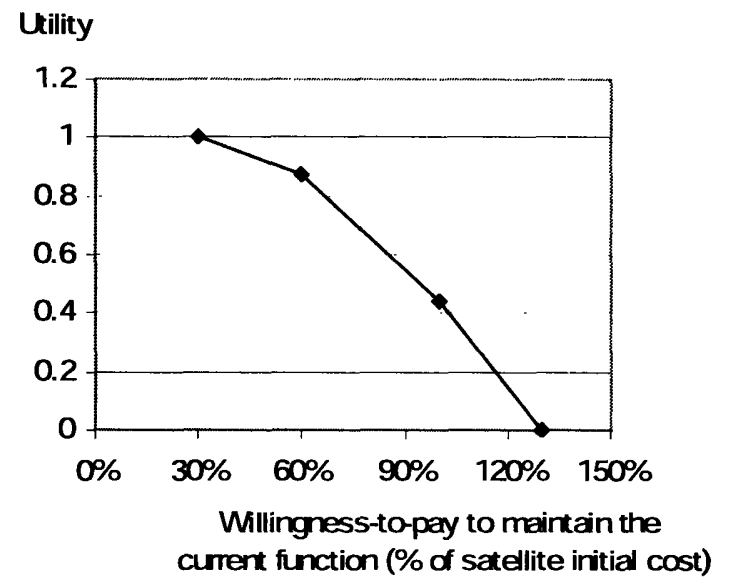

Figure 18: Utility versus cost to maintain the satellite in case of complete failure after 1 year

Figure 19 shows the utility associated with the responsiveness corresponding to maintainability. It presents the relationship between utility and time to maintain the navigation satellite. The interviewee's utility decreases with the time to maintain, from 1 when it is instantaneous to 0 when it takes more than 4 years.

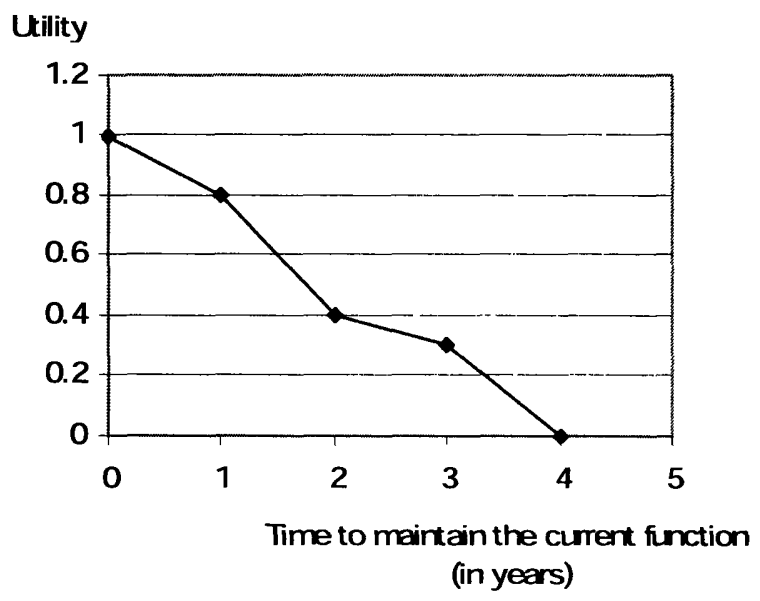

Figure 19: Utility versus time to maintain the satellite in case of complete failure after 1 year 
Figure 20 shows the utility associated with the attribute of scalability. It presents the relationship between utility and cost to get additional coverage. The interviewee's utility decreases with the cost to get additional coverage, from 1 when it is free to 0 when it costs $50 \%$ more than the initial satellite cost or more.

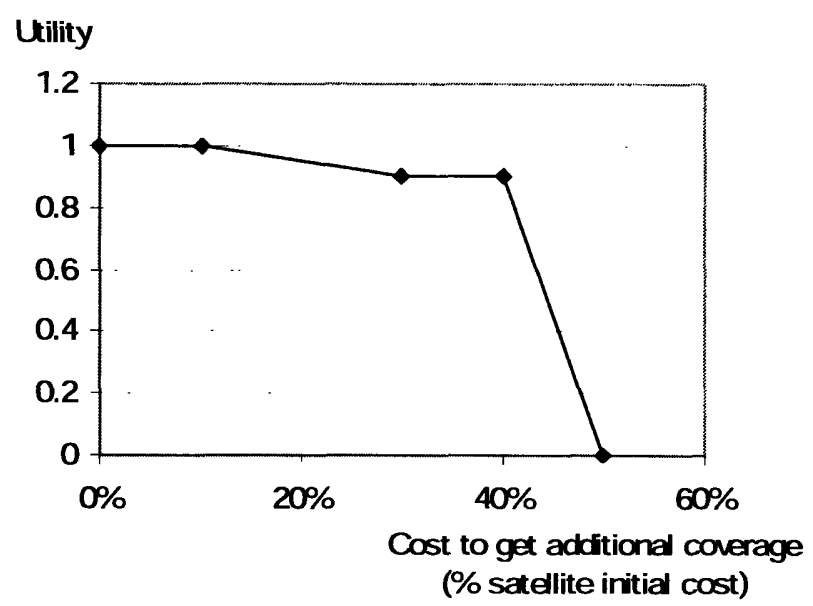

Figure 20: Utility versus cost to get coverage of both poles

Figure 21 shows the utility associated with the responsiveness corresponding to scalability. It presents the relationship between utility and time to get additional coverage. The interviewee's utility decreases with the time to get this additional coverage, from 1 when it is instantaneous to 0 when it takes more than 10 years.

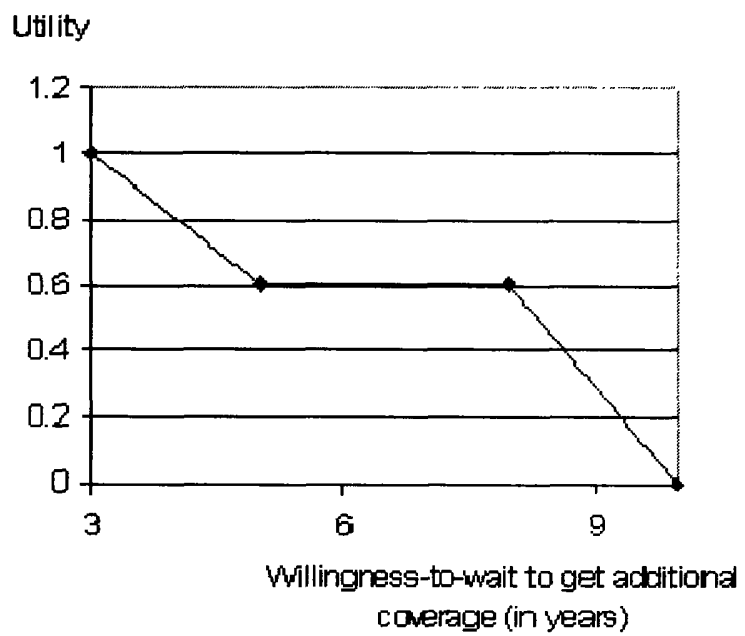

Figure 21: Utility versus time to get coverage of both poles

Figure 22 shows the utility associated with the attribute of flexibility. It presents the relationship between utility and cost to get the possibility of navigating inside buildings. The interviewee's utility decreases with the cost to get additional coverage, from 1 when it is free to 0 when it costs 3 times more than the initial satellite cost or more. 


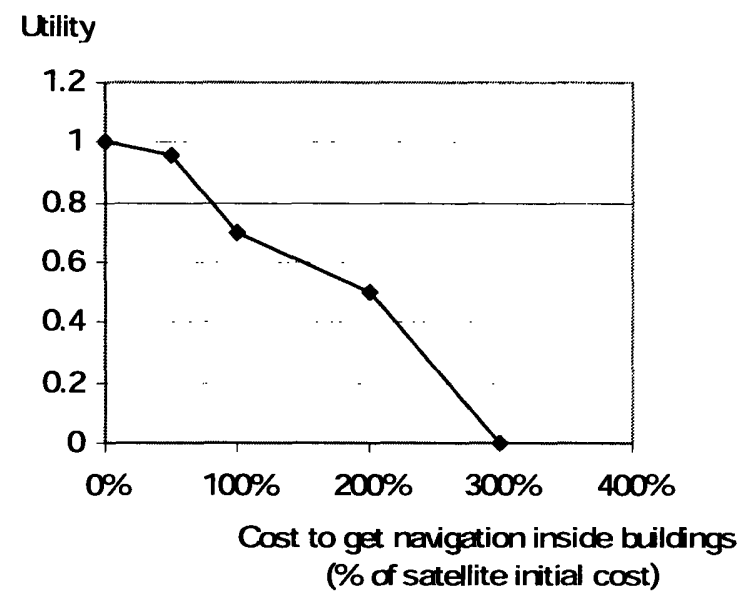

Figure 22: Utility versus cost to get navigation inside buildings

Figure 23 shows the utility associated with the responsiveness corresponding to flexibility. It presents the relationship between utility and time to get the possibility of navigating inside buildings. The interviewee's utility decreases with the time to get this additional coverage, from 1 when it is instantaneous to 0 when it takes more than 5 years.

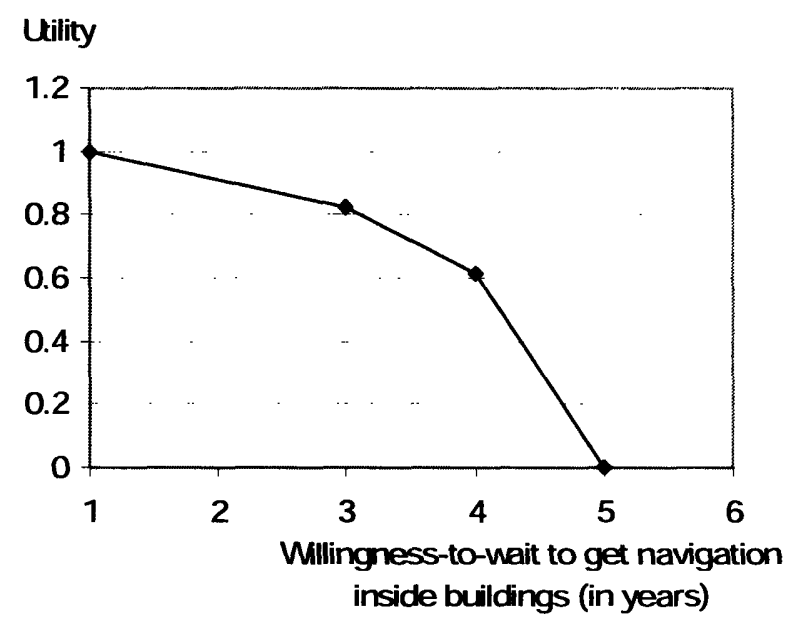

Figure 23: Utility versus time to get navigation inside buildings

\section{C.3.2.3 Scaling factors}

In addition to the single-attribute utility functions, the scaling factor for each attribute is measured during the utility interview. Table 5 presents the value of these different scaling factors. 


\begin{tabular}{|l|l|c|}
\hline \multicolumn{1}{|c|}{ Attributes } & \multicolumn{1}{|c|}{ Corresponding scenario } & $\begin{array}{c}\text { Weighting } \\
\text { parameter in the } \\
\text { utility equation } \\
\text { (in \%) }\end{array}$ \\
\hline Maintainability & Maintaining cheaply & 85 \\
\hline Responsiveness & Maintaining quickly & 40 \\
\hline Scalability & Poles coverage cheaply & 55 \\
\hline Responsiveness & Poles coverage quickly & 70 \\
\hline Flexibility & Navigation inside buildings cheaply & 47.5 \\
\hline Responsiveness & Navigation inside buildings quickly & 25 \\
\hline
\end{tabular}

Table 8: Attributes scaling factors

Table 9 presents the ranking of the attributes by order of importance to the interviewee according to the scaling factors he defined during the utility interview. This ranking is consistent with the preferences expressed by the interviewee during the preliminary questionnaire. His perception of the decision-makers preferences is that the most valued attribute is the possibility of maintaining the existing system, and that cost is more important than time. However, the ranking of the possibilities of getting additional coverage may not be entirely coherent as these possibilities were considered as the least important by the interviewee during the preliminary questionnaire.

\begin{tabular}{|c|c|}
\hline $\begin{array}{c}\text { From the most important (1) } \\
\text { to the least important (6) }\end{array}$ & Scenario \\
\hline 1 & Maintaining cheaply \\
\hline 2 & Additional coverage quickly \\
\hline 3 & Additional coverage cheaply \\
\hline 4 & $\begin{array}{c}\text { Navigation inside buildings } \\
\text { cheaply }\end{array}$ \\
\hline 5 & Maintaining quickly \\
\hline 6 & $\begin{array}{c}\text { Navigation inside buildings } \\
\text { quickly }\end{array}$ \\
\hline
\end{tabular}

Table 9: Attributes ranking based on the scaling factors

C.3.2.4 Multi-attribute utility function

The multi-attribute function that represents the utility as a function of the single-utility functions is:

$$
\begin{array}{r}
\mathrm{U}(\underline{\mathrm{X}})=\frac{1}{-0.9948}\left(\left(-0.8456 * \mathrm{U}_{\text {Maint. }}+1\right)\left(-0.6964 * \mathrm{U}_{\text {Resp.Maint. }}+1\right)\left(-0.3979 * \mathrm{U}_{\text {Scal. }}+1\right)\left(-0.4675 * \mathrm{U}_{\text {Resp.Scal. }}+1\right)\right. \\
\left.\left(-0.5471^{*} \mathrm{U}_{\text {Flex. }}+1\right)\left(-0.2487 * \mathrm{U}_{\text {Resp.Flex. }}+1\right)-1\right)
\end{array}
$$

\section{C.3.3 Conclusions}

Conclusions for each field of application can be drawn from these utility interviews. First, communications satellites users would value scalability and flexibility and their 
corresponding forms of responsiveness but not maintainability as defined in this study. Scalability is the most valued attribute of all, and cost is more important than time in decisions to maintain or upgrade a system. On the other hand, navigation satellites users would value all of these attributes. Maintainability is the most valued one, and cost is more important than time in decisions to maintain or upgrade a system.

Conclusions can also be drawn by comparison of both fields of application. First, concerning maintainability, both of these applications are based on constellations so that the dynamics, especially in case of unanticipated failure, is different than for singlesystem-based applications. In case of failure, a communications satellite is replaced by another one already on-orbit, and the failure of a navigation satellite is more or less critical, depending on the number of other failures in the constellation and on the time to the next scheduled launch, as new satellite are regularly launched. Second, concerning scalability, these two applications gives two extreme examples of what scalability can mean. There is the possibility of incrementally adding for instance transponders on communications satellites, but to get a same function but additional performance navigation users need more satellites. The difference is in the nature of the payload and of the application. This effect has an impact on the importance given to time versus cost by the interviewee. For instance, the navigation expert mentioned that time is not that critical because new satellites are regularly launched.

\section{C.4 Lessons learned}

\section{C.4.1 Method used}

The method used in this study to determine the multi-attribute utility function is based on the lottery equivalent probability (LEP) approach. This approach is difficult to understand for the interviewee and answering the questions is long and demanding for them. In addition, it was difficult for the interviewees to answer questions with six different attributes, as it is very challenging for the interviewees to consider and compare uncertain outcomes with six different dimensions. This was made a little easier by the fact that the six attributes correspond only to three scenarios in this study.

This method constrains the interviewee on how he/she expresses the cost and time metrics. As the several attributes metrics are defined in units of cost and several others in units of time, the value of these attributes must be defined in similar ways for the comparison between costs and times to be possible in the questions used to determine the scaling factors.

As mentioned above, the validation interview was not possible because of the interviewees' availability, but given the number of attributes it would have been even more challenging, and probably of little value because of the large number of attributes.

In these interviews it was very important to explain to the interviewees that what was measured was their perception and not the "truth". Interviewees tend to consider that they are being tested on what they know and do not know, which was clearly not the intent in this study.

Finally, the LEP approach with cost and time parameters seem to lead the interviewees to make expected time and expected cost calculations, rather than expected utility calculations. This was the first reaction of both interviewees to the LEP questions; they answered the first question of the single-attribute function by equating the expected cost 
of the first lottery with the expected cost of the second one. An explanation on what this method means for their utility functions was useful to help them making up their mind on whether expected cost and time calculations was the right approach.

\section{C.4.2 Attributes}

First, the choice of the attributes turned out to be quite exhaustive. No scenario of uncertain change during a satellite lifetime that would not be covered by any of the attributes was mentioned during the interviews. Moreover the attributes seem to have been treated as independent by both interviewees even though they were defined based on common scenarios.

However some limitations of the attribute metrics appeared during the interview.

First, the communications expert mentioned that operational costs and the impact of any change in the space segment on the ground segment should be taken into account. This turned out to be an important issue for communications satellite but not for navigation satellites.

Second, the new function mentioned by both interviewees cannot yet be implemented given today's technology. It is therefore impossible to foresee the cost of these future systems. As the flexibility measurement takes into account a new payload module with the new payload, it was assumed that the new payload would cost the same amount as the current one, as this payload would be sized by the existing supporting functions. This hypothesis is questionable given the interviewees' answers; however it would be completely arbitrary to define the cost of the new payload as 1.2 or 1.5 times the cost of the current one.

\section{C.4.3 Scenarios}

It was difficult for the interviewees to define the scenarios, because of the difficulties for them to match the users' needs with one of the pre-defined "ilities"and their general scenarios. This is one of the challenges of this method when used with "ilities" attributes, as the attributes generally used in this method are traditional performance parameters that are clearer for the interviewees. One of the interviewees mentioned that it would have been helpful to provide him with examples of scenarios, but it is then a trade-off between helping the interviewee and providing him with information that may influence his answers.

Second, the interviewee must be explicitly asked to define scenarios based on the space segment value proposition and not on the entire system value proposition. The interviewees tended to have a system approach of their systems, but what was of value to this study was only the change in space segment so that the scenarios should focus on its value.

Third, it turned out to be very important in the definition of the maintainability scenario to make the distinction between degradation (which can be foreseen and/or monitored) and unanticipated failure. It was also very important to discuss the time factor, i.e. the impact on their answers of the time when the failure occurs.

\section{C.4.4 Implementation of the method}

First of all, the three-step approach has shown many advantages. Because of the nature of the attributes, a preliminary questionnaire was necessary to make sure the interviewee 
understands the attributes and to have him/her define the scenarios. It was also used as a validation of the result of the utility interview. It was beneficial to have two separate meetings to leave some time for the interviewees to digest the attributes and their scenarios. It also allows the interviewer to adapt the questions to the scenarios defined by the interviewee.

Second, the format of the LEP questions is difficult to understand, especially with six attributes. The visualization method developed turned out to be very helpful.

The main difficulties met by the interviewees were first to understand the attributes investigated, and second to understand the format of the utility questions. The preliminary questionnaire helped with the first difficulty, the visualization of the questions helped with the second.

One of the lessons learned is the need to make it clear for the interviewee who he/she should answer as. In the preliminary questionnaire, it was confusing for the interviewees to be asked to answer some questions as a user, as a decision-maker, or as a designer.

Finally, the general method used for the utility interviews should be applied to many more interviewees to make the results truly representative of users community.

\section{C.4.5 Tools}

If more interviews are made, it may be worth developing a simulation tool, based on MS Excel or Matlab, which would automatically change the probabilities in the questions according to the interviewee's answers and plot the utility functions in real time to check the "shape" of the functions and make sure the interviewee's answers are coherent.

\section{Analysis at System Level}

This chapter presents the main results of the architectural analysis at spacecraft level. Different missions and scenarios are used to illustrate the impact of fractionation on the spacecraft mass, cost, attributes, and utility. In each example, the different fractionated architectures introduced in Section B are compared with an equivalent traditional one.

\section{D.1 Mass}

One of the expected drawbacks of the spacecraft fractionation is a mass penalty. The mass model quantifies this penalty for all the fractionation strategies. Figure 24 presents the mass penalty as a percentage of the traditional spacecraft mass for each of the fractionated architectures applied to three types of space missions. Figure 24a) and b) correspond to the two power subsystem fractionation strategies discussed in Section B3. As expected, the mass tends to increase with the fractionation level along the $\mathrm{x}$ axis. There is a clear difference in mass penalty depending on the mission. The navigation mission has the largest mass penalty, which varies between $220 \%$ and $300 \%$, whereas the sensing mission has the smallest one, which varies between $100 \%$ and $140 \%$. Again, in this model the variation in mass penalty among mission types is due to the modeled differences in subsystem mass ratios based on historical tendencies for these three types of space missions. A remarkable feature is the step up in mass penalty that occurs at the fractionation of the power subsystem, which is due in case a) to the additional hardware 
required to transmit and receive power, and in case b), in addition to this hardware, to the complete fractionation of the power generation function into the power module. The only differences between the two power subsystem fractionation strategies occur in the continuous fractionation of the power subsystem, i.e. in the middle third of Figure 24a) and $b$ ).

As mentioned above, the mass tends to increase with the fractionation level. One can notice that in some cases the total system mass decreases with the fractionation of the attitude control and propulsion subsystems in the final third of Figure 24a) and b). When this fractionation occurs, one or two new modules are created, but the attitude control and propulsion functions are taken out of all the other modules and centralized. The difference between those two effects, which are opposite in terms of mass impact, leads in those cases to a decrease in the total system mass; the mass decrease resulting from the concentration of those functions is larger that the mass increase due to the addition of new modules.
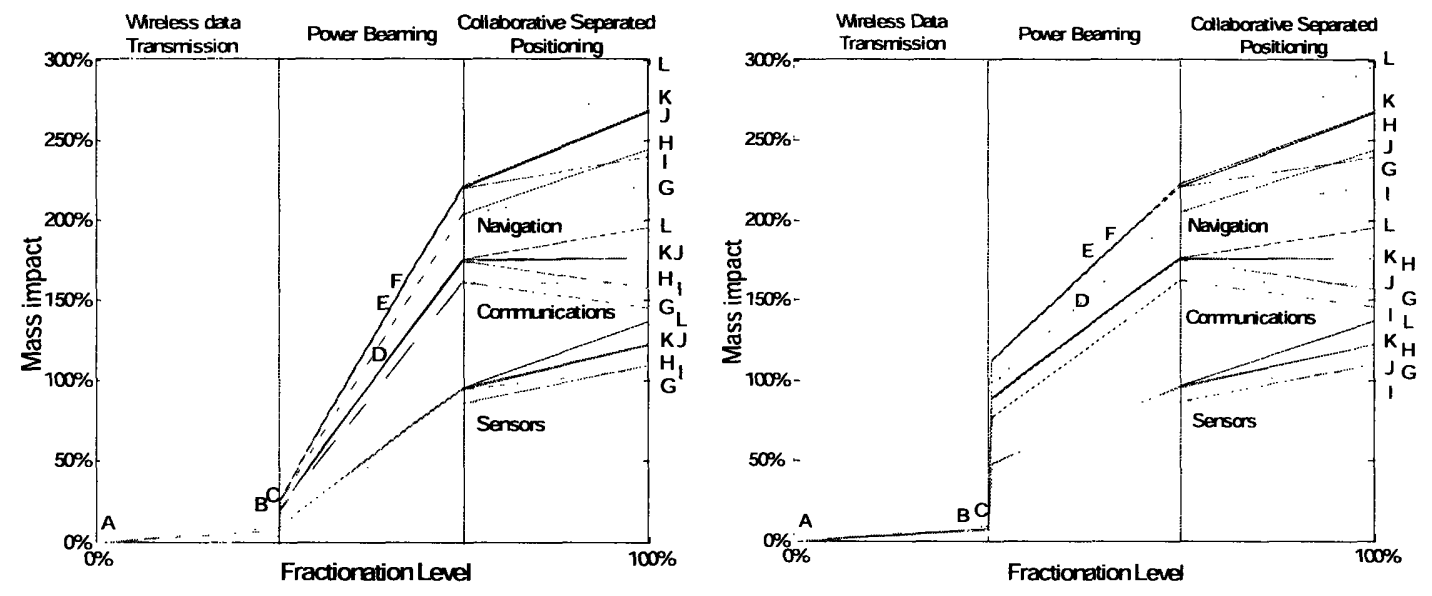

Figure 24: Mass penalty due to fractionation for different fractionated architectures

\section{2 Cost}

Another expected drawback of the spacecraft fractionation, linked to the mass penalty, is a cost penalty. The cost model quantifies this penalty for all the fractionation strategies. In this section, the costs of the fractionated and traditional architectures are presented for two different missions. The first example is a communications mission, and the second example is a navigation mission.

\section{D.2.1 Communications missions}

The first example used to illustrate the impact of fractionation on the spacecraft mass is a communications satellite with a total mass of $2,000 \mathrm{~kg}$ with 24 transponders (36 MHz) with a "standard" design.

Figure 25 presents the cost penalty as a percentage of the traditional spacecraft cost for all the fractionated architectures. This figure corresponds to the power subsystem fractionation strategy in which power generation and storage are simultaneously fractionated. The costs presented include the development, manufacturing, integration, 
and launch costs for both the payload and the bus. The development effort was assumed to be $10 \%$ of a full development effort because of the standard design, as advised in (Wertz and Larson, 1999).

The cost penalty varies from $10 \%$ for Architecture B to $120 \%$ for architecture L. One can notice that the cost always increases with the fractionation level along the $\mathrm{x}$ axis. This is in contrast to the mass penalty graphs given in Figure 24a) and b), which for some architectures show a decrease in mass in the final stage of fractionating the attitude control and propulsion subsystems.

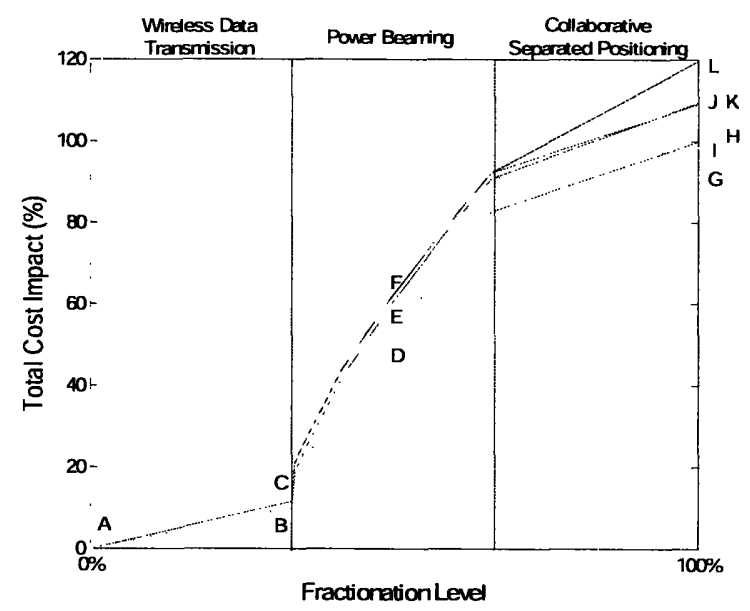

Figure 25: Impact of fractionation on the communications satellite initial cost for different architectures

These figures significantly vary with the size of the payload. The cost impact decreases when the payload size increases. This phenomenon is due to the fact that the cost of the payload increases more than the cost of the corresponding supporting functions with the payload size. Therefore the cost of the payload relative to the supporting functions becomes increases and the impact of fractionation on the supporting functions decreases. In this respect, it is interesting to compare this Figure 25 with Figure 26 which removes the influence of the payload on the cost impact. Figure 26 shows for the same example the cost penalty on the supporting functions only, i.e. the ratio between the fractionated architectures cost, if the payload cost is excluded, and the traditional architecture cost, also if the payload cost is excluded. It is clear that the relative cost impacts are much larger than the ones presented on Figure 25 . The relative costs vary between $25 \%$ for Architecture B and $300 \%$ for Architecture L. 


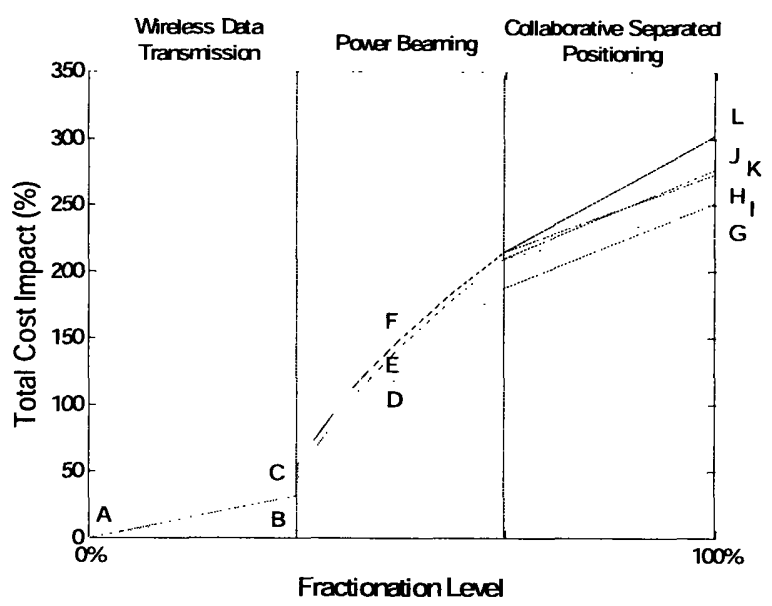

Figure 26: Impact of fractionation on the initial cost of the supporting functions for different architectures

\section{D.2.2 Navigation missions}

The second example used to illustrate the impact of fractionation on the spacecraft mass is a navigation satellite based on Navstar GPS Block IIA satellites.

Figure 27 presents the cost penalty as a percentage of the traditional spacecraft cost for all the fractionated architectures. This figure also corresponds to the power subsystem fractionation strategy in which power generation and storage are simultaneously fractionated. The costs presented include the development, manufacturing, integration, and launch costs for both the payload and the bus. The development effort was assumed to be $50 \%$ of a full development effort because of commonalities with previous navigation satellites, as advised in (Wertz and Larson, 1999).

The cost penalty varies from $10 \%$ for Architecture B to $145 \%$ for architecture L. One can notice that the cost always increases with the fractionation level along the $\mathrm{x}$ axis.

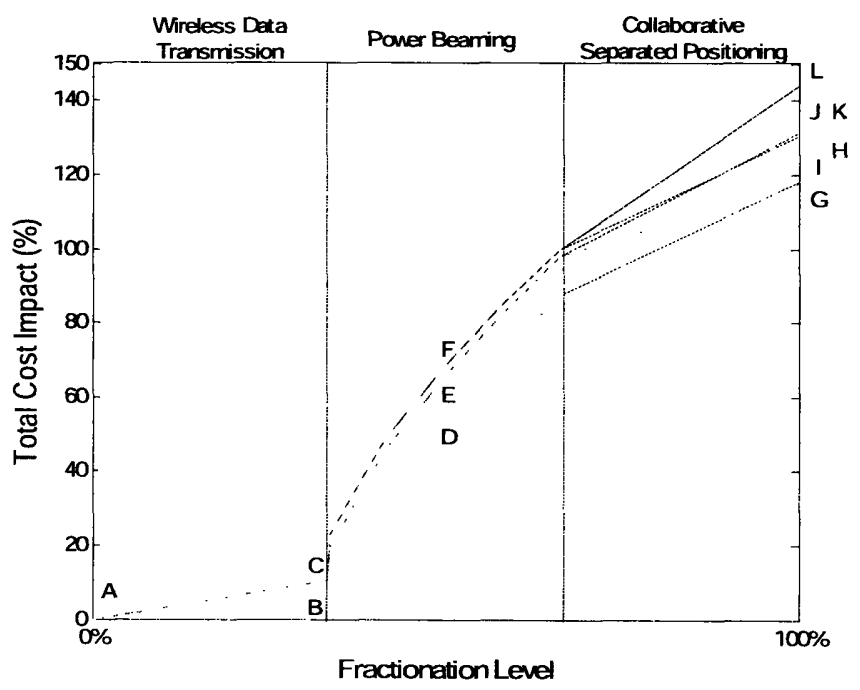

Figure 27: Impact of fractionation on the navigation satellite initial cost for different architectures 


\section{D.3 Attributes}

The communications mission introduced in the previous section is used to illustrate the measurement of the different attributes.

\section{D.3.1 Maintainability}

Two examples for the same communications mission are presented to illustrate the maintainability metric.

Again in these examples, the power fractionation strategy in which both the power generation and storage functions are fractionated simultaneously is the one assumed.

The first example assumes a complete failure of the communications subsystem. If the spacecraft has a fractionated architecture, only the module that contains the communications subsystem has to be exchanged, whereas if it has a traditional architecture, a whole new spacecraft is assumed to be needed.

Figure 28 presents the cost ratio between the module that contains the communications subsystem and the traditional spacecraft. It is a ratio between recurring costs, as the module is assumed to be exchanged with an identical one. This ratio is expected to get smaller as the fractionation level increases. In fact, the ratio does get smaller for the architectures in which the communications subsystem is not located in the same module as the power subsystem, i.e. architectures I through L. For architectures $\mathrm{K}$ and L, the cost ratio becomes as small as $7 \%$. If the communications subsystem is in the same module as the power subsystem, as in architectures $\mathrm{G}$ and $\mathrm{H}$, this module becomes more expensive than the traditional spacecraft itself as the fractionation level increases. This result highlights the important and often driving role played by the power subsystem in fractionated architectures.

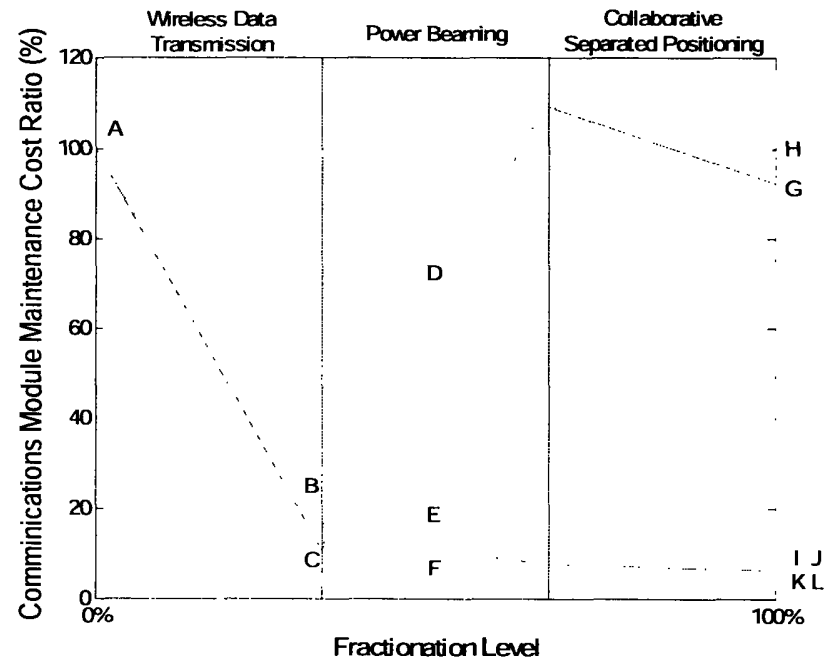

Figure 28: Cost of the communications module exchange relative to the traditional spacecraft cost (communications mission) 
The second example looks closer at the particular case of the power module. It assumes a complete failure of the power subsystem. In the same way as in the first example, if the spacecraft has a fractionated architecture, only the module that contains the power subsystem has to be exchanged, whereas if it has a traditional architecture, a whole new spacecraft is assumed to be needed.

Figure 29 presents the cost ratio between the module that contains the power subsystem and the traditional spacecraft. On the left third of the figure, the module that contains the power subsystem is the payload module. Then, as the fractionation level increases, the power fractionated is either, like in architecture $\mathrm{D}$, added to the communications and data handling module, or a new power module is created, like in architectures $\mathrm{E}$ and $\mathrm{F}$. This difference explains the step when the power starts getting fractionated in the case of architecture D.

The major impact of the power subsystem on the fractionation results becomes obvious in Figure 29. For all the architectures, as the power fractionation increases, the power module becomes more expensive than the initial traditional spacecraft. This means that with the traditional spacecraft cost relationships, fractionation would not make the spacecraft more maintainable in case of failure of the power subsystem.

An interesting feature is that the architectures in which the power subsystem is not fractionated in its own module, i.e. architectures $\mathrm{G}$ and $\mathrm{H}$, are not the ones with the most expensive power module. Again, this illustrates two opposite effects. When the power subsystem gets fractionated in its own module, as opposed to when it gets fractionated in the communications and data handling module, the power module does not contain any other subsystem, so it should be less expensive, but it has to generate extra power to beam power to the communications and data handling module(s), which makes it more expensive.

This second example illustrates that when considering technology development to improve fractionated spacecraft design the main focus should be on power subsystems.

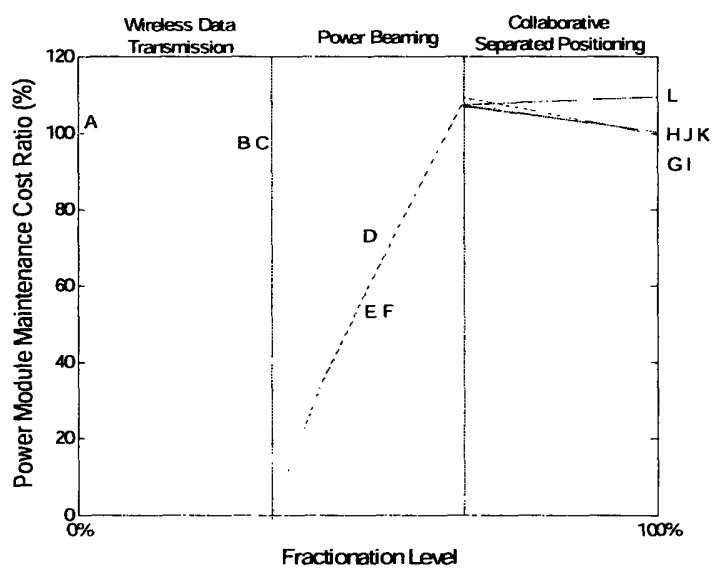

Figure 29: Cost of the power module exchange relative to the traditional spacecraft cost (communications mission)

\section{D.3.2 Scalability}

The scenario chosen to assess the scalability of the architectures focuses on the payload. The example presented to illustrate the scalability metric assumes the need for a new 
higher-performance payload that would be $\mathrm{p} \%$ heavier than the initial one and that would require $\mathrm{p} \%$ more power, with $\mathrm{p}<1$. If the spacecraft has a fractionated architecture, an additional scaled-down payload module has to be added, but if it has a traditional architecture, a whole new spacecraft is assumed to be needed.

Figure 30 shows the cost ratio between the additional scaled-down payload module and the corresponding scaled-up traditional spacecraft. There is only one curve for each value of the percentage of performance increase desired, $p$, since only the payload module is being altered to achieve scalability, and not the infrastructure modules. The costs include a limited development effort to scale-up the system as well as recurring costs. For the more fractionated architectures, and a performance increase of $p=50 \%$, the payload module addition costs less than $30 \%$ of the traditional spacecraft cost, which corresponds to significantly improved scalability for fractionated spacecraft over traditional spacecraft.

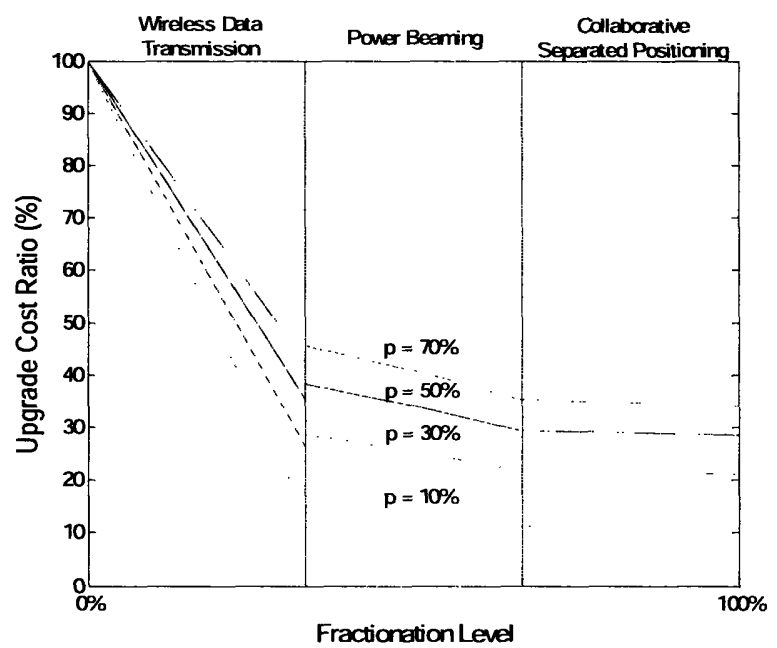

Figure 30: Cost of the additional payload module relative to the scaled-up traditional spacecraft cost for different percentages of performance increase $p$ (communications mission) Note: Each line represents all architectures

\section{D.3.3 Flexibility}

As in the scalability assessment, the scenario chosen to assess the flexibility of the architectures focuses on the payload. The example presented to illustrate the flexibility metric assumes the need for a new function, which means a new payload. It is assumed that the new payload requires a new development effort. Again, if the spacecraft has a fractionated architecture, only the payload module has to be exchanged, but if it has a traditional architecture, a whole new spacecraft is assumed to be needed.

Figure 31 shows the cost ratio between the newly developed payload module and the corresponding traditional spacecraft. The costs include a complete new development effort as well as recurring costs. Again, as only the payload module is exchanged, there is only one curve for all the architectures. For the more fractionated architectures, a payload module exchange costs less than $65 \%$ of the whole traditional spacecraft exchange cost, which corresponds to significantly improved flexibility for fractionated spacecraft. 


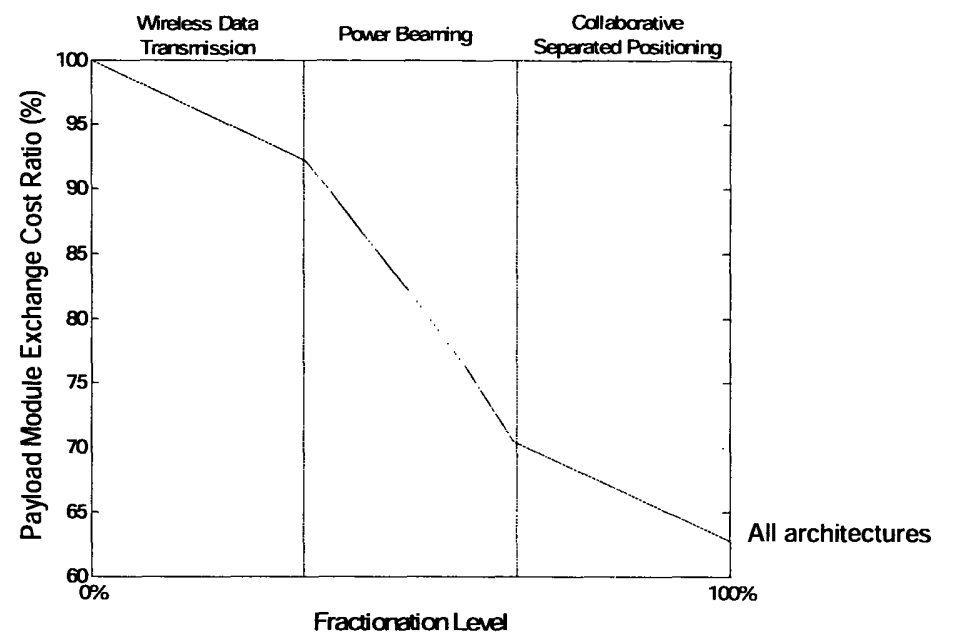

Figure 31: Cost of the new payload module relative to the traditional spacecraft cost (communications mission)

\section{D.3.4 Responsiveness associated with maintainability}

The scenario chosen to assess the architecture responsiveness associated with maintainability is the same as the one used for maintainability.

Figure 32 shows the ratio between the time necessary to manufacture, integrate, assemble and test a new module and the time necessary for a whole traditional spacecraft.

As shown in Figure 29, in case of power subsystem failure, once the power subsystem is fractionated, the more fractionated the architecture is, the longer the time necessary to maintain it. Moreover the module exchange for the most fractionated architectures takes longer than for the traditional architecture. It is noteworthy that this result, and the following ones, is based on the hypothesis that the fractionated spacecraft will have comparable development, manufacturing, integration, and test cycles as a traditional spacecraft, which is unfavorable to the fractionation concept even when looking only at the payload module. 


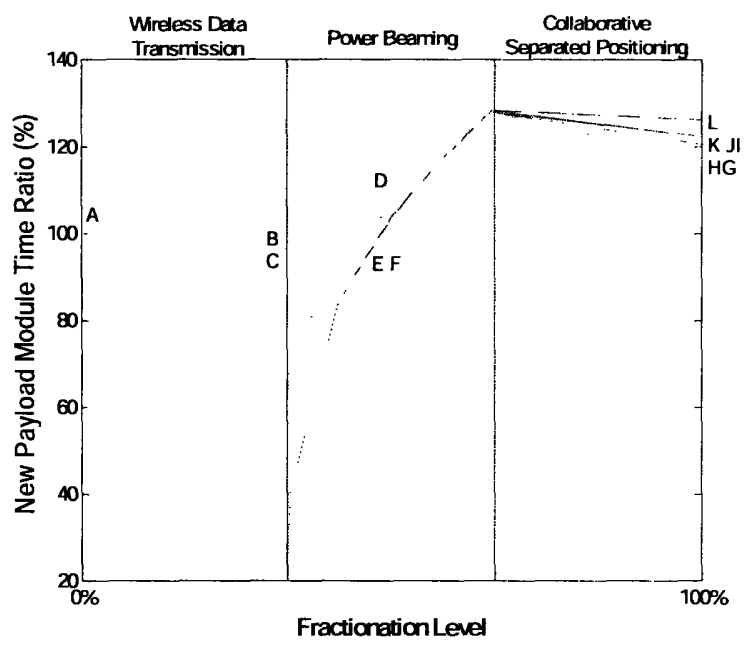

Figure 32: Time necessary to exchange the power module relative to the traditional spacecraft (communications mission)

D.3.5 Responsiveness associated with scalability

The scenario chosen to assess the architecture responsiveness associated with scalability is the same as the one used for scalability.

Figure 33 shows the ratio between the time necessary to develop, manufacture, integrate, assemble and test an additional module and the time necessary for a whole scaled-up traditional spacecraft. This ratio gets lower than $50 \%$ for a $10 \%$ increase in the performance level, and is still lower than $70 \%$ for an increase of $70 \%$. The relative gain in time is clearly less than proportional to the increase, $p$.

The time necessary to get the increased performance level generally decreases with fractionation, in a similar way for different value of performance increase, p. There is again a step when the power is fractionated, as already seen above, so that the less fractionated Architectures D, E, and F are less "responsive" than Architectures B and C. 


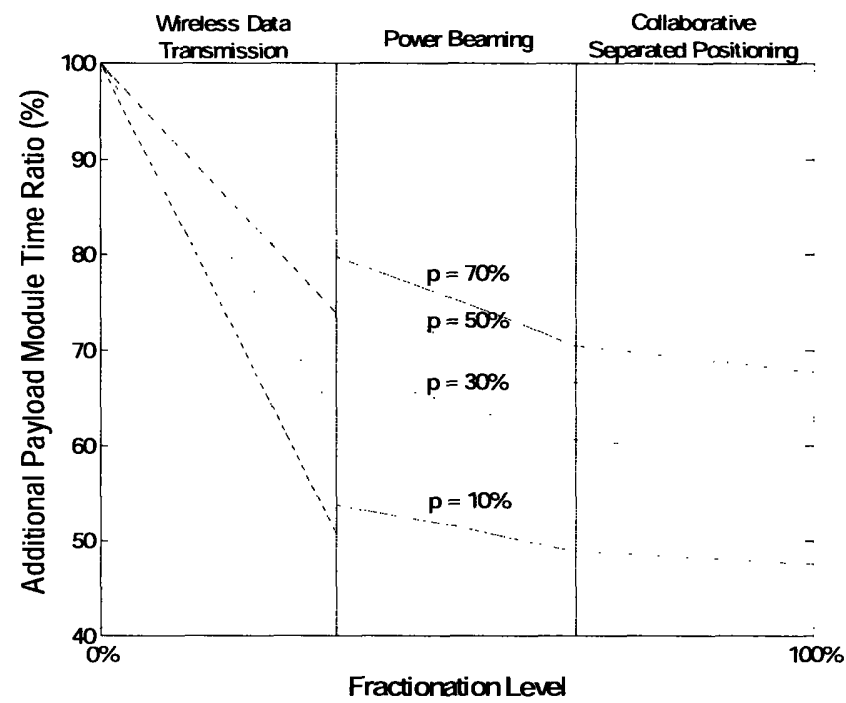

Figure 33: Time necessary for the additional payload module relative to the scaled-up traditional spacecraft for different percentages of performance increase $p$ (communications mission) Note: Each line represents all architectures

\section{D.3.6 Responsiveness associated with flexibility}

The scenario chosen to assess the architecture responsiveness associated with flexibility is the same as the one used for flexibility.

Figure 34 shows the ratio between the time necessary to develop, manufacture, integrate, assemble and test a new payload module and the time necessary for a whole traditional spacecraft. This ratio gets as low as $70 \%$ for the most fractionated architectures. In this scenario compared for instance to the scalability one, the payload, which is newly developed, becomes more important relative to the rest of the payload module, so that the gain in time is more limited.

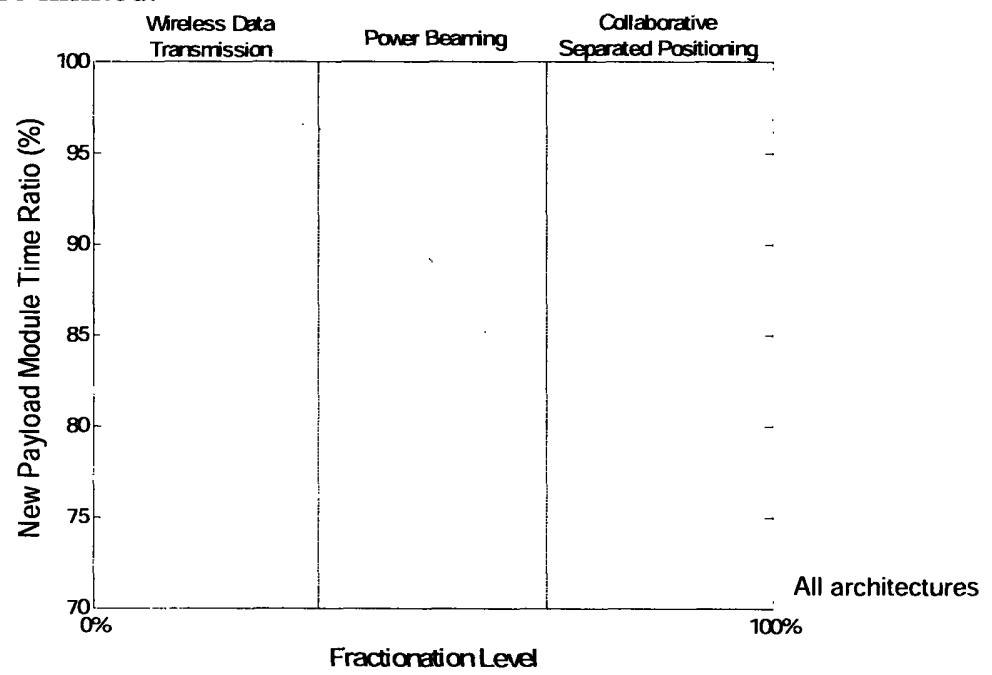

Figure 34: Time necessary for a new payload module relative to the traditional spacecraft (communications mission) 


\section{D.4 Utility}

Different utility figures based on the communications and navigation missions introduced in Chapter B are presented below. The attributes are presented for the communications mission except for the two attributes of maintainability and its corresponding form of responsiveness for which the navigation mission is used, as the communications expert excluded these attributes. These utility figures for each attribute are based on the singleutility functions as defined by the communications expert.

One should keep in mind that all the figures presented above were relative figures, i.e. values for the fractionated architectures relative to the value for the equivalent traditional architecture, whereas the ones that follow are absolute figures.

\section{D.4.1 Maintainability}

Figure 35 shows the utility associated with maintainability of the different architectures. This figure corresponds to the failure of the power subsystem. One should bear in mind that these results are based on the assumption that if the power subsystem is partly fractionated, the part of the system that is assumed to fail is the one located in the module containing the power subsystem.

The utility of the traditional architecture is 0.46 and the utility of the fractionated architectures varies between 0 and 1 . As presented on Figure 29 for the communications mission, the cost of maintaining the module containing the power subsystem initially decreases with fractionation, but as the fractionation of the power subsystem increases this cost increases and gets higher than the cost of maintaining a traditional spacecraft. These trends are similar for navigation missions. Thus the utility associated with the maintainability of the spacecraft for the power subsystem increases from the traditional architecture to architecture B and C. It also increases when the power starts getting fractionated but then decreases with the fractionation of the power subsystem to finally reach 0 when all the power subsystem is fractionated.

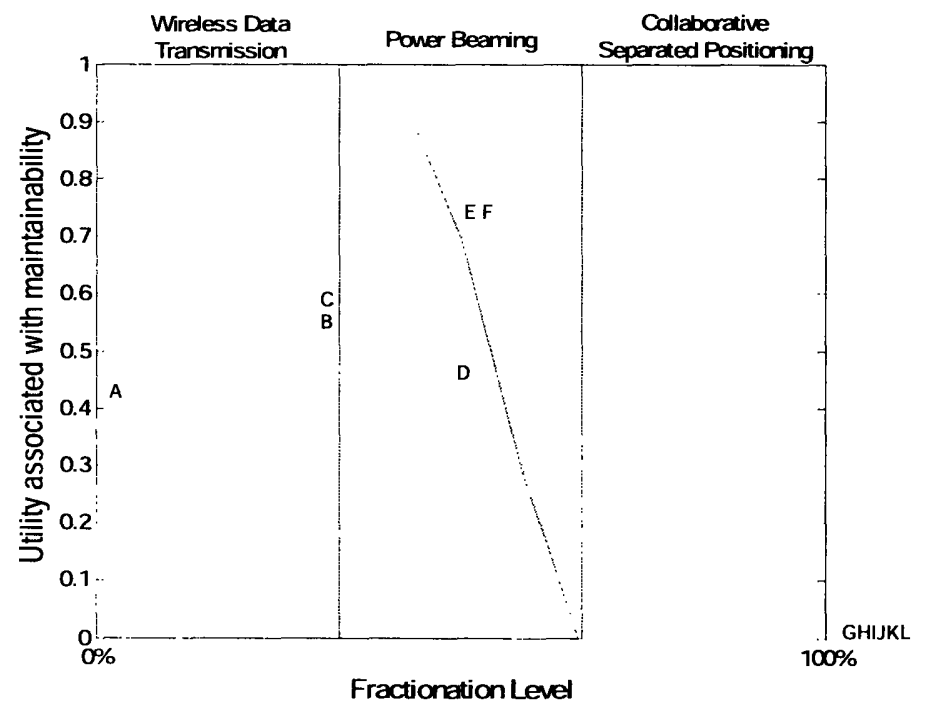

Figure 35: Utility associated with maintainability (navigation mission) 


\section{D.4.2 Scalability}

Figure 36 shows the utility associated with scalability of the different architectures for a percentage of performance increase $p$ of $50 \%$. For any given $p$, there is only one line for all the architectures as only the payload module, which is similar for the architectures with the same fractionation level, influences the utility value. The utility of the traditional architecture (which is scaled-up by $50 \%$ ) is 0 and the utility of the fractionated architectures varies between 0.6 and 0.7 . As expected the more fractionated the architecture is, the higher the utility.

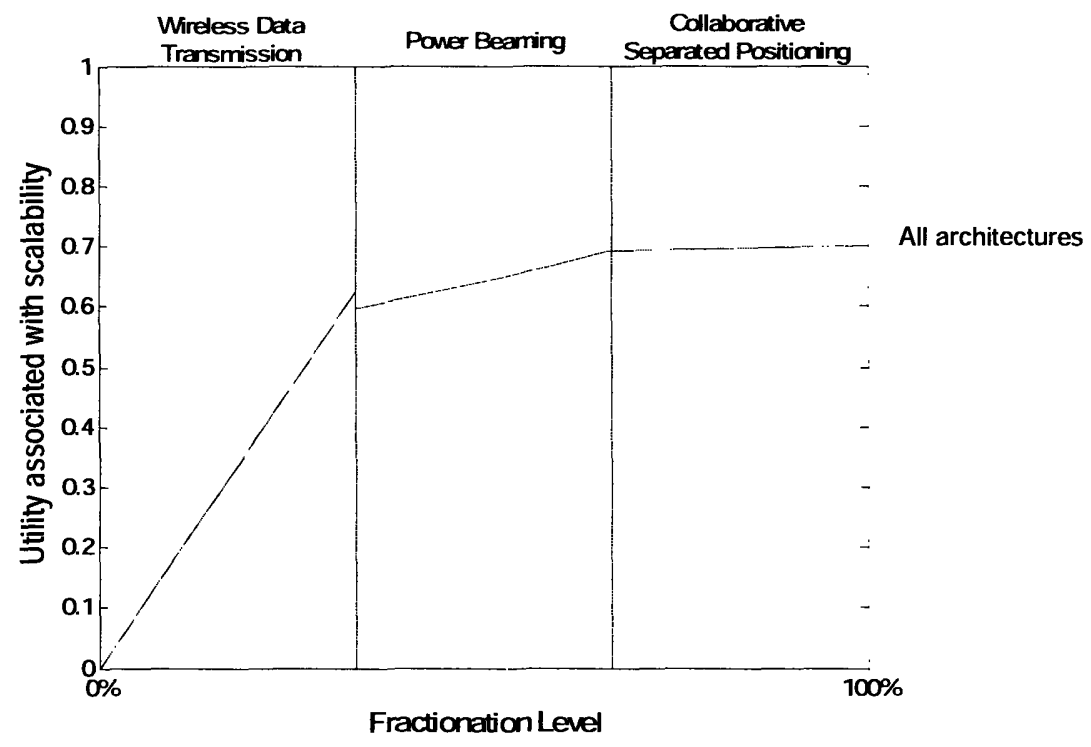

Figure 36: Utility associated with scalability for $\mathrm{p}=50 \%$ (communications mission)

\section{D.4.3 Flexibility}

Figure 37 shows the utility associated with flexibility of the different architectures. Again, there is only one line for all the architectures as only the payload module, which is similar for the architectures with the same fractionation level, influences the utility value. The utility of the traditional architecture is 0.13 and the utility of the fractionated architectures significantly varies between 0.2 and 0.45 . As expected the more fractionated the architecture is, the higher the utility. 


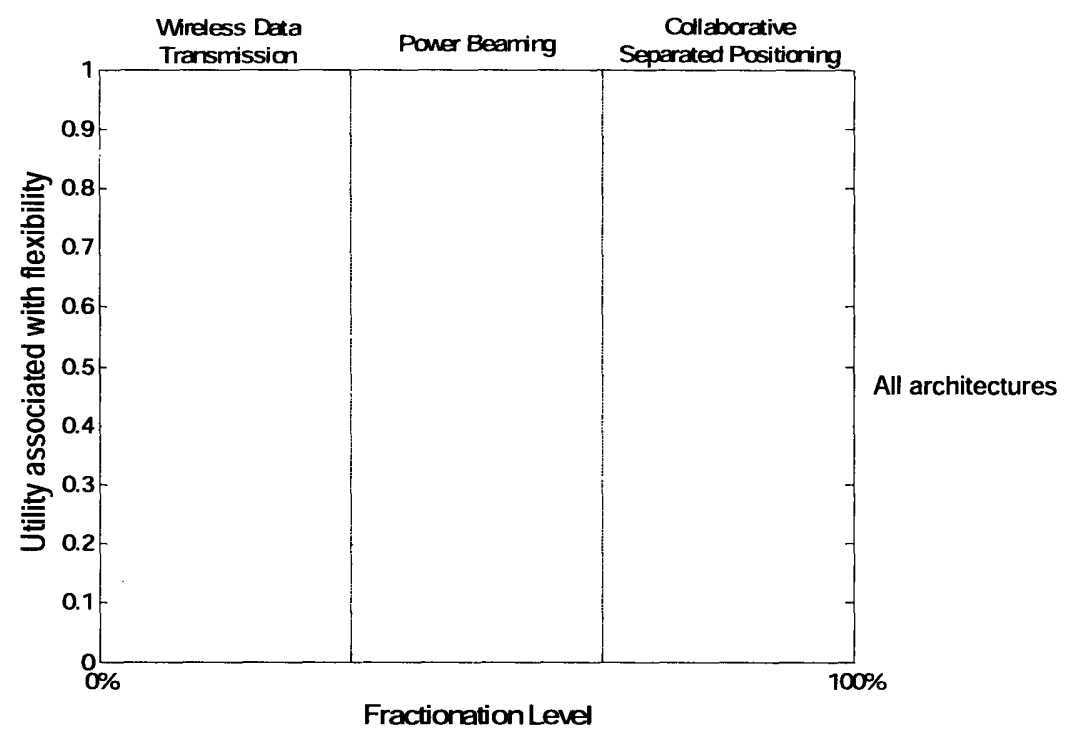

Figure 37: Utility associated with flexibility (communications mission)

D.4.4 Responsiveness associated with maintainability

Figure 38 shows the utility associated with responsiveness of the different architectures. This figure corresponds to the failure of the power subsystem. The utility of the traditional architecture and of most fractionated architectures is 0 , which means that the time to maintain the power subsystem is too long given the navigation users' needs. The utility is greater than 0 only when a couple of percents of the power subsystem is fractionated in the power module.

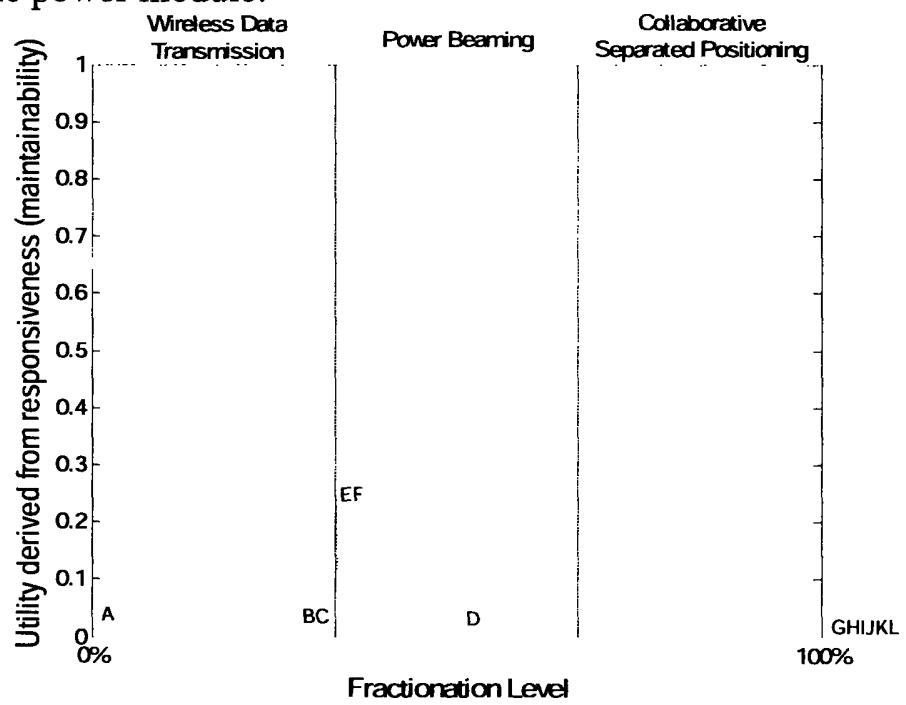

Figure 38: Utility associated with responsiveness (navigation mission)

D.4.5 Responsiveness associated with scalability

Figure 39 shows the utility associated with responsiveness of the different architectures for a percentage of performance increase $\mathrm{p}$ of $50 \%$. The utility of the traditional 
architecture is 0 and the utility of the fractionated architectures varies between 0.22 and 0.33 . As expected the more fractionated the architecture is, the higher the utility.

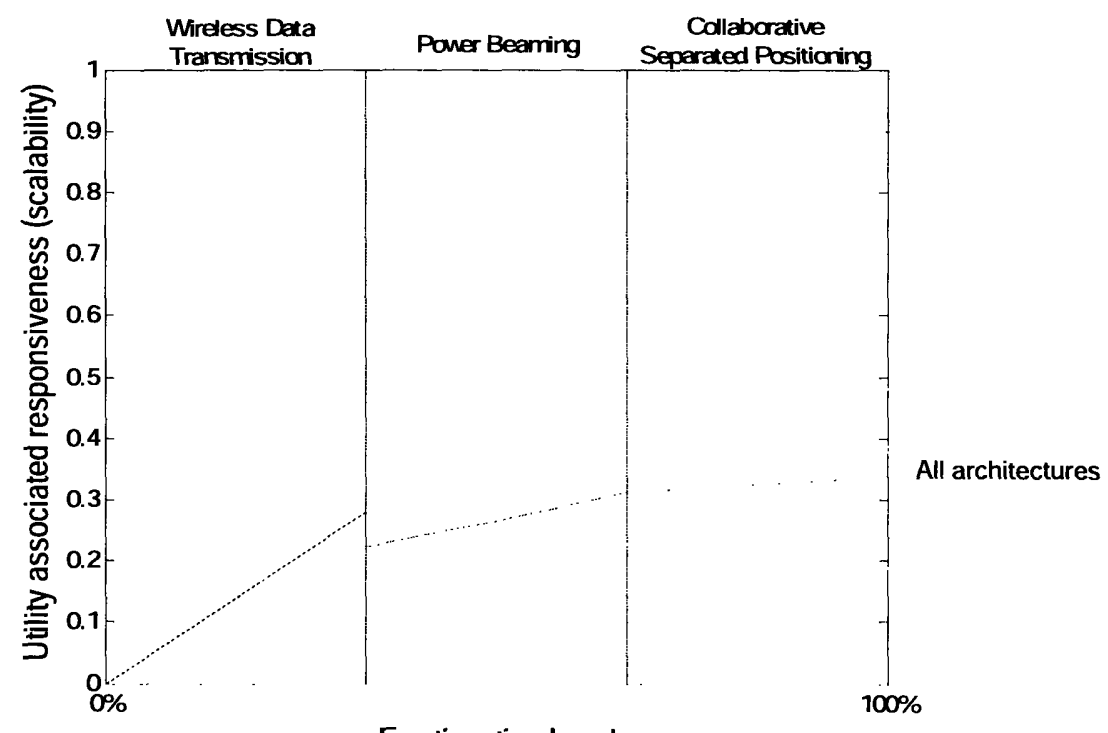

Figure 39: Utility associated with responsiveness for $\mathrm{p}=50 \%$ (communications mission)

\section{D.4.6 Responsiveness associated with flexibility}

Figure 40 shows the utility associated with responsiveness of the different architectures. The utility of the traditional architecture is 0.13 and the utility of the fractionated architectures significantly varies between 0.15 and 0.40 . As expected the more fractionated the architecture is, the higher the utility.

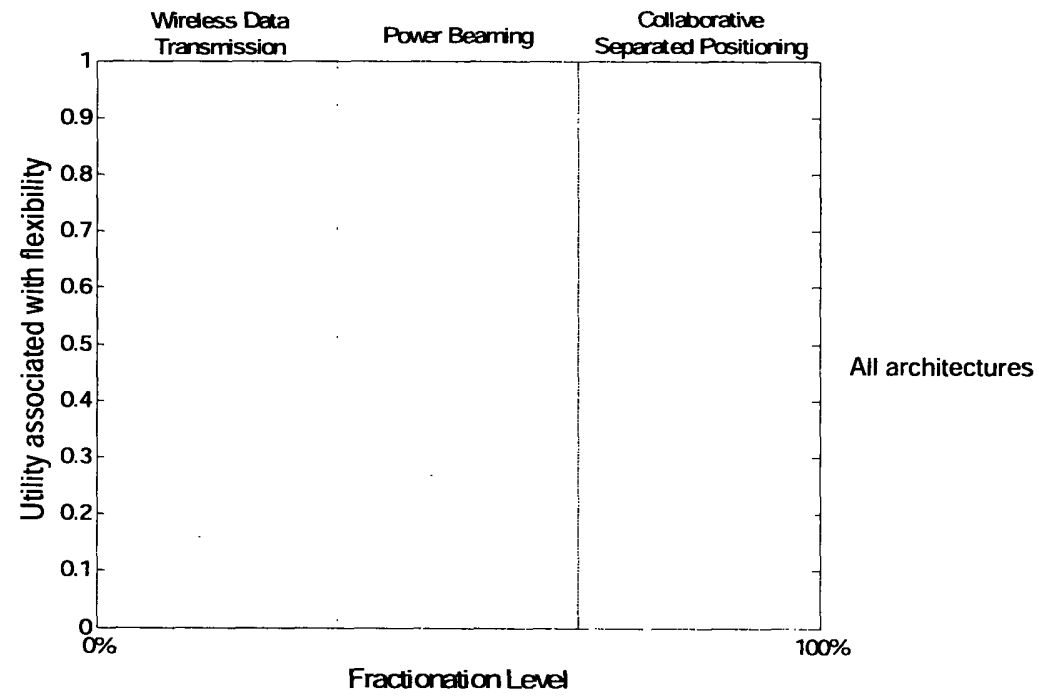

Figure 40: Utility associated with responsiveness (communications mission) 


\section{D.5 Multi-attribute utility}

The utility figures presented in this section are based on the communications and navigation mission examples introduced in Chapter B. These utility figures include all the attributes of interest to the users and are based on multi-attribute utility functions as defined by the communications and navigation experts.

\section{D.5.1 Communications missions}

Figure 41 shows the utility of each architecture, the traditional one and the fractionated ones from A to $\mathrm{L}$, versus its fractionation level for the communications mission. For this mission, only the attributes of scalability and flexibility and their corresponding forms of responsiveness are taken into account. Therefore one could expect utility to increase with fractionation as these attributes focus on the exchange or upgrade of the payload module, which gets smaller with fractionation. The utility of the traditional spacecraft is 0.04 whereas the utility of the fractionated architectures varies from 0.38 for Architectures B and $\mathrm{C}$ to 0.5 for Architectures $\mathrm{G}$ to $\mathrm{L}$.

This figure shows that for communications missions the more fractionated the architecture is, the more utility users would derive from it. Moreover, at a given fractionation level, the number of infrastructure modules does not change the utility of the architectures.

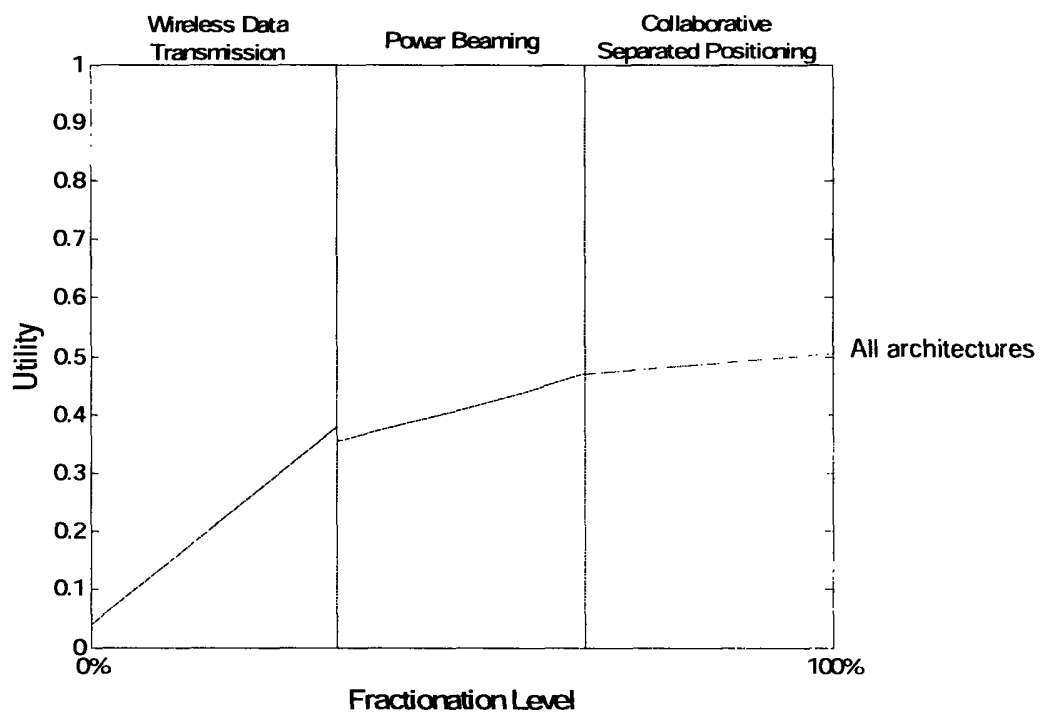

Figure 41: Utility versus fractionation level for a communications mission

Figure 42 presents the final results of the architectural analysis for the communications mission considered. It shows utility versus initial cost for each architecture. The traditional architecture $\mathrm{A}$, and the fractionated architectures $\mathrm{B}, \mathrm{C}$, and $\mathrm{G}$ to $\mathrm{L}$ are represented in this figure, as well as the architectures D, E, and F for different power fractionation level: $1 \%, 25 \%, 50 \%, 75 \%$, and $100 \%$. For this mission, cost and utility increase with fractionation, so that the general distribution of the architectures in this figure is rather simple to explain. Utility and cost increase with fractionation from the 
traditional spacecraft to the most fractionated architecture, Architecture L. The utility of the fractionated architectures increases from 0.35 to 0.5 while their cost increases by a factor 2. As noticed on Figure 41, utility is the same for all the architectures with the same fractionation level, but as can be seen in Figure 42 their initial cost varies significantly. Therefore for communications missions, the lower the number of infrastructure modules is, the cheaper and therefore the better it is.

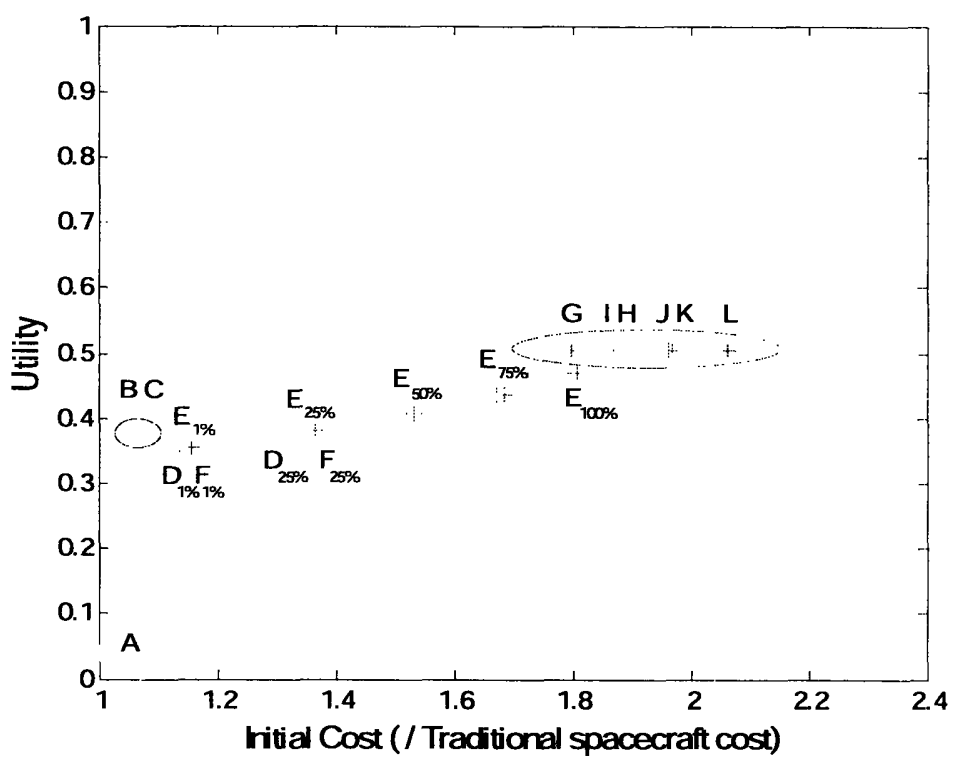

Figure 42: Utility versus initial cost for a communications mission

There are five main conclusions to this analysis of a communications mission at spacecraft level:

1) There is a significant increase in utility associated with fractionation for a very limited increase in cost for some of the fractionated architectures

2) The more fractionated the architecture is, the higher its utility

3) At a given fractionation level, the utility of all the fractionated architectures is the same, but the more infrastructure modules there are, the more expensive the architecture is

4) As fractionation increases, utility increases only from 0.35 to 0.5 , while the relative initial cost increases by $80 \%$ in the best case

5) Fractionation could be justified at spacecraft level for communications missions if the non-traditional attributes are valued enough.

\section{D.5.2 Navigation missions}

Figure 43 shows the utility of each architecture, the traditional one and the fractionated ones from $A$ to $\mathrm{L}$, versus its fractionation level for the navigation mission. For this mission, all the attributes are taken into account.

The utility of the traditional spacecraft is 0.65 whereas the utility of the fractionated architectures varies between 0.62 and 0.95 . This figure shows first that fractionated architectures can a lower utility than traditional spacecraft when investigated at spacecraft level. This figure then shows that utility initially increases with fractionation, 
but then decreases with the fractionation of the power subsystem to reach a level lower than the utility level of an equivalent traditional spacecraft, and finally remains constant when the propulsion and AOCS subsystems gets fractionated. At spacecraft level, the only architectures that have a higher utility than the equivalent traditional spacecraft are architectures $\mathrm{B}$ and $\mathrm{C}$ and architectures $\mathrm{D}, \mathrm{E}$ and $\mathrm{F}$ when the fractionation level of the power subsystem is smaller than $65 \%$. Moreover, at a given fractionation level, the number of infrastructure modules does not change the utility of the architectures, except for architectures D, E and F. One should bear in mind that these results are mostly influenced by maintainability attributes, and these conclusions are strongly connected to the choice of the power subsystem for the maintainability metric and to the assumption that if this subsystem is partly fractionated and fails only the part of the subsystem in the infrastructure would fail. As presented on Figure 29, the power module can be more expensive to exchange than the entire traditional spacecraft, and these results stress again the technological challenge associated with the power subsystem.

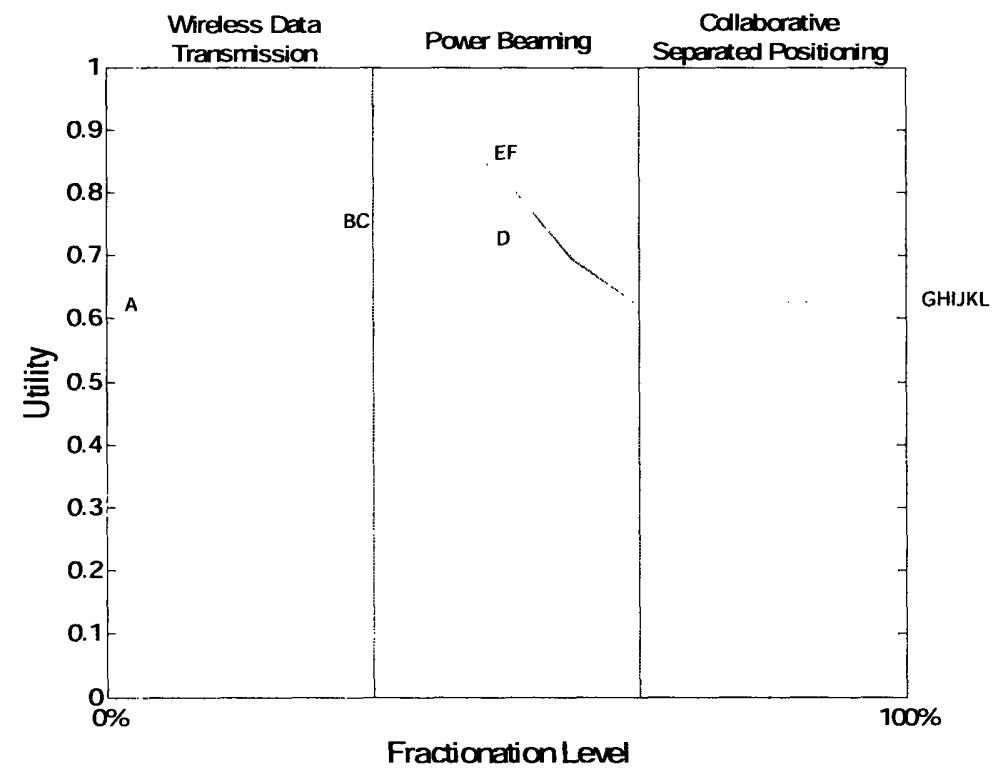

Figure 43: Utility versus fractionation level for a navigation mission

Figure 44 presents the final results of the architectural analysis for the navigation mission. It shows utility versus initial cost for each architecture. The traditional architecture $\mathrm{A}$, and the fractionated architectures $\mathrm{B}, \mathrm{C}$, and $\mathrm{G}$ to $\mathrm{L}$ are represented in this figure, as well as the architectures $\mathrm{D}, \mathrm{E}$, and $\mathrm{F}$ for different power fractionation level: $1 \%$, $25 \%, 50 \%, 75 \%$, and $100 \%$.

For this mission, cost increases with fractionation but utility does not always increase. Initially as the spacecraft gets more fractionated cost and utility increase. But once the power subsystem gets fractionated, cost keeps on increasing with fractionation but utility decreases.

At a given level of fractionation, utility is the same for all of the architectures - except for architectures D, E and F -, but cost tends to increase with the number of infrastructure modules. Thus at these levels of fractionation, customers would prefer architectures with 
the smallest number of infrastructure modules. For architectures $\mathrm{D}, \mathrm{E}$ and $\mathrm{F}$, at a given fractionation level, for instance $\mathrm{X} \%$ of fractionation of the power subsystem, cost increases with fractionation but so does utility, at least between architectures $\mathrm{D}$ and architectures $\mathrm{E}$ and $\mathrm{F}$, for percentage of fractionation that are neither too small (cf 1\%) nor too large (cf 100\%).

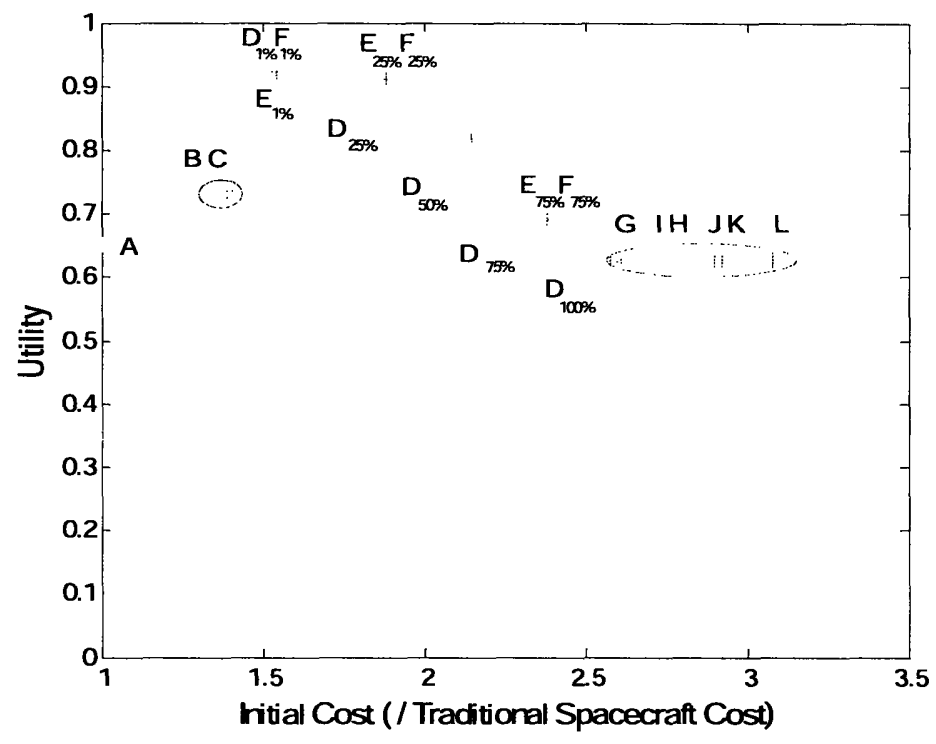

Figure 44: Utility versus initial cost for a navigation mission

There are four main conclusions to this analysis of a navigation mission at spacecraft level:

1) Fractionation increases utility only for the least fractionated architectures and at a substantial cost.

2) Once the power subsystem gets fractionated, utility decreases down to a level lower then the utility level of an equivalent traditional spacecraft while cost significantly increases with fractionation.

3) For most architectures, at a given fractionation level, the utility of all the fractionated architectures is the same, but the more infrastructure modules there are, the more expensive the architecture is

4) Fractionated spacecraft are unlikely to be a viable alternative to traditional ones at spacecraft level for navigation missions.

\section{E. Analysis at Infrastructure Level}

This chapter presents the main results of the architectural analysis at infrastructure level. Different missions and scenarios are used to illustrate the impact of fractionation on the spacecraft mass, cost, attributes, and utility. In each example, the different fractionated architectures introduced in Section B are compared with an equivalent traditional one. 


\section{E.1 Mass}

Figure 45 presents the mass penalty as a percentage of the equivalent traditional spacecraft mass for the different architectures, labeled from $\mathrm{A}$ to $\mathrm{L}$, and for two different values of the standardization parameter $s$. In the first case, $s=0 \%$. The second case, $s=$ $30 \%$, represents a situation where common infrastructure modules are overdesigned for a particular payload by $30 \%$, meaning that the infrastructure modules can support $30 \%$ more mass and power than is needed by the payload.

Using traditional mass models, the mass penalty is expected to vary in the first case between 5 and $200 \%$ from architecture B to architecture L, and in the second case between $30 \%$ and $275 \%$.

This penalty clearly increases with fractionation, except for architectures $\mathrm{G}, \mathrm{H}$, and I. As explained in Section D, this phenomenon is due to two opposite effects of fractionation on the total system mass. When a function is fractionated, the number of modules and interfaces increases, which makes the mass increase. But at the same time, as the function is no longer needed in the other modules and gets centralized, mass tends to decrease. The relative magnitudes of these two effects finally decides whether mass will increase or not with the fractionation of a function.

Figure 45 illustrates the major role played by the power function. The fractionation of the power function is obviously responsible for most of the total mass penalty of the fully fractionated architectures ( $G$ to $L)$. As mentioned in Section $D$, the main technology development emphasis has to be put on power technologies to make the fractionated spacecraft concept viable.

The standardization parameter $\mathbf{s}$ clearly has an impact on the mass penalty associated with each architecture. For instance, the architecture $\mathrm{L}$ is associated with a mass penalty of $200 \%$ for $s=0 \%$, and $275 \%$ for $s=30 \%$. One can notice that, in this example, when $s$ increases from $0 \%$ to $30 \%$, the mass penalty increases by more than $30 \%$. Moreover, the standardization parameter also has an impact on the difference of mass penalties between the various architectures. For instance, the difference in mass penalty between the architectures $\mathrm{G}$ and $\mathrm{L}$ is larger for $\mathrm{s}=30 \%$ than for $\mathrm{s}=0 \%$.

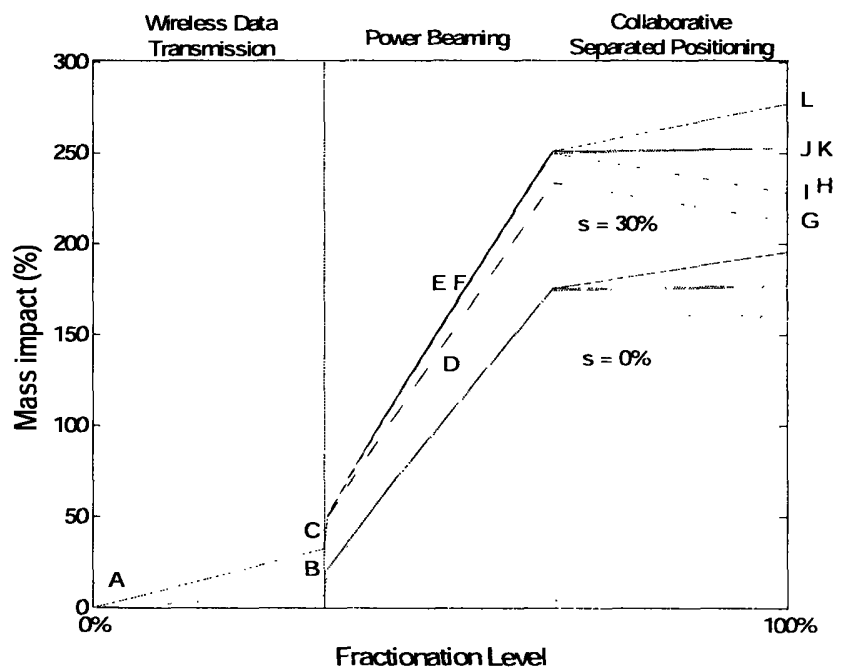

Figure 45: Mass penalty due to fractionation for different fractionated architectures 


\section{E.2 Cost}

The first example of the impact of fractionation on spacecraft initial cost is based on the communications mission introduced in Chapter $\mathrm{B}$, and the second example is based on the navigation mission also introduced in Chapter $\mathrm{B}$.

\section{E.2.1 Communications missions}

As presented above, fractionated spacecraft are expected to be heavier than equivalent traditional ones, and this mass penalty increases with the standardization parameter. On the one hand, a cost penalty is associated with this mass penalty. On the other hand, learning effects and development costs sharing tend to decrease the fractionated spacecraft costs relative to traditional spacecraft costs. These opposite effects determine the total costs of the fractionated architectures relative to the traditional ones. It is noteworthy that the learning effects and development costs sharing reduce both the development and manufacturing costs but not the launch costs, which remain directly proportional to the mass, as no learning effects are taken into account for launches. This also means that cost figures can be considered as an upper bound as one could expect at least some learning effects on launch costs if the infrastructure is developed.

Figure 46 presents the total cost impact as a percentage of an equivalent traditional spacecraft cost for all the fractionated architectures for two different values of the standardization parameter, $\mathbf{s}$. In this example, the cost impact varies between $-23 \%$ and $+17 \%$ for a standardization parameter of $0 \%$, and between $-20 \%$ and $+30 \%$ for a standardization parameter of $30 \%$.

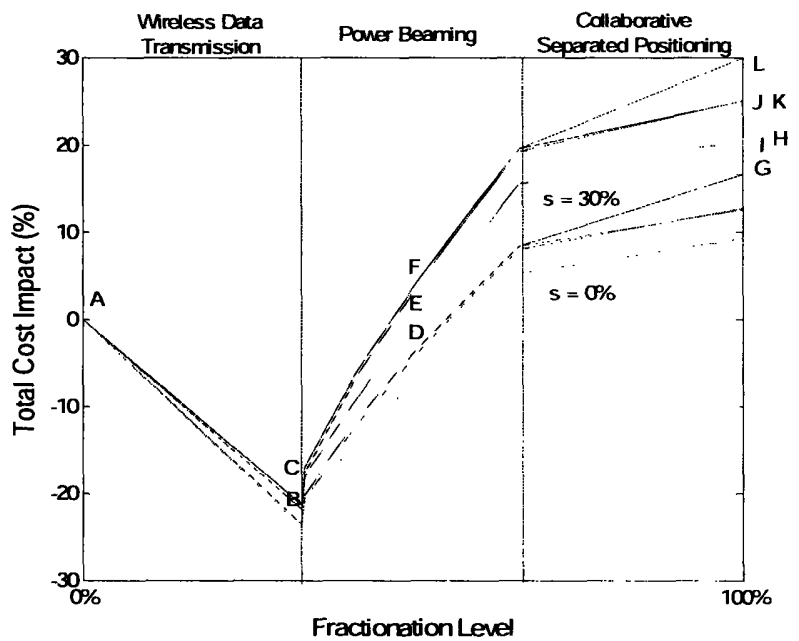

Figure 46: Impact of fractionation on the communications satellite initial cost for different architectures

The cost decrease from the traditional architecture A to the fractionated architectures B and $C$ can seem counterintuitive given the results presented on Figure 45 . The cost penalty - without any learning effects or development cost sharing - that corresponds to the mass penalty presented in Figure 45 for $s=30 \%$ is presented in Figure 47 . If learning effects and development cost sharing are included in calculating the cost penalty, these 
two effects work to decrease the costs of the fractionated architectures, while the cost of the traditional spacecraft A remains constant. This impact on the fractionated architectures' costs is represented by the arrows in Figure 47. For instance, the cost of architecture B becomes B' by including learning effects and development cost sharing, architecture D becomes D', and architecture G becomes G'. Depending on the amplitude of these effects, the fractionated architectures can be cheaper than the traditional architecture.

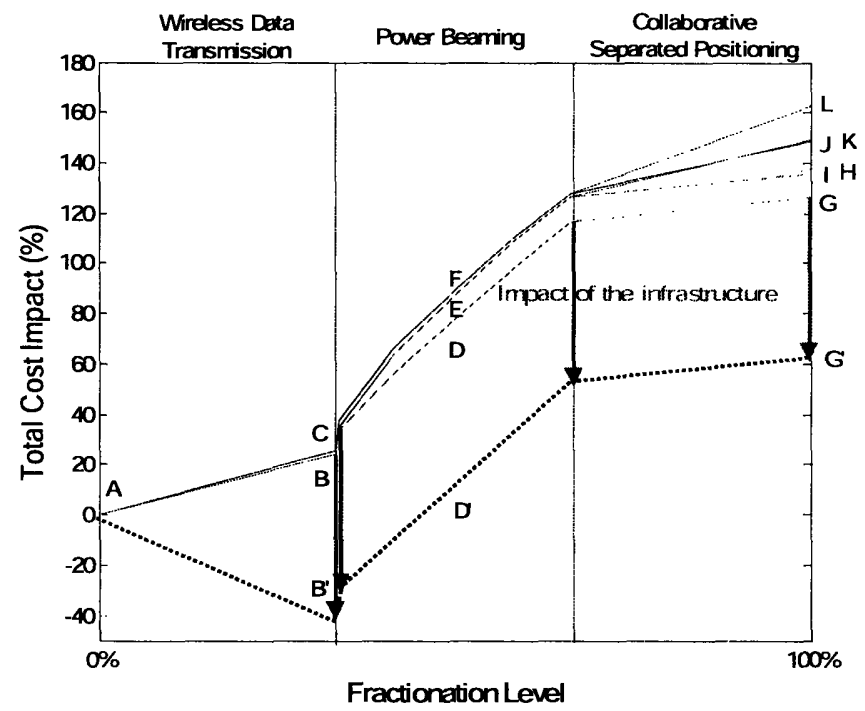

Figure 47: Impact of the infrastructure on the cost penalty for $s=30 \%$

Like for mass, the standardization parameter has an impact on both the cost penalty associated with each architecture and the difference of cost penalties between architectures. For instance, architecture $\mathrm{L}$ goes from a cost penalty of $17 \%$ for $\mathrm{s}=0 \%$ to a penalty of $30 \%$ for $\mathrm{S}=30 \%$, and this increase in cost penalty is much larger than the increase of $30 \%$ in the standardization parameter s. In addition, the difference in the cost penalty between architectures $\mathrm{G}$ and $\mathrm{L}$ increases by more than $30 \%$ when $\mathrm{s}$ goes from $0 \%$ to $30 \%$.

Architectures $\mathrm{B}$ and $\mathrm{C}$ have the lowest initial costs among the fractionated architectures and are cheaper than the traditional architectures. Then as fractionation increases, the fractionated architectures become more expensive, soon surpassing the cost of the traditional spacecraft. Again, the power is responsible for most of the cost penalty of the fully fractionated architectures.

\section{E.2.2 Navigation missions}

Figure 48 presents the total cost impact as a percentage of an equivalent traditional spacecraft cost for all the fractionated architectures for two different values of the standardization parameter, $\mathbf{s}$. This figure has similar features as the one presented above for the communications mission. For this mission, the cost impact varies between $-27 \%$ and $+15 \%$ for a standardization parameter of $0 \%$, and between $-24 \%$ and $+30 \%$ for a standardization parameter of $28 \%$. This figure does not take into account any learning effects for the traditional spacecraft. 


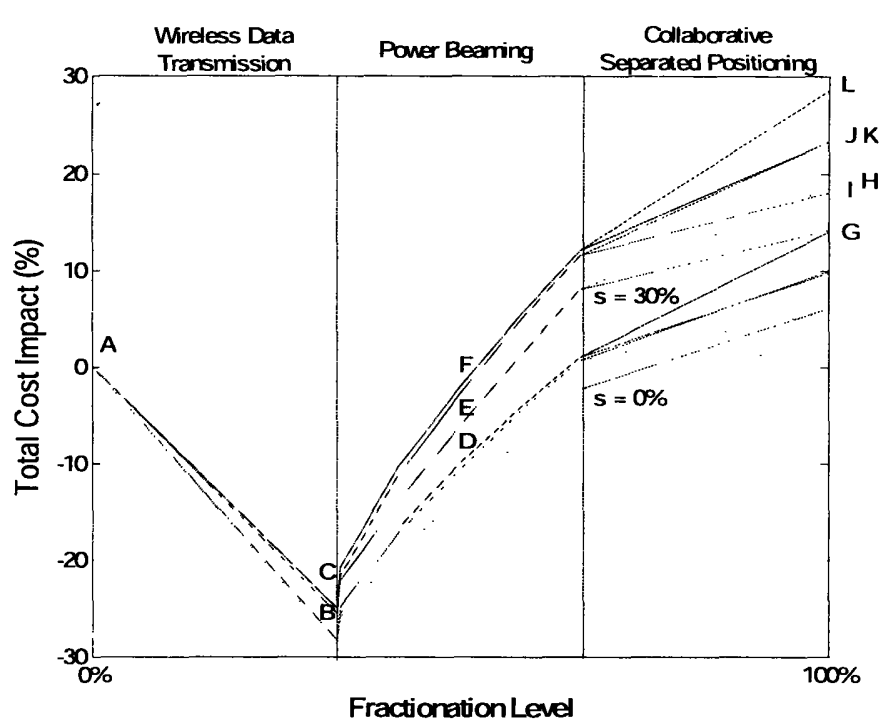

Figure 48: Impact of fractionation on the navigation satellite initial cost for different architectures

\section{E.3 Attributes}

\section{E.3.1 Maintainability}

Two examples of supporting function failure are presented to illustrate the maintainability metric at infrastructure level. A standardization parameter of $30 \%$ is assumed.

First, a failure of the communications subsystem is assumed. Figure 49 presents the maintenance cost for the different fractionated architectures as a percentage of the exchange cost for an equivalent traditional spacecraft. The maintenance cost for all fractionated architectures is significantly lower than the cost of a traditional spacecraft exchange, even for architectures D, G, and $\mathrm{H}$. In these architectures, the power subsystem is fractionated in the same module as the communications subsystem, and in fact this module becomes larger than the initial traditional spacecraft. But the learning effects applied to the infrastructure modules manufacturing more than compensates for the cost penalty linked to the increased size of the module. 


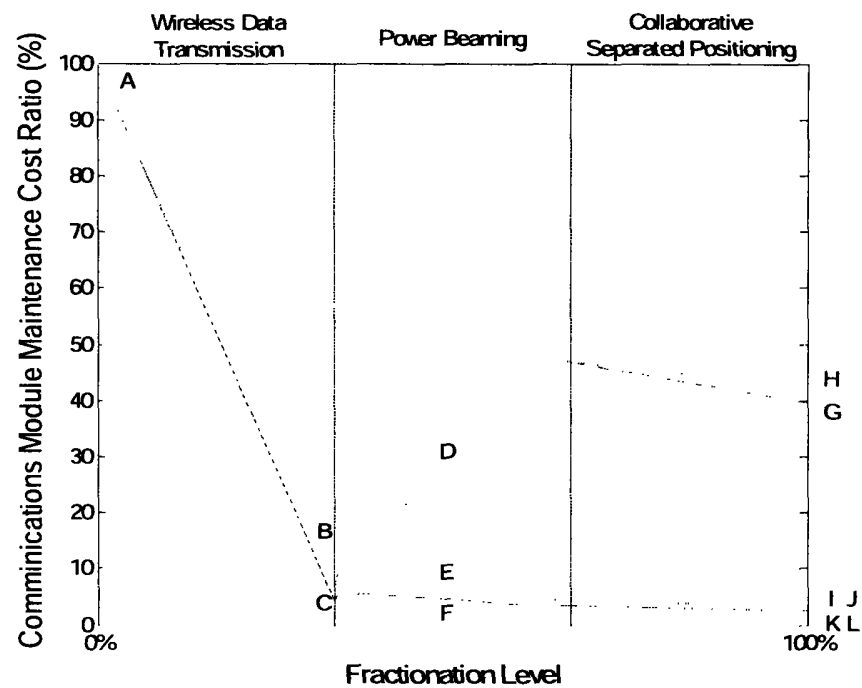

Figure 49: Cost of the communications module exchange relative to the traditional spacecraft cost (communications mission)

The second example investigates the particular case of the power module. A total failure of the power subsystem is assumed. Figure 50 presents the relative maintenance cost for all the fractionated architectures. On the left third of the figure, the module that contains the power subsystem is the payload module. Then, as the fractionation level increases, the power fractionated is either, like in architecture $\mathrm{D}$, added to the communications and data handling module, or a new power module is created, like in architectures $\mathrm{E}$ and $\mathrm{F}$. This difference explains the step when the power starts getting fractionated in the case of architecture D. For architectures D to $\mathrm{L}$, the cost to maintain the power subsystem is much lower than the cost of a traditional spacecraft. Again, the learning effects compensate for the increased mass of the module, which becomes larger than the initial traditional spacecraft.

Figure 49 and Figure 50 clearly demonstrate and quantify the improved maintainability of all the fractionated architectures relative to the traditional one. As could be expected, architectures B and C are the "most maintainable" in the case of a communications subsystem failure, whereas all the other fractionated architectures are "more maintainable" than these in the case of a power subsystem failure, which demonstrates a tradeoff to be made when choosing an architecture, depending on subsystem reliability. 


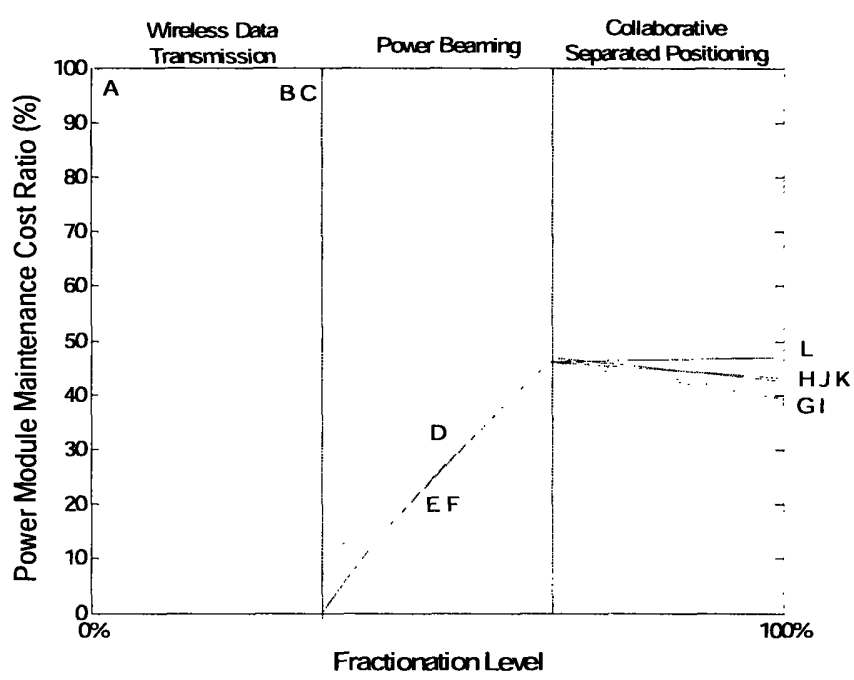

Figure 50: Cost of the power module exchange relative to the traditional spacecraft cost (communications mission)

\section{E.3.2 Scalability}

Scalability represents the ability of the system to respond to the need for an increased level of performance. To illustrate the scalability provided by spacecraft fractionation, a spacecraft with a standardization parameter $\mathrm{S}$ of $30 \%$ is assumed, which means that the existing infrastructure supporting the payload has 30\% unused capacity in the form of standardization margins that can be used to support, partly or entirely, an additional payload.

Figure 51 presents the upgrade cost relative to a traditional spacecraft exchange for different levels of performance increase needed. There is only one curve for each value of the percentage of performance increase desired, $p$, since only the payload module is being altered to achieve scalability, and not the infrastructure modules. Besides, this figure does not change with the number of units produced as only the customized payload module is taken into account.

Even when this increased performance is much larger than the margins provided by standardization, in this case $30 \%$ margins, the upgrade cost remains relatively low. One can notice that all fractionated architectures are similarly scalable relative to the traditional spacecraft. When the standardization margins are equal to or larger than the performance increase needed, the payload module is the same for all architectures, which explains why the two lower curves are flat in the right two thirds of the figure. When the standardization margins are smaller than the performance increase, the payload modules are no longer the same, and the more fractionated the architecture, the more expensive they are, though the cost increase is relatively small.

It is important at this point to summarize what factors explain the differences among the three non-traditional attributes of maintainability, flexibility and scalability. The dominance of the fractionated architectures in terms of maintainability is due both to the modularity of these architectures and the effects associated with the size of the infrastructure, i.e. learning effects and the development cost sharing. On the other hand, the dominance of the fractionated architectures in term of scalability and flexibility is 
only due to the modularity provided by fractionation. It is simply due to the fact that only what is needed is developed, manufactured, and launched.

Figure 51 demonstrates the superior scalability of the fractionated architectures. This figure also suggests that it may be worth investigating in more detail strategies of staged deployment of fractionated spacecraft in cases of uncertain increases in demand (de Weck and al., 2003) (de Weck and al., 2004).

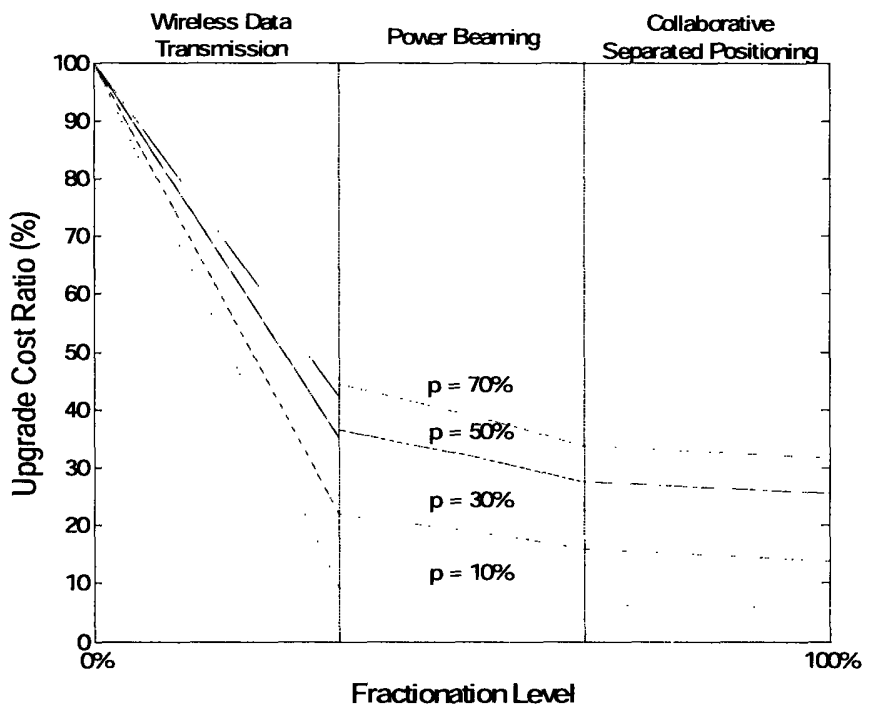

Figure 51: Cost of the additional payload module relative to the scaled-up traditional spacecraft cost for different percentages of performance increase $p$ (communications mission) Note: Each line represents all architectures

\section{E.3.3 Flexibility}

Flexibility represents the ability of the system to respond to the need for a new function. In the flexibility scenario, the payload module of the initial spacecraft is assumed to be replaced with one that has a newly-developed payload while utilizing the same infrastructure. Figure 52 presents the upgrade cost relative to an equivalent traditional spacecraft cost for different values of the standardization parameter. Like in the scalability assessment, there is only one curve for all the architectures for a given value of the standardization parameter. This figure is the same as the one presented in Section D in the analysis at spacecraft level, as the infrastructure does not change the flexibility results. The exchange cost logically decreases to less than $50 \%$ as fractionation increases. The more fractionated the architecture is, the less expensive the exchange is.

The advantage of the fractionated architectures in the flexibility example lies in the learning effects and development costs sharing. Apart from launch costs, most of the exchange cost is the development and manufacturing costs of the new payload, which underlines how the concept of fractionated spacecraft enable customers to better use their funds by paying mainly for the new value-added payload. 


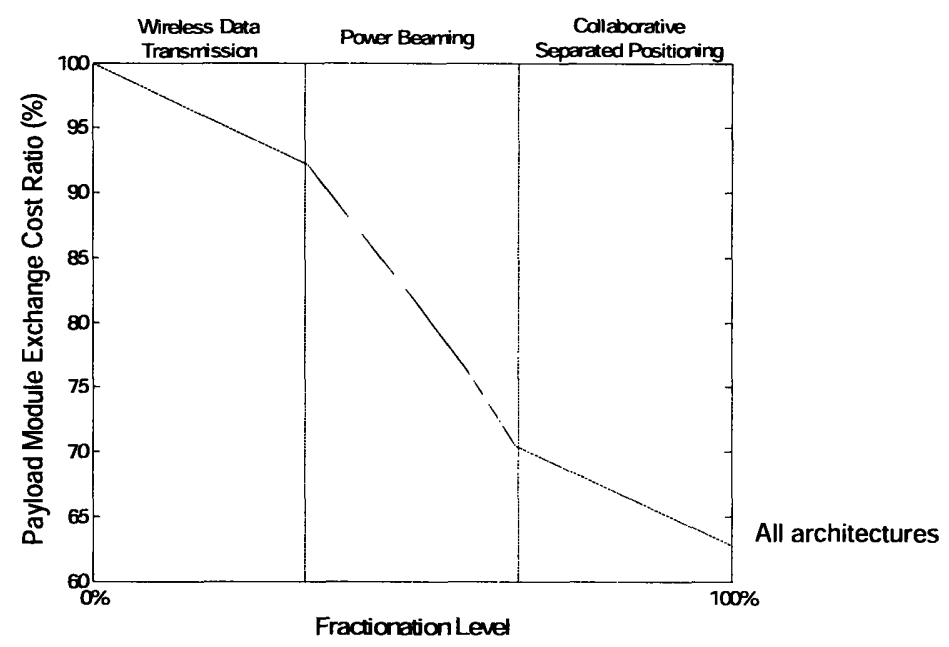

Figure 52: Cost of the new payload module relative to the traditional spacecraft cost (communications mission)

\section{E.3.4 Responsiveness associated with maintainability}

The scenario chosen to assess the architecture responsiveness associated with maintainability is the same as the one used for maintainability.

A total failure of the power subsystem is assumed. Figure 53 shows the ratio between the time necessary to manufacture, integrate, assemble and test a new module and the time necessary for a whole traditional spacecraft. In case of power subsystem failure, once the power subsystem is fractionated, the more fractionated the architecture is, the longer the time necessary to maintain it. For the least fractionated of the fractionated architectures, the time ratio between fractionated and traditional architectures is lower than $10 \%$, whereas for the most fractionated spacecraft this ratio is around $50 \%$. Again, the learning effects compensate for the increased mass of the module, which becomes larger than the initial traditional spacecraft. It is noteworthy that this result, and the following ones, is based on the hypothesis that the fractionated spacecraft will have comparable development, manufacturing, integration, and test cycles as a traditional spacecraft, which is unfavorable to the fractionation concept even when looking only at the payload module. 


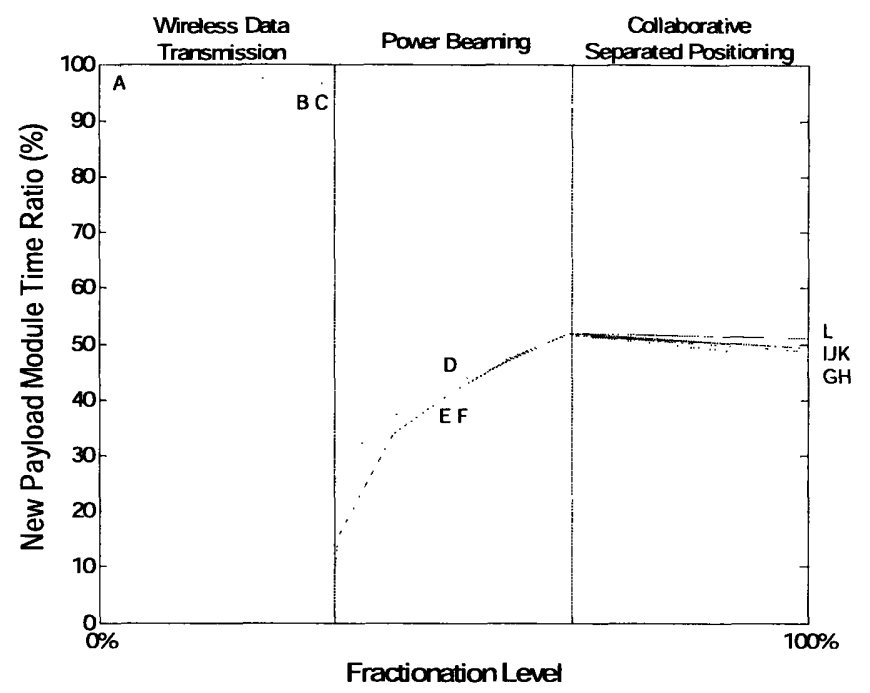

Figure 53: Time necessary to exchange the power module relative to the traditional spacecraft (communications mission)

\section{E.3.5 Responsiveness associated with scalability}

The scenario chosen to assess the architecture responsiveness associated with scalability is the same as the one used for scalability.

Figure 54 shows the ratio between the time necessary to develop, manufacture, integrate, assemble and test an additional module and the time necessary for a whole scaled-up traditional spacecraft. This ratio gets as low as $40 \%$ for a $10 \%$ increase in the performance level, and is still lower than $63 \%$ for an increase of $70 \%$. The relative gain in time is clearly less than proportional to the increase, $p$.

The time necessary to get the increased performance level generally decreases with fractionation, in a similar way for different value of performance increase, p. There is again a step when the power is fractionated, as already seen above, so that the less fractionated Architectures D, E, and F are less "responsive" than Architectures B and C. 


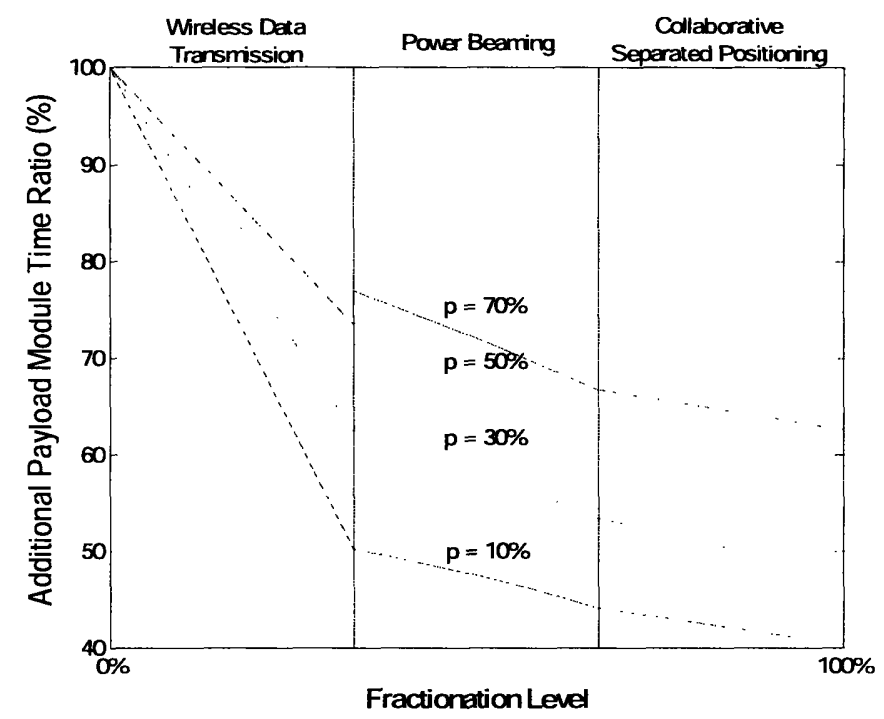

Figure 54: Time necessary to get the additional payload module relative to the scaled-up traditional spacecraft for different percentages of performance increase $p$ (communications mission) Note: Each line represents all architectures

\section{E.3.6 Responsiveness associated with flexibility}

The scenario chosen to assess the architecture responsiveness associated with flexibility is the same as the one used for flexibility.

Figure 55 shows the ratio between the time necessary to develop, manufacture, integrate, assemble and test a new payload module and the time necessary for a whole traditional spacecraft. This figure is the same as the one presented in Section D in the analysis at spacecraft level, as the infrastructure does not change the flexibility results. This ratio gets as low as $70 \%$ for the most fractionated architectures.

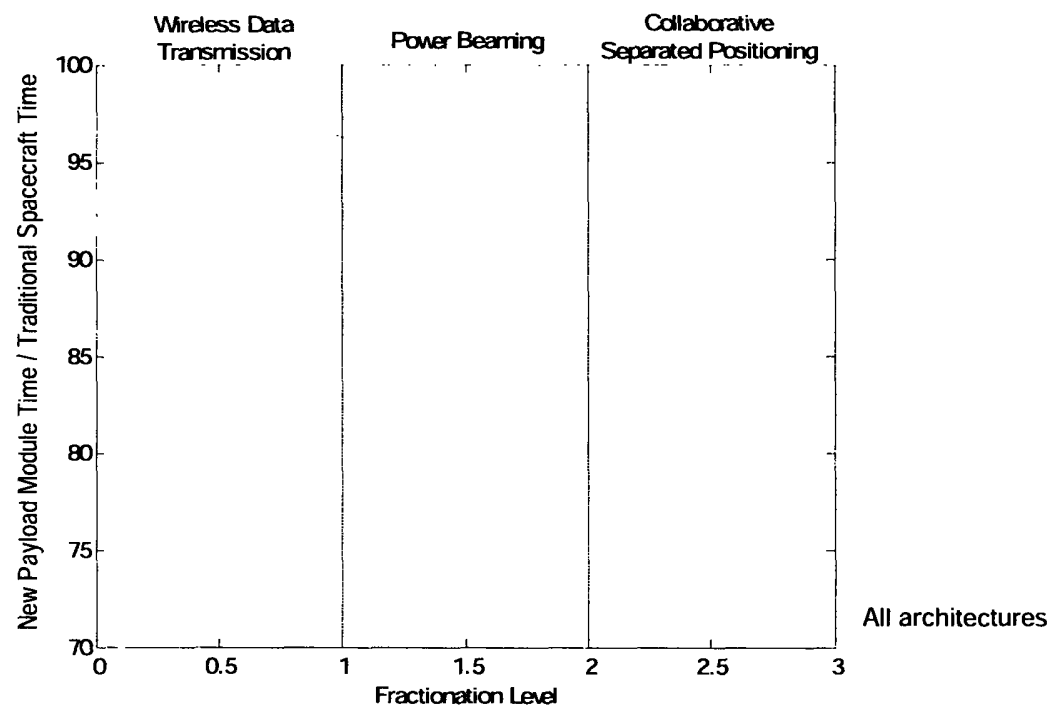

Figure 55: Time necessary to get a new payload module relative to the traditional spacecraft (communications mission) 


\section{E.4 Net Present Value}

If the function delivered by space systems has a market value, the costs to maintain the satellite or to upgrade or exchange its payload module can be associated with revenues in order to compute the net present value of the spacecraft for different scenarios. Thus a net present value distribution can be computed for each architecture for the attributes of maintainability, scalability, and flexibility. The example used in this section is the communications satellite defined in Chapter B, considered as commercial.

\section{E.4.1 Maintainability}

If, for a given subsystem, the maintainability cost results are associated with an expected revenue flow and a set of failure scenarios, the resulting distribution of net present values for the different architectures can be drawn. The failure of the power subsystem, which is one of the most frequent failures, is used as an example. For a $90 \%$ reliability of the power subsystem over the spacecraft lifetime, the net present value (NPV) of the spacecraft revenue has a $90 \%$ chance to reach its maximum value. This maximum value depends on the expected value of the revenue flow and the initial costs. Therefore, as can be concluded from Figure 47, Architectures $B$ and $C$ will have the largest maximum net present value, followed by the traditional spacecraft, and then the other fractionated architectures. But one should also look at what happens in the $10 \%$ chance that corresponds to the risk of failure. In the case of a failure of the power subsystem, the traditional spacecraft is unlikely to be exchanged. As a result, the revenues generated by the spacecraft will drop to zero. But as fractionated spacecraft can be maintained at much lower costs, they are more likely to be maintained, which enable customers to go on deriving revenues from the spacecraft. Therefore the NPV decreases in the $10 \%$ chance of failure much less for the fractionated architectures than for the traditional one as illustrated on the notional curves of Figure 56. On this figure, the plain line represents a notional cumulative probability distribution of the NPV for the traditional architecture; the dashed line represents one for architecture B; and the dotted line represents one for architecture G. This difference shows how fractionated spacecraft enable customers to capture revenues that otherwise might be lost, and this can be measured using the concept of value at risk. The value at risk (VAR), introduced in (Hassan and al., 2005), is the minimum value one can gain or maximum value one can loose at a given level of confidence. In the case of fractionated spacecraft, the minimum value at risk for a given level of confidence tends to be larger for all the fractionated architectures than for the traditional spacecraft. Figure 56 shows that even though the traditional architecture may have a larger NPV than some of the fractionated architectures, its VAR, here for a $95 \%$ level of confidence, tends to be lower than the all the VAR of the fractionated architectures.

In calculating the NPV cumulative probability distributions and VAR for each of the fractionated architectures, the first conclusion is that architectures $\mathrm{B}$ and $\mathrm{C}$ completely dominate the traditional architecture as they both have a larger NPV and a larger VAR for a high level of confidence. The architectures D to L, which are initially a little more expensive, have a lower maximum NPV than the traditional spacecraft but a much larger VAR at a high level of confidence. These benefits provided by fractionation are directly proportional to the reliability of the subsystem. Thus, an interesting trade-off can be made 
between reliability and maintainability for the fractionated spacecraft. A lower reliability would enable designers to reduce costs, and increase the maintainability of fractionated spacecraft relative to traditional ones, and this would increase their NPV and VAR differences with traditional spacecraft.

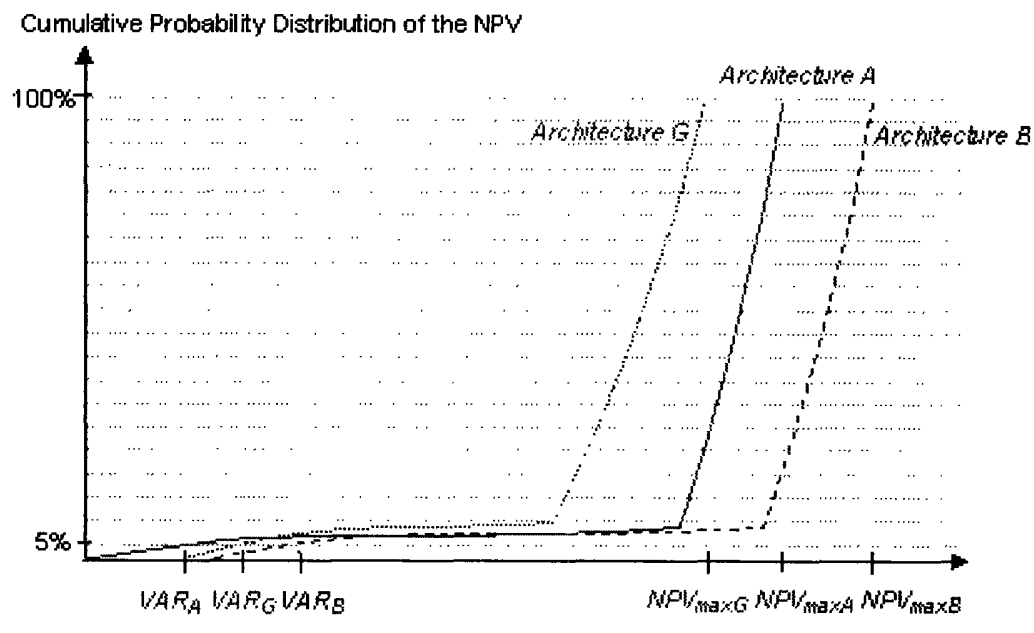

Net Present Value (NPV)

Figure 56: Value at risk for a 95\% confidence level and maximum net present values for different architectures in evaluating maintainability

\section{E.4.2 Scalability}

If the scalability cost results are associated with a revenue flow and demand scenario, the distribution of net present values can be drawn for the different architectures. When compared, these distributions look like the notional curves presented on Figure 57. The main difference between the traditional architecture, represented by the plain line, and the fractionated architectures, represented by dashed lines, is an increase in the maximum NPV. When scalability is evaluated, fractionation mainly "stretches" the distribution towards larger values. This illustrates that fractionated architectures can be viewed as an option to capture revenue from the upside uncertainty in demand.

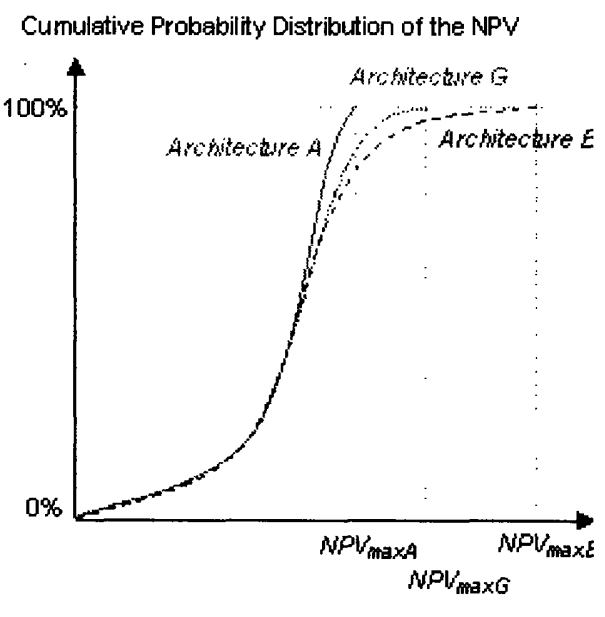

Figure 57: Notional net present value cumulative probability distribution for different architectures in evaluating scalability and flexibility 


\section{E.4.3 Flexibility}

If these flexibility cost results are associated with a revenue flow and a set of demand scenarios, the corresponding distribution of net present values can be drawn for the different architectures. The same phenomenon as the one described in the scalability assessment and illustrated on Figure 57 is observed. Again, fractionation enables customers to capture new revenues, which increases the spacecraft maximum net present value.

\section{E.5 Utility}

If no market can be associated with the value delivered to users by space systems, utility is used. Different utility figures based on the communications and navigation missions introduced in Chapter B are presented below. These utility figures for each attribute are based on the single-utility functions as defined by the communications expert.

All the figures presented above in this section were relative figures, i.e. values for the fractionated architectures relative to the value for the equivalent traditional architecture, whereas the ones that follow are absolute figures.

\section{E.5.1 Maintainability}

Figure 58 shows the utility associated with maintainability of the different architectures. This figure corresponds to the failure of the power subsystem. As mentioned above, these results are based on the assumption that if the power subsystem is partly fractionated, the part of the system that is assumed to fail is the one located in the module containing the power subsystem.

Fractionation significantly increases the utility associated with the maintainability of the spacecraft: while the utility of the traditional architecture is 0.46 , the utility of the fractionated architectures varies between 0.58 and 1 . One could expect such results, even with the power subsystem, as the development of the infrastructure significantly impacts the cost of the infrastructure modules. This impact of the infrastructure can be appreciated when comparing this figure with Figure 35 which corresponds to the analysis at spacecraft level.

As presented on Figure 29 for the communications mission, the cost of maintaining the module containing the power subsystem initially decreases with fractionation, but as the fractionation of the power subsystem increases this cost increases. These trends are similar for navigation missions. Thus the utility associated with the maintainability of the spacecraft for the power subsystem increases from the traditional architecture to architecture $\mathrm{B}$ and $\mathrm{C}$. It also increases when the power starts getting fractionated but then decreases with the fractionation of the power subsystem. Once the power subsystem is fractionated, utility either increases or decreases with the fractionation of the AOCS and propulsion subsystems. At a given fractionation level, utility varies with the different architectures. The more infrastructure modules there are, the lower the utility of the architecture is. 


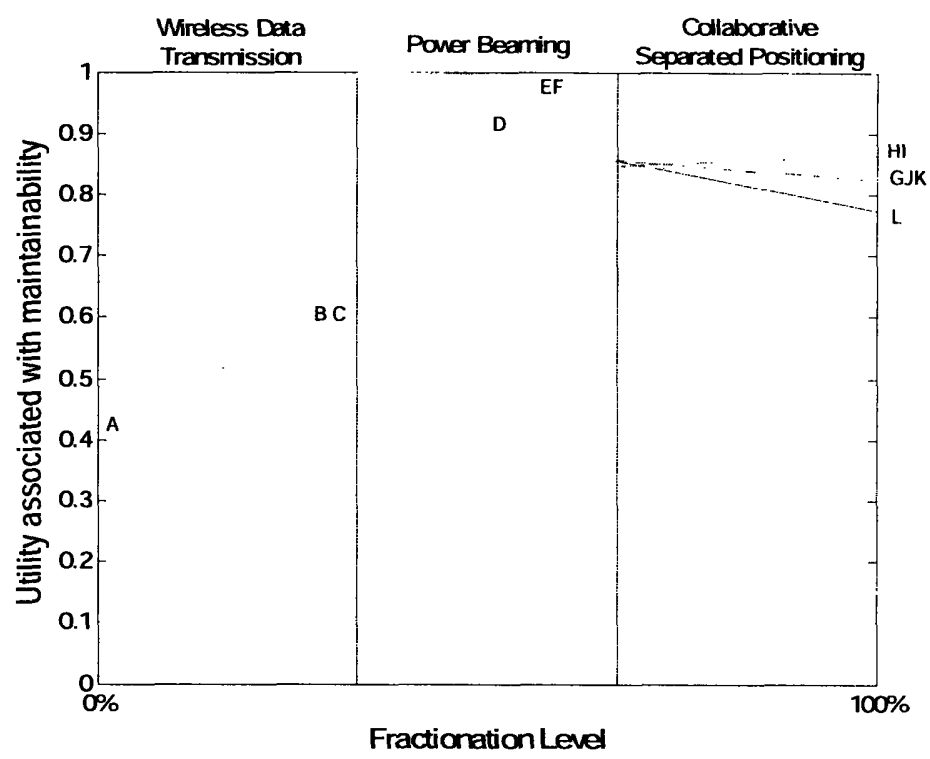

Figure 58: Utility associated with maintainability (navigation mission)

\section{E.5.2 Scalability}

Figure 59 shows the utility associated with scalability of the different architectures for a percentage of performance increase $\mathrm{p}$ of $50 \%$ and a standardization parameter $\mathrm{s}$ of $30 \%$. Again, there is only one line for all the architectures as only the payload module, which is similar for the architectures with the same fractionation level, influences the utility value. The utility of the traditional architecture (which is scaled-up by $50 \%$ ) is 0 and the utility of the fractionated architectures varies between 0.61 and 0.72 . As expected the more fractionated the architecture is, the higher the utility.

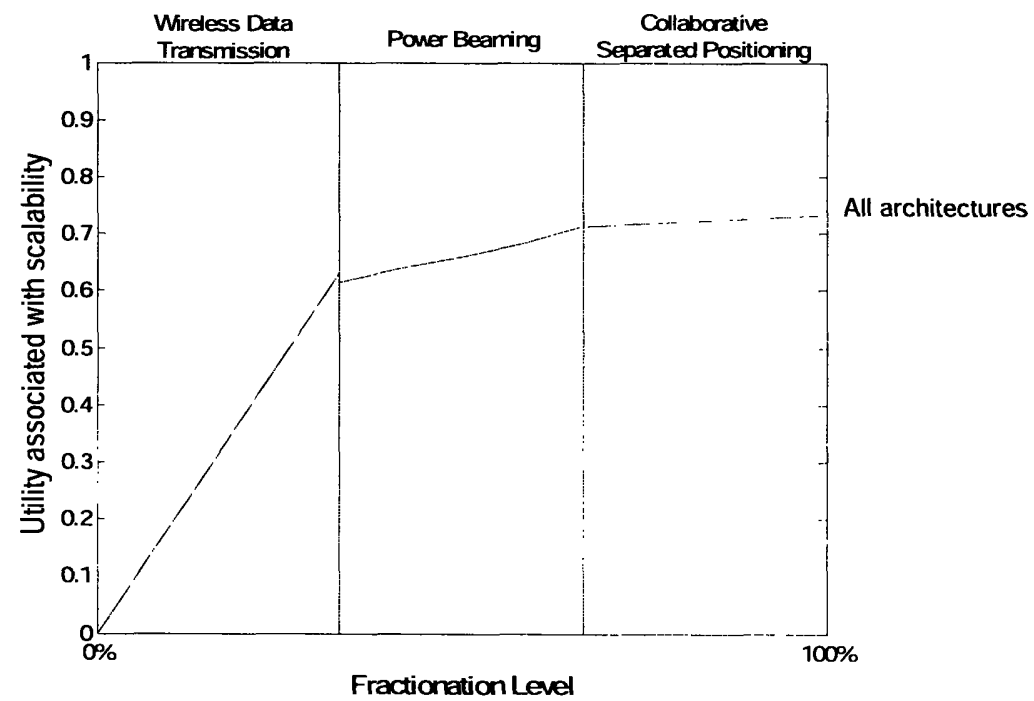

Figure 59: Utility associated with scalability for $\mathrm{p}=50 \%$ and $s=30 \%$ (communications mission) 


\section{E.5.3 Flexibility}

Figure 60 shows the utility associated with flexibility of the different architectures. This figure is similar to the one presented in the analysis at spacecraft level, as only the payload module is considered independently from the architecture of the infrastructure. The utility of the traditional architecture is 0.13 and the utility of the fractionated architectures significantly varies between 0.2 and 0.45 . As expected the more fractionated the architecture is, the higher the utility.

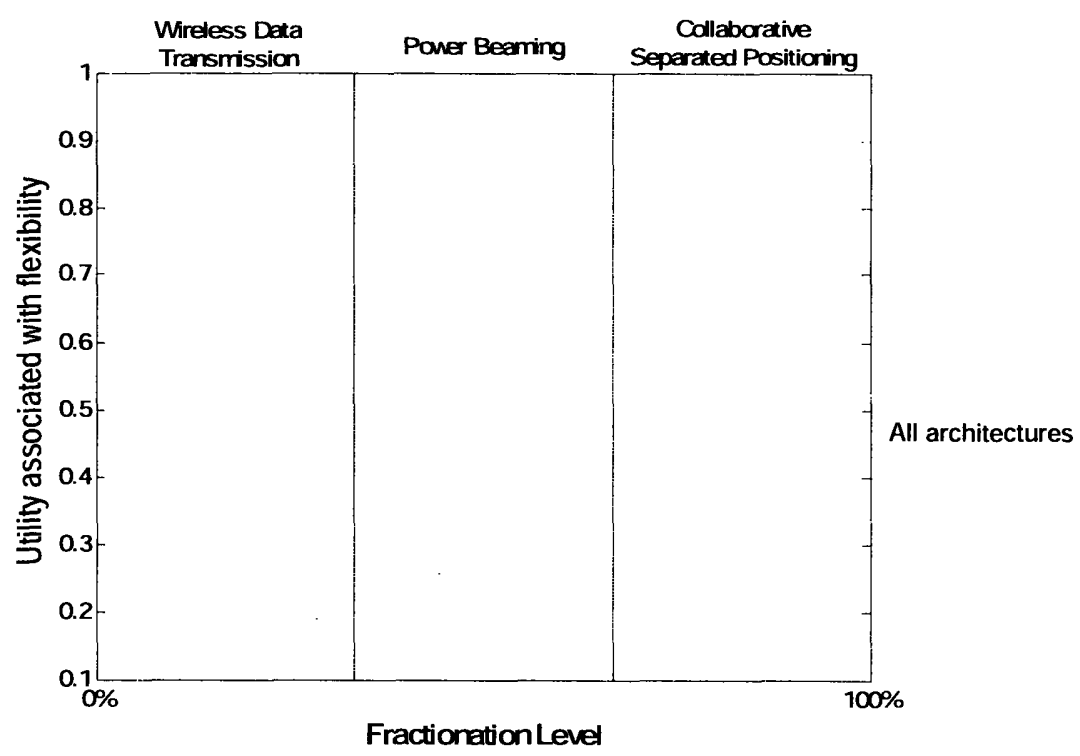

Figure 60: Utility associated with flexibility (communications mission)

\section{E.5.4 Responsiveness associated with maintainability}

Figure 61 shows the utility associated with responsiveness of the different architectures. This figure corresponds to the failure of the power subsystem. The utility of the traditional architecture and of most fractionated architectures is 0 and so is the one of the architectures B and C. But when the power subsystem gets fractionated, utility increases up to 1 and then slightly decreases with fractionation down to a level higher than 0.98 for all of the architectures. The comparison of this figure with Figure 38 shows the huge impact of the learning effects associated with the development of the infrastructure. 


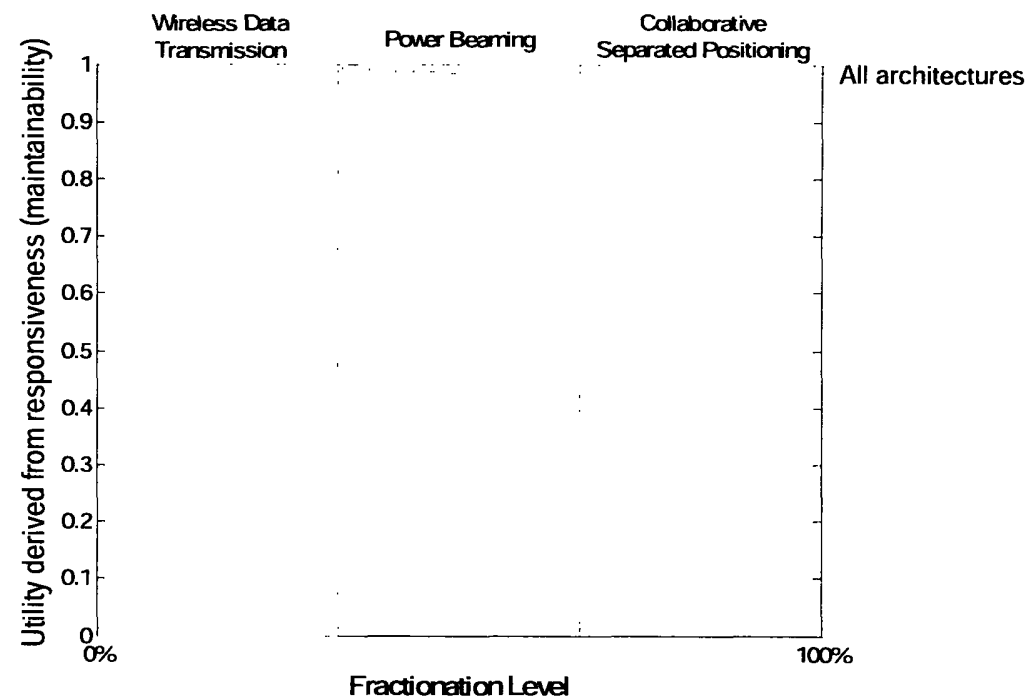

Figure 61: Utility associated with responsiveness (navigation mission)

\section{E.5.5 Responsiveness associated with scalability}

Figure 62 shows the utility associated with responsiveness of the different architectures for a percentage of performance increase $\mathrm{p}$ of $50 \%$ and a standardization parameter $\mathrm{s}$ of $30 \%$. The utility of the traditional architecture is 0 and the utility of the fractionated architectures varies between 0.27 and 0.41 . As expected the more fractionated the architecture is, the higher the utility.

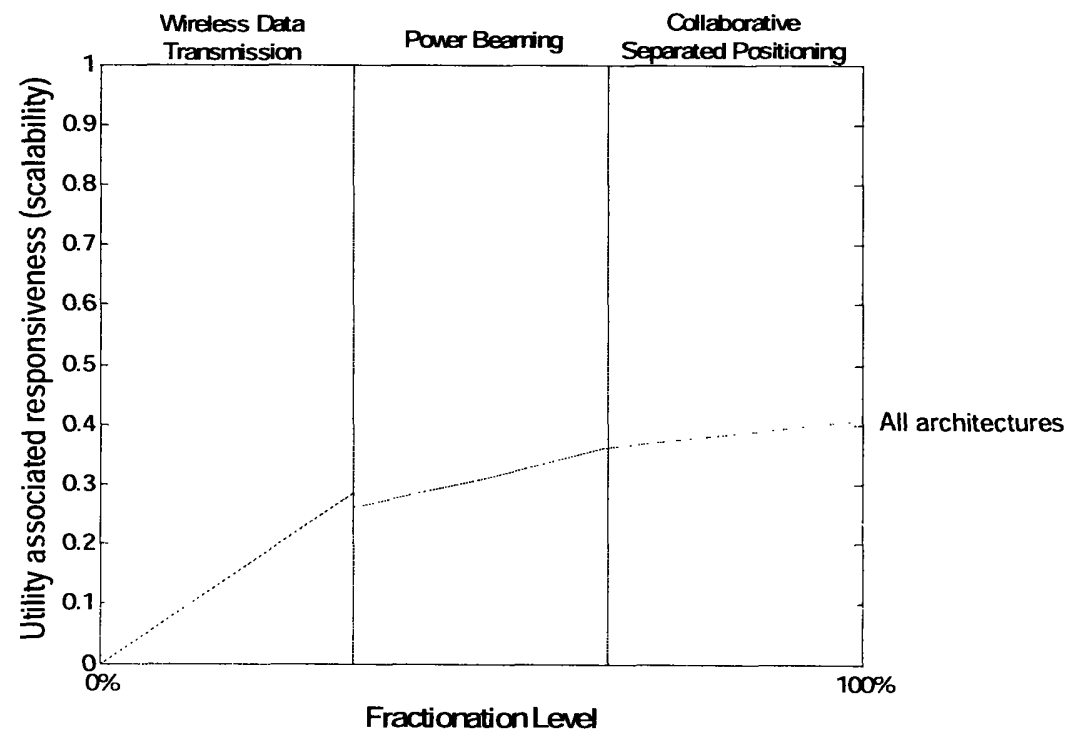

Figure 62: Utility associated with responsiveness for $\mathrm{p}=50 \%$ and $\mathrm{s}=30 \%$ (communications mission)

\section{E.5.6 Responsiveness associated with flexibility}

Figure 63 shows the utility associated with responsiveness of the different architectures. This figure is similar to the one presented in the analysis at spacecraft level, as only the 
payload module is considered independently from the architecture of the infrastructure. The utility of the traditional architecture is 0.13 and the utility of the fractionated architectures significantly varies between 0.15 and 0.40 . As expected the more fractionated the architecture is, the higher the utility.

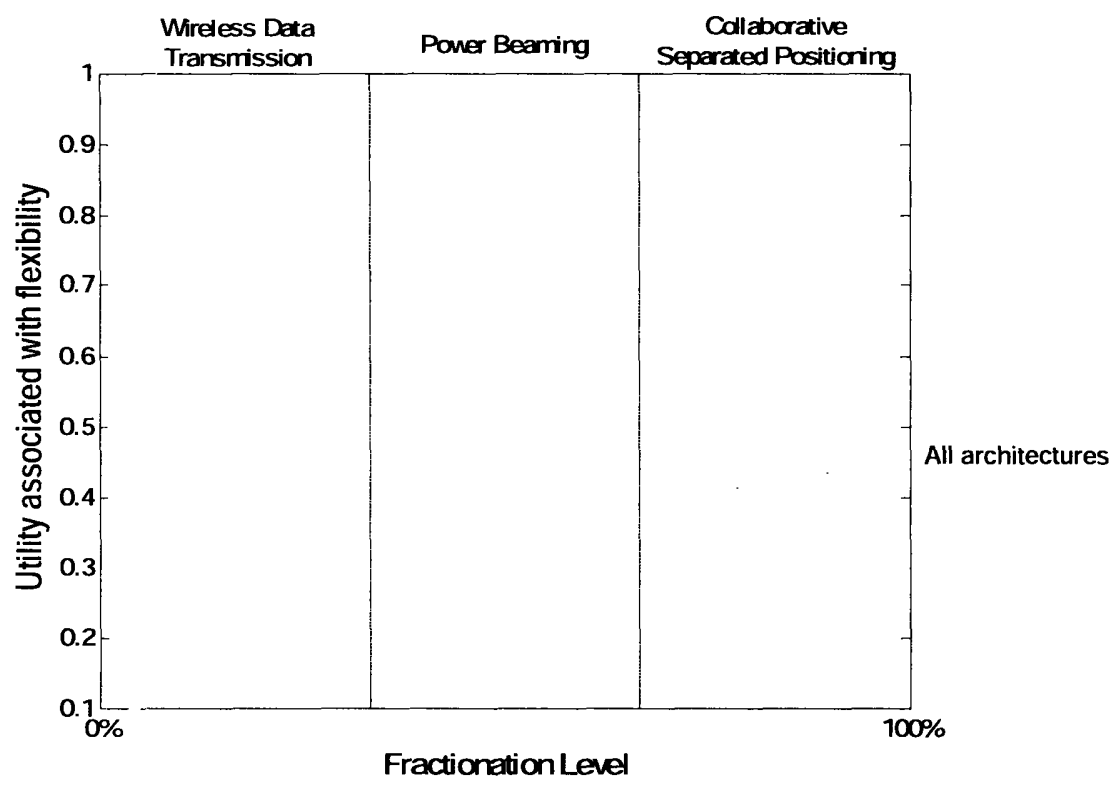

Figure 63: Utility associated with responsiveness (communications mission)

\section{E.6 Multi-attribute utility}

The utility figures presented in this section are based on the communications and navigation missions introduced in Chapter B. These utility figures include all the attributes and are based on multi-attribute utility functions as defined by the communications and navigation experts.

\section{E.6.1 Communications missions}

Figure 64 shows the utility of each architecture versus its fractionation level for different values of the standardization parameter $s$ for the communications mission. In this example the performance parameter $\mathrm{p}$ is $50 \%$. For communications missions, only the attributes of scalability and flexibility and their corresponding forms of responsiveness are taken into account. Therefore one could expect utility to increase with fractionation as these attributes focus on the exchange or upgrade of the payload module, which gets smaller with fractionation.

The standardization parameter $S$ affects the overall utility, as it has an impact the utilities associated with scalability and the corresponding form of responsiveness. For all the values of $s$ equal to or larger than $p$, the scalability and responsiveness remain the same, and so does the multi-attribute utility. For $\mathrm{s}=10 \%$, the traditional spacecraft utility is 0.05 whereas the utility of the fractionated architectures varies from 0.38 for Architectures $\mathrm{B}$ and $\mathrm{C}$ to 0.5 for Architectures $\mathrm{G}$ to $\mathrm{L}$. For $\mathrm{s}=50 \%$ or more, the utility of the fractionated architectures varies from 0.4 for Architectures B and C to 0.55 for Architectures $G$ to $L$ 
This figure shows that for communications missions the more fractionated the architecture is, the more utility users would derive from it. Moreover, at a given fractionation level, the number of infrastructure modules does not change the utility of the architectures.

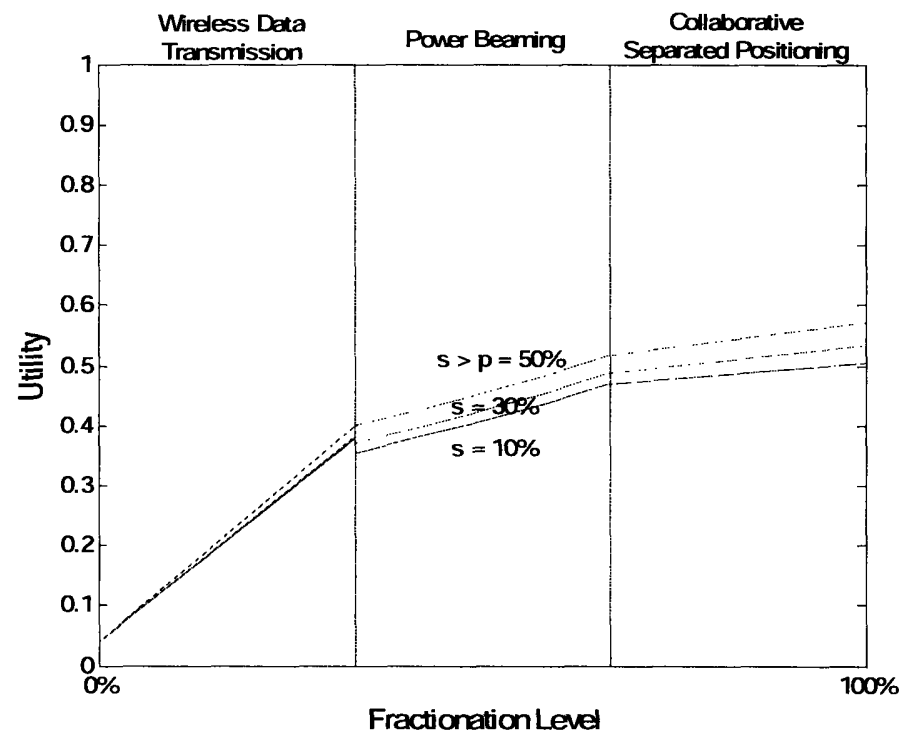

Figure 64: Utility versus fractionation level for different standardization parameter s Note: Each line represents all architectures

Figure 65, Figure 66 and Figure 67 present the final results of the architectural analysis for the communications mission. These three figures correspond to different values of the standardization parameter $s$ and different number of units produced. The traditional architecture $A$ and the fractionated architectures $B, C$, and $G$ to $L$ are represented in this figure, as well as the architectures $\mathrm{D}, \mathrm{E}$, and $\mathrm{F}$ for different power fractionation level: $1 \%$, $25 \%, 50 \%, 75 \%$, and $100 \%$.

First, Figure 65 shows the architectures utility versus their initial cost for $s=30 \%$ and 25 units produced. Utility increase with fractionation, and so does the initial cost of the fractionated architectures, but the cost of some of the fractionated architectures is lower than the traditional spacecraft cost. The utility of the fractionated architectures increases from 0.35 to 0.54 from Architecture B to L, while their cost increases from 0.83 to 1.45 times the traditional spacecraft cost. Utility is the same for all the architectures with the same fractionation level, their initial cost vary significantly. Therefore for communications missions, the lower the number of infrastructure modules is, the cheaper and therefore the better it is.

Then, Figure 66 shows the architectures utility versus their initial cost for $s=30 \%$ and 100 units produced. This figure shows clearly the benefits of fractionation. For this mission, the traditional architecture A has a utility of 0.05 . The fractionated architectures $B$ has a utility of 0.38 and is $23 \%$ cheaper than the traditional architecture. The fractionated architecture D with $100 \%$ fractionation of the power has a utility of 0.49 and is less than $12 \%$ more expensive than the traditional architecture. Finally, the fractionated architecture $G$ has a utility of 0.53 and is less than $14 \%$ more expensive than the 
traditional architecture. As can be seen on these two figures, as the number of units produced increases, the utility of the architectures does not vary but their cost significantly decreases. The cost of the least fractionated and cheapest architectures decreases less than the cost of the most fractionated and expensive ones.

Finally, Figure 67 shows the architectures utility versus their initial cost for $s=0 \%$ and 100 units produced. If Figure 66 is compared with Figure 67, as s decreases, spacecraft gets initially cheaper but becomes "less scalable", so that both initial cost and the utility decrease. Both variations affect more the most fractionated architectures than the least fractionated ones.

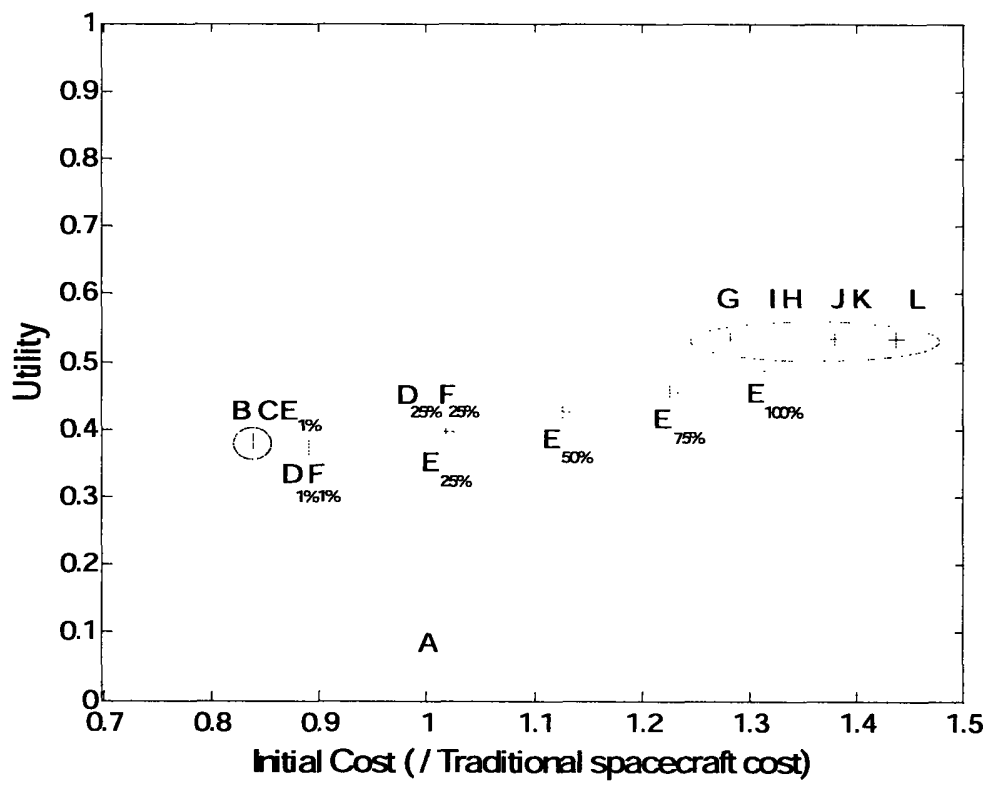

Figure 65: Utility versus initial cost for $\mathrm{s}=30 \%$ and 25 units produced

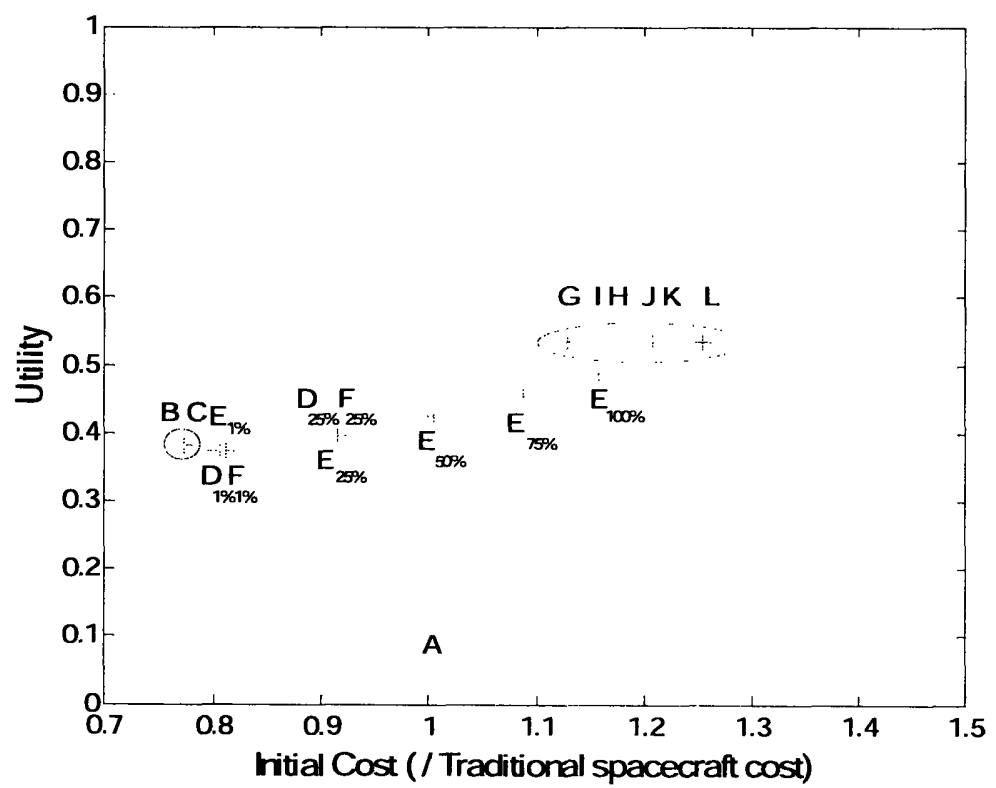

Figure 66: Utility versus initial cost for $s=30 \%$ and 100 units produced 


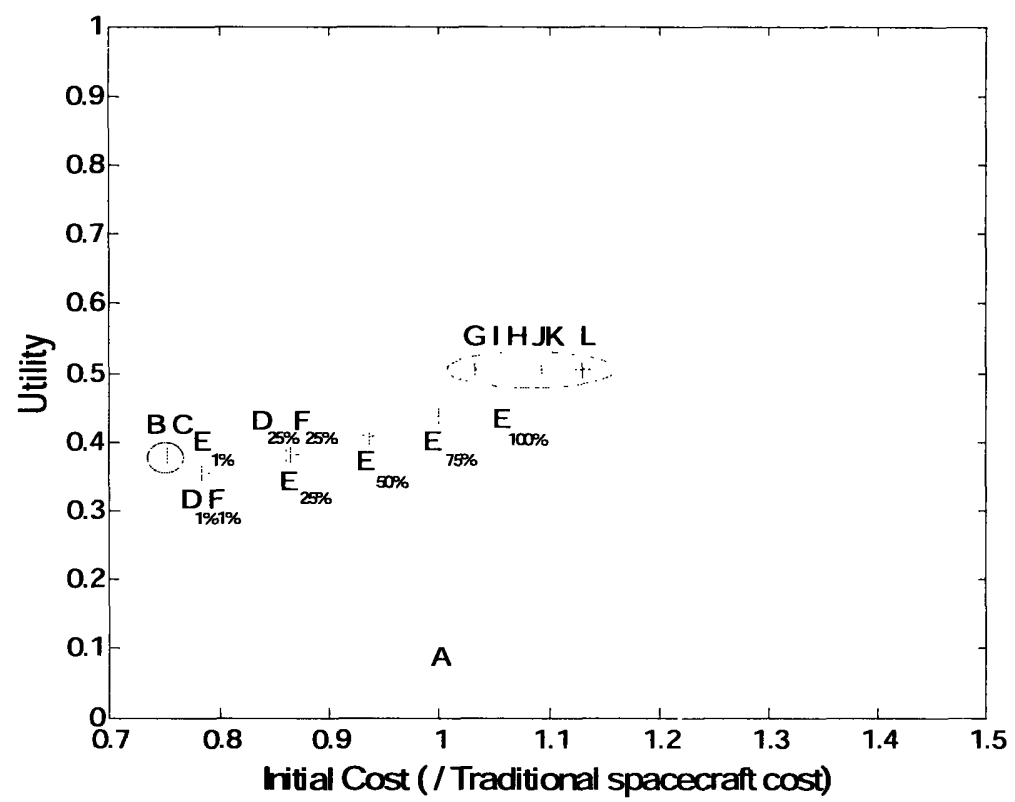

Figure 67: Utility versus initial cost for $s=0 \%$ and 100 units produced

There are seven main conclusions to this analysis of a communications mission at infrastructure level:

1) There is a significant increase in utility associated with fractionation (if fractionated and traditional architectures are compared)

2) The more fractionated the architecture is, the higher its utility it is

3) The more fractionated the architecture is, the more expensive it is

4) Depending on the number of units produced and the standardization parameter, the least fractionated architectures can be cheaper than the traditional one

5) At a given fractionation level, the utility of all the fractionated architectures is the same, but the more infrastructure modules there are, the more expensive the architecture is; Architectures B, D or G are the "best" fractionated architectures for this mission

6) As the number of units produced increases, costs decrease while utilities remain constant

7) Both cost and utility increase with the standardization parameter

\section{E.6.2 Navigation missions}

Figure 68 shows the utility of each architecture versus its fractionation level for the navigation mission and for a standardization parameter $s$ of $30 \%$. For navigation missions, all the attributes are taken into account.

The standardization parameter $s$ affects only marginally the overall utility, as this parameter is very important for scalability, but in the navigation mission case "more of the current function" is associated with a whole new satellite and not only a additional payload module that could benefit from an over-sizing of the infrastructure modules. Therefore, the standardization parameter in the navigation mission has mostly an impact 
on the cost of the infrastructure module that is exchanged in case of failure. The overall spacecraft utility is for all the fractionated architectures higher than for the traditional one. This result can be contrasted with the results of the analysis at spacecraft level. In addition, this overall utility is clearly strongly influenced by the maintainability attributes. Utility significantly increases initially with fractionation, but once the power subsystem gets fractionated, it tends to decrease a little. While the traditional spacecraft has an overall utility of 0.65 , architectures $B$ and $C$ have utilities of 0.72 , architectures $D$, $E$ and $F$ have utilities that vary between 0.9 and 0.95 , and architectures $G$ to $L$ have utilities that vary between 0.88 and 0.91 . Finally, at a given fractionation level, the number of infrastructure modules only slightly changes the utility of the architectures.

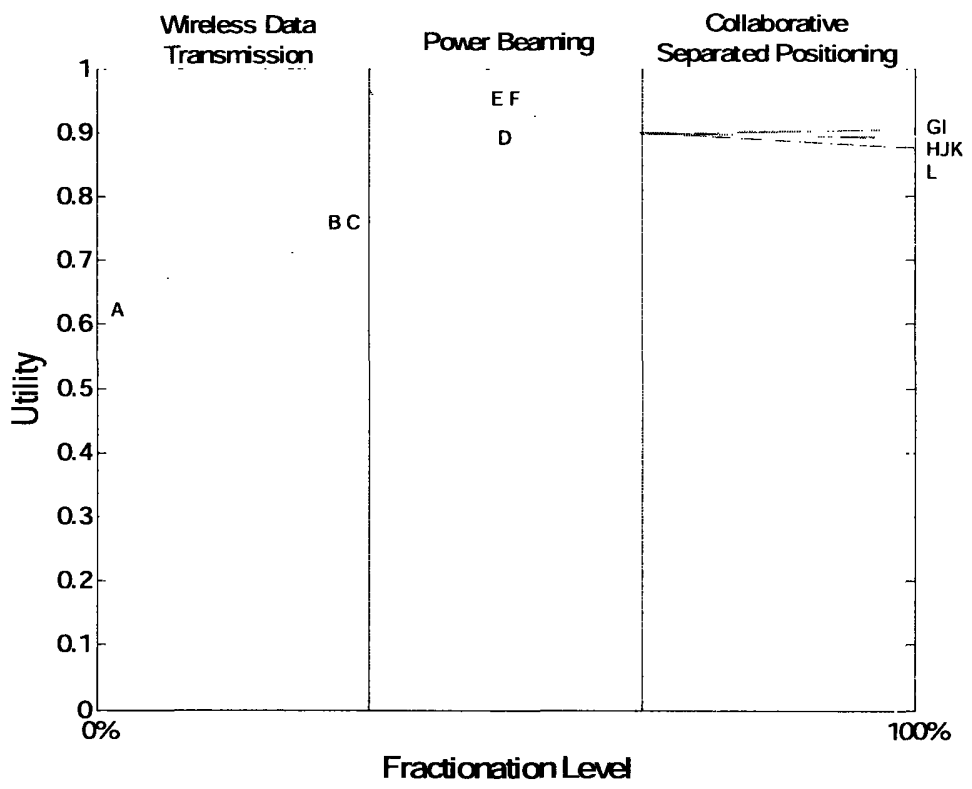

Figure 68: Utility versus fractionation level for a navigation mission for $s=30 \%$ and 100 units

Figure 70, Figure 72 and Figure 74 present the final results of the architectural analysis for the navigation mission. These three figures correspond to different values of the standardization parameter $\mathrm{s}$ and different number of units produced. The traditional architecture $A$ and the fractionated architectures $B, C$, and $G$ to $L$ are represented in this figure, as well as the architectures $\mathrm{D}, \mathrm{E}$, and $\mathrm{F}$ for different power fractionation level: $1 \%$, $25 \%, 50 \%, 75 \%$, and $100 \%$. Figure 69, Figure 71 and Figure 73 present the same results but with different scales on the axes to make the comparison with the communications mission's results easier. It is actually very interesting to see how different the utility-cost figures for the communications and the navigation mission are especially in terms of utility. Again, the relative costs do not take into account any learning effects for the traditional spacecraft.

First, Figure 70 shows the architectures utility versus their initial cost for $s=30 \%$ and 25 units produced. The utility of all the fractionated architectures is higher that the utility of the traditional one and the cost of the least fractionated architectures is lower than the traditional architecture's cost. Utility initially increases with fractionation and so does the initial cost of the fractionated architectures, but then decreases while the cost keeps on 
increasing. The traditional architecture's utility is 0.65 . Architectures $\mathrm{B}$ and $\mathrm{C}$ have a higher utility of 0.73 and 15 to $20 \%$ lower initial cost. The least fractionated architectures $\mathrm{D}, \mathrm{E}$ and $\mathrm{F}$, have utilities above 0.9 and still lower initial costs. But for these 3 architectures as the fractionation of the power subsystem increases from 0 to $100 \%$, utility decreases from more than 0.95 to less than 0.85 , and the initial cost increases from $10 \%$ cheaper then the traditional architecture to $30 \%$ more expensive. There is finally an increase in cost and decrease in utility with the fractionation of the propulsion and the ADCS subsystems. The difference in utility and cost is rather small for the architectures with the smallest number of infrastructure modules - architectures $\mathrm{G}, \mathrm{H}$ and I - but is much more accentuated for the architectures with more infrastructure modules architectures $\mathrm{J}, \mathrm{K}$ and $\mathrm{L}$-. The most fractionated architectures costs 28 to $45 \%$ more than the traditional architecture and their utility varies between 0.87 and 0.92 . Cost tend to increase with the number of infrastructure modules but there is no clear relationship between utility and number of infrastructure modules, especially when looking at different overall fractionation levels. Architecture D, which has less infrastructure modules than architectures $\mathrm{E}$ and $\mathrm{F}$, tend to have a lower utility than these architectures, whereas architecture $\mathrm{L}$ which has more infrastructure modules than architectures $\mathrm{G}$ to $\mathrm{K}$ has a lower utility.

Then, Figure 72 shows the architectures utility versus their initial cost for $s=30 \%$ and 100 units produced. When compared with Figure 70, this figure clearly shows the benefits of increasing the number of modules produced: the relative cost of all the architectures decreases and their utility increases. This result contrasts with the results of the communications mission example in which utility did not change with the number of units produced. With 100 units produced and $s=30 \%$, the most fractionated, and therefore more expensive, architectures are less than $20 \%$ more expensive than the traditional architecture and have a much higher utility.

Finally, Figure 74 shows the architectures utility versus their initial cost for $s=0 \%$ and 100 units produced. As $s$ decreases, the utility of all the fractionated architectures increases and their cost decreases and both variations tend to affect more the most fractionated architectures than the least fractionated ones. Architectures $\mathrm{B}$ and $\mathrm{C}$ have a utility of 0.74 and are $30 \%$ cheaper than the traditional spacecraft. Architectures D to L all have a utility higher than 0.9 and the most fractionated architectures are less than $10 \%$ more expensive than the traditional spacecraft. 


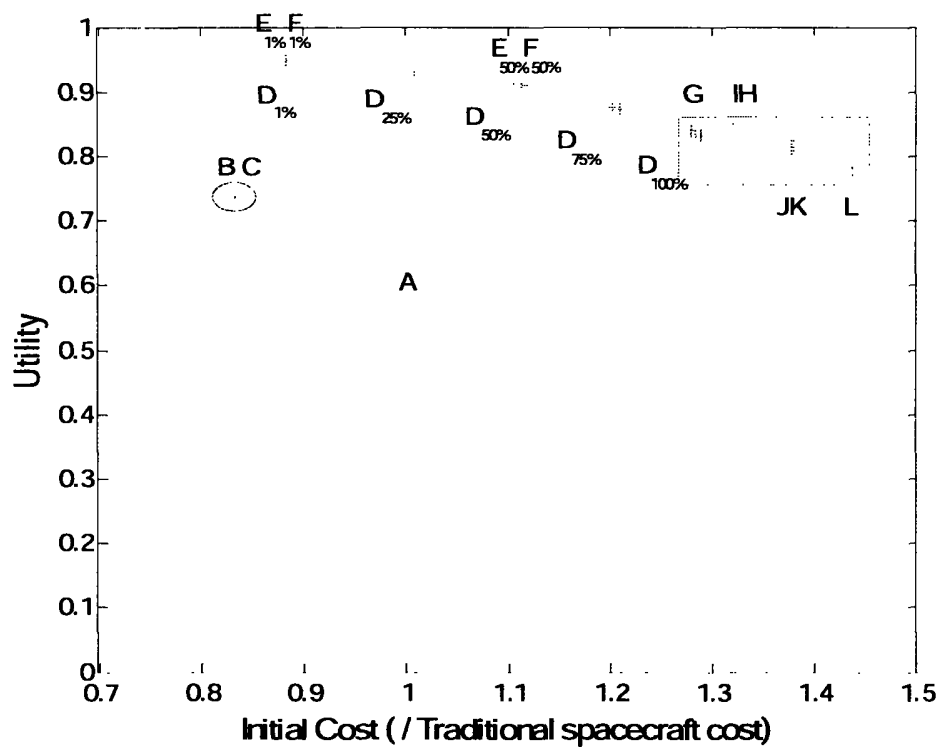

Figure 69: Utility versus initial cost for $s=30 \%$ and 25 units produced

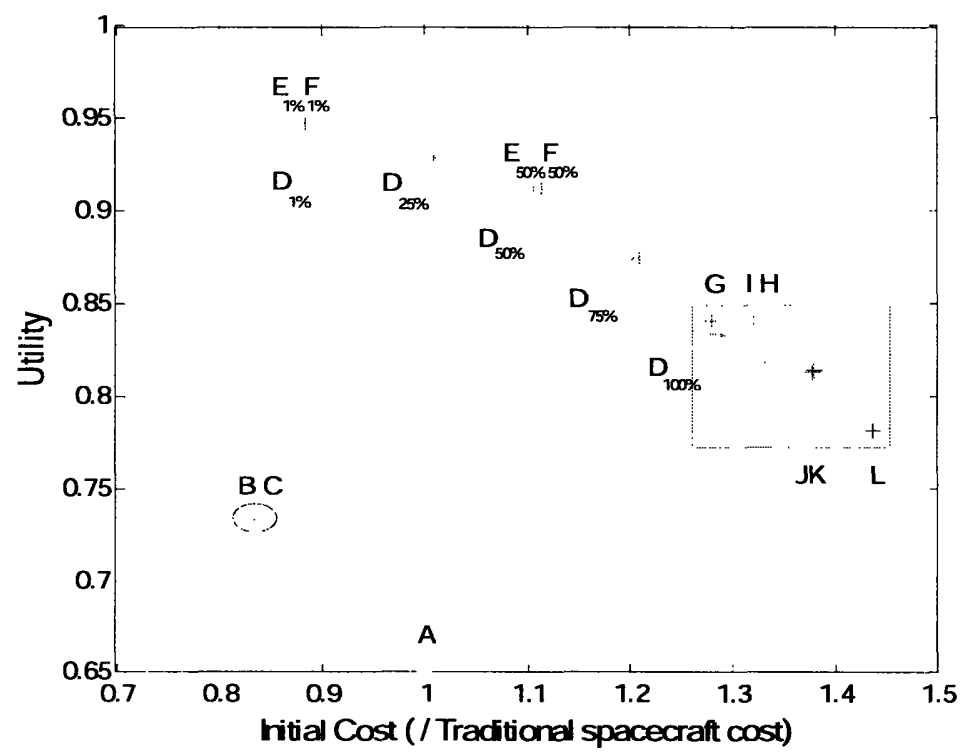

Figure 70: Utility versus initial cost for $s=30 \%$ and 25 units produced (zoom in) 


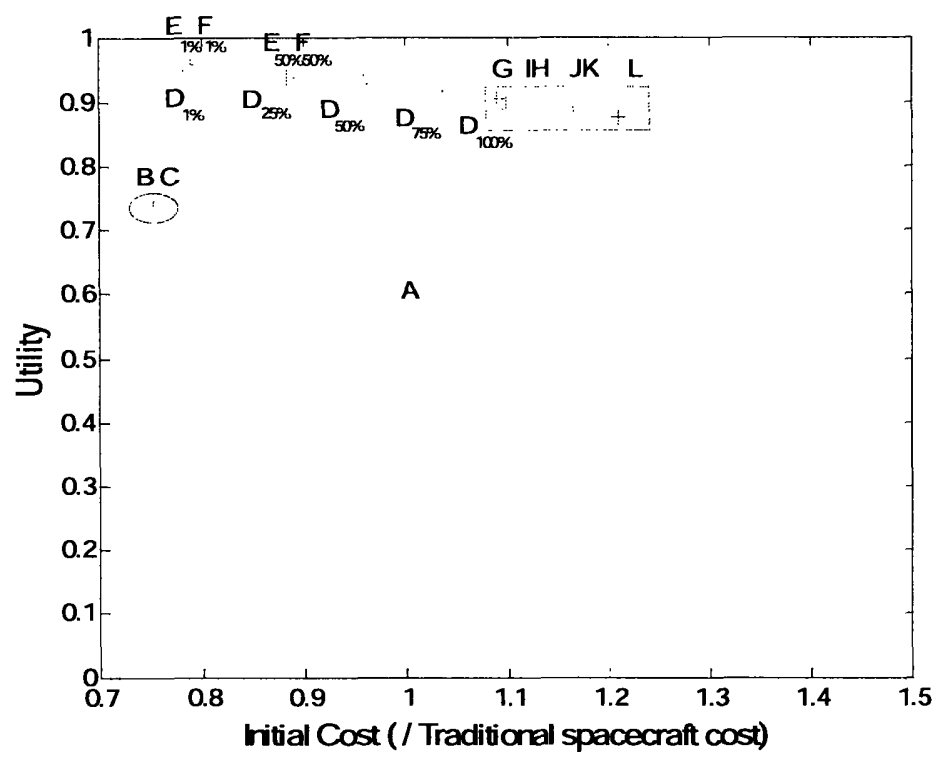

Figure 71: Utility versus initial cost for $s=30 \%$ and 100 units produced

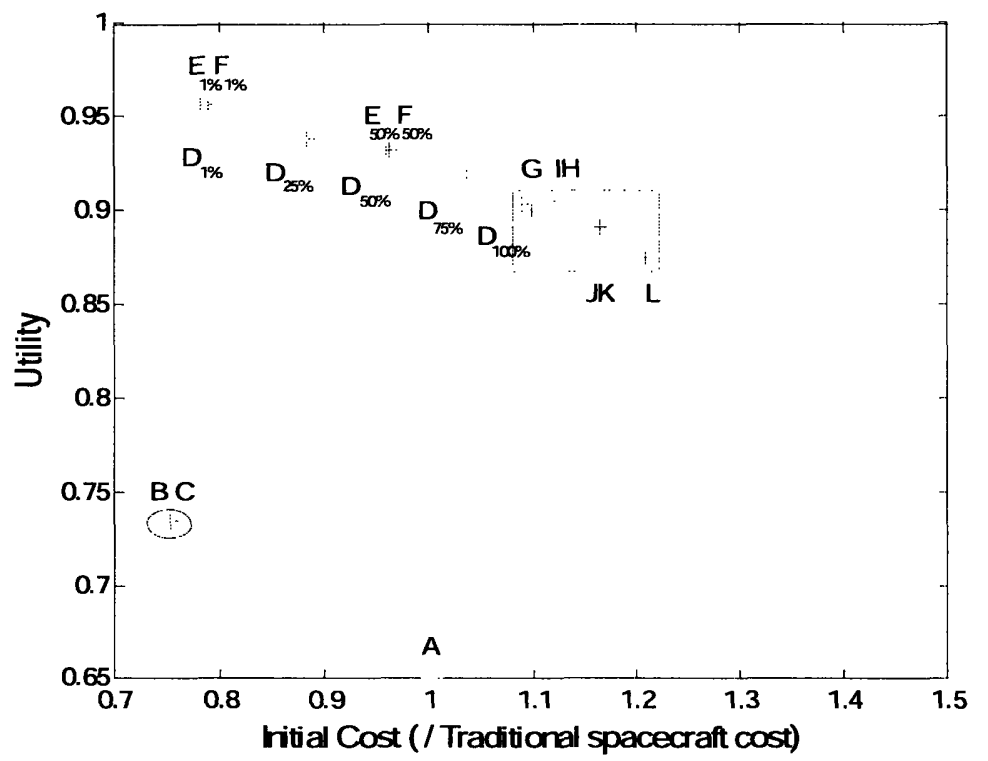

Figure 72: Utility versus initial cost for $s=30 \%$ and 100 units produced (zoom in) 


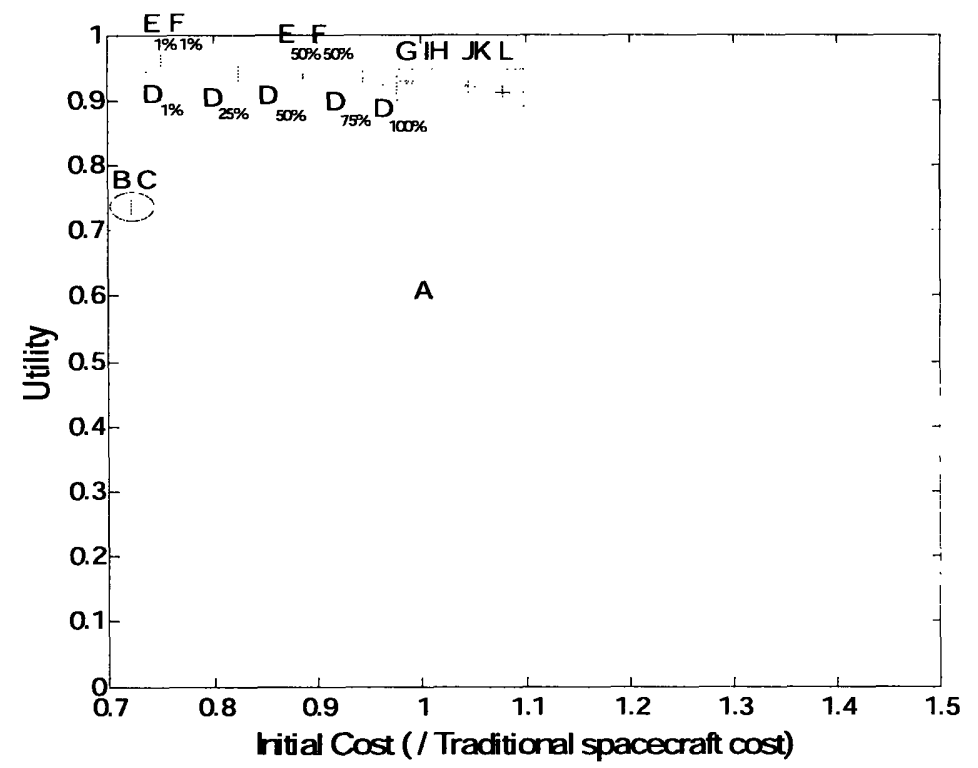

Figure 73: Utility versus initial cost for $s=0 \%$ and 100 units produced

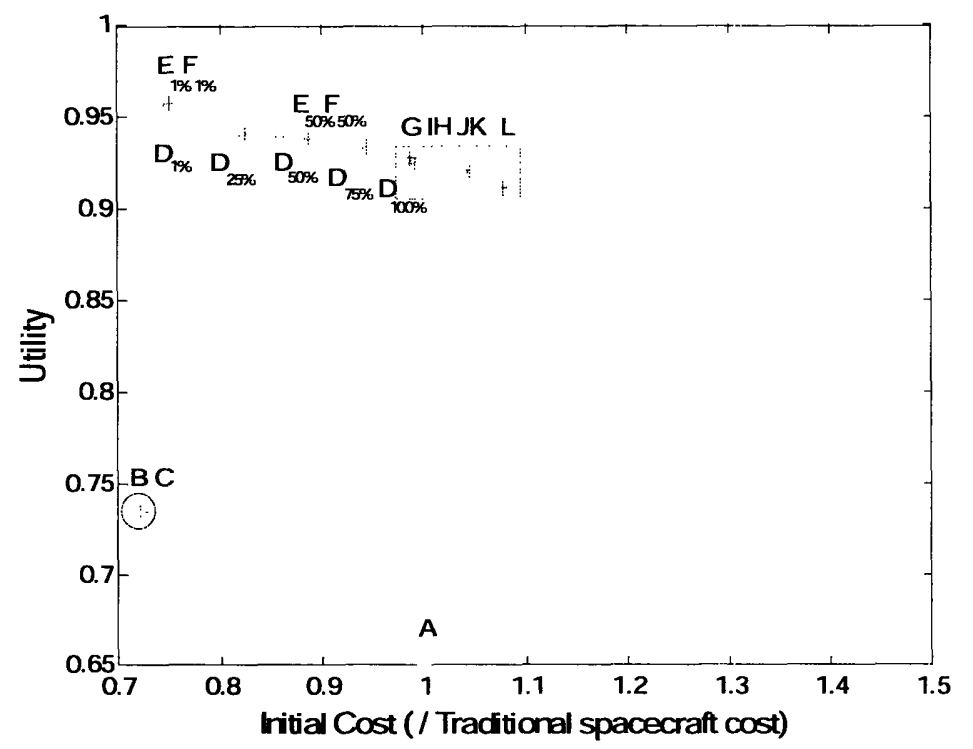

Figure 74: Utility versus initial cost for $s=0 \%$ and 100 units produced (zoom in)

There are eight main conclusions to this analysis of a navigation mission at infrastructure level:

1) There is a significant increase in utility associated with fractionation (if fractionated and traditional architectures are compared)

2) Utility initially increases with fractionation but then decreases when the power subsystem gets fractionated

3) The more fractionated the architecture is, the more expensive it is 
4) Depending on the number of units produced and the standardization parameter, the least fractionated architectures can be cheaper than the traditional one

5) At a given fractionation level, the utility of all the fractionated architectures varies and depending on the fractionation level, the architectures with the largest number of modules may have a higher or a lower utility than the others, but they tend to be the most expensive

6) For this mission, customers are likely to choose some of the least fractionated architectures. Architectures D, E, or F seem to be the "best" fractionated architectures for this mission. They have the highest utility, and even though they are not the cheapest ones, the increase in utility is such compared to the cheapest architectures - architectures B and $\mathrm{C}$ - that they appear as the most promising candidates

7) As the number of units produced increases, costs decrease and utilities increase. When the analysis was done at spacecraft level, fractionated spacecraft did not appear as viable alternatives to traditional ones for navigation missions. But if an infrastructure is developed, fractionated spacecraft may become a viable alternative.

8) Both cost and utility increase with the standardization parameter

\section{F. Conclusions of the Architectural Analysis}

\section{F.1 Conclusions of the analysis of fractionated architectures in terms of cost and utility}

First conclusions can be drawn at both spacecraft and infrastructure levels for all missions. At a given performance level, utility significantly increases with fractionation, i.e. the overall utility of fractionated architectures is always higher than the utility of equivalent traditional architectures. However, utility does not always with the level of fractionation, i.e. from the least to the most fractionated architectures. Then, the initial cost of the fractionated architectures increases with fractionation and most of the time with the number of modules. At spacecraft level, the fractionated architectures are always more expensive than traditional ones, but at infrastructure level, depending on the infrastructure design parameters, some, or even all, fractionated architectures might be cheaper than the traditional architecture. Thus fractionated architectures can dominate traditional ones in terms of both utility and costs at a given performance level.

Then, there are conclusions that are specific to the missions. For communications missions, utility increases with fractionation and is the same for all the architectures with a same fractionation level. However costs increase with both fractionation and the number of modules, so that, at a given fractionation level, and therefore at a given utility level, the architecture with a single infrastructure module is the cheapest one and therefore the best one. Architectures B, D, and G are the best architectures for these missions. For navigation missions, the first conclusion is that fractionated spacecraft may be a worthwhile alternative to traditional ones only if the infrastructure is developed. In addition, the utility of the different architectures for this type of mission is mostly driven 
by maintainability attributes. Utility increases initially with fractionation but then decreases when the power subsystem gets fractionated. Architectures D, E, and F seem to be the best architectures for this type of mission.

This architectural analysis proves that at a given performance level fractionated spacecraft may deliver more value to potential customers and even be cheaper than traditional ones. This demonstrates that if non-traditional attributes are valued enough, customers would choose fractionated spacecraft over traditional ones.

\section{F.2 Other benefits and drawbacks of spacecraft fractionation}

The architectural analysis presented in this first part compared traditional and fractionated architectures at a given level of performance in terms of cost and value derived by potential customers. To complete this assessment of the fractionation concept from a customer standpoint, all the other benefits and the drawbacks associated with the development of an infrastructure of standardized modules are analyzed in this last section.

\section{F.2.1 Potential benefits of fractionation for customers}

Fractionated spacecraft could offer to their potential customers many advantages over traditional ones. All space systems customers could derive more value from fractionated spacecraft than from traditional ones: providers and users, whether they are commercial, governmental, civil and military customers. The potential benefits of fractionation are articulated in this section around four metrics of interest to decision-makers: performance, costs, schedule, and long-term benefits.

\section{F.2.1.1 Potential benefits in terms of performance}

First of all, fractionation could improve spacecraft performance. From a traditional perspective on performance, while traditional spacecraft tend to suffer from obsolescence even before their launch, fractionation enables designers to use the latest technologies because of shorter cycles. Then, spacecraft lifetime is no longer driven by one subsystem. Each module can have its own lifetime, so that the lifetime of each module can be optimized. Moreover, more innovation might be expected, especially in the infrastructure modules, because of the more dynamic market structure associated with modularity. Finally, the standardized modules might to be more reliable than traditional spacecraft bus subsystems. From a non-traditional perspective on performance (i.e. in terms of "ilities"), fractionation might allow customers to better cope with uncertainty during the spacecraft lifetime, as demonstrated above.

In addition, fractionation could make new missions possible, mainly because it "breaks down" large spacecraft into smaller modules. Monolithic spacecraft that cannot be launched today because they are too large or too heavy for the existing launchers could be possibly launched if they were fractionated.

Finally, fractionation could allow designers and customers to make new trade-offs. New trade-offs could be made for instance between the maintainability and the reliability of the different bus subsystems, because fractionated spacecraft are cheaper to maintain. The more reliable a subsystem is, the more expensive it is. Instead of building very reliable systems as they are doing today, designers could choose to build systems with 
fewer redundancies as fractionated architecture would be cheaper to maintain and these systems would initially be less expensive. Furthermore, because fractionated spacecraft are more scalable than traditional ones, it may give customers the possibility to deploy incrementally their space system according to the demand, rather than deploying it all at one time as monolithic architectures force them to do.

\section{F.2.1.2 Potential benefits in terms of costs}

As demonstrated above, the development of an infrastructure of standardized modules could make fractionated spacecraft cheaper than traditional ones, because of learning effects and of the amortization of the development costs over several missions. Traditional customized architectures have large unique development costs and lead to cost inefficiencies because of today's protoflight approach. In addition, large efforts are required to integrate all the subsystems together into a single monolithic spacecraft. On the other hand, the development costs of the fractionated spacecraft modules would be amortized over several missions, and once these modules developed, the unique development effort would be limited to the payload module development. As a result, more money could be invested in the payload.

One issue that was addressed in the architectural analysis is the fact less redundancy may be needed, as both modularity and the infrastructure offer new forms of reliability. Thus fractionated spacecraft could be even cheaper than what was presented above.

Finally, one could imagine that commercial providers could pay only a marginal costs to use the infrastructure, so that the development of the infrastructure would dramatically decrease commercial missions cost. To better assess the impact of fractionation on a mission costs, Figure 75 shows the typical breakdown of a NASA mission lifecycle cost. The space system itself and its launch, which corresponds to the cost figures presented in Part I, represent almost $90 \%$ of the total mission cost. This emphasizes the huge impact the infrastructure would have on commercial missions' costs.

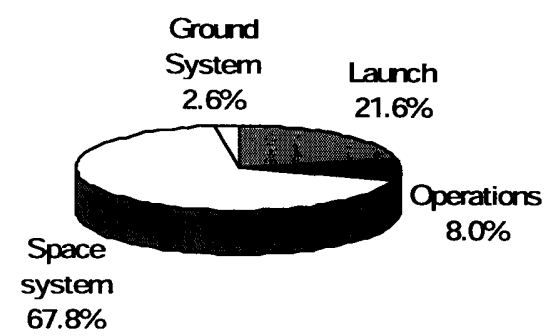

Figure 75: Average NASA Small Spacecraft Mission Cost Breakdown (Sarsfield, 1998)

Finally, fractionation might reduce the high financial risks associated with today's customized monolithic spacecraft. Fractionated architectures significantly reduce the risks associated with the single launch of a monolithic spacecraft, as the different modules can be launched separately (Brown, 2005). The more fractionated the spacecraft is, the lower the risks of launching it might be.

\section{F.2.1.3 Potential benefits in terms of schedule}

Fractionated architectures modularity could make the development, the manufacturing and even the launch of the different subsystems independent from each other. Once the infrastructure developed, the schedule of a mission would be only driven by the 
development of the payload module. The first consequence is that the time to deliver the space-based capability to the customer might be shorter than with traditional spacecraft. Moreover, fewer and shorter delays in the schedule could be expected with fractionated spacecraft as the schedule would depend on fewer subsystems.

\section{F.2.1.4 Long-term benefits}

From a longer-term perspective, spacecraft fractionation may create a flexible, evolvable and scaleable system-of-systems infrastructure that would enable sustainable future space utilization. As highlighted by the comparison with the highway transportation system in (Hastings et al., 2001), today's space enterprise is not based on an approach that is far from being sustainable. Most space systems are unique, designed for the longest life possible, and abandoned when they fail or run out of fuel. Developing an infrastructure of standardized modules might enable to design modules with more optimized lifetimes, to reuse modules for different missions, and to replace failing subsystems.

Furthermore, the development of an infrastructure could allow a cheaper access to space, and therefore create new opportunities. If commercial customers only have to pay a marginal to use the infrastructure, space may become accessible to new customers.

\section{F.2.2 Possible synergies}

In addition to all the benefits mentioned above, some synergies might exist between the concept of spacecraft fractionation and other concepts, which could improve even more space utilization sustainability. First, heterogeneous fractionation could be combined with homogenous fractionation to provide customers with even more flexible, scalable, maintainable, responsive, and survivable space systems. Instead of having a given supporting function in a single module, this function could be provided by several smaller modules. For instance, a payload module could be supported by several smaller power modules rather by a large single one. Second, fractionated architectures could benefit more than traditional architectures from current on-orbit servicing concepts and provide customers with even more maintainable and responsive space systems. Finally, synergies may exist with other concepts of on-orbit infrastructure that would offer a centralized function, such as space solar power platforms.

\section{F.2.3 Drawbacks and risks associated with fractionation}

Fractionation may have many benefits, but it might also have important drawbacks that are inherent to modular architectures and the development of an infrastructure of standardized modules. The potential drawbacks and risks of fractionation are articulated around four metrics of interest to decision-makers: performance, costs, complexity of the operations, and risks.

\section{F.2.3.1 Drawbacks of fractionation in terms of performance}

First, the additional interfaces necessary to the modular architecture is non-productive weight, as it does not in itself add to the functionality of the spacecraft. Then, the standardization of the modules makes their design sub-optimal. As a result, for similar technologies and a given performance level, fractionated architectures are likely to be heavier then traditional ones. 


\section{F.2.3.2 Drawbacks of fractionation in terms of costs}

Even though fractionation may decrease spacecraft costs, it may significantly modify the investment distribution over time. The infrastructure development and investment costs would certainly be larger than non-recurring costs of traditional spacecraft as standardized modular architectures require a larger initial effort to define the standard modules and their interfaces. In addition, large upfront investments are necessary, to develop and bring to maturity the enabling technologies and to develop the infrastructure. However, as mentioned above, these large infrastructure costs could be amortized over several missions.

\section{F.2.3.3 Operations and management of the infrastructure}

Operating fractionated spacecraft may probably be more complex that operating traditional spacecraft, especially because of the number of modules, but one can expect these operations to be more "standardized" than they are today, because of the development of the infrastructure. But a management of the infrastructure itself might probably be necessary in addition to the operations of each mission. In addition, fractionated architectures have a major drawback as they risk generating more space debris than traditional architectures. However, if an infrastructure is developed, its management may include a management of the debris that is better than the current one.

\section{F.2.3.4 Risks associated with fractionation}

In terms of risks, there are obviously technical risks associated with any new and ambitious architectural concept and the new technologies it would use. There might be also an increased risk of collision, as an infrastructure of fractionated spacecraft means that more modules would be on-orbit and that some of these would fly really close to each other to function as a system. Moreover, modules could be exchanged, added or subtracted. The proximity of the modules and their complex operations clearly increases the risk of accident. More modules also generate more risks related to space debris. Finally, there are two other types of risks that can be associated with fractionated architectures. First, there is a risk of technological lock-in and obsolescence associated with the architecture and the interfaces. The modules could evolve in a faster and cheaper manner than traditional spacecraft, but the overall architecture and the standard interfaces could not. Second, there may be risks of downtime associated with the dependency on a single design for the infrastructure modules. If a serious failure occurs with a standardized infrastructure module, as all systems rely on similar modules, other failures of other systems could follow and the production and launch of new spacecraft may be stopped until the problem is solved.

Mainly because of these risks, the overall level of performance of fractionated spacecraft is today perceived by customers as lower than the one of traditional spacecraft. It is interesting at this point to have a look at general innovation theories. According to Utterback, products associated with disruptive technologies tend to initially have a lower performance level than the existing ones that they will eventually replace, as can be seen on Figure 76 (Utterback, 1996). The invasion of the market by the new product is particularly successful if the dominant design of the established product already meets, or even overshoots, customers' needs. This is the reason why when assessing the potential 
impact of an innovation like fractionation, the new architecture and its performance should be compared not only to the existing architecture, but also to the market (Christensen, 1997). The spacecraft built today clearly meet the needs of their customers and most of them tend to live longer then their expected lifetime. Therefore the lower performance level of the fractionated spacecraft is not such large obstacle to its implementation.

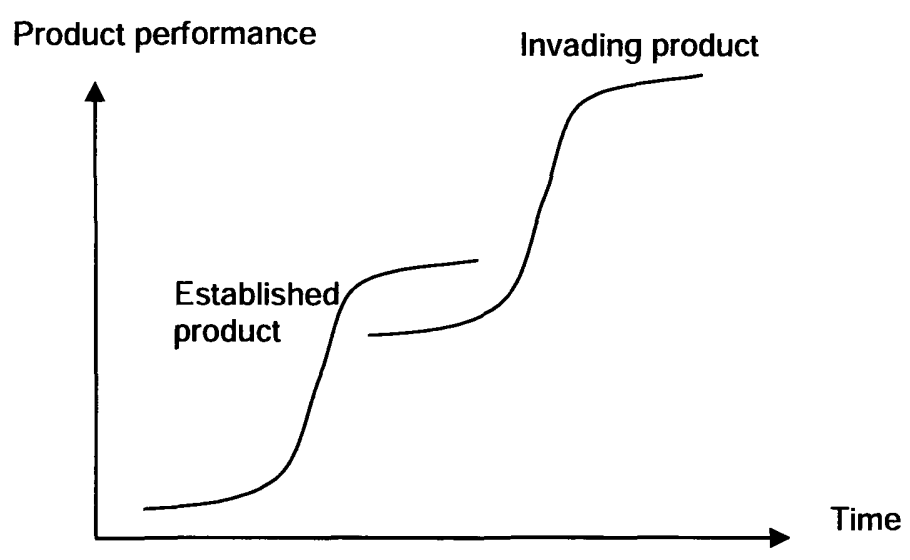

Figure 76: General pattern of invasion process 


\section{Part II - Industrial and Policy Analysis}

\section{A. Introduction to the Industrial and Policy Analysis}

The first part of this thesis demonstrated that fractionated architectures can deliver more value to their customers than traditional ones. This second part investigates the reasons why fractionated spacecraft, which seem to have many advantages over traditional ones, are not implemented yet and are unlikely to be implemented without intervention.

Chapter B examines the changes brought by fractionation on the different spacecraft lifecycle phases. Fractionation actually creates a whole new paradigm, in which spacecraft are no longer customized systems that are built in a prototype approach, but standardized systems built in a mass-production approach.

Chapter $\mathrm{C}$ investigates the consequences of this new paradigm on space industry. Both modularization and standardization have dramatic consequences for all the space industry parties. The impact of fractionation on the industrial organization and the role of each player in this new paradigm are examined. The costs and benefits of the paradigm transition for each of them and for potential new players are assessed. This chapter explains why today's system integrators are likely to resist the implementation of fractionated architectures.

Chapter D examines the other barriers to the development of an infrastructure of fractionated modules. These barriers are managerial, financial or regulatory and require the intervention of a non-market player. The government has the ability to lower these barriers but, as the first customer of the infrastructure, also has an interest in doing it. In Chapter E, the options the government has are explored and specific policies are recommended in order to enable the transition from today's paradigm to a new fractionated spacecraft paradigm. These barriers are investigated in terms of their impact on the level of motivation and ability of all the industry players. Then, the rationales for government intervention are given, and finally recommendations are made that address each of these barriers in order to increase the ability and motivation of all the players to implement fractionated architectures.

\section{B. A New Fractionated Spacecraft Paradigm}

So far in this thesis the concept of fractionated spacecraft has been investigated mostly from a customer perspective. The architectural analysis showed how fractionation provides potential customers with more value and new perspectives on their space capabilities. But fractionation is also associated with changes in all the earlier phases of the spacecraft lifecycle. Fractionation actually creates a whole new industrial paradigm. In this chapter, the changes in both the development and the manufacturing and integration approaches are presented, and today's paradigm is compared with the new fractionated spacecraft paradigm. 


\section{B.1 A new development approach}

In contrast with traditional spacecraft, fractionated spacecraft would have modular and standardized designs. First, interfaces and infrastructure modules would be standardized. Their design would therefore not be optimal for each mission and a large initial effort would be required to develop the modules and the interface hardware. But, as they would be interoperable, the infrastructure modules could be used with different payload modules for different missions. Each spacecraft would require a minimal unique design effort focused on the value-added part of the system, i.e. on the payload. Second, the modularity of fractionated architectures entails a partitioning of the functions in the different modules. Hence each module could be developed and improved independently from the others. Moreover, more recent technologies could be integrated in the modules because of the shorter cycles. Finally, because of the smaller size of the modules compared to the size of equivalent monolithic spacecraft, the design of the system would be less constrained by the launcher size and performance.

\section{B.2 A new manufacturing and IAT approach}

Fractionation would make a modular manufacturing and Integration, Assembly and Test (IAT) possible and the development of an infrastructure of standardized modules could enable a mass-production approach. All the modules could be manufactured, assembled, and tested in parallel, independently from each others. The manufacturing and IAT processes of the infrastructure modules could be standardized and automated. Tests would be simpler, and the assembly and test sequences of the infrastructure modules would no longer need to be designed for each mission. In case of default, other similar modules could be used as spares, and the access to the components would be much easier. Manufacturing and IAT cycles would be much shorter than with traditional spacecraft. However, the systems could not be fully tested before launch, especially when modules would be exchanged on-orbit.

\section{B.3 Synthesis of both paradigms}

Figure 77 summarizes and compares today's paradigm and of the new spacecraft paradigm. The comparison is done for the main spacecraft lifecycle phases and for the main characteristics of the mission. 


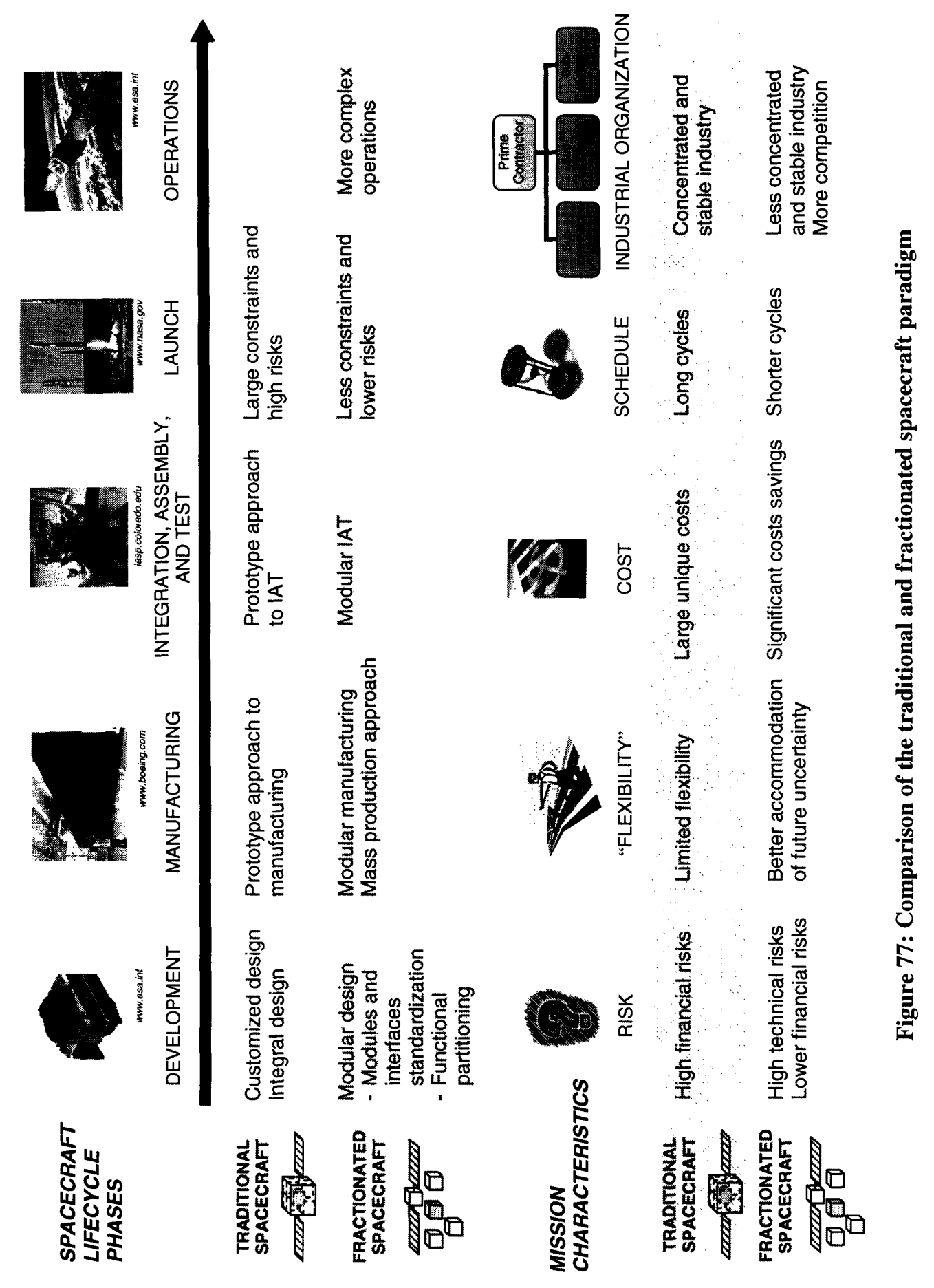




\section{Impact of Spacecraft Fractionation on the Space Industry}

Fractionation represents a radical change in spacecraft architecture that would be associated with drastic changes in the industrial paradigm. This change in industrial paradigm would be coupled with transformations in the industrial organization as shown on Figure 78. This chapter investigates the impact of fractionation on the industry and its players.

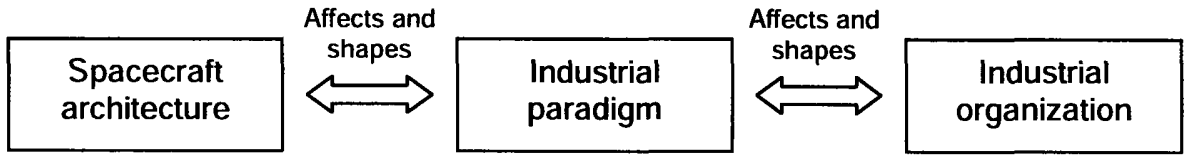

Figure 78: Impact of fractionation on the industry

The main driver of the changes in the industrial organization is modularity. A modular architecture made of smaller modules could actually prompt sweeping changes to the space industrial base structure. The industry would gain new dynamics, become much more competitive, and much less stable and concentrated, as described in (Baldwin and Clark, 2000). The shorter cycles would set a faster pace and new opportunities would be created more frequently. However, modularity would not have such a large impact across the aerospace industry, which today produces customized, one-time use products, if the infrastructure is not developed. The infrastructure development and maintenance requires a significant volume of production of identical modules that would transform today's protoflight approach into a mass-production approach, and only in this mass-production approach would modularity have a major effect on the space industry.

\section{C.1 Change in the space industry organization}

\section{C.1.1 Today's spacecraft manufacturing industry}

\section{C.1.1.1 A particular industry}

Satellites are large and complex systems that are customized for each mission and made of tailored components. Their architecture has evolved over time, but today manufacturers have adopted a common dominant design. In today's paradigm, spacecraft are associated with very long cycles, high costs and high risks. All these product and production characteristics led to an atypical industry structure.

The satellite market, whether commercial or governmental, represents a rather small market in terms of number of satellites delivered each year. Moreover, it has high barriers to entry: first, because of the current excess capacity of the industry but also because of the high costs and risks, the large infrastructure necessary, and the low marginal profits. As a result, the satellite industry has an oligopoly/oligopsony structure. On the demand side, the main buyer remains the US government, even if there are other commercial buyers. The supply side consists in a few companies as in the past decades the satellite manufacturing industry went through waves of rationalization and mergers. This consolidation led to a very concentrated industrial base, with a couple of contractors and a few small, highly specialized subcontractors. In addition, this market is highly regulated 
and at least two major suppliers are, and will be, maintained for security reasons. Furthermore, the products are mostly determined by the customers and prices are negotiated, so that both products and prices are determined only to a limited extent by market mechanisms. Finally, risks are often shared between the government and the firms. Besides, the establishment of a dominant spacecraft design has led to the development of synergies that are specific to it. These specific synergies, which affect skills, production systems, and cognitive systems, have stabilized the industry, and are sources of rigidities in the evolution towards any new architecture (Cebon et al., 2002). As a result, the organization of today's satellite manufacturing industry is very stable.

An interesting feature of this industry, and of other similar complex systems industries, is the large involvement of the users in any innovation process (Hobday, 1996). Fractionation is a good illustration of this phenomenon, as it is an innovation that originated from and is supported by a group of users. More generally there are rather intimate users-producers links, and users tend to be involved in all the satellite development and manufacturing phases.

\section{C.1.1.2 Role of the different players}

Today's satellites industry consists of two main types of players: a few large suppliers and a few small subcontractors. The large suppliers are mainly system integrators, and the highly specialized subcontractors provide them with the different subsystems. As shown in Figure 79, the relationships between these two types of players are vertical and hierarchical, and the interactions between the sub-contractors are very limited. Different integrators can share common sub-contractors.

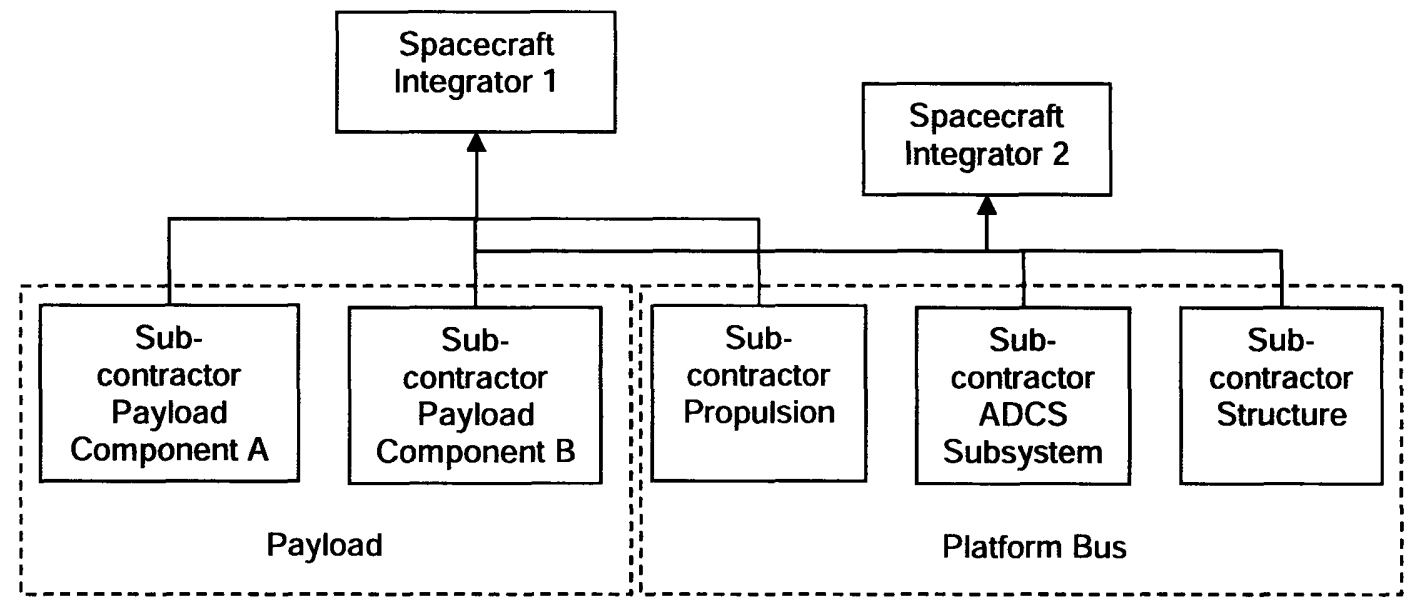

Figure 79: Today's satellite manufacturing industry

The system integrators deal with the customers and manage the overall system. They design the satellites and define the system requirements and provide them to the different sub-contractors. The system integrators closely follow the development, manufacturing, and test of all the subsystems by the subcontractors. Finally, they integrate, assemble, and test the whole system. Systems integration is a high value-added activity, and the systems integrator role is perceived as a highly profitable and desirable one to assume and protect (Chen, 2001). In contrast, the subcontractors are typically specialized in a single 
component or subsystem. They design their components or subsystems according to the requirements they are given by the integrators.

\section{C.1.2 The industrial organization associated with the new paradigm}

\section{C.1.2.1 A new industrial organization}

The new paradigm created by fractionation would transform the current industrial organization. The number of infrastructure standardized modules to be produced is assumed to be large enough so that their manufacturing has transitioned from a prototype approach to a mass-production approach. As explained in Part I, fractionated spacecraft can have more or less fractionated architectures. Obviously the change in industrial organization would be proportional to the architectures fractionation level. This section will focus on the industrial organization that would correspond to the most fractionated architectures, in which all the "fractionable" functions are fractionated in separate modules. If the architectures of interest are not the most fractionated ones, the new industrial organization is likely to be an intermediary organization between today's organization, presented in the previous section, and the one presented in this section.

Fractionated spacecraft payload modules would remain very expensive customized systems with tailored components. These modules would still be associated with a very concentrated industry structure with a few large integrators. In contrast, the infrastructure modules would become much simpler products centered on a single function. They would be standardized modules with standard components that would be manufactured in a mass-production approach, in large volume, with standardized and automated procedures for manufacturing, assembly, and test.

The different modules that form a fractionated spacecraft would be supplied by different companies, as opposed to today's organization in which a single system integrator provides the whole spacecraft. As shown on Figure 80, the payload module could be supplied by an integrator and each infrastructure module could be supplied by different suppliers.

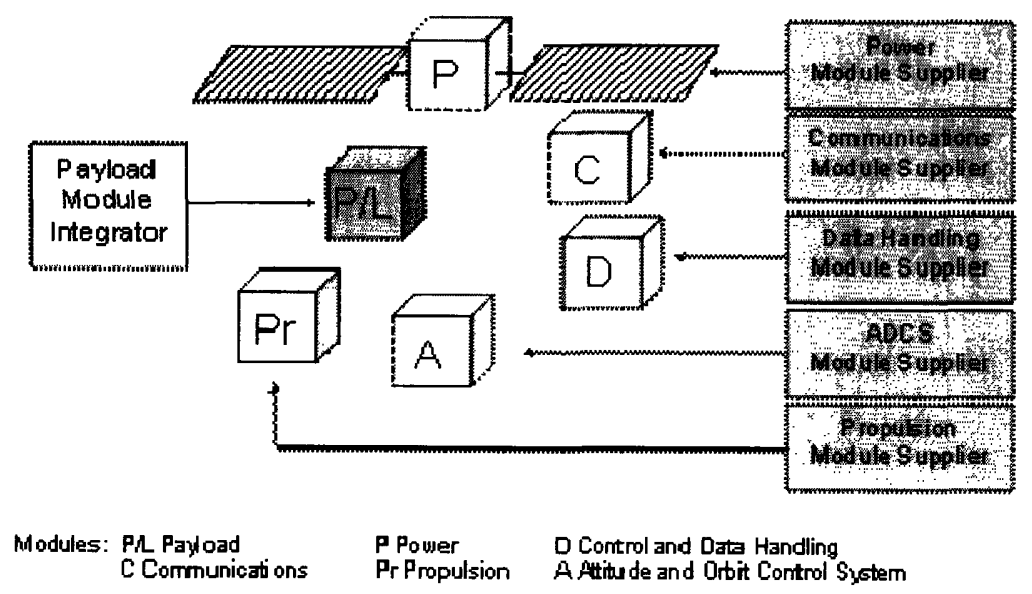

Figure 80: The suppliers of the different fractionated spacecraft modules 
C.1.2.2 New roles for the current players and potential new players

First of all, fractionation would lead to two different types of industrial organization: one for the payload module and another one for the standardized infrastructure modules.

The industrial organization corresponding to the development, manufacturing, integration, and test of the payload module would be quite similar to today's organization, as presented on Figure 81 .

The current system integrators would keep their role of integrator of a complex customized system. They would work with the same subcontractors as today for the payload and the subsystems that are not fractionated (e.g. structure and thermal subsystem). But in addition to these subsystems, new components will have to be integrated in the payload module. These new components are the various interfaces with the infrastructure modules. These interfaces would be standardized like the infrastructure modules and be initially developed based on very specific technologies (power beaming technology, electromagnetic formation flight technology, etc.). Thus the subcontractors that would provide these interfaces would be either current subcontractors - for instance the wireless communications interfaces could be provided by some of the current communications subsystem subcontractors - or new firms that would have invested in a specific technology.

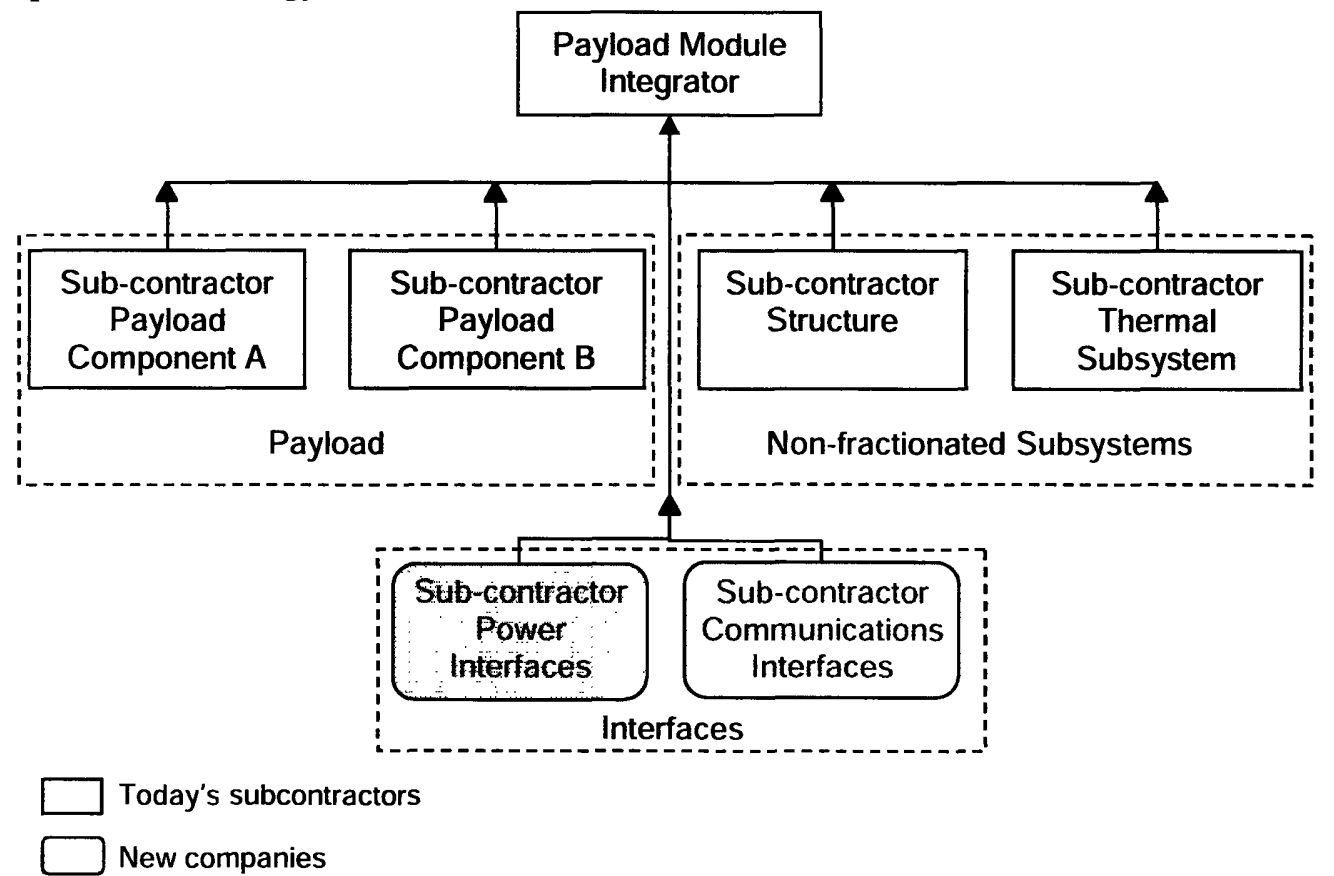

Figure 81: Industrial organization for the payload module

For the standardized infrastructure modules, the industrial organization would be totally different than the current one, as presented on Figure 81. The transition from an integral architecture to a modular one would have a significant impact on the industrial organization as presented in (Baldwin and Clark, 2000). The different bus subsystems would no longer be assembled, integrated, and tested all together. Each module would be developed, manufactured and tested independently, so that there would no longer be the need for an integrator per se for integrating the whole spacecraft. As each module 
corresponds to a supporting function, they would certainly be supplied by the current bus subsystems subcontractors. For instance firms that are providing today power subsystems to the system integrators would take the responsibility for power modules. In addition to the main supporting function they provide to the payload module, these infrastructure modules would include the subsystems that are not fractionated (e.g., structure and thermal subsystems) and the interfaces with the other modules. The subsystems that are not fractionated would be provided directly to the module suppliers by today's subcontractors of these subsystems, and the standard interfaces would be provided by either current subcontractors or new firms like for the payload module.

Once the architecture of the infrastructure modules and the standard interfaces defined today's system integrators may no longer have a significant role to play in the supply of the infrastructure modules, even though a coordination role is still needed. When a product is modularized, there are usually two groups of firms: the ones that specialize in modules and the ones that specialize in aggregating modules and assembling them into final products (Cebon and al., 2002). But as fractionated spacecraft would be modular but not assembled as a single system, there would be no need for firms that would assemble the whole system, even though the coordination function would still be needed. The infrastructure modules could actually be provided to the customers directly by the modules suppliers, i.e. today's subsystems subcontractors. This illustrates the impact of modularity on an industry. A parallel can be drawn here with the computer industry. The evolution of PCs architecture from an integral architecture to a modular architecture led to dramatic changes in the industry organization and put an end to the leadership situation of IBM, which initially had the role of system integrator when computer architectures were integral. Modularity has transformed a highly concentrated industry into a much less concentrated one (Baldwin and Clark, 2004). Furthermore, customers who initially needed to buy an integral system to IBM can today buy computer components directly from smaller suppliers. Some of these suppliers used to be subcontractors of the firms that were selling integral systems, like IBM, and some are new firms that entered the market once the architecture of PCs was modular. This example shows how "modularity is a financial force that can change the structure of an industry" (Baldwin and Clark, 2004).

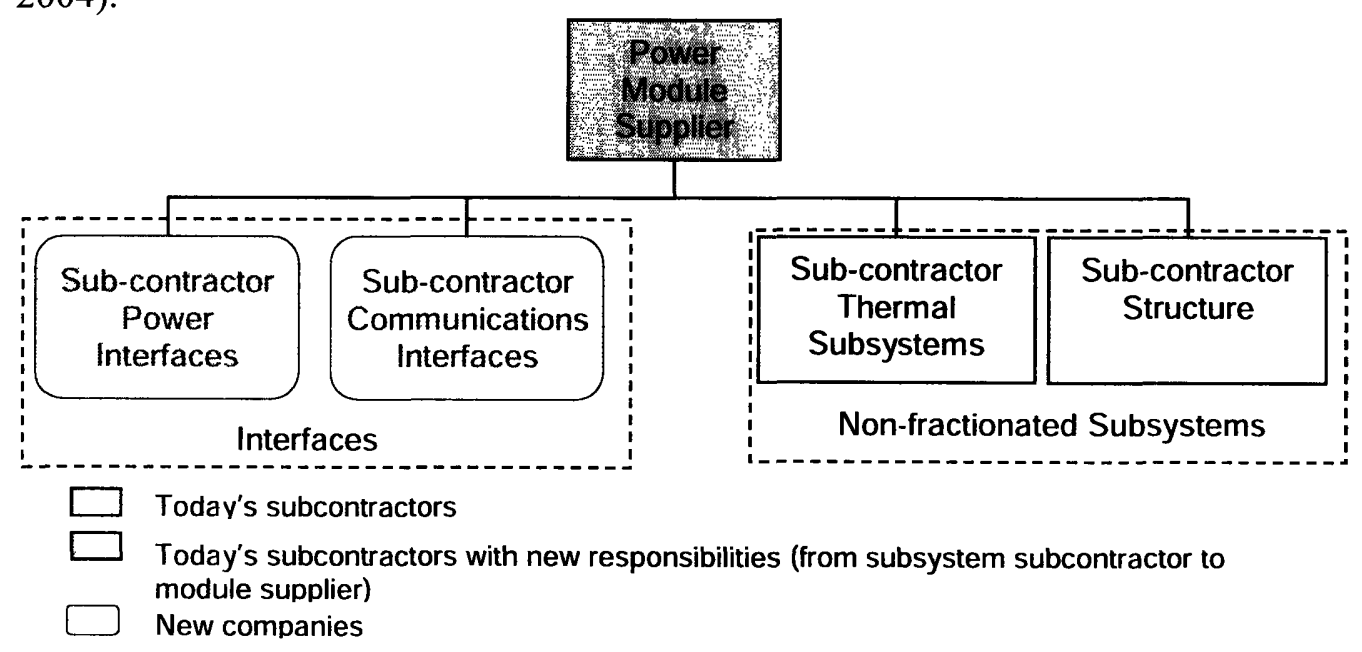

Figure 82: Industrial organization for the standardized infrastructure modules 
Figure 83 summarizes the industrial organization corresponding to the new fractionated spacecraft paradigm. The payload module integrator and the module suppliers would share common subcontractors for the non-fractionated subsystems and for the interfaces.

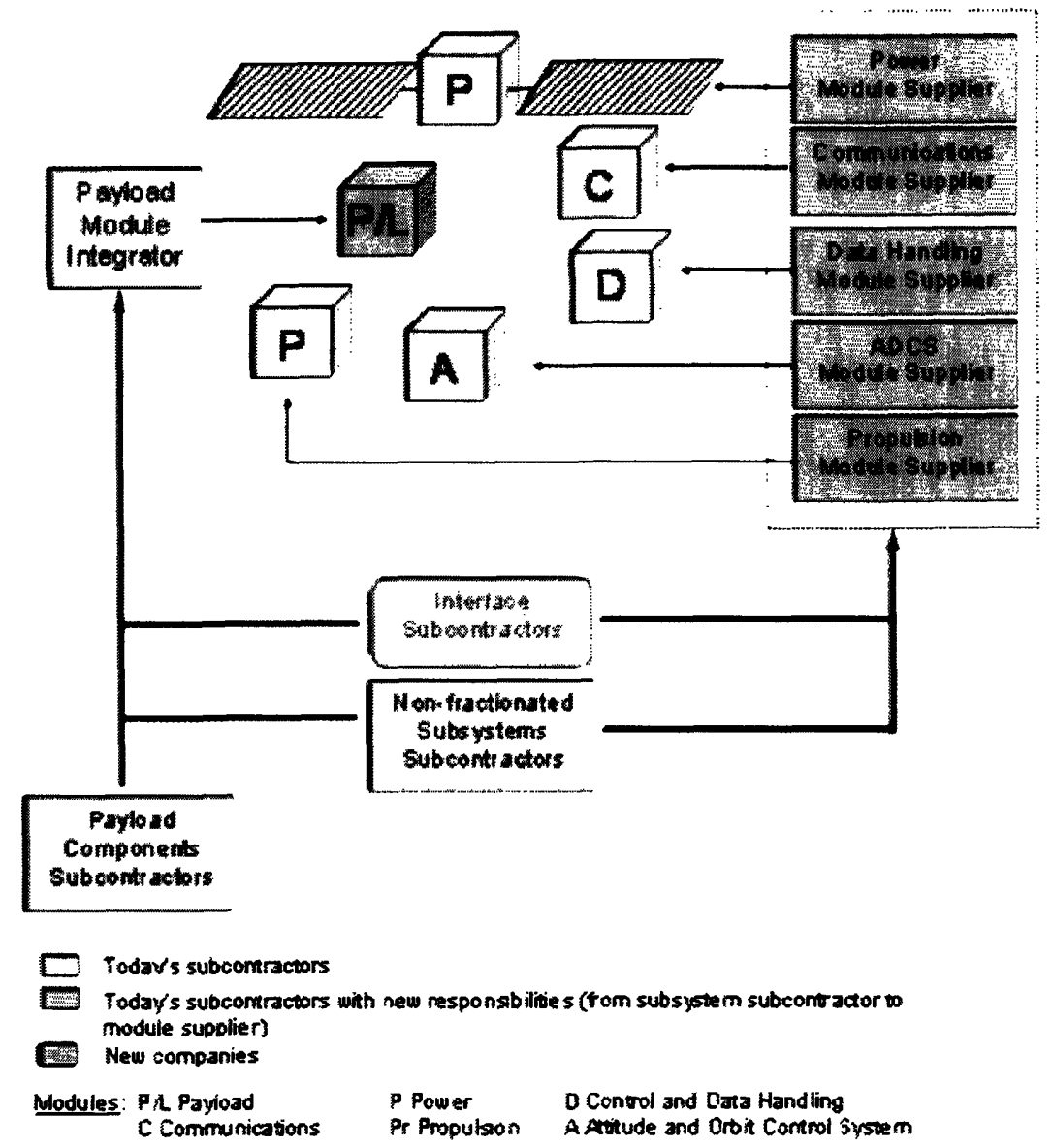

Figure 83: Industrial organization in the new fractionated spacecraft paradigm

Even though system integrators may no longer have a significant role to play as integrators once the architecture of the fractionated spacecraft and its interfaces will be defined, they are likely to have a crucial role to play in the transition period from traditional to fractionated architectures. "Modularizing" a system involves specifying its modules, interfaces, and standard tests for the whole system (Baldwin and Clark, 2004). Because of their expertise and experience with traditional spacecraft, these integrators are actually the firms that are in best position to specify these elements and define the fractionated spacecraft architecture. They would play the role of the architect firm (Frigant, 2004). As a result, today's system integrators could be asked to develop a new spacecraft architecture, but once this architecture would be defined, they would have no role to play in the production of the standardized modules they specified.

Such changes in the industrial organization also mean that if both traditional and fractionated spacecraft are manufactured, two or three separate procurement systems 
would be necessary: for the traditional spacecraft, for the payload modules, and for the standardized infrastructure modules.

In conclusion, if fractionated architectures are implemented, today's integrators would become architect firms and payload module suppliers and today's subcontractors would become infrastructure module suppliers.

\section{2 Consequences of fractionation on the space industry players}

Fractionation would be a disruptive innovation for the industry that would change the role of the current industry players and may attract new players. This section first investigates the costs and benefits of fractionation for the main current players, i.e. the system integrators and their subcontractors. It then analyses the advantages and disadvantages new entrants may have on the current players. Fractionation would be so disruptive because it is unlikely to really broaden the market for satellites and fractionated spacecraft would mainly substitute for traditional ones (Utterback, 1996). This assessment is based on the assumption that fractionated spacecraft would substitute for traditional ones only for a limited number of missions so that both traditional and fractionated spacecraft would be produced in parallel.

To measure the challenge that each player face, the impact of fractionation is measured in terms of its relationship to existing business and technical capabilities (Utterback, 1996). These capabilities include resources (what the firm has), processes (how a firm does its work) and values (what a firm want to do) of the firms (Christensen and al., 2004).

\section{C.2.1 Impact of fractionation on the current players}

The costs and benefits of fractionation for today's industry players are considered in this section in both absolute and relative terms. In fact, the relative costs and benefits determine the new competitive advantages of the players on the spacecraft manufacturing market.

\section{C.2.1.1 Costs and benefits of fractionation for the system integrators}

\section{- Costs}

The system integrators would have many reasons to resist the development of fractionated spacecraft and try to maintain the status quo. First, they would tend to resist fractionation for economic reasons as they have made huge investments in their current facilities and technologies, which may become obsolete with fractionation. They carry the burden of large investments in people, equipment plant, materials, and knowledge, all of which are closely linked to the status quo. It is important to note that whether the system integrators would be able to use their current technical capabilities and how many new capabilities they would need depends on the "size" of the payload modules. As fractionated architectures allow customers to launch larger payloads, payload modules may grow larger than what they are in traditional spacecraft, and system integrators may be able to use their current facilities, or at least part of them, to integrate, assemble and test payload modules. Thus system integrators would not need brand new facilities to integrate and test fractionated spacecraft, and could integrate and test traditional spacecraft and payload modules in the same facilities. Still, fractionation can be 
considered as competence-destroying for the system integrators, as they render obsolete some of the expertise and knowledge associated with the traditional spacecraft architecture (Tushman and Anderson, 1996). Second, emotionally, they and their fortune are associated to a great extent with the status quo. Finally, their managerial attention is encumbered by their current system, and it would be very difficult for them to maintain two types of management at a time if they get involved in the fractionated spacecraft development (Utterback, 1996).

In the transition from traditional to fractionated spacecraft, the industry profits would be redistributed among the industry players, as described by the value chain evolution theory. This theory supports that industry tend to evolve from states of interdependence, where leading firms earn the lion's share of industry profit, to modularity, in which smaller firms responsible for critical components would earn a disproportionate share of the industry profit (Christensen and al., 2004). The system integrators appropriate today most of the value in the spacecraft development and manufacturing value chain. Fractionation would allow the subcontractors that are responsible for critical subsystems (such as the power subsystem), to earn a larger share of the overall profit.

As mentioned above, with fractionated spacecraft, system integrators would loose their role of system integrators and their activities of system integration, assembly and test. Moreover their role as architect firm may be limited to the initial design of the fractionated spacecraft architecture, which would probably be not proprietary as an open architecture would facilitate the infrastructure development.

The position of the system integrators on the market would evolve, so that they would no longer be able to appropriate all the value generated by space systems manufacturing (Chen and Shi, 2001). First, system integrators would not be in a position to influence the modules costs except through their initial architecture. Second, they would no longer be able to control the flow of information concerning the infrastructure modules. The main decisions regarding the development and manufacturing of the spacecraft are today centralized and taken by system integrators. Fractionation would lead to a decentralization of these decisions, as each module can evolve independently once the interfaces are defined. System integrators would loose decisional power, and therefore market power, to the benefit of the module suppliers.

Today's system integrators could retain some of the value in their role of architect firms, i.e. the firms that define the modular architectures. It is unlikely that once the system integrators will have defined an initial fractionated architecture, they will not try to remain involved in the system development and manufacturing. One way for them would be to regularly propose improvements in the fractionated architecture and in the interfaces, and they could try to keep in-house the interfaces development and manufacturing. There are long-term risks associated with this position of architect firm. First of all, architect firms risk to loose technical expertise if most innovations occur at module level. As subsystems are improved by the module suppliers, they may also loose the expertise to evaluate their subcontractor prices for the traditional subsystems they integrate. They would also tend to loose their own capacity to innovate. Finally, the redistribution of decisional power in the industry and the new relationships with the subcontractors - that may become module suppliers - could have negative long-term impact on the architect firms (Frigant, 2004). 


\section{- Benefits}

The main benefit of fractionation for the current system integrators is that it would refocus their activities on the payload, which is the element that creates most of the space systems value. As mentioned above, fractionation would allow customers to launch larger payloads, so that system integrators can reasonably expect to integrate larger payloads, and therefore sell more expensive systems. Larger payload, and therefore large payload modules, would be in their interest also as they would allow them to a certain extent to use their current spacecraft integration, assembly and test facilities.

In addition, system integrators may benefit from the innovations of the module suppliers and therefore integrate cheaper subsystems with improved performance in their traditional spacecraft.

Given the potential costs and benefits, the uncertainties surrounding them, and the limited size of the market for fractionated spacecraft, one can reasonably expect today's system integrators to resist the paradigm shift and try to maintain the status quo.

\section{C.2.1.2 Costs and benefits of integration for subcontractors}

\section{- Costs}

First of all, if they become module suppliers, subcontractors would have to transition from a prototype approach to a mass-production approach they are most likely not familiar with. This transformation would have significant costs for them. Besides, one of the advantages of fractionation is that each module can have its own lifetime, independently from the payload module lifetime. If the module suppliers remain subcontractors of the system integrators for traditional spacecraft subsystems, they may have to manufacture subsystems with different lifetimes. Finally, modularity would increase competition on the market, and this competition would focus on the modules. The subcontractors are likely to be threatened by potential new entrants, and innovations at module level would become essential to succeed in this more competitive environment.

\section{- Benefits}

If they become module suppliers, fractionation can be considered as competenceenhancing for subcontractors. They would have to integrate self-standing systems and develop standard procedures to manufacture and test them. By providing modules, subcontractors would gain more decisional power and more power on the market relative to today's system integrators. They can reasonably expect to capture more profit from these standardized modules than from the provision of subsystems to the system integrators for traditional spacecraft. They would have more control on prices than they have today, despite the increased competition. Concerning the subsystems they will go on providing to the system integrators for traditional spacecraft, they are likely to benefit from their innovations in the modules.

A last consequence for the subcontractors if they become module suppliers concerns human resources. Depending on the perspective, it could be considered as a cost or a benefit of fractionation. In the prototype approach, most of the knowledge is generally embodied in people, whereas in the mass-production approach associated with fractionation and the infrastructure development, the know-how would be embodied 
mostly in the machinery, so that less qualified people would be needed and the loss of an employee would be less critical than it is today (Hobday, 1996).

\section{C.2.2 Potential new players}

By modularizing the spacecraft architecture, one of the major barriers to entry on the market, the high costs of developing an entire complex spacecraft, would be reduced to the costs of developing individual modules (Baldwin and Clark, 2004). New firms may therefore more easily enter the market. New entrants may be interested in three types of activities: first, the supply of entire modules, second, the integration of modules, i.e. system integration activities at module level, or third, the provision of the standard interfaces. Most new entrants would be expected compete on the manufacturing and/or integration of the specialized infrastructure modules.

The main advantage of the industry outsiders over the insiders is that they have little to loose in pursuing innovations such as fractionation. They do not have any existing infrastructure or knowledge to maintain or defend, and have every economic incentive to overturn the existing order (Utterback, 1996). Moreover, new entrants with an experience in mass-production may have a significant advantage over today's subcontractors.

However, some of the characteristics of the space industry may be to their disadvantage. First, this industry is highly regulated and transactions and information exchanges are limited by stringent export control regulations. Furthermore, the administrative burden for any firm involved in space activities is very large. All these characteristics may prevent new entrants that may be used to more open market environments to enter the market. Finally, even if fractionation decreases the investment necessary to enter the spacecraft market, barriers to entry remain high and the market will remain rather limited. High-tech technologies that are often specific to space applications are involved and the current players clearly have a technological advantage for some subsystems or components.

As conclusion, fractionation could prompt sweeping changes in the space industry to which today's large system integrators are likely to resist.

\section{Barriers to Spacecraft Fractionation Implementation}

Despite so many potential benefits for the customers, there are major barriers to the shift from today's paradigm to the new fractionated spacecraft paradigm. The possible resistance of some of the stakeholders presented in the previous chapter is only one of them. There are other important challenges of economic, managerial and regulatory nature, which are examined in this section.

\section{D.1 Development of the enabling technologies}

The first step in the transition from traditional to fractionated spacecraft is to make fractionated architectures technically feasible and viable. There are two types of technologies that need to be developed and brought to maturity in order to make this shift possible. The first type of technologies would make fractionation technically feasible. These technologies correspond to the three critical capabilities identified in Part I. The 
first capability necessary and the one that can be considered today as the most mature of the three, is the wireless data transfer capability. Wireless communications technologies, whether RF or optical, have already been studied, developed, and experimented, and largely benefit from the developments of similar technologies for ground applications. The second capability necessary to make fractionation possible is the power beaming capability. Laser and microwave power transmissions techniques have been studied but are still in an early development phase. Some technologies may use a single laser beam to carry both power and information packets so that power beaming and wireless data transmission could be achieved with a common technology. Finally, the last capability necessary to fractionation is the collaborative separated positioning capability. Technologies that would achieve this capability, such as electromagnetic formation flight technologies, are still in an early development stage. The second type of technologies that should be developed would make fractionation viable. The best example is the technologies associated with the power subsystem. As identified in Part I, the first technological challenge is to reduce power subsystems mass. Without technologies that would make smaller and lighter power subsystems possible at reasonable cost, fractionated spacecraft may never become a viable alternative to traditional ones.

The development of these two types of technologies requires important investments. Some of these technologies, such as wireless communications technologies, may be improved in the close future without any specific investments directly related to fractionation. They are, or will be, also used in traditional spacecraft for different missions, and even on other ground-based systems, therefore private firms already have strong incentives to develop these technologies. But other technologies, such as power beaming technologies, may require specific investments from the government, which is the main customer for fractionated spacecraft. These technologies would otherwise not be developed and hinder the implementation of fractionated architectures. These technologies are not developed today for various reasons. First, they are associated with high costs and risks. Second, the market for these technologies is today limited to very specific applications such as fractionation. The lack of incentives for private firms to develop these technologies must be addressed by determining who should perform these R\&D activities and how they should be funded. Finally, these technological developments should be coordinated in some way with the definition of the fractionated spacecraft architecture, as these technologies and their level of performance will influence the choice of the architecture.

\section{D.2 Development and management of the infrastructure}

The third step after the development of the enabling technologies and the definition of the fractionated spacecraft architecture is the development of the infrastructure. The barriers to this development are both financial and managerial.

\section{D.2.1 Investment in the infrastructure}

First of all, given the risk and costs involved, the infrastructure may be developed only if a minimum future market is guaranteed. Then, developing an infrastructure of standardized modules requires, like any other infrastructure, a huge investment that is unlikely to be borne by the private sector. Moreover, this investment has to be made 
upfront, well before the infrastructure can be used and any potential benefits be derived from it. Finally, this funding has to be stable over a rather long period of time, as the infrastructure development would take a long time.

a. Management of the infrastructure development

The development of the enabling technologies, the definition of the fractionated spacecraft architecture, and the development of the infrastructure would probably be done by different entities, some of which are usually in competition with each other and not allowed to cooperate. Thus, the infrastructure development would be hindered by antitrust regulations and a lack of coordination between the different entities involved, whether these are firms, universities or research laboratories.

To derive maximum value from the infrastructure and reduce the inefficiencies in its development and use, the infrastructure elements should be interoperable. Thus the infrastructure requires common standards, to which network externalities can be associated. The interfaces of the fractionated architectures would be based on new technologies for most of which no standards exist today. This uncertainty about the set of standards that would finally be adopted is another barrier to private investment in the technologies and the infrastructure development. Firms perceive the lack of standards as a major source of uncertainty, and are not willing to enter into a technological trajectory (Dosi, 1988) if their standards risk not to be selected as the infrastructure standards. Without an initial agreement on minimum standards, it is unlikely that firms would invest in developing them.

\section{b. Management of the infrastructure}

Once the infrastructure initially developed, it would have to be managed. The last set of barriers that prevent the transition from traditional to fractionated spacecraft paradigm deals with these managerial issues. A centralized entity would be needed to manage an infrastructure of fractionated modules. Moreover, the management of this long-term program would have to be stable. It is unlikely that private firms would take the lead in such a risky enterprise, at least in the early phases.

The fractionated modules infrastructure would initially be developed for military customers, but there could later be commercial users for this infrastructure or a similar one. Commercial users could access this infrastructure at a marginal cost. This transition would be possible only if the government anticipates it, as it may influence the initial design of the infrastructure. Offering the access to the infrastructure to commercial customers raises issues of technology transfer and dual-use technologies.

\section{E. Policy Recommendations to Enable the Paradigm Shift}

The different barriers that may hinder the shift from today's paradigm to the fractionated spacecraft paradigm require the exploration of potential policy options that could enable such a transition. This chapter addresses these barriers and suggests recommendations to make the transition possible. 


\section{E.1 Ability and motivation to transition of the various players}

\section{E.1.1 The motivation/ability framework}

The motivation/ability framework developed by Christensen gives the possibility to map the motivation and ability of the players to implement fractionated architectures and the different barriers to the paradigm shift, in order to see if the context if favorable to fractionation. This framework was initially developed to understand the barriers to innovation in different industries and the government's role in influencing the creation and exploitation of innovation (Christensen, 2004). As presented on Figure 84, the situation of a potential innovator can be defined along two dimensions: its motivation and its ability to innovate. Its motivation mainly depends on the potential benefits it can derive from the innovation (market, competitive environment, etc.) and its ability on its resources availability and adequacy of the resources, access to the market, etc.).

In addition, the different barriers can be associated with one of these dimensions of this framework. The actions of any non-market player, like the government, can then be assessed in terms of their impact on the players' ability and motivation.

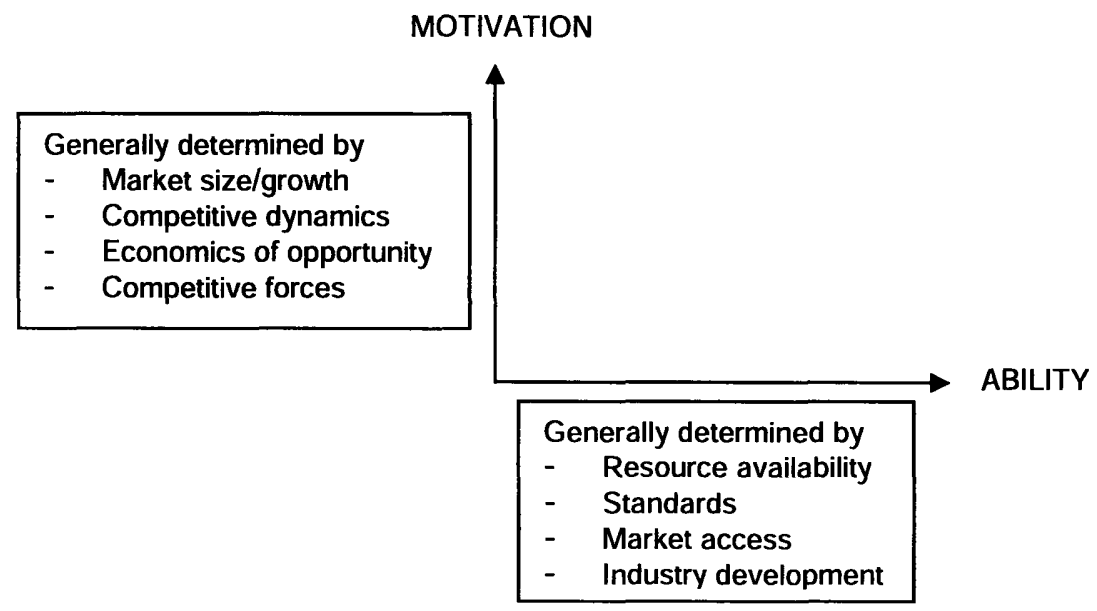

Figure 84: The motivation/ability framework

E.1.2 The motivation and ability to innovate of the different players and the barriers to the paradigm shift

For the fractionated architectures to be implemented, the current players should have both the motivation and the ability to transition towards the paradigm. However, all the barriers mentioned above sets limits to both their motivation and ability, which are clearly low. This situation is presented on Figure 85 . The motivation of the subcontractors is clearly higher than the one of the system integrators but their ability is lower. Any intervention of the government should aim at increasing the motivation and/or the ability of all the players at least up to a minimum level that would make the transition possible. These levels are defined on Figure 85 by the ability and motivation thresholds. The main barriers to the players' motivation are the small size of the market and the competitive environment. The main barriers to their ability are the lack of resources for such a risky enterprise and the total absence of standards for the interfaces. 


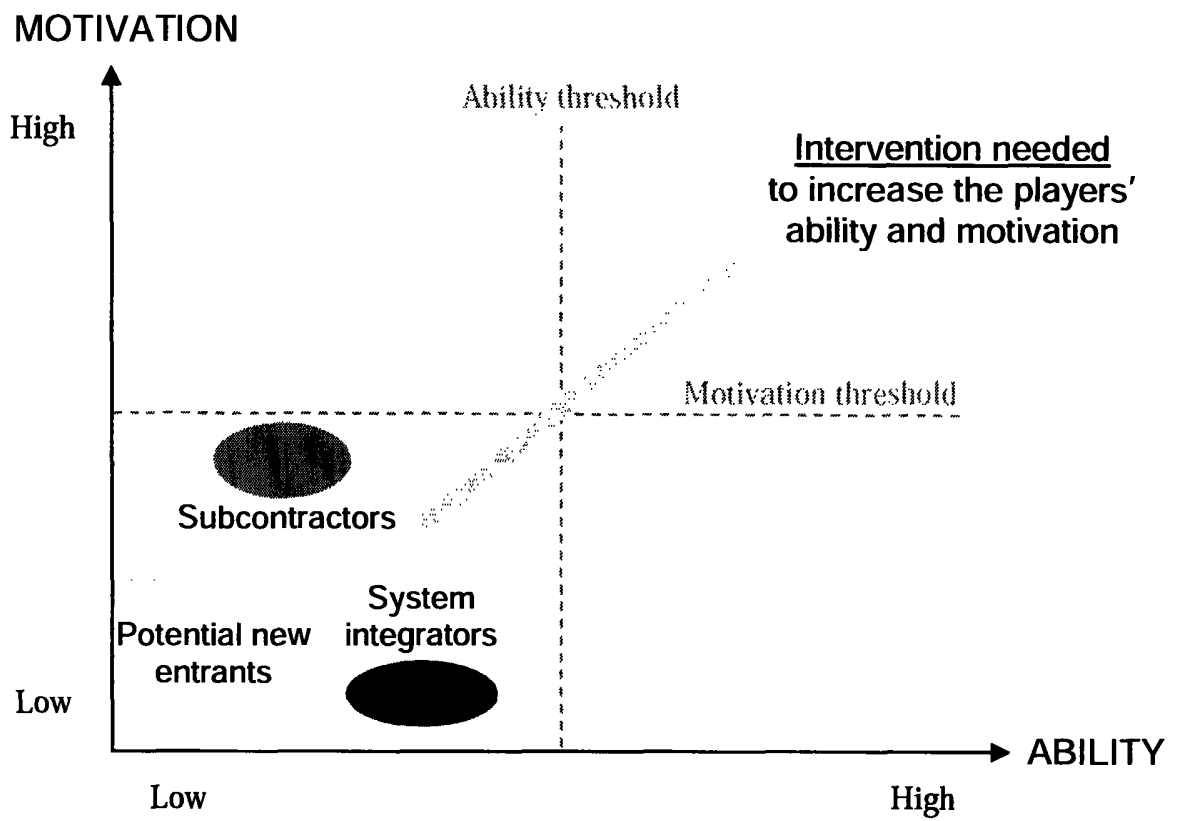

Figure 85: The motivation and ability to implement fractionation of the current industry players

\section{E.2 Rationale for government intervention}

There are various justifications for the government intervention to enable the paradigm shift. First of all, the government would be at least initially the main customer for this infrastructure which would serve interests like national security. Then, the government may intervene because of different markets failures. First, the private sector underinvests in the R\&D necessary to develop the technologies that would make fractionated spacecraft technically feasible and viable. Second, there is a need for coordination of the R\&D efforts to both develop these technologies and develop the fractionated spacecraft architectures, and the government has a role to play in bridging institutions. Third, the private sector is unlikely to take the lead in the management of the infrastructure. Another argument that supports government intervention is the lack of standards for the modules interfaces. Finally, the government intervention could be justified in order to ensure access to the infrastructure for commercial customers at a marginal cost.

\section{E.3 Market(s) for which an infrastructure could be developed}

\section{E.3.1 Requirements on the missions supported by a common infrastructure}

The architectural analysis in Part I suggests that the "best" fractionated spacecraft architecture from a customer point-of-view depends on the application (e.g., imagery, navigation, or communications). Moreover different applications usually correspond to different orbits. Therefore, in an optimal case, the infrastructure would be initially developed for a given field of application in one - or one type of - orbit, and for missions with rather similar requirements for their supporting functions. Constellations of navigation satellites or communications satellites could be a good starting point. 
E.3.2 Market to be targeted for the development of an infrastructure

The infrastructure should therefore first address a market of satellites in the same field of application and in a same orbit. Then, this market should represent a volume of satellites large enough to justify the mass-production approach of the standardized modules. Finally, if the infrastructure is to be opened later on to the commercial customers, this market should have both military and commercial customers.

Figure 86 shows the number of active satellites on-orbit in November $2005^{2}$. These numbers cover the US military satellites and the multinational satellites in which the US are involved (most of which are communications satellites). Clearly the market of communications satellites should be targeted first, as it is the market with the largest number of active satellites. Another reason to focus on this market is that communications satellites tend to be quite similar to each other. Most of them are built on the same bus platforms. Besides, the majority of the military communications satellites, and almost half of the commercial ones, are in geosynchronous orbit, therefore an infrastructure in GEO that would support communications satellites should be the first one to be developed. However communications satellites are also very power-demanding, so that the technological challenge of the power subsystems mass would really need to be addressed.

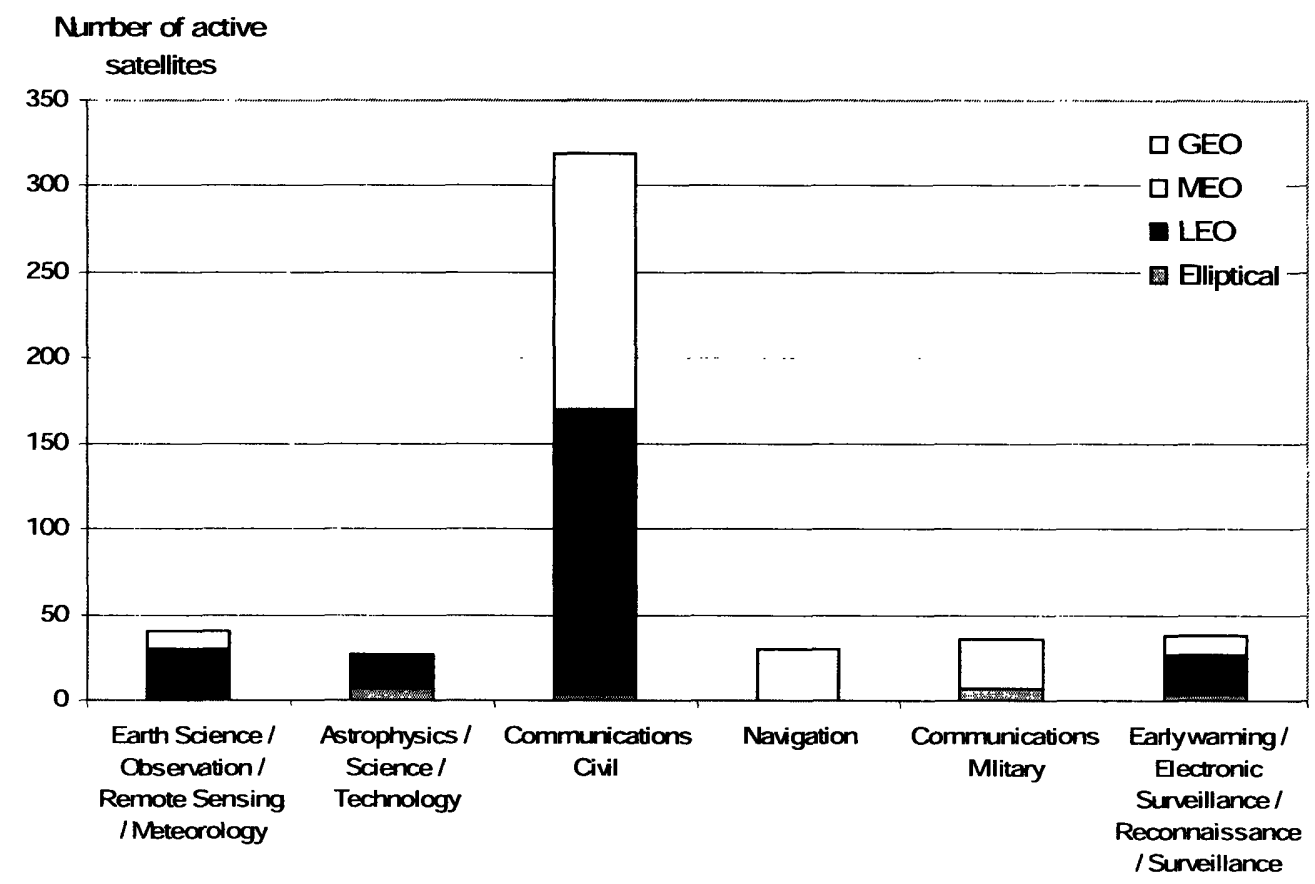

Figure 86: Active US and multinational satellites (Nov. 2005)

\footnotetext{
${ }^{2}$ Source: UCS Satellite Database 11-15-2005

http://www.ucsusa.org/global_security/space_weapons/satellite_database.html
} 


\section{E.4 Policy Recommendations}

\section{E.4.1 The US government as an "anchor tenant"}

The first policy the government should adopt is a commitment to act as an "anchor tenant" for use of this infrastructure.

The military community is expected to be the first infrastructure customer, so that the government would have a major role to play in the development and management of the infrastructure and would initially drive the demand for such an infrastructure. The government would have to be clear on the market it plans to target with this infrastructure to reduce the uncertainties that may hold the industry players back. This demand would guarantee a market, as well as the initial funding of the infrastructure development and the stability of the funding for the infrastructure management. Furthermore, this policy would attract commercial users once the infrastructure is developed. As a result, this policy would increase the motivation of all industry players.

\section{E.4.2 Investing in enabling technologies}

The second recommendation is the award by the government of $R \& D$ contracts to develop the enabling technologies.

It addresses the issue of underinvestment in the R\&D necessary for the transition towards fractionated architectures. A major barrier to this transition is actually the lack of funding for the research and development efforts necessary to develop the technologies that would make fractionated spacecraft technically feasible and viable. Even if the government being an "anchor tenant" guarantees a market, the private sector may still underinvests in the development of the enabling technologies, because of their high costs and risks. The high costs and risks associated with these technologies limit their ability to innovate. The government which is the first customer of the infrastructure has mainly two possibilities to remedy this market failure: it could either use direct subsidies or R\&D tax credits. For such specific needs, direct subsidies via R\&D contracts seem to be the best solution to support innovation at least in the early stages when risks are still high.

\section{E.4.3 Coordinating efforts}

The government should coordinate the efforts to develop the technologies and define the architecture. It would have a role to play in bridging institutions in different ways.

\section{E.4.3.1 Coordinating efforts to develop enabling technologies}

The third recommendation is the award of $R \& D$ contracts to different public-private partnerships in the early stages of the enabling technologies development.

As recommended above, the $R \& D$ efforts necessary to develop the enabling technologies would be supported by the government via R\&D contracts, at least in the early stages. Usually, competitive bidding is the procurement approach used by the government in order to increase efficiency and decrease costs. However, the firm, or firms, that would 
be awarded these R\&D contracts would gain a significant technological advantage over competitors. To avoid conflict, the government could either award different contracts to several competitors or centralize the efforts in a government laboratory or an industrial technology alliance (Chan, 2003). Centralizing efforts would be justified by efficiency concerns, but the concentration of the industry and security reasons, i.e. the need for several suppliers for these critical technologies, would rather suggest awarding several parallel contracts. Moreover, these various $R \& D$ efforts could be undertaken in two different ways: the R\&D could be done by the private sector only or within public-private partnerships. Public-private partnerships involve cooperative R\&D or co-development among industry, universities and government laboratories, and tend to facilitate technology transfer from the laboratories to the market. So the best option for the development of the enabling technologies is several parallel contracts and the development of public-private partnerships.

In general an initial public investment in high-risk technologies can enable private firms to later take advantage of the commercial possibilities of the proven technologies. Figure 87 presents the usual public-private relationship on the S-curve of emerging technologies. In a first stage, the ferment stage, the government subsidizes the research activities to develop and demonstrate new technologies. In a second stage, the take-off stage, the private sector is expected to take over and invest in applied research to bring these technologies to maturity and develop new applications. This take-off stage is made possible mostly because private firms can appropriate the technology. Therefore if the government wants the private sector to take over the development of the technologies, it would have to guarantee the private firms that they would be able to appropriate these technologies with patents. The appropriability of these technologies should not be a significant problem on the market, as there would be different suppliers for each technology thanks to the parallel R\&D contracts.

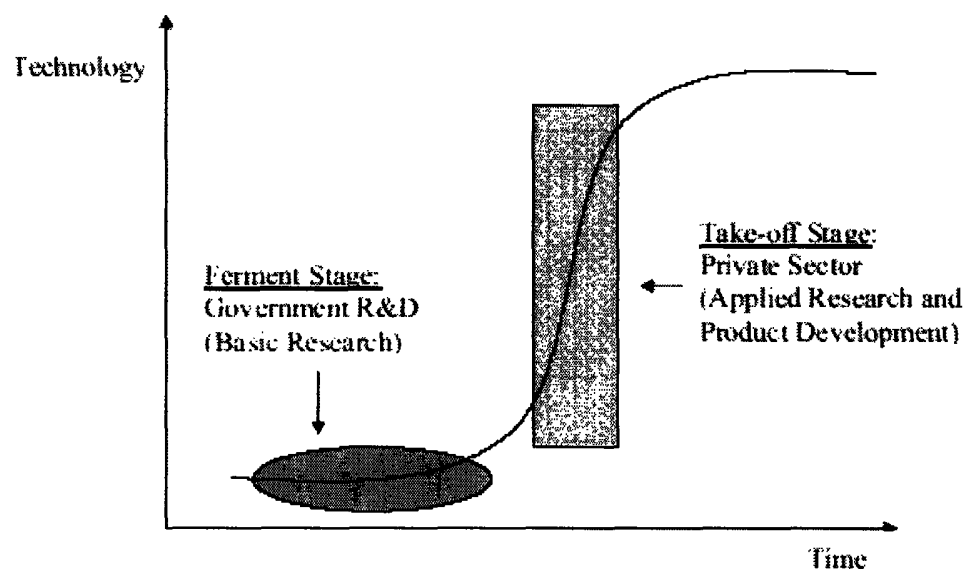

Figure 87: Public-private relationship on emerging technology S-curve (Chan, 2003) 


\section{E.4.3.2 Coordinating efforts to develop the initial fractionated spacecraft architecture}

The fourth specific recommendation is a relaxation of competition policy in order to allow a cooperative development of the fractionated spacecraft architecture.

As mentioned above, today's system integrators are in the best position to develop the fractionated spacecraft architecture because of their expertise and experience. But this architecture they would develop would bring changes in the industrial organization that they may not perceive as favorable to them. Moreover, as a maximum interoperability of the infrastructure systems is highly desirable, the chosen architecture is unlikely to be proprietary, which means a limited appropriability of this architecture for the system integrators. There is therefore little incentive for system integrators to develop this architecture in a competitive market environment. This problem can be addressed by the government with the creation of a cooperative environment. The best solution would be an industrial joint venture formed by the main system integrators, but it is prevented by anti-trust legislation and competition policies as it would put them in a monopoly situation. The recommendation is therefore to adopt a competition policy that permits cooperation for the development of the infrastructure development (Martin, 1999).

\section{E.4.4 Defining standards}

The fifth recommendation is that the government should mandate the definition of the interfaces standards.

This standards definition could be done by a standard committee. Such a committee could play a role similar to the Consultative Committee for Space Data Standards (CCSDS), which synthesizes consensus, standardized solutions for common space data handling needs (Finkleman, 2005). A large part of the fractionated spacecraft architecture definition is the definition of the standardized interfaces. There are three ways in which interface standards can be set. First of all, a standards committee can decide on the standards to be adopted and firms then take them as input in their design and manufacturing processes. Second, a standards war can take place between private firms that propose different standards, and market mechanisms lead to the adoption of a set of standards that will dominate the market. Finally, new interface standards can be only "extensions" of the existing architecture standards (Cebon, 2002). The adoption of open standards, i.e. that are publicly available, is usually considered as desirable to maximize the interoperability of the infrastructure elements. However, in the case of the fractionated spacecraft, technologies are not mature enough to rely on existing standards, except perhaps for wireless communications (Chan, 2003). Thus the Government has a role to play in defining, at least partly, the interfaces standards. These would be therefore set by a standard committee in conjunction with the laboratories and/or firms that would develop the interfaces technologies. It would be unlikely that given the risks and costs associated with the development of the infrastructure and the small size of the market, companies would fight to impose its standards. The definition of these interfaces standards by a committee would limit the uncertainties that firms would face and "level the playing field" (West, 2004). This would have a significant impact on the competition 
between private firms and would increase their motivation. These standards would allow today's subcontractors to become module suppliers, and potential new entrants to enter the market.

\section{E.4.5 Managing the infrastructure}

The sixth recommendation is that the government creates a centralized entity that will manage the infrastructure.

The long-term sustainability of the infrastructure utilization depends on the stability of the funding but also of the management of the infrastructure. The guarantee of a stable management is likely to increase the players' motivation. This entity could be public or private but is likely to be public as this infrastructure will have as first customers the military community. All or part of the infrastructure management could later be privatized. This centralized organization would include the entity responsible for the procurement of the standardized modules.

\section{E.4.6 Ensuring access to commercial users at a marginal cost}

The seventh recommendation is that the government should ensure access of commercial customers to the infrastructure at a marginal cost and take it into account in the initial development of the infrastructure.

The prospective of future commercial users, i.e. of an expansion of the market, would increase all the players' motivation. The transition from an infrastructure used only by military customers to one that may be used also by non-military customers raises issues of security and technology transfers. A good example of space-based infrastructure that originated in the military and was then used by other users is the Global Positioning System (GPS) (Chan, 2003).

If the government decides to offer to commercial users the access to the infrastructure, it would have two possibilities, either a "dual-benefit" or a "dual-use" approach. First, commercial users could use the same infrastructure as the military users (e.g., GPS) or the infrastructure could be duplicated so that military and commercial users would use similar but separate infrastructures (Chan, 2003). When considering the possibility for commercial customers to access to the existing infrastructure, the following questions should be examined (Chan, 2003): how would the infrastructure access be prioritized?; how would the infrastructure capacity be allocated?; how would military standards for security and robustness be affected?; how would the architecture of the infrastructure affected if it has to accommodate non-military users?; and how much would the commercial customers be charged to access the infrastructure?

When looking at these questions, military customers may be consider the open access to the same infrastructure as too risky because of the limited capacity and security concerns, and may prefer the option of transferring the technology and allow a commercial development of a similar infrastructure. But if the same infrastructure is used for both users, the government could charge only a marginal cost to commercial customers which would reduce the commercial applications cost and make the on-orbit infrastructure viable. A major justification for the access to the same infrastructure is that commercial 
customers could provide the military community with additional support to get funding for their common infrastructure. Obviously a trade-off between constraints and opportunities would have to be made, but on should keep in mind that a common infrastructure would serve both national security and economic interests. The constraints brought by commercial customers can be limited, if their needs are taken into account early in the design of the infrastructure.

\section{E.5 Impact of these recommended policies on the players' motivation and ability}

As explained earlier, a minimum level of motivation and ability should be reached by the different industry players to make the transition towards a fractionated spacecraft paradigm possible. All these policy recommendations improve the situation and can be classified along the two dimensions of the motivation/ability framework. Error! Reference source not found. represents the specific recommendations and their chronological sequence, from the first (1) to the last one (5) to be implemented. First, there are policies that would affect the players' motivation: those related to the size of the market, i.e. the government as an "anchor tenant" and the access to the infrastructure for commercial customers, and those related to the competitive environment, i.e. the coordination of the efforts for the developments of the enabling technologies and of the architecture. Second, there are recommendations that would affect the players' ability: the investment in R\&D, the definition of standards and the management of the infrastructure by a central entity.

This framework shows that even though all these recommendations need to be implemented, their "amplitude" can be modulated with respect to each other. Along a given dimension, these different policies can actually be traded against each other. For example, a larger market could "compensate" for a more competitive environment.

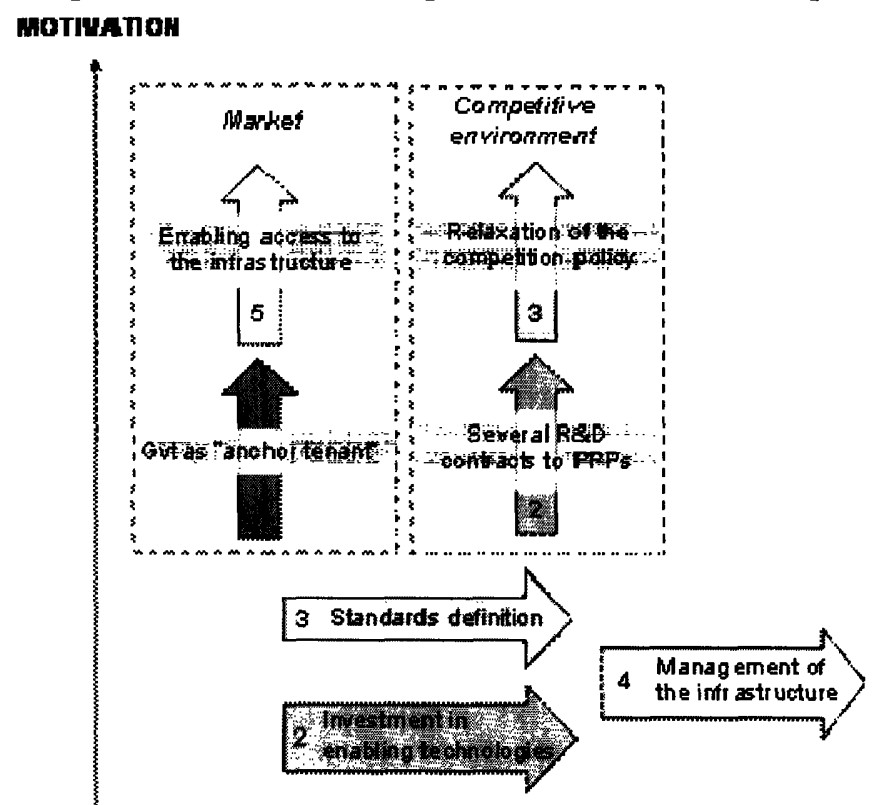


Figure 88: Impact of the recommended policies on the players' ability and motivation to implement fractionation

\section{F. Conclusions of the Industrial and Policy Analysis}

\section{F.1 Main conclusions of the industrial analysis}

The second part of this thesis investigates the reasons why, despite so many potential benefits for customers, fractionated architectures are not implemented yet and will probably not be implemented without any intervention from outside the industry. There are clearly too many financial, managerial and regulatory barriers that limit the various industry players' ability and motivation to implement fractionation. First of all, fractionation creates a whole new industrial paradigm in which the traditional protoflight approach take aspects of a mass production approach. This change in industrial paradigm could benefit customers. However, it would affect the space industry organization so that today's system integrators are likely to resist this change. Second, there are significant barriers associated with the development of the enabling technologies and with the development and management of the fractionated modules infrastructure. There are several rationales for a government intervention that could enable the paradigm shift. First, the government would be the first customer of the infrastructure. Second, only the government can correct some of the existing market failures that prevent the implementation of fractionation. Last but not least, only the government can ensure the access of commercial customers to the infrastructure at marginal cost. The various policy options that may lower these barriers are explored and the question of the initial market for fractionated spacecraft is addressed. The communications satellites market was identified as the first market to target; the infrastructure could be initially developed to support military communications missions and then its access may be opened to commercial communications payloads. Finally, a unique set of policies that would make the paradigm shift possible is proposed. This recommended chronological sequence of policies is the one that follows.

1) The government should commit to act as an "anchor tenant" for use of the infrastructure;

2) The government should invest in the enabling technologies;

3) The government should award R\&D contracts to different public-private partnerships to develop the enabling technologies, at least in the early stages;

4) Competition policy should be relaxed in order to allow a cooperative development of the fractionated spacecraft architecture;

5) The government should mandate the definition of the interfaces standards;

6) The government should create a centralized entity that would manage the infrastructure.

The shift from today's traditional paradigm to the new fractionated spacecraft one might occur only if all of these policies are implemented. This list shows the challenge that this transition represents. 


\section{F.2 Innovation in the space market}

Fractionation illustrates the difficulty for innovation to take place in the space market, at least at architecture level. In the conventional innovation model, innovation often comes from outsiders (Utterback, 1994) but the space systems market has barriers to entry that are today too high for such a scenario to occur. Any architectural innovation has then to come from within the current industry and as it would certainly require changes in the current "rules of the game" this type of innovation faces many barriers. Thus the structure of today's space industry is very stable. This structure and the current industrial paradigm are strongly connected to the dominant spacecraft architecture. This architecture was inherited from a time when the performance of space systems, defined in traditional terms, was much more important than their flexibility and responsiveness. However, needs of some of the space systems customers have evolved since that time, and for some military and commercial customers, these non-traditional attributes have become today more important. These needs are unlikely to be satisfied by traditional spacecraft, but as demonstrated in this thesis the adoption of new architectures that may better fulfill these needs and the shift to a corresponding new industrial paradigm are very difficult.

\section{F.3 The need for a new organizational structure}

If fractionated spacecraft are implemented, not only the organization of the space industry would have to change but also the structure of the different governmental entities involved in the different lifecycle phases of space systems. An architectural innovation like fractionation would call for a new organization of the entities that develop and procure space systems and for the creation of new ones, at least for the management of the infrastructure. It has been demonstrated that the architecture of a system and the structure of the organizations responsible for them are strongly connected (Garud and al., 2002). Thus the organizations structure would have to adapt to the new fractionated architecture. Fractionated spacecraft consist of two different types of elements: the payload modules and the infrastructure modules. These elements are likely to require separate entities that would have to be added to the existing ones that are responsible for traditional spacecraft. The new structure of these organizations and their responsibilities and tasks should be the subject of future research. 


\section{CONCLUSIONS}

\section{A. Main conclusions of the assessment of the fractionated spacecraft concept}

The first conclusion of this assessment is that fractionated spacecraft could deliver more value to customers than traditional ones for a given mission and at a given level of performance. As demonstrated in the architectural analysis, the more fractionated the architecture is, the more expensive it is. But in terms of value delivered to potential customers the "best" fractionated architectures are different in the various fields of application (communications, navigation, etc.). According to the examples presented in Part I, for communications missions, the more fractionated the architecture is, the more utility it could deliver to customers, whereas for navigation missions utility tend to decrease with fractionation. These differences are linked to the technical characteristics of the spacecraft as well as to the difference in the needs of the users' communities. The utility interviews have shown that users in different fields of application show different interest in the various forms of flexibility. A second important conclusion of the architectural analysis is that, if the infrastructure is developed and for a same level of performance, some of the fractioned architectures may dominate traditional ones both in terms of cost and utility.

This thesis has clearly demonstrated that fractionation could provide customers with many potential benefits, but it has also shown that this concept is unlikely to be implemented without external intervention, as the private sector has little incentive to implement it. This study showed that fractionation could create a whole new paradigm and presented the new space industry organization that would correspond to this paradigm. It also demonstrated that the various industry players have neither the motivation nor the ability to make the shift from today's paradigm to the new fractionated spacecraft paradigm possible. The government, as such and as the first customer of the infrastructure, is in the best position to enable this transition. Thus, this thesis makes specific policy recommendations that address each of the barriers to the industry players' motivation and ability and that could make the paradigm shift possible.

\section{B. Main contributions of the thesis}

This research, like any other, raised many questions that are not directly related to the fractionated spacecraft concept. Some of these challenges led to analyses and to the development of methods that are relevant and could be used for other research topics. This section summarizes the main "indirect" contributions of this thesis.

A first contribution of this thesis is the framework developed to analyze and define the different forms of flexibility. As both users' interviews confirmed, flexibility means many things to different people and there are many words that are associated with it. This simple framework can be reused for other complex systems analysis by adapting the 
different dimensions. It can surely be further improved but it represents a good starting point for any discussion on what flexibility and its different forms are.

A second contribution of this thesis is the use of non-traditional performance parameters in a Multi-Attribute Tradespace Exploration (MATE). So far systems architectures had been compared in a MATE approach in terms of traditional performance parameters, but this study compared fractionated and traditional architecture at a same level of performance - as traditionally defined - in terms of non-traditional performance attributes. This framework with such attributes can be used for investigating different architectures of other complex systems, with non-traditional attributes only or with both traditional and non-traditional attributes. However the choice of non-traditional attributes such as the ones chosen in this study clearly makes the analysis even more complex than with traditional performance attributes, as these attributes are context- and systemspecific. It therefore requires defining for each mission specific scenarios that best represent the different forms of flexibility.

A third contribution of this thesis is the use utility interviews with non-traditional performance parameters. The three-step process developed for this study could be used for other studies. The questionnaire can be easily adapted to other systems. Moreover, the visuals designed for these interviews could be used for similar interviews whatever the attributes and the number of attributes are.

\section{Future research}

\section{C.1 Further analysis of the fractionated architectures}

C.1.1 Taking more scenarios into account in the cost/utility analysis

The architectural analysis was done with a limited set of attributes. This number of attributes could be extended or modified to include other attributes. For instance, traditional and non-traditional performance attributes could be combined if more data on the performance of fractionated architectures become available. However, one should keep in mind that the total number of attributes is limited by the utility interviews. One way to include more attributes without the constraints of the interviews on the number of attributes is to consider for a single attributes different scenarios with the same consequences at system level. A single utility function corresponding to the attribute of maintainability could be used to analyze the failure of different subsystems.

\section{C.1.2 Investigating other fractionated architectures}

Despite the apparent complexity of the architectural analysis, only a limited number of fractionated architectures were actually investigated in this part of the thesis. A specific technology sequence has been chosen based on today's maturity of some of the enabling technologies and some of the architectures were not taken into account in the architectural matrix. Thus the architectural analysis covers what can be considered today as the most relevant fractionated architectures, but clearly not all the possible architectures. Some fractionated architectures that are not relevant today may become relevant in the future if new technologies are developed or new needs identified. The analysis of other fractionated architectures could therefore be made. 
The potential synergies between homogenous and heterogeneous fractionation have been mentioned in this thesis. Architectures that would combine these two concepts could also be investigated in this framework and compared in terms of utility and cost with traditional and heterogeneously fractionated ones. These architectures would consist of different modules, but some of the supporting functions would be provided by several identical modules. For instance, instead of a single large power module the spacecraft would have several smaller power modules.

\section{C.1.3 Improving the mass, cost and attributes figures}

As mentioned in the architectural analysis, the analyses are based on traditional mass and cost models that may not be favorable to fractionated architectures. If more mass and cost data become available, the analyses could be improved. Besides, the time model used was quite simple so the time results can be improved if models for development, manufacturing, and integration and testing times of the spacecraft and its different subsystems become available. The analysis could be further improved as the enabling technologies are developed and brought to maturity and more data on their mass, cost and performance become available.

\section{C.1.4 Doing additional utility measurement}

For this thesis, two utility interviews have been realized in two different fields of application: communications and navigation. More interviews could be done in these same fields to better capture space systems users needs and preferences. Interviews could also be done in other fields of application. Finally, as the interviews done for this thesis confirmed, in a same field of application different users communities may also have different need and preferences. Thus in the various fields of application, groups of users should be defined and interviewed.

\section{C.2 Further research on fractionation}

\section{C.2.1 Potential benefits and risks of fractionation}

Further research on the fractionated spacecraft concept could complement this study. Detailed analyses had already been done on all the missions that distributed architectures make possible, and similar analyses could be done for fractionated architectures. Besides, the potential synergies of fractionation with concept like on-orbit servicing that would enable even more sustainable space utilization should be examined.

The risks associated with fractionation have been mentioned in this thesis but further work can be done on them. The technical risks should obviously be studied if more information on the enabling technologies becomes available. The impact of fractionation on space debris is also a topic that could be further investigated.

\section{C.2.2 Changes in the organizations structure}

A last challenge mentioned in the conclusion of the industrial analysis and which should be further investigated is the adaptation of today's organizations to this new space systems architecture. 


\section{BIBLIOGRAPHY}

Allen, T., Moses, J., Hastings, D., Lloyd, S., Little, J., McGowan, D., Magee, C., Moavenzadeh, F., Nightingale, D. Roos, D., and Whitney, D., "ESD Terms and Definitions" Version 12, Working Paper Series, Engineering Systems Division, Oct. 2001

Baldwin, C., and Clark, K., "Design Rules: The Power of Modularity", MIT Press, 2000

Baldwin, C., and Clark, K., "Modularity in the Design of Complex Engineering Systems", January 2004

Brown, O., Eremenko, P., and Roberts, C., "Cost-Benefit Analysis of a Notional Fractionated Space Architecture", Space 2005, Long Beach, California, Aug. 30-1, 2005

Cebon, P., Hauptman, O., Shekhar, C., "Industries in the Making: Product Modularity, Technological Innovation and the Product Lifecycle", June 2002

Chan, S., "Investing in the Development of a Space-Based Information Network Infrastructure", PhD Thesis, Engineering Systems Division, MIT, August 31, 2003

Chen, B., and Shi, Y., "Generating and Appropriating Value: The Case of Aerospace Systems Integrators", Centre for International Manufacturing, Cambridge University, UK, The 6th International Symposium on International Manufacturing, Cambridge, UK, September 2001

Christensen, C., "The Innovator's Dilemma, When New Technologies Cause Great Firms to Fail", Harvard Business School Press, 1997

Christensen, C., Roth, E., and Anthony, S., "Seeing What's Next: Using Theories of Innovation to Predict Industry Change", Harvard Business School Press, May 2004

Christian, J., and Olds, J.,“A Quantitative Methodology for Identifying Evolvable Space Systems", AIAA 2005-2543, 1st Space Exploration Conference: Continuing the Voyage of Discovery, 30 Jan. - 1 Feb. 2005, Orlando

De Neufville, R., Scholtes, S., and Wang, T., "Valuing Options by Spreadsheet: Parking Garage Case Example”, ASCE Journal of Infrastructure Systems, in press, 2005

De Neufville, R., "Applied Systems Analysis: Engineering Planning and Technology Management", McGraw-Hill, Inc; New York, New York, USA ISBN: 0-07-016372-3 1990

De Weck, O., de Neufville R. and Chaize M., "Staged Deployment of Communications Satellite Constellations in Low Earth Orbit", Journal of Aerospace Computing, Information, and Communication, 1, 119-136, March 2004 
De Weck, O., de Neufville, R., and Chaize M., "Enhancing the Economics of Communication Satellites via Staged Deployment and Orbital Reconfiguration", AIAA2003-6317, AIAA Space 2003 Conference and Exhibition, Long Beach, California, September 2003

Dosi, G., "Sources, Procedures, and Microeconomic Effects of Innovation", Journal of Economic Literature, Volume 26, September 1988, pp. 1120-71

Finkleman, D., "Space Standards, Rules, Innovation, and Inhibition", AAS 05-198, AAS/AIAA Space Flight Mechanics Conference, Copper Mountain, Colorado, January 23-27, 2005

Frigant, V., "Modularity: the Foundations of an Architect Firm", Cahiers du GRES, Cahier 2004-02, January 2004

Garud , R., Arun Kumaraswamy, A., and Langlois, R., eds, "Managing in the Modular Age: Architectures, Networks, and Organizations", Blackwell Publishers, September 2002)

Hassan, R., de Neufville, R.. de Weck, O., Hastings, D., and McKinnon, D., "Value-atRisk Analysis for Real Options in Complex Engineered Systems", IEEE Conference on Large Scale Infrastructures, Hawaii, October 2005

Hastings, D., Lamassoure, E., Weigel, A., and Saleh, J., "Policy Enablers for the Development of a Space-Based Infrastructure", The $5^{\text {th }}$ International Conference on Technology, Policy and Innovation, Delft, The Netherlands, June 2001

Hastings, D., and McManus, H., "A Framework for Understanding Uncertainty and its Mitigation and Exploitation in Complex Systems", 2005 INCOSE International Symposium, Rochester, New York, July 2005

Hobday, M., “Complex System vs Mass Production Industries: A New Innovation Research Agenda", Paper prepared for CENTRIM/SPRU/OU Project on Complex Product Systems, EPSRC Technology Management Initiative, GR/K/31756, June 1996

Keeney, R., and Raiffa, H., "Decisions with Multiple Objectives: Preferences and Value Trade-offs", John Wiley \& Sons, New York, NY, 1976

Martin, S., "The Nature of Innovation Market Failure and the Design of Public Support for Private Innovation", Centre for Industrial Economics, University of Copenhagen, March 1999

McManus, H., Hastings, D., and Warmkessel, J., "New methods for Rapid Architecture Selection and Conceptual Design", Jan. 2004 
Nilchiani, R., "Measuring the Value of Space Systems Flexibility: A Comprehensive Sixelement Framework", PhD Thesis, MIT, 2005

Nilchiani, R., and Hastings, D., "Defining Software Flexibility in Space Systems", Space 2004 Conference, San Diego, California, Sept. 2004

Nilchiani, R., Hastings, D., Joppin, C., "Calculations of Flexibility in Space Systems", Engineering Systems Symposium Proceedings, MIT, March 2004

Ross, A., "Multi-attribute Tradespace Exploration with Concurrent Design as a Valuecentric Framework for Space System Architecture and Design", Master's Thesis, MIT, 2003

Roth, R., Field, F., and Clark, J., "Materials selection and multi-attribute utility analysis", Journal of Computer-Aided Materials Design, V 1 No 3, ESCOM Science Publishers, Leiden, The Netherlands, October, 1994

Sarsfield, L., "The Cosmos on a Shoestring, Small Spacecraft for Space and Earth Science", RAND, Monograph Reports, MR-864-OSTP, 1998

Spaulding, T., "Tools for Evolutionary Acquisition: a Study of Multi-Attribute Tradespace Exploration (MATE) applied to the Space Based Radar (SBR)", Master's Thesis, MIT, 2003

Tushman, M., and Anderson, P., "Managing Strategic Innovation and Change: A Collection of Readings", Oxford University Press, USA, August 1, 1996

Utterback, J., "Mastering the Dynamics of Innovation", Harvard Business School Press, September 1996

Von Neumann, J., and Morgenstern, O., "Theory of Games and Economic Behavior", 2nd edition, Princeton University Press, Princeton NJ, 1947

Wertz, J., and Larson, W., "Space Mission Analysis and Design”, 3rd Edition, Space Technology Library, Springer, Sept. 1999

West, J., "What are Open Standards? Implications for Adoption, Competition and Policy", Standards and Public Policy Conference, Federal Reserve Bank of Chicago, Chicago, Illinois, May 13, 2004 


\section{Appendix A - Preliminary Questionnaire}

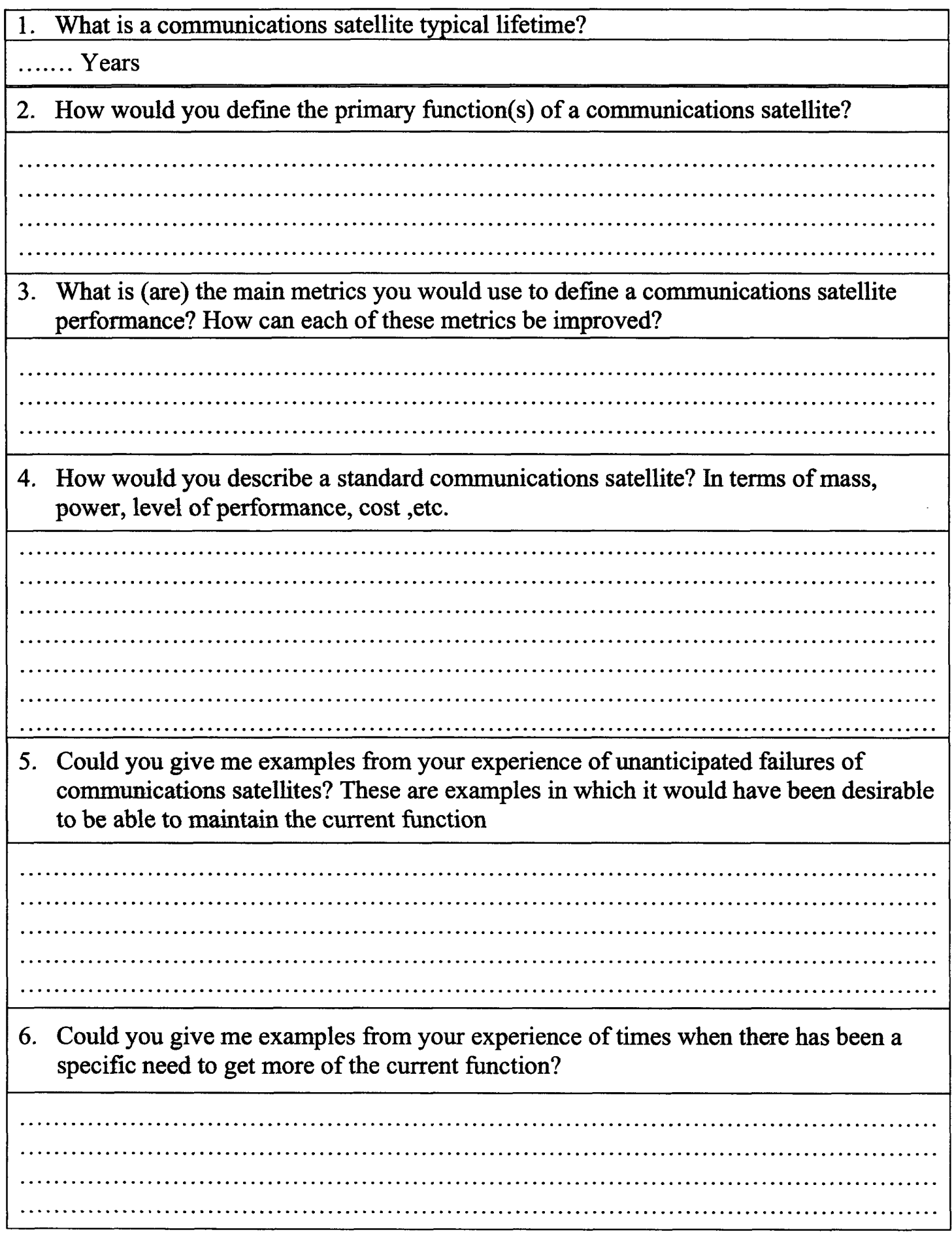


7. What function(s), different from the current one(s), do you think may be needed in the future from a communications satellite?

8. What type of change(s) is (are) most likely to occur during a communications satellite lifetime? (Multiple answers possible)

Failure - Need to maintain the current function

$\square$ Need to get more of the current function

․ Need to get a different function

9. What type of change(s) is (are) least likely to occur during a communications satellite lifetime? (Multiple answers possible)

$\square$ Failure - Need to maintain the current function

$\square$ Need to get more of the current function

$\square$ Need to get a different function

10. Which possibility do you consider as most valuable for a communications satellite?

$\square$ Maintaining the current function

$\square$ Getting more of the current function

$\square$ Getting a different function

11. Which possibility do you consider as least valuable for a communications satellite?

Maintaining the current function

$\square$ Getting more of the current function

$\square$ Getting a different function

12. Could you rank these 6 forms of flexibility in order of importance to you? From the most important (1) to the least important (6)?

..... Maintaining the current function cheaply

...... Maintaining the current function quickly

...... Getting more of the current function cheaply

..... Getting more of the current function quickly

...... Getting a different function cheaply

...... Getting a different function quickly

13. In case of failure, you will consider in your decision the costs of maintaining your satellite. What costs would you mainly take into account? (Multiple answers possible)

口 Manufacturing costs

- Integration, assembly, and test costs

Launch costs

- Other costs: 
14. In case of failure, you will consider in your decision the time necessary to maintain your satellite. What times would you take into account? (Multiple answers possible)

$\square \quad$ Manufacturing time

$\square \quad$ Integration, assembly, and test time

Launch time

$\square \quad$ Other time:

15. If you need more of the current function, you will consider in your decision the corresponding costs. What costs would you include? (Multiple answers possible)

$\square \quad$ Development costs

$\square \quad$ Manufacturing costs

- Integration, assembly, and test costs

$\square \quad$ Launch costs

$\square$ Other costs:

16. If you need more of the current function, you will consider in your decision the time necessary to obtain it. What times would you include? (Multiple answers possible)

$\square \quad$ Satellite development time

$\square \quad$ Satellite manufacturing time

․ Satellite integration, assembly, and test time

$\square \quad$ Satellite launch time

$\square$ Other time:

17. If you need a different function, you will consider in your decision the corresponding costs. What costs would you include? (Multiple answers possible)

$\square \quad$ Satellite development costs

- Satellite manufacturing costs

$\square$ Satellite integration, assembly, and test costs

$\square \quad$ Satellite launch costs

$\square$ Other costs:

18. If you need a different function, you will consider in your decision the time necessary to obtain it. What times would you include? (Multiple answers possible)

$\square \quad$ Satellite development time

$\square \quad$ Satellite manufacturing time

$\square \quad$ Satellite integration, assembly, and test time

$\square \quad$ Satellite launch time

$\square$ Other time:

19. How long would it typically take for such a communications satellite to be developed (from conceptual design to its delivery)? 
20. Once designed, how long would it typically take for such a communications satellite to be manufactured (from beginning of manufacturing process to its delivery)?

Years

21. Under nominal conditions, what is the likelihood of unanticipated failure of such a communications satellite?

22. Would the failure tend to be only partial or complete?

$\square$ Complete $\square$ Partial

23. When would you expect the satellite to fail? Please represent the periods during which it is most and least likely to fail or a failure profile on the timelines below.

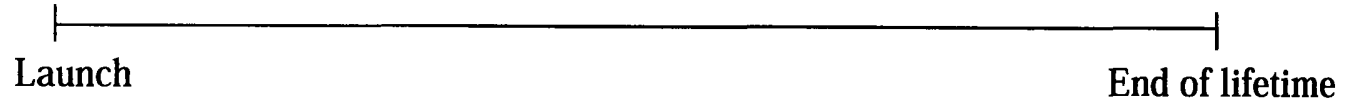

24. Which subsystems are most likely to fail? Please rank them from the most to the least likely.

..... Payload

..... Power

..... Communications

..... Control and Data Handling

..... Propulsion

..... Attitude Determination and Control

..... Thermal

25. What is the likelihood of failure of these subsystems during the communications satellite lifetime? (as a percentage or as an absolute value)

Payload..........

Power.

Communications.

Control and Data Handling..........

Propulsion..........

Attitude Determination and Control..........

Thermal......... 
26. Each of these subsystems may partially or completely fail. Will the failure of each of these subsystems more likely lead to a partial or complete failure of the whole system?

$\underline{\text { Payload }}$

$\square$ Complete $\square$ Partial ....\% of the initial capacity lost

Power

$\square$ Complete $\square$ Partial ....\% of the initial capacity lost

Communications

$\square$ Complete $\square$ Partial ....\% of the initial capacity lost

Control and Data Handling

$\square$ Complete $\square$ Partial ....\% of the initial capacity lost

Propulsion

$\square$ Complete $\square$ Partial ....\% of the initial capacity lost

Attitude Determination and Control

$\square$ Complete $\square$ Partial ....\% of the initial capacity lost

Thermal

$\square$ Complete $\square$ Partial ....\% of the initial capacity lost

27. When would you expect these subsystems to fail? Please represent the periods during which they are most and least likely to fail or a failure profile on the timelines below.

\section{Payload}

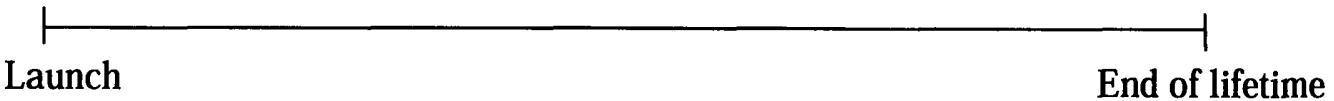

Power

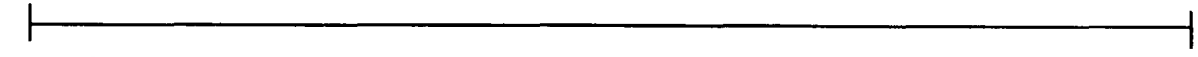

Launch

End of lifetime

Communications

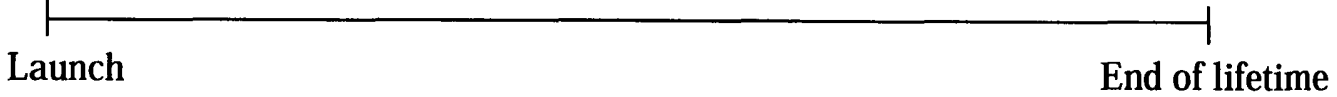

Control and Data Handling

$\begin{array}{ll}\text { Launch } & \text { End of lifetime }\end{array}$




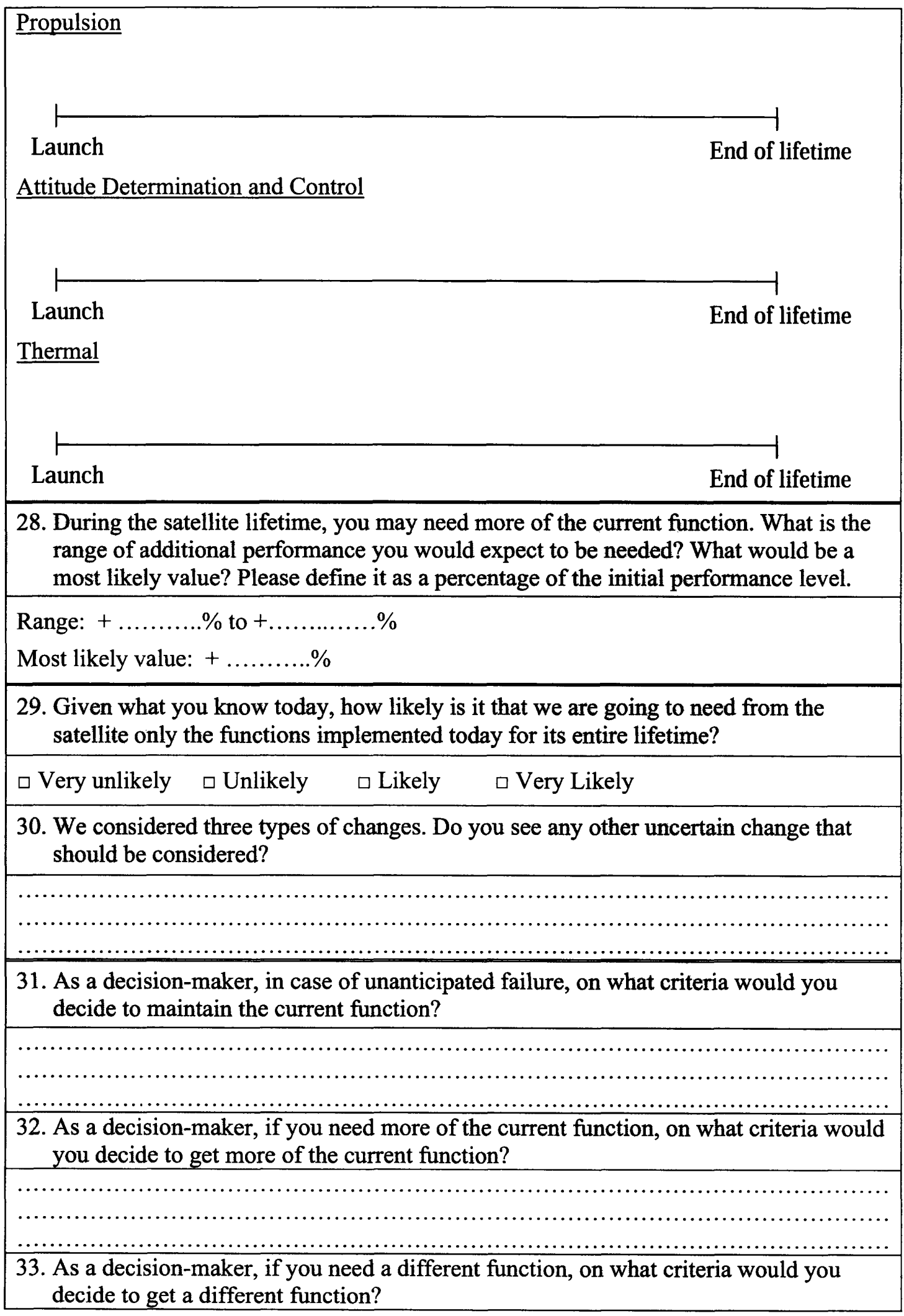


34. What would be the least acceptable cost to maintain the current function of a communications satellite in case of failure? For instance as a percentage of satellite initial cost or as an absolute value.

35. What would be the most acceptable cost to maintain the current function of a communications satellite in case of failure? For instance as a percentage of satellite initial cost or as an absolute value.

36. What would be the least acceptable cost to get more of the current function of a communications satellite? For instance as a percentage of satellite initial cost or as an absolute value.

37. What would be the most acceptable cost to get more of the current function of a communications satellite? For instance as a percentage of satellite initial cost or as an absolute value.

38. What would be the least acceptable cost to get a different function? For instance as a percentage of satellite initial cost or as an absolute value.

39. What would be the most acceptable cost to get a different function? For instance as a percentage of satellite initial cost or as an absolute value.

40. What would be the least acceptable time necessary to maintain the current function of a communications satellite in case of failure? For instance as a percentage of satellite initial time to delivery or as an absolute value.

41. What would be most acceptable time necessary to maintain the current function of a communications satellite in case of failure? For instance as a percentage of satellite initial time to delivery or as an absolute value.

42. What would be the least acceptable time necessary to get more of the current function of a communications satellite? For instance as a percentage of satellite initial time to delivery or as an absolute value. 
43. What would be the most acceptable time necessary to get more of the current function of a communications satellite? For instance as a percentage of satellite initial time to delivery or as an absolute value.

44. What would be the least acceptable time necessary to get a different function? For instance as a percentage of satellite initial time to delivery or as an absolute value.

45. What would be the most acceptable time necessary to get a different function? For instance as a percentage of satellite initial time to delivery or as an absolute value. 